\title{
Exploring and improving treatment adherence in patients with type 2 diabetes mellitus using eHealth
}

Citation for published version (APA):

Vluggen, S. (2019). Exploring and improving treatment adherence in patients with type 2 diabetes mellitus using eHealth: the development and evaluation of a web-based computer-tailored program: My Diabetes Profile. [Doctoral Thesis, Maastricht University]. https://doi.org/10.26481/dis.20191219sv

Document status and date:

Published: 01/01/2019

DOI:

10.26481/dis.20191219sv

Document Version:

Publisher's PDF, also known as Version of record

\section{Please check the document version of this publication:}

- A submitted manuscript is the version of the article upon submission and before peer-review. There can be important differences between the submitted version and the official published version of record.

People interested in the research are advised to contact the author for the final version of the publication, or visit the DOI to the publisher's website.

- The final author version and the galley proof are versions of the publication after peer review.

- The final published version features the final layout of the paper including the volume, issue and page numbers.

Link to publication

\footnotetext{
General rights rights.

- You may freely distribute the URL identifying the publication in the public portal. please follow below link for the End User Agreement:

www.umlib.nl/taverne-license

Take down policy

If you believe that this document breaches copyright please contact us at:

repository@maastrichtuniversity.nl

providing details and we will investigate your claim.
}

Copyright and moral rights for the publications made accessible in the public portal are retained by the authors and/or other copyright owners and it is a condition of accessing publications that users recognise and abide by the legal requirements associated with these

- Users may download and print one copy of any publication from the public portal for the purpose of private study or research.

- You may not further distribute the material or use it for any profit-making activity or commercial gain

If the publication is distributed under the terms of Article $25 \mathrm{fa}$ of the Dutch Copyright Act, indicated by the "Taverne" license above, 


\section{Exploring and improving treatment adherence in patients with type 2 diabetes mellitus using eHealth}

The development and evaluation of a web-based computer-tailored program: My Diabetes Profile

Stan Vluggen 
(c) copyright Stan Vluggen, Maastricht 2019

Printing: ProefschriftMaken || www.proefschriftmaken.nl

ISBN 9789463805902

All rights reserved. No part of this publication may be reproduced, stored in a retrieval system or transmitted, in any form or by any means, electronic, mechanical, photocopying, recording or otherwise, without prior permission of the author or the copyright-owning journals for previous published chapters. 


\section{Exploring and improving treatment adherence in patients with type $\mathbf{2}$ diabetes mellitus using eHealth}

The development and evaluation of a web-based computer-tailored program: My Diabetes Profile

Proefschrift

ter verkrijging van de graad van doctor aan de Universiteit Maastricht,

op gezag van de Rector Magnificus, Prof. dr. Rianne M. Letschert,

volgens het besluit van het College van Decanen,

in het openbaar te verdedigen

op donderdag 19 december 2019 om 12.00 uur

door

\section{Stan Vluggen}

Geboren op 29 juni 1989

Kerkrade 


\section{Promotors}

Prof. dr. H. de Vries

Prof. dr. N.C. Schaper

\section{Copromotors}

Dr. C. Hoving

\section{Beoordelingscommissie}

Prof. dr. N.K. de Vries (voorzitter)

Prof. dr. S.P.J. Kremers

Prof. dr. G.D.E.M. van der Weijden

Prof. dr. C.A.W. Bolman (Open Universiteit, Heerlen)

Dr. H.M. van Keulen (TNO, Leiden) 
to my dearest friend Kai Hendriks

$$
(1988-2017)
$$

If today was not an endless highway

If tonight was not a crooked trail

If tomorrow wasn't such a long time

Then lonesome would mean nothing to you at all

Yes, and only if my own true love was waitin'

Yes, and if I could hear her heart a-softly poundin'

Only if she was lyin' by me

Then l'd lie in my bed once again

(Bob Dylan, 1962) 



\section{Contents}

Chapter 1 General Introduction 9

PART 1: Formative research, program development and trial protocol

Chapter 2 Exploring beliefs on diabetes treatment adherence among

Dutch type 2 diabetes patients and healthcare providers

Chapter 3 Psychological predictors of adherence to oral hypoglycaemic agents: An application of the ProMAS questionnaire

Chapter 4 A web-based program to improve treatment adherence in patients with type 2 diabetes: Development and study protocol

PART 2: Recruitment and effectiveness evaluation

Chapter 5 Exploring factors influencing recruitment success of nurses recruiting diabetes patients for a randomized controlled trial

Chapter 6 The effectiveness of a web-based computer-tailored program to improve treatment adherence in patients with type 2 diabetes:

A randomized controlled trial

Chapter 7 General Discussion

Valorization Addendum

References

Summary 169

Samenvatting 175

Dankwoord 181

About the author 


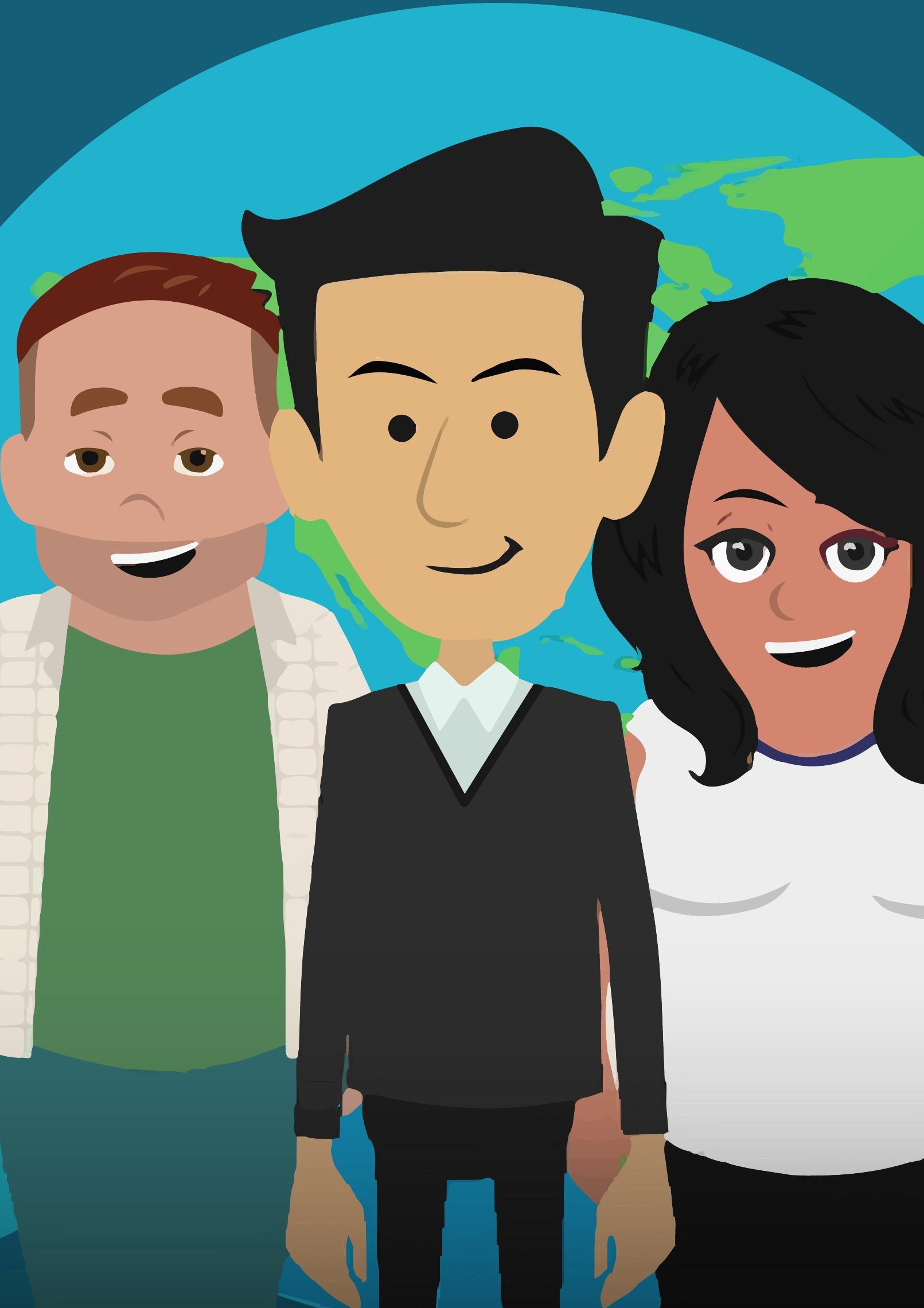




\section{Chapter 1}

General Introduction 


\section{Introduction}

Diabetes Mellitus is a chronic progressive disease characterized by hyperglycemia and the inability of the body to maintain a favorable glucose metabolism (1). Worldwide over 400 million people - and in The Netherlands over 1.1 million people - live with diabetes, and around $90 \%$ of these people have type 2 diabetes mellitus (T2DM) (1-3). The global prevalence of diabetes is projected to increase with approximately 50\% to over 600 million in 2045, and the Dutch prevalence is expected to increase a similar percentage $(1,4)$. This epidemic is largely attributable to unhealthy energy balance-related behaviors such as a lack of physical activity (PA) and unhealthy dietary patterns, a global overweight and obesity epidemic, and unmodifiable risk factors such as an increasing age and a family history of diabetes $(1,5,6)$.

T2DM is characterized by resistance to the blood glucose lowering effect of the hormone insulin in combination with $\beta$-cell dysfunction. $\beta$-cells are located in the pancreas gland and are responsible for the production, storage, and release of the insulin hormone when the blood glucose levels rise. Insulin transports glucose from the bloodstream into body cells such as muscle or fat cells where it is stored or converted into energy, which is necessary to the execution of day-to-day tasks (1). An inadequate insulin production and the inability of the body to optimally respond to insulin play a key part in the pathogenesis of T2DM and generally underlie the disrupted glucose metabolism. In T2DM, both the liver and muscles, two organs that play an essential role in glucose homeostasis, are insulin resistant which is characterized by increased glucose production by the liver and poorer glucose uptake by muscles resulting in elevated blood glucose levels, i.e. hyperglycemia (1). The most important indicator for long-term glycemic control is the level of glycosylated hemoglobin (HbA1c) which is an estimate of the average blood glucose levels over the past two to three months. An HbA1c-level of $\leq 53 \mathrm{mmol} / \mathrm{mol}(\leq 7 \%)$ is associated with a small risk of diabetic complications while this risk progressively increases with higher $\mathrm{HbA1c}$-levels. Values above $53 \mathrm{mmol} / \mathrm{mol}$ (7\%) are therefore frequently referred to as poor glycemic control but higher values are accepted in daily practice when the benefits of treatment are very limited or the risks of treatment are deemed too high (1).

\section{Consequences of poor glycemic control in type $\mathbf{2}$ diabetes mellitus}

Prolonged hyperglycemia is associated with considerable comorbidity and complications including foot ulcers, neuropathy, nephropathy, and retinopathy $(1,7)$. In addition, T2DM is one of the main causes of cardiovascular diseases (CVD) such as stroke, myocardial infarction, and peripheral arterial disease. In fact, people with diabetes are 2-3 times more likely to suffer from a CVD, compared to those who are not affected. Further, poor glycemic control is associated with an increased risk of oral health problems, enhanced rates of cancer, physical and cognitive decline, and depression (1, 8-10). Worldwide, in 2017, 4 million deaths were attributable to diabetes and its complications and total global healthcare expenditures were estimated at 727 billion United States Dollars (1). In the Netherlands, in 2017, almost 
3.000 deaths were accounted for by diabetes and its complications, and in 2015, national expenditures were estimated at 1.6 billion euros $(11,12)$. Next to the human and economic burden, diabetes can also leave its mark on society, reflected by drained healthcare budgets, frequent hospitalizations of patients, loss of productivity, and impact families of the individual affected by diabetes, e.g. through reduced spending limits for families or demanding informal caregiving (1). However, if adequate diabetes management is achieved, disease related complications can be delayed or even prevented, especially when diabetes is early diagnosed. Hence, despite the progressive nature of T2DM, patients who adhere to treatment strategies can live long and high-quality lives (1).

\section{Management of type 2 diabetes mellitus}

A number of core treatment behaviors can be identified for those who are affected by the disease. Primarily, management should aim to improve healthy lifestyles, i.e. improving dietary patterns, to increase the level of physical activity, to maintain a healthy body weight, and should address smoking cessation (1). As insulin secretory capacities of the pancreas progressively decrease over time, or in the event of failing attempts to alter unfavorable lifestyles or to markedly reduce body weight, eventually many patients will require medical interventions. Oral hypoglycemic agents (OHAs) are generally considered primary medical interventions, with metformin being the most employed initial oral treatment globally and in the Netherlands $(1,13)$. Ultimately, patients may require a combination of oral medications, whether or not combined with insulin injections or other injectable drugs $(1,14,15)$. Most patients also need additional drugs to lower their blood pressure and cholesterol levels in order to reduce the elevated cardiovascular risk. The aim of the multifaceted treatment as described here is to achieve a favorable glucose metabolism, minimize CVD risk factors, improve the quality of life of those affected, and increase patients' life expectancy accordingly. The burden of this treatment can be quite high for an individual with T2DM but the management of diabetes can only be of optimal impact if patients adequately adhere to the agreed treatment behaviors.

\section{Adherence to treatment behaviors}

Patient adherence to core treatment behaviors poses a serious challenge. Despite the clinical benefits related to adherence to core treatment behaviors, i.e. achieving glycemic control, minimizing the risk of CVD, and living long and high-quality lives, patients' adherence is regularly suboptimal in practice (16). Treatment non-adherence not only affects the person with T2DM, but it is also associated with societal and political consequences, such as increased hospitalizations and healthcare expenditures $(7,17-22)$. The majority of patients does not consistently adhere to healthy lifestyle changes (23-25). Only a quarter to half of the adult population with T2DM achieves the recommended level of PA $(26,27)$. With regard to daily dietary recommendations, targets for protein, saturated fat, unsaturated fat, and fiber intake 
are achieved by less than $50 \%$ of the patients, respectively (26). A recent qualitative in-depth exploration of patients' eating pattern revealed that savory and sweet unhealthy snack intake emerged as major issue in their diet and accounted for a substantial amount of caloric intake. Health professionals also identified that unhealthy snack intake was highly prevalent in patients' diet (28). As a decreased caloric intake has been proposed as a means to improve the dietary pattern of patients with T2DM, targeting unhealthy snack intake could certainly contribute to this (1). Furthermore, across studies on adherence to oral hypoglycemic agents and insulin therapy, the adherence prevalence ranged from 39-93\%. Most of these reported a prevalence of adherence $<80 \%$, which is a commonly used threshold for unfavorable adherence levels $(29,30)$. Treatment non-adherence is generally not restricted to one treatment behavior, as less than $5 \%$ of the patients diagnosed with T2DM adheres to all treatment recommendations (i.e. smoking cessation, sufficient physical activity, sufficient fruit and vegetables consumption, moderate alcohol use, and maintaining a healthy Body Mass Index), and over $80 \%$ could make two or more behavior changes to improve treatment (25).

In sum, for most patients with T2DM it appears that non-adherence co-occurs across different treatment behaviors, contributing to poor glycemic control. Therefore, a multi-behavior approach covering healthy lifestyle and medical behaviors in targeting treatment nonadherence is relevant. In order to understand adherence behavior and to identify targets for intervention, it is of primary importance to identify determinants of those behaviors, as described below.

\section{Understanding adherence to healthy lifestyle and medical behaviors}

In order to develop new and effective strategies that help to improve adherence to healthy lifestyle and medical behaviors, it is essential to explore and understand what determines patient adherence to these behaviors. Once knowledge about relevant determinants is established, programs can be developed accordingly by addressing these factors, and thereby increasing the likelihood that intended improvements in adherence concerning healthy lifestyle and medical behaviors are achieved (31). A multitude of factors interact simultaneously in the process of (non)-adherence including social and economic factors, the healthcare system, characteristics of the disease and therapy, and patient-related factors $(32,33)$. Although all these factors provide entries for targeting treatment non-adherence, most are difficult to change, and while if changed, they may only be of indirect impact on adherence through perceptions of the patient (33). As patients are considered largely selfresponsible for their level of adherence, and individual factors account for the large majority of the variance observed in glycemic control, patient-related factors seem to be the most viable and changeable intervention domain $(33,34)$. 
Health behavior theories and models can guide the process of exploring and understanding individuals' engagement in health behaviors. Various theories and models are available, such as the Social Cognitive Theory (35), the Trans Theoretical Model (36), the Health Belief Model (37), and the successive Theories of Reasoned Action and Planned Behavior (38, 39). Most of those described behavior change as a deliberate or rational process and mainly incorporated personal-related beliefs. Existing models and theories have been able to explain variance in diabetes-related health behaviors to some extent $(40,41)$, but have also been subject to substantial criticism (42). The criticism largely focusses amongst others on the predictive validity of the models, its reductionism, and the importance of addressing different phases of behavior change (43). It is acknowledged that the vast majority of explained variance is not accounted for by constructs included in such theories, that the quantity of constructs may be insufficiently elaborated to explain behavior that is assumed to be volitional, and that various phases of behavior change can be distinguished $(42,44)$. For example, the Theory of Planned Behavior assumes that that motivational factors, i.e. attitudes, subjective norms and perceived behavioral control, are predictive for someone's intention to perform a health behavior, and that the intention is predictive for actual behavior (39). However, the theory less explicitly dedicates attention to the determinants preceding the formation of motivation. Research has, for instance, shown that factors such as knowledge and risk perceptions may play an important role as factors determining motivational factors such as attitudes and self-efficacy (45). Additionally, only a limited amount of the variance of behavior is explained by intention, and therefore the role of developing and enacting action plans should be addressed in order to understand and change behavior (46-50).

A way to proceed may be to integrate available theories and models, e.g. by maintaining their strengths and eliminating or refining their pitfalls. An example of such an integrative model is the integrated model for explaining motivational and behavioral change, shortly called the I-Change Model (43). The I-Change Model (figure 1) integrates various well-known sociocognitive theories, e.g. the Social Cognitive Theory (35), the Trans Theoretical Model (36), the Health Belief Model (37), and the Theories of Reasoned Action and Planned Behavior (38, 39). The I-Change Model has been successfully applied to map salient beliefs of health behavior (change) and to develop change programs on a wide range of health-related behaviors (51, 52). The I-Change Model is applied in this dissertation as theoretical framework to explore and understand (non)-adherence and to guide the process of improving non-adherence to core T2DM treatment behaviors. 
Figure 1 The I-Change Model as applied in this dissertation, adapted from (de Vries, 2017)

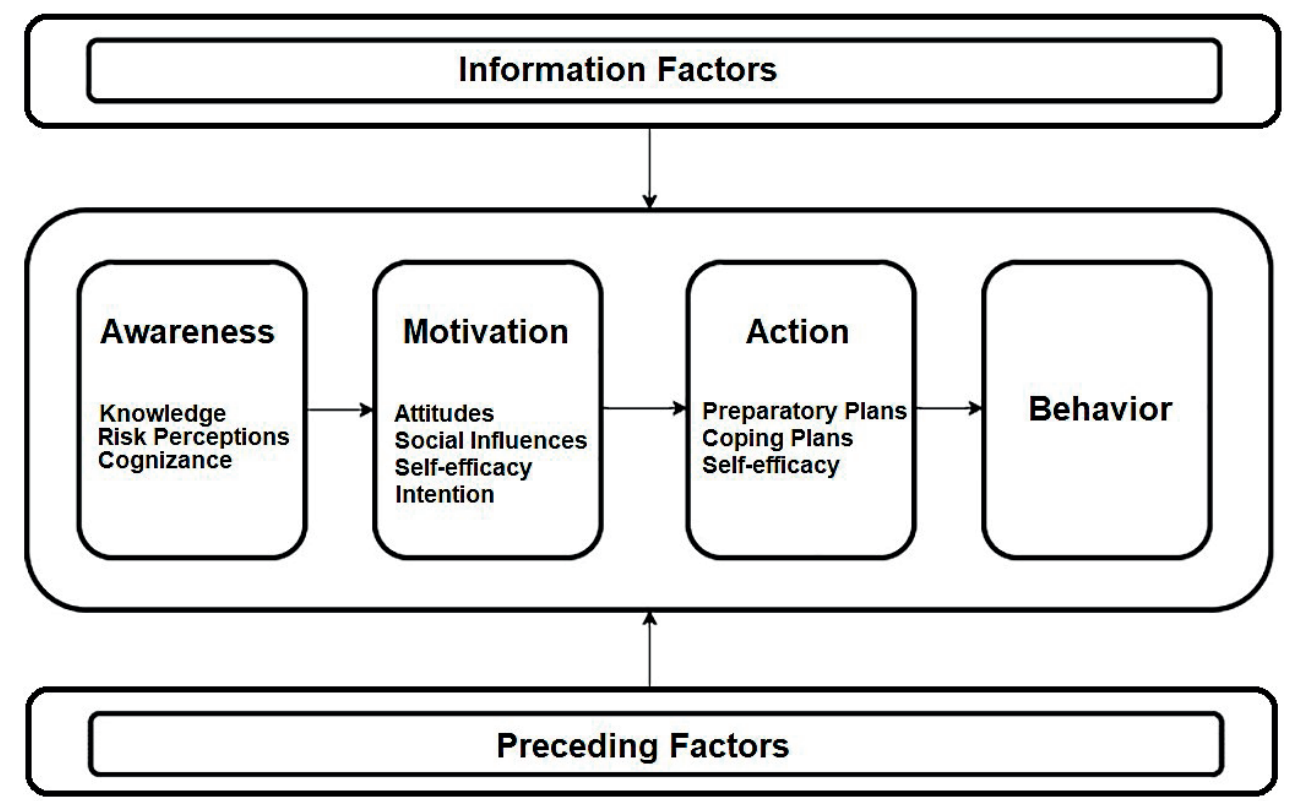

\section{Theoretical Framework: The I-Change Model}

The core of the I-Change Model distinguishes between a pre-motivational, motivational, and post-motivational phase, which are moderated by information factors, i.e. personal, message, channel and source factors, and preceding factors, i.e. biological, psychological, behavioral, and environmental factors. The model posits that an individual first needs to be aware of the necessity of a change in behavior in order to create a sufficient level of motivation to cognitively process new information regarding the pursued behavior change. Awareness can be obtained by acquiring sufficient knowledge of the topic (e.g. what defines treatment adherence), by recognizing the possibility and severity of consequences due to treatment non-adherence (e.g. feelings of susceptibility and severity of the negative consequences of hyperglycemia due to medication non-adherence), and cognizance (e.g. whether the patient can adequately estimate if they are currently adherent or not). The model postulates that such pre-motivational factors play a more distal role, i.e. an indirect relation to behavior is assumed through factors in the motivational phase. This means that patients first need to become aware of the necessity of behavior change, in order to form motivation (45). Motivational factors in the model include factors also acknowledged by other social cognitive theories such as attitudes, social influences and self-efficacy. Attitudes entail a weighing of positive and negative rational and emotional beliefs towards the pursued behavior (e.g. pros and cons of engaging in physical activity). Social influences include perceptions of social support, norms and modeling behavior of relevant others (53), and self-efficacy entails one's perceived 
ability to successfully enact behavior under tempting circumstances (e.g. being physically active when it rains) $(54,55)$. Together, these motivational factors determine the intention a person has to change a certain behavior.

Once a high intention to change behavior is present, individuals can facilitate behavior change by making preparatory and coping plans and applying these plans in practice, as research shows that a high intention does not automatically warrants change $(56,57)$. For instance, an individual could prepare engaging in physical activity by planning where, when, and how they will be active. By anticipating challenging situations where the intended behavior change is likely to be difficult to adhere to (e.g. when having a busy day or when being ill) and making a plan to deal with the situation (e.g. instead of eating in the cafeteria, the patient intends to eat while having a walk instead), individuals can increase the likelihood of successfully carrying out the intended behavior, despite these personal challenges (47).

\section{Improving patient treatment adherence}

Once it is defined which treatment behaviors of a patient could be improved and relevant information on the determinants of those behaviors is obtained, programs can be initiated accordingly $(58,59)$. In the planning phase of the design of a program that aims to improve patient adherence, it is of importance to explore the various avenues how such a program could be delivered. For instance, it is not only relevant to show effectiveness, but also that as many people of the target population as possible can be reached in order to maximize program impact (60).

Consultations between health professionals and patients seem to provide a viable platform to address the issue of non-adherence and to discuss how to improve adherence. In the Netherlands, healthcare reaches most patients affected by T2DM as they generally visit their health professional every three to six months for a consultation of approximately 20-30 minutes. During these sessions, many standard topics should ideally be discussed, according to established protocols, such as the patient's well-being, glycemic control, complaints and complications, as well as therapy adherence (13).

Practice nurses and diabetes nurses, who are the primary contact point of care of T2DM patients in the Netherlands, seem to be in an ideal position to address the issue of treatment non-adherence and to align strategies on how to improve adherence $(14,61)$. Despite acknowledging the importance of treatment adherence, scarce and busy consultations often impede health professionals from discussing this complex topic $(14,62)$. Moreover, research suggests that health professionals lack knowledge, motivation, and confidence to address (non)-adherence and to promote healthy lifestyles during these consultations $(63,64)$. As 
patients still require support to self-manage their illness and related treatment behaviors, alternative program delivery strategies need to be explored.

Multiple patient-focused programs are available that aim to tackle the issue of treatment non-adherence in patients with T2DM. These programs are heterogeneous regarding their intervention strategy, design, setting, population, and outcomes. The vast majority of these programs targets solely improvements in adherence to medical behaviors, while it is wellknown that in order to achieve glycemic control, also adherence to healthy lifestyle behaviors is needed $(33,65-67)$. As a consequence, most programs either show small or no effects (33, 68). Moreover, as non-adherence frequently co-occurs across distinct core T2DM treatment behaviors, a program that targets a combination of both healthy lifestyle and medical behaviors through a multi-behavior approach, might therefore be more likely to be effective (25). Next to such a multi-behavior approach, other factors may enhance the success of programs that target improvements in adherence, such as the delivery of the program through the Internet.

\section{Delivery of behavior change interventions through the Internet}

The Internet has widely and successfully been applied for health services and health promotion activities such as the management of T2DM; such activities delivered through the Internet are often referred to as eHealth $(65,69-72)$. eHealth applications have shown to be (cost)-effective, easy in use, and can temper pressure on the healthcare system, for instance by outsourcing activities or tasks of health professionals to the Internet (73-78). In addition, unlike face-to-face interventions such as regular consultations with a healthcare professional, advantages of eHealth programs include their broad reach, i.e. their potential to reach large audiences of people, the ongoing support of the self-management process outside the clinical setting through its constant availability, and they may free health professionals to shift their focus to other priorities $(71,79)$.

The success of eHealth programs, also in line with factors determining the effectiveness of programs in general, depends on the application of a solid theoretical framework, the possibility to identify behaviors at risk, the application of goal-setting and possibilities for self-monitoring, the incorporation of interactive, tailored, and visual supported content, and focusing and aligning on distinct behavior change phases, i.e. awareness, motivation, and self-regulation $(65,70,71,79,80)$. Despite acknowledging a wide variety of success factors for eHealth programs, a review in 2014 on eHealth programs supporting T2DM management (71) concluded that only one of the nine included studies reported significant improvements in dietary behavior and PA; no effects were observed for medical behavior adherence (79). Generally, existing eHealth programs related to the management of T2DM often include little interactive content and tailored strategies, are mainly text-based, do not employ a theoretical foundation and technology, and focus on separate behaviors that play a role in the management of T2DM instead of combining behaviors $(65,70,71,80)$. In sum, eHealth does provide a viable option to improve treatment behavior adherence in patients with T2DM. So 
far, however, success factors have not been adequately applied which may explain the poor results of existing programs. Hence, eHealth initiatives targeted at T2DM treatment adherence can be significantly improved by building on a theoretical framework, including interactive and visual supported content, applying a multi-behavior approach, and incorporating risk identification, goal-setting, and adherence monitoring facilities. Moreover, programs could be improved by the application of computer-tailoring technology; a strategy that can yield highly personalized advice to a patient and relevant information aligned to the person's behavior change phase (81).

\section{Computer-tailoring technology}

Patients with T2DM generally show non-adherence in multiple behaviors, but the specific behaviors most suitable for improvement may differ among individuals (25). For instance, an individual may be more willing or confident to improve a particular behavior, compared to another. This emphasizes the need for programs that are aligned to the particular profile of an individual and a generic approach will usually not fit all when multiple behaviors are targeted (82). The strategy of adapting a program to the characteristics and needs of the user by asking questions and using the responses to generate tailored computer driven advices is referred to as computer-tailoring $(81,82)$. Compared to generic programs, computer-tailored programs are more likely to be perceived as personally relevant and to stimulate greater cognitive activity in users (83). Computer-tailored content is based on unique answers given on a webbased assessment. Such an assessment consists for example of questions on adherence levels of the person regarding behavior (e.g. levels of adherence to mediation or physical activity guideline targets), demographic characteristics (e.g. gender, age, education level, and comorbidity), and socio-cognitive variables (e.g. knowledge, attitudes, and intention towards behavior change). The answers to such questions are processed in a computer system that links individual or combined answers to pre-formulated health messages. Tailoring algorithms link the individual's responses provided to a questionnaire to appropriate advice messages and thus result in the delivery of highly tailored and relevant web-based advice. Consequently, individualized content is presented which in a way mimics the counseling process of healthcare professionals counselling their patients (81).

\section{The development and evaluation of 'My Diabetes Profile'}

The aim of the project described in this dissertation was to develop and evaluate a webbased computer-tailored program for patients with T2DM in order to improve adherence to healthy lifestyle and medical behaviors, i.e. improving PA levels, decreasing caloric intake from unhealthy snacks, and increasing adherence to OHAs and insulin therapy $(14,15)$. This project consisted of several phases. 
First, explorative studies were conducted in order to identify which treatment behaviors were subject to improvement in patients with T2DM and which specific determinants within those treatment behaviors were associated with (non)-adherence. Exploring salient beliefs, which are the cognitive representation of determinants, is essential to understand what determines those behaviors. Moreover, this information is important to know which specific feedback messages were needed for this population and in order to construct programs that are likely to be successful in behavior change $(31,38,84)$. In fact, at the level of beliefs, salient information should be obtained about the considerations that lead people to engage or not engage in a given health behavior $(84,85)$. Besides the conduct of explorative studies, relevant information was gathered through studying recent Internet programs that examined the effect of computer-tailoring technology on improving adherence to multiple behaviors. The outcome of the explorative studies, which consisted of both qualitative and quantitative research, is presented in chapters 2 and 3.

Second, an advisory committee was formed prior to the development of the program, in order to discuss its content, tailoring strategy, and to align the program with the needs and wishes of the target group. The committee included both delegates from the target population, i.e. patients with T2DM, as well as local experts in the field of healthcare, computer-tailoring technology and health behavior change.

Third, we developed 'My Diabetes Profile', a program that integrates the factors which were identified to determine success of eHealth programs aimed at improving treatment adherence and which was theoretically grounded in the I-Change Model (43). The program consists of several subsequent sessions, i.e. a health risk appraisal session, an awareness and motivation session, and a goal-setting, monitoring and action planning session. Throughout these sessions, individuals receive personally relevant information on risk behaviors and relevant content tailored to various phases of behavior change which are addressed in the I-Change Model. In addition, the tailored content is offered through video-, visual- and brief text-tailored advice. Furthermore, the program focuses on multiple behaviors involved in the management of T2DM in a sequential matter. Specific elements of the program, for example the videos, navigation tools, and its usability were pragmatically tested in our target group. Conducting such usability evaluations are a pivotal step in the development of programs in order to make pre-trial improvements, and to assess patients' satisfaction with the program (86). Improvements were made based upon these usability evaluations, that is, instructions were included on how to answer certain questions, important text words and fragments were highlighted to raise awareness and cognitive processing, and clear directions were included on how to navigate in the program.

Fourth, we developed a protocol for a randomized controlled clinical trial (RCT) to determine the effectiveness of the My Diabetes Profile in patients with T2DM. In an RCT, which is generally considered the golden standard in conducting effectiveness research (87), participants are 
randomly allocated to an intervention or control arm and compared in terms of a selected outcome measure, e.g. adherence to treatment behavior (88). Assessments were conducted at baseline, i.e. just before the six-month program access period, and directly after the access period. Outcomes compared between both trial arms comprised of overall adherence behavior change, i.e. a composite score of changes in adherence to separate core treatment behaviors, and - as a secondary outcome - a score for the change in each separate adherence behavior. An extensive description of the program, including its development and content, as well as a protocol for conducting the effectiveness trial is described in chapter 4 of this dissertation.

Fifth, a multi-center RCT was performed, in both general practice and outpatient clinics. For a period of six months, patients with T2DM were recruited by their practice nurse or diabetes nurse, and randomly allocated to either the intervention arm (receiving program access) or the control arm (receiving no program access, waiting list) for six months. When conducting an RCT in order to examine the effectiveness of a program, large sample sizes are often required to warrant sufficient study power. In our trial, as well as in many other trials, patient recruitment was based on the efforts of health professionals, given their direct contact and trustful relationship with their patients $(89,90)$. Recruiting the required sample size for randomized controlled trails can be a challenge, but is essential to successful conduct of clinical trials (91). Many trials do not reach their required number of patients, which can jeopardize generalizability of observed results, reduce study efficiency due to extended recruitment periods, and lead to premature discontinuation of trials (90, 92-94). Moreover, the dissemination of possible effective treatments to a broad population may be delayed (95). Researchers have been called to thoroughly evaluate their recruitment procedure applied in trials, as current research highlights the paucity of conclusive evidence regarding factors influencing recruitment success $(91,96)$. In our trial, nurses across the Netherlands were approached in order to recruit at least eight patients with T2DM to our trial. Once all patients were included in the trial, this recruitment process was evaluated by exploring factors influencing nurses' recruitment and the results of this evaluation are presented in chapter 5 . The results of the RCT are described and discussed in chapter 6 of this dissertation.

\section{Aim and outline of dissertation}

The main aim of this dissertation was to develop and subsequently provide insight in the effectiveness of the eHealth program, My Diabetes Profile, which aimed to improve adherence of patients with T2DM to core treatment behaviors, i.e. improving physical activity levels, oral hypoglycemic agent adherence, insulin therapy adherence, and decreasing caloric intake from unhealthy snacks. This dissertation is divided into two parts which subsequently contribute to its overall aim, and is concluded with a general discussion (Chapter 7). 


\section{Part I: Formative research, program development and trial protocol}

Chapter 2: Exploring beliefs on diabetes treatment adherence among Dutch type 2 diabetes patients and healthcare providers.

Chapter 3: Psychological predictors of adherence to oral hypoglycemic agents: An application of the ProMAS questionnaire.

Chapter 4: A web-based program to improve treatment adherence in patients with type 2 diabetes: Development and study protocol.

\section{Part II: Recruitment and effectiveness evaluation}

Chapter 5: Exploring factors influencing recruitment success of nurses recruiting diabetes patients for a randomized controlled trial.

Chapter 6: The effectiveness of a web-based computer-tailored program to improve treatment adherence in patients with type 2 diabetes: A randomized controlled trial. 


$$
1
$$




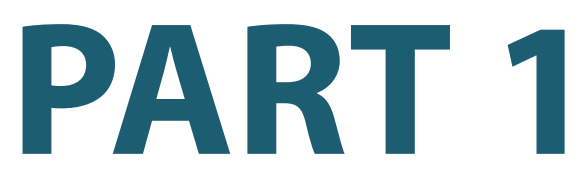

Formative research, program development and trial protocol 


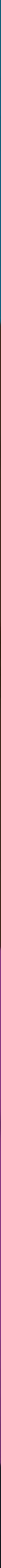




\section{Chapter 2}

\section{Exploring beliefs on diabetes treatment adherence among Dutch type 2 diabetes patients and healthcare providers}

\section{This chapter has been published as:}

Vluggen S, Hoving C, Schaper NC, de Vries H. Exploring beliefs on diabetes treatment adherence among Dutch type 2 diabetes patients and healthcare providers. 


\section{Abstract}

Objective: Despite well-known beneficial effects, adherence to core elements of diabetes treatment is suboptimal. This study, conducted in the Netherlands, aimed to explore if and how treatment adherence success factors are applied in diabetes consultations, and to explore salient personal beliefs about type 2 diabetes treatment including both healthy lifestyle adaptations and pharmacotherapy.

Methods: A qualitative study using semi-structured interviews among nine Dutch healthcare providers predominantly involved in diabetes management and 19 Dutch type 2 diabetes patients. Data was systematically analyzed through deductive coding analysis using Nvivo.

Results: Most patients visited their consultations unprepared. Patients did not or vaguely experience goal-setting in consultations, whereas healthcare providers indicated to set treatment goals. Shared-decision making was applied, however patients were rather passive collaborators as mostly healthcare providers were in charge of making treatment decisions. Despite suboptimal treatment adherence, many advantages and few disadvantages of treatment strategies were reported. Adherence self-efficacy was lower in situations outside daily routine.

Conclusion: Treatment adherence success factors are not optimally applied, and in particular treatment adherence self-efficacy could be improved.

Practice implications: The application of treatment adherence success factors in consultations could be improved, and personal beliefs should be addressed to improve treatment adherence and optimize counselling. 


\section{Introduction}

Type 2 diabetes mellitus (T2DM) is a chronic progressive disease characterized by hyperglycaemia and the body's inability to retain an optimal glucose metabolism $(97,98)$. Worldwide nearly 400 million people live with T2DM, with expectations of almost 600 million people being affected by 2035 (98). This rise is largely attributable to unhealthy energy balance-related behaviours such as physical inactivity and unhealthy dietary patterns (9799). Core T2DM treatment elements, which target an optimal glucose metabolism, consist of adaptations towards healthy lifestyles, i.e. increasing physical activity (PA) and improving dietary patterns, and pharmacotherapy, i.e. taking glucose lowering pills and/or injecting insulin (100). Despite the well-known long-term benefits of adequate glycaemic control on reducing microvascular complications and death from any cause (16), patients' treatment adherence is suboptimal (101-103). Several studies show that the majority of T2DM patients does not meet recommended levels of PA and does not adhere to dietary guidelines (23, 24). Moreover, King et al. (25) showed that over $95 \%$ of diabetes patients failed to adhere to all five healthy lifestyle recommendations studied. Adherence prevalences on diabetes pharmacotherapy vary across studies from 38.5 to $93.1 \%$, far most of them reporting prevalences $<80 \%$ (30). Poor adherence not only impedes beneficial treatment effects, but is also associated with disease worsening, cardiovascular risks, a reduced quality of life, more hospitalizations, increased healthcare costs, and premature mortality $(7,18-22,104)$.

Treatment adherence implies collaboration between a healthcare provider (HCP) and a patient in the formulation and agreement of treatment strategies. This collaborative care strategy can be applied in consultations, which should serve as a setting where patients are informed, educated, facilitated, motivated, and taught skills to optimally self-manage T2DM in daily life (105). Determinants of patient treatment adherence have been identified at both HCP and patient level. At the HCP level, knowledge insufficiencies, lack of time and motivation, and difficulties in involving patients in decision-making have been identified as adherence barriers (63). Patients on the other hand often lack knowledge and motivation to change lifestyles, and discipline to persist adapted lifestyles $(14,63,64)$. Moreover, French et al. (84) demonstrate that personal beliefs about treatment play an important role in treatment adherence, especially regarding the core elements of T2DM treatment. To ensure treatment success and adequate glycaemic control, active patient engagement, applying goal setting principles, tailoring treatment strategies to patients' needs, beliefs and abilities, involving patients in decision-making, and effective ongoing self-management beyond consultations is required $(15,64,104,106)$. Because the basis for treatment strategies originates from consultations between HCPs and patients, and as still many patients suboptimally adhere to their treatment, it is evident to profoundly explore if and how treatment adherence success factors are applied in consultations. Moreover, it is important to explore salient personal beliefs about core treatment elements as they play an important role in treatment adherence. 
A better understanding of these factors can offer handles to optimize treatment adherence and improve T2DM counselling (84).

The aim of this study was to: (1) explore if and how treatment adherence success factors are applied in consultations between HCPs and T2DM patients, and (2) explore salient personal beliefs about T2DM treatment, including both healthy lifestyle adaptations and pharmacotherapy among HCPs and T2DM patients.

\section{Methods}

\section{Procedure}

We conducted a qualitative study using semi-structured individual interviews among HCPs predominantly involved in T2DM management and T2DM patients. The study was approved by the ethics committee of the MUMC+ (15-4-094).

HCPs and patients were recruited from July up to November 2015 in the southern region of the Netherlands. Purposefully efforts were made to recruit a heterogeneous sample in terms of age, gender, education level, and healthcare setting. A priori, we aimed to conduct 25 interviews among fifteen T2DM patients and ten HCPs: our initial analysis sample (107). This number was chosen because both perspectives needed to yield sufficient representative results. We aimed to include more patients, as the absolute number of patients is much larger than the actual number of HCPs. Moreover, we thought that HCPs would carry many experiences of consultations with patients, whereas patients would only have their own experience. After these 25 initial interviews, two additional patient interviews and one additional professional interview were conducted to confirm saturation and content validity for both interviewed groups (107). No new themes emerged from these additional interviews, hence data saturation was reached. The inclusion criterion for HCPs was: having treated T2DM patients in the Netherlands at least once in the last three months. Further we included general practitioners, practice nurses, internists, diabetes nurses, and dieticians. In the Netherlands, these professionals are predominantly involved in T2DM management and in shaping treatment strategies, hence these HCPs were included (14). HCPs were recruited through e-mail, including a non-committal study description and a participation request. In case of participation agreement, an interview date was picked, and HCPs provided written informed consent. HCPs were interviewed by the first author at the HCPs' workplace. Afterwards, they received a symbolic diabetes-related reward. Five general practices and one hospital were approached to recruit patients. Eligible patients were identified by the HCP and inclusion criteria were: T2DM diagnosis for at least one year, 40-70 years old, receiving treatment in the Netherlands, and using at least one form of glucose lowering medication. Patients not able to speak and understand the Dutch language and patients unable to use a computer were excluded. Eligible patients were approached by their HCP to explain study details, including a 
non-committal information letter with a study description, and a participation request. In case of willingness to participate, s/he could contact the researcher, an interview date was picked, and patients provided written informed consent. Patients were interviewed at the research institute by the first author and rewarded with a $€ 25$,- gift card.

\section{Measurement}

All interviews were structured using an interview route. Various experts involved in T2DM management and T2DM patients provided feedback on the interview route and adaptations were made resulting in a final version consisting of four topics: consultations between HCPs and patients, salient personal beliefs about T2DM treatment, diabetes literacy, and eHealth. Diabetes literacy and eHealth are not further elaborated in this manuscript. Interviews started with identifying demographic characteristics. The I-Change Model (figure 1) (108) was used to theoretically inform this study. The model distinguishes an awareness, motivation, and action phase in explaining and changing behaviour, which are influenced by preceding and information factors. It incorporates various socio-cognitive theories $(36,37,109)$ and has been used frequently to identify beliefs involved in health-related behaviour (110). Consultations between HCPs and patients focussed on treatment adherence success factors such as goalsetting, shared-decision making (SDM), and self-management (education). These factors fall under 'information factors' in the I-Change Model (108). Moreover, the I-Change Model incorporates several determinants of treatment adherence which have been identified earlier like knowledge, attitudes, self-efficacy, and action planning $(14,63,64)$. These determinants were operationalized (table 1) to identify underlying salient personal beliefs of core treatment elements. The identification of these beliefs focussed mainly on determinants in the awareness and motivation phase. 
Figure 1 The I-Change Model

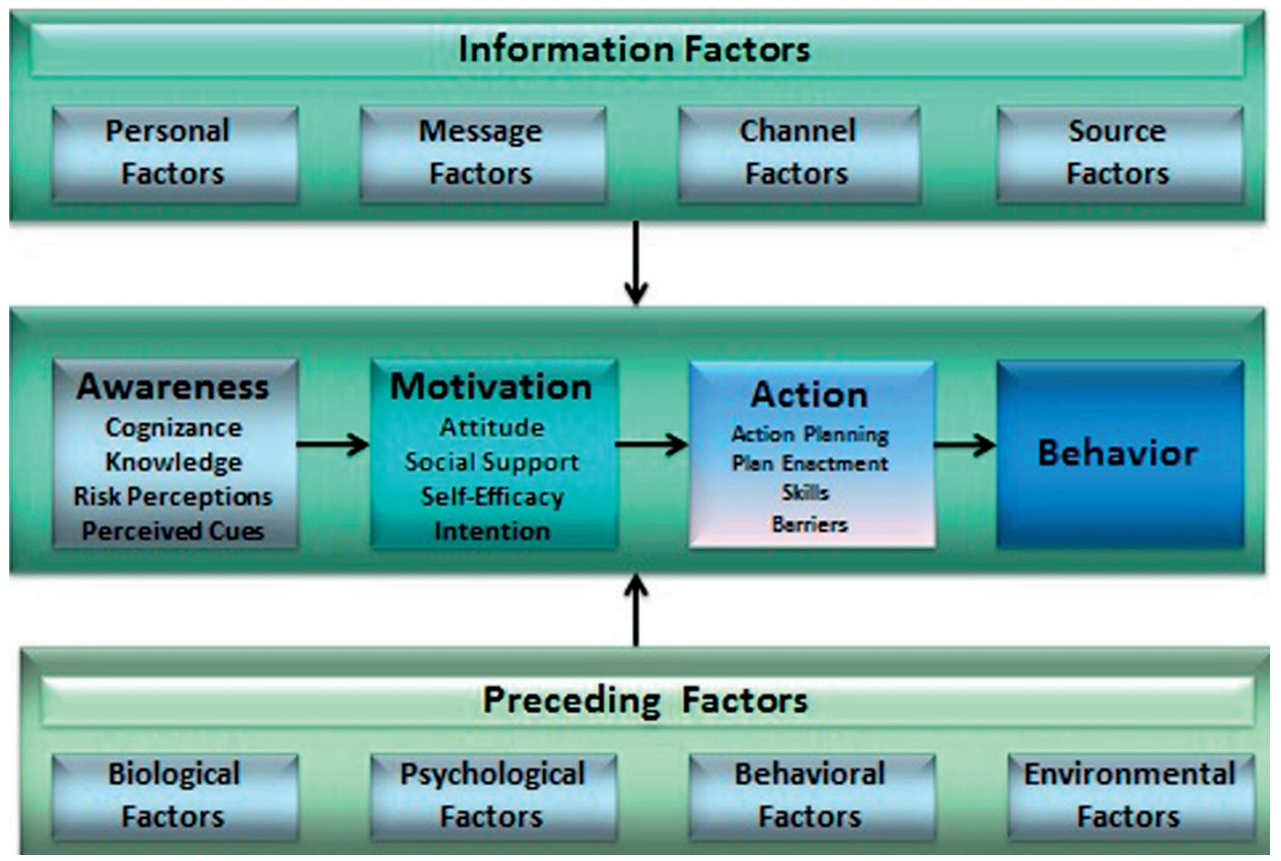

Table 1 Interview questions: operationalization I-Change Model concepts

\begin{tabular}{ll}
\hline I-Change Model Concept & Operationalization \\
\hline Information Factors & What is the course and content of a consultation meeting? \\
& Do patients prepare their consultation, and how? \\
& Are treatment strategies and goals formulated, and how? \\
& What is the role of the patient and HCP in a consultation, and (how) is \\
& shared-decision making applied? \\
& What is a direct trigger for you to be physically active? \\
Perceived Cues & Do you (think) you adhere to your medication regimen? \\
Cognizance & What is the effect/mode of action of your medication? \\
Knowledge & Which are (un)healthy foods? \\
& What could happen if you don't use your medication, and how severe \\
Risk Perceptions & would you find this? \\
& What could be consequences of being physically inactive, and how \\
& severe would you find this? \\
Attitudes & What are advantages for you of a healthy diet? \\
Social Influences & What are disadvantages for you of being physically active? \\
& Who supports you to use your medication? \\
Self-efficacy & Are there people who think you should eat healthy? \\
Intention & In which situations is it more difficult for you to be physically active? \\
Action Planning & Are you planning on improving your medication adherence? \\
Coping Planning & Which plans do you have to increase your physical activity? \\
& Which coping plans do you have to eat healthier when facing a \\
& difficult situation?
\end{tabular}




\section{Analysis}

Interviews were audio recorded, transcribed verbatim, anonymized, and analysed using Nvivo 10 (111). Deductive coding analysis (112) was performed by two researchers; the first author and a research assistant. For HCPs and patients separately, a pre-defined coding tree was independently applied to a single random transcript by both researchers. Interview passages were assigned codes, and the creation of additional codes and inconsistencies were discussed and agreed upon. The same process was then repeated on three other random transcripts. This resulted in a final coding tree which was then applied to analyse four random HCPs transcripts and four patients' transcripts by both researchers (112). Subsequently, the intercoder reliability and Cohen's Kappa were assessed using the coding comparison query. For both HCPs and patients, this resulted in a percent agreement of 0.99 , reflecting a high degree to which interview passages were assigned to the same codes by both researchers. The Cohen's Kappa showed to be 0.67 for the HCPs group and 0.79 for the patients group, reflecting a good strength of intercoder agreement $(112,113)$.

\section{Results}

\section{Sample characteristics}

Thirty-one participants agreed on study participation: nine HCPs and 22 patients. Three patients were excluded because they did not meet the inclusion criteria. Five interviews were conducted in two parts because of time constraints of participants. HCP interviews were conducted with general practitioners ( $G P, N=2)$, practice nurses ( $P N, N=2)$, internists (INT, $\mathrm{N}=1$ ), diabetes nurses ( $\mathrm{DN}, \mathrm{N}=2$ ), and dieticians ( $\mathrm{DIET}, \mathrm{N}=2$ ). Interviews with $\mathrm{HCPs}$ lasted on average 61 minutes, ranging from 50-75 minutes. Interviews with patients lasted on average 82 minutes, ranging from 59-107 minutes. Patients had on average a suboptimal HbA1c-level of $63 \mathrm{mmol} / \mathrm{mol}$, and were on average obese $\left(B M l=35.4 \mathrm{~kg} / \mathrm{m}^{2}\right)$. Sample characteristics are shown in table 2.

Table 2 Sample characteristics interviewees

\begin{tabular}{|c|c|c|}
\hline & $\mathbf{N}(\%)$ & Mean (SD) \\
\hline \multicolumn{3}{|c|}{ T2DM Patient Characteristics } \\
\hline \multicolumn{3}{|l|}{ Gender } \\
\hline Male & $10(52.6)$ & \\
\hline Female & $9(47.4)$ & \\
\hline \multicolumn{3}{|c|}{ Education level } \\
\hline High & $6(31.6)$ & \\
\hline Low & $13(68.4)$ & \\
\hline
\end{tabular}


Table 2 Sample characteristics interviewees

\begin{tabular}{|c|c|c|}
\hline & N (\%) & Mean (SD) \\
\hline \multicolumn{3}{|l|}{ Marital Status } \\
\hline Single & $4(21.1)$ & \\
\hline In a relationship & $15(78.9)$ & \\
\hline \multicolumn{3}{|l|}{ Pharmacotherapy } \\
\hline Glucose lowering pills & $6(31.6)$ & \\
\hline Insulin & $3(15.8)$ & \\
\hline Combined & $10(52.6)$ & \\
\hline Age & & $57.3(7.1)$ \\
\hline $\mathrm{HbA}_{1 \mathrm{c}}(\mathrm{mmol} / \mathrm{mol})^{\mathrm{a} / \mathrm{b}}$ & & $63^{\mathrm{a}}(18.1)$ \\
\hline BMI $\left(\mathrm{kg} / \mathrm{m}^{2}\right)^{\mathrm{b}}$ & & $35.4(6.7)$ \\
\hline T2DM Duration (years) & & $12.7(8.6)$ \\
\hline Interview Duration (min.) & & $82(17.3)$ \\
\hline \multicolumn{3}{|l|}{ HCPs' Characteristics } \\
\hline \multicolumn{3}{|l|}{ Gender } \\
\hline Male & $3(33.3)$ & \\
\hline Female & $6(66.7)$ & \\
\hline \multicolumn{3}{|l|}{ Work Setting } \\
\hline Primary Healthcare & $5(55.6)$ & \\
\hline Secondary Healthcare & $4(44.4)$ & \\
\hline Age & & $47.3(11.4)$ \\
\hline Interview Duration (min.) & & $60.6(7.8)$ \\
\hline
\end{tabular}

BMl body mass index, $\mathrm{HbA}_{1 c}$ glycosylated haemoglobin, SD standard deviation,

a This equals an $\mathrm{HbA}_{1 c}$ of $7.9 \%$

${ }^{b}$ Target values for $\mathrm{HbA}_{1 \mathrm{c}}$ and $\mathrm{BMI}$ are $<53 \mathrm{mmol} / \mathrm{mol}$ and $<25 \mathrm{~kg} / \mathrm{m}^{2}$ respectively.

\section{Diabetes consultations}

\section{Course and content}

Most patients indicated to visit their practice nurse or diabetes nurse quarterly, their general practitioner or internist once a year, and a dietician if appropriate. All HCPs reported that the course and content of those consultations was highly protocolled, meaning that several topics like discussing blood tests results, lifestyle and medication (adherence), and complication management would recur every consultation. Moreover, if applicable, time was devoted to issues raised by patients like questions and personal demands of care. Little time was spent on self-management education [Quote \#1]. Most HCPs and patients reported that patients did not prepare their consultations. Patients who did so, reported activities such as recording blood glucose levels and writing down questions. HCPs stated that they would welcome a 
more active participation and disease engagement of their patients, as many were passively oriented. Few HCPs actually tried to stimulate their patients to actively participate [Quote \#2].

\section{Goal Setting}

Most patients indicated that no goals were set regarding treatment strategies. Patients reported that if goals were set, they were often vague and perceived as advice rather than goals [Quote \#3]. In contrast to patients' perceptions, most HCPs reported that they often formulated goals during consultations, especially concerning lifestyle changes, improving glycemic control and treatment adherence, and stabilizing disease worsening. Most HCPs indicated to tailor goals to patients' needs and abilities by proposing small steps and realistic goals [Quote \#4]. Some HCPs reported that treatment goals were often not reached due to a lack of motivation in patients. According to some HCPs, patients sometimes aimed for higher, unachievable goals [Quote \#5].

\section{Shared-Decision Making}

A small majority of HCPs and patients indicated that the course and content of consultations, as well as the formulation of treatment strategies and goals, were determined by the HCP and patient in conjunction. This manifested itself either in patients who passively agreed on subjects and treatment strategies proposed by their HCP, justified by 'the HCP knows best', or through a few patients who came up with treatment ideas themselves. HCPs perceived that older patients were more dependent on the HCP's approach and younger patients were seen as more active collaborators through bringing up treatment ideas themselves and searching information on the Internet [Quotes \#6 and \#7]. Many HCPs indicated that treatment adherence was the patient' own responsibility. Contrary to the overall passive engagement in the formulation of treatment strategies, all patients felt they were responsible for treatment adherence.

\section{Adherence to treatment elements}

Many patients stated to be physically active and only few stated to be fully inactive. Often mentioned activities which contributed to PA were: walking (the dog/upstairs), cycling, shopping, household activities, and visiting a physiotherapist or gym. However, according to almost all HCPs, adherence to PA guidelines was poor [Quote \#8]. According to most HCPs, adherence to healthy diets was poor. Issues identified focused on irregularity of meals, portion size and snacking in between and/or after regular meals. In general, patients indicated to maintain a healthy diet. However, when asking for detailed diet description, emphatically intense savory and sugary snacking behavior in between or after regular meals could be identified in most patients [Quotes \#9 and \#10]. Near all HCPs reported that adherence to pharmacotherapy in patients was suboptimal. Both intentional (purposely skipping or altering regimes) and unintentional (forgetting) non-adherence underlie this statement. Half of the patients indicated to always adhere to their diabetes pharmacotherapy. Regarding adherence to glucose lowering pills and insulin, similar types of non-adherence emerged like forgetting, 
skipping, stopping, stacking medication, adapting dosages, and using medication at varying times [Quotes \#11 and \#12].

\section{Awareness beliefs}

HCPs believed that patients' knowledge levels regarding health benefits of PA were sufficient. Almost all patients believed that regular PA should be part of a healthy lifestyle. When discussing risks of PA non-adherence, patients stated risk of weight gain, loss of fitness, becoming less mobile, getting hyperglycemia, and developing cardiovascular diseases [Quote \#13]. Regarding a healthy diet, HCPs indicated that patients were aware of the fact that they should eat less and healthier, but that they were less aware of the products which did and did not contain carbohydrates. Furthermore, HCPs encountered patients who believed that treatment involved a no-sugar diet. In contrast to HCPs perceptions, most patients stated to be aware of what carbohydrates are, and which products contained them. Patients believed that an unhealthy diet could result in hyperglycemia, cardiovascular diseases, weight gain and an increase of pharmacotherapy. Most HCPs stated that the majority of patients had insufficient knowledge regarding their pharmacotherapy. This manifested itself in not knowing which pills were glucose lowering pills, or being unfamiliar with their mode of action. HCPs indicated to make patients aware of the susceptibility of getting complications as a result of pharmacotherapy non-adherence [Quote \#14]. Most patients could not or only vaguely explain the mode of action of their medication. Almost all patients stated that nonadherence would result in hyperglycemia, and almost half of them associated non-adherence with long-term consequences like myocardial infarction, blindness, kidney problems and atherosclerosis. Some patients only using glucose lowering pills expressed the fear of nonadherence resulting in an increase of pharmacotherapy [Quote \#15].

\section{Motivational beliefs}

Near all HCPs and patients identified advantages of regular PA. In patients' perspective, frequently reported advantages were: feeling fitter, feeling more comfortable and energized, enjoying PA, and PA being good for their weight and glucose. Lowering their insulin use, decreasing the change of complications, and enjoying the social component of PA, were less frequently mentioned advantages. Near all patients and HCPs stated PA has no major disadvantages [Quote\#16]. Almost all patients identified advantages of a healthy diet. Having favorable blood glucose levels, feeling better, losing weight, and potentially decreasing their pharmacotherapy were most frequently mentioned advantages. Near all patients stated that a healthy diet doesn't involve disadvantages. Regarding adherence to pharmacotherapy, almost all patients reported its blood glucose lowering function as an advantage. Feeling healthier and believing pharmacotherapy controls T2DM, were other frequently mentioned advantageous beliefs. Most HCPs and patients indicated side effects, (fear of) hypoglycemia, feeling hungry, injection sites, weight gain, antipathy against introducing chemicals into their body, and social undesirability as disadvantages of pharmacotherapy adherence [Quote \#17]. 
Both HCPs and patients identified situations in which patients were perceived to have lower self-efficacy to adhere to their treatment strategies. Regarding PA adherence, lacking motivation, being busy, and feeling tired were most often mentioned situations. Less often mentioned situations were: if the weather is bad, when feeling ill or having to be physically active alone [Quote \#18]. Adherence to healthy diets is believed to be more difficult when: feeling tempted to eat unhealthy, feeling stresses, emotional or bored, having a party, and in weekends or evenings. These situations seem to provoke unhealthy snacking [Quote \#19]. Situations identified which might impede pharmacotherapy adherence were: being stressed, having a party, going out for diner, going on vacation, feeling ill, having an unstructured day/ life, or in the evening. A single time, patients identified situations like: not feeling burdened by T2DM and their partner not alerting them as difficult situations [Quotes \#20 and \#21].

Table 3 Interviewee quotes

\begin{tabular}{|c|c|c|}
\hline Quote number & Respondent & Quote \\
\hline \#1 & DN1 & $\begin{array}{l}\text { "If you don't plan it well concerning self-management, the } \\
\text { consultation, there is a disproportion... there are maybe } 10 \\
\text { minutes to talk about self-management... that is too short" }\end{array}$ \\
\hline \#2 & INT1 & $\begin{array}{l}\text { "Some do [prepare], the majority doesn't. I think it accounts for } \\
\text { most of our patients... they just sit down and wait and see what } \\
\text { happens" }\end{array}$ \\
\hline \#3 & T2DM3 & "They said: well, your sugar [level] should be better" \\
\hline \#4 & DN1 & $\begin{array}{l}\text { "Always small goals, very small steps, and also [to] approach it } \\
\text { positively. You feel that works for people. If they would just not } \\
\text { gain weight; that is also ok" }\end{array}$ \\
\hline \#5 & PN1 & $\begin{array}{l}\text { "If I notice that they want to set too high goals of which I know } \\
\text { they will never succeed in... than I will definitely say to them: well } \\
\text { very good that you thought about it, but maybe we should take } \\
\text { smaller steps" }\end{array}$ \\
\hline \#6 & DN1 & $\begin{array}{l}\text { "You are a kind of team that tries to help each other; the patient } \\
\text { helps you by giving information about their situation, and you try } \\
\text { to help the patient to gain control over their diabetes" }\end{array}$ \\
\hline \#7 & DIET2 & $\begin{array}{l}\text { "You often see in elderly, they say:"tell me what to do, what I } \\
\text { should inject, what I should eat..." following [advice] is not self- } \\
\text { management" }\end{array}$ \\
\hline \#8 & GP1 & $\begin{array}{l}\text { "They know they should be physically active for } 30-45 \text { minutes } \\
\text { daily, they know it, but they don't do it" }\end{array}$ \\
\hline \#9 & DN2 & $\begin{array}{l}\text { "A cookie is a habit rather than exception, and the food is not } \\
\text { limited to three meals daily" }\end{array}$ \\
\hline \#10 & T2DM11 & $\begin{array}{l}\text { "When it is } 3 \text { or } 4 \text { PM, I want to eat two cookies along with my } \\
\text { coffee, or some licorices, and in the evening... Once I start eating } \\
\text { a couple of sweets, than those taste buds... they keep asking for } \\
\text { sugar" }\end{array}$ \\
\hline
\end{tabular}


Table 3 Interviewee quotes

\section{Quote number Respondent Quote}

\begin{tabular}{|c|c|c|}
\hline \#11 & DN2 & $\begin{array}{l}\text { "I think at least 30\% doesn't always take that what they think } \\
\text { they should do, definitely [there] are people who forget a pill, } \\
\text { [and there] are always people who purposely don't take their } \\
\text { medication" }\end{array}$ \\
\hline$\# 12$ & T2DM8 & $\begin{array}{l}\text { "In particular with insulin, kind of cheating we call it, by injecting } \\
\text { a little more or a little less. Sometimes it works well, sometimes it } \\
\text { goes awfully wrong" }\end{array}$ \\
\hline$\# 13$ & T2DM7 & $\begin{array}{l}\text { "I think I will gain some weight... by expending more energy by } \\
\text { being physically active, I can use less insulin, which makes me } \\
\text { lose weight" }\end{array}$ \\
\hline \#14 & INT1 & $\begin{array}{l}\text { "You try to motivate them by pointing at the complications... } \\
\text { that's the hardest part of diabetes, you don't give medication } \\
\text { because people suffer from complaints, unless they have } \\
\text { hyperglycemia, but the approach is to invest in the future, to } \\
\text { prevent a heart attack, blindness and dialysis" }\end{array}$ \\
\hline \#15 & T2DM3 & $\begin{array}{l}\text { "I get tired and off course a lot can happen to your blood vessels, } \\
\text { [and] your eyes" }\end{array}$ \\
\hline \#16 & T2DM3 & $\begin{array}{l}\text { "I think [you] become more physically fit, and your sugar [level] } \\
\text { gets better... and my weight will decrease" }\end{array}$ \\
\hline \#17 & T2DM7 & $\begin{array}{l}\text { "I had abdominal pains, or [I was] nauseous of those pills, because } \\
\text { I had to take a lot of them" [T2DM7] }\end{array}$ \\
\hline$\# 18$ & GP1 & $\begin{array}{l}\text { "It can have various causes, that they don't understand it, or } \\
\text { because they have no money, or they don't have time, or because } \\
\text { it's really busy at home, or because they feel ashamed, many } \\
\text { different causes, or because they just don't feel like it" }\end{array}$ \\
\hline \#19 & DN2 & $\begin{array}{l}\text { "In between meals, and in the evening it happens even more, } \\
\text { because mostly they had dinner and it's 7:30 PM, and then of } \\
\text { course the closet opens and people sit in front of the television... } \\
\text { instead of doing some PA or walking, [they] snack, sometimes till } \\
\text { late in the evening" }\end{array}$ \\
\hline$\# 20$ & T2DM4 & $\begin{array}{l}\text { "Everything involving distraction creates the chance to forget } \\
\text { your pill" }\end{array}$ \\
\hline \#21 & DIET2 & $\begin{array}{l}\text { "If you have a normal day, you'll take your pills. But if a day is } \\
\text { really hectic... or they go out for dinner... then those pills are } \\
\text { forgotten" }\end{array}$ \\
\hline
\end{tabular}




\section{Discussion and conclusion}

\section{Discussion}

The first aim of this study was to explore if and how treatment adherence success factors are applied in consultations between HCPs and patients. In our population, diabetes consultations usually took place quarterly, were highly protocolled, and included discussing treatment adherence. This is in line with current Dutch guidelines (14). In line with HCPs' perceptions, most patients indicated not to prepare their consultations. Although HCPs pleaded for a more active contribution by their patients, few actually stimulated their patients to do so. HCPs and patients believed that decision making regarding consultation content and the formulation of treatment strategies was overall taking place in conjunction. However, a tendency towards reliance on HCPs' guidance was reported by HCPs as many patients, especially older patients, seemed to passively agree on HCPs' treatment suggestions. It is known that HCPs experience difficulties in involving patients in decision making (63). Previous research showed that patients should be active collaborators in care, that HCPs should enable patients to make informed decisions, and that treatment decisions should preferably be made in conjunction $(104,114)$. Moreover, SDM should be at the center of diabetes consultations according to Dutch guidelines (14). Applying SDM principles not only has the potential to improve quality of life, it has also been shown to improve physiological markers such as HbA1c-levels (115). Although both HCPs and patients indicated that patients should be primarily responsible for treatment adherence, our results suggest that the HCP is the main initiator and responsible for making treatment decisions. This can have considerable unfavourable consequences for the execution of those strategies (115).

Contrary to perspectives of HCPs, most patients perceived that during consultations, no or not concrete enough treatment goals were set in order to manage their diabetes. Such perspective discrepancies between HCPs and patients might lead to not reaching treatment goals. As a consequence, nurses sometimes feel powerless if their patients do no reach treatment goals (63). Earlier, the importance of applying goal setting principles in achieving behaviour change has been emphasised (116), and this approach has been shown to result in significant improvements in HbA1c-levels (117). Although according to HCPs goal setting was applied, active patient engagement, clarity about goal setting, and adequate self-management education on how to achieve those goals, seemed to be lacking. HCPs perceived that patients sometimes aimed for higher, unachievable goals. As a reaction, they indicated to tailor strategies to patients' abilities and proposed taking small steps in behaviour change. Despite these efforts of HCPs, it is known that many HCPs aren't trained to aid patients in setting achievable goals, and that they might lack knowledge in facilitating patients to set small goals or to make action plans (116). In addition, HCPs might be willing to set goals for their patients themselves. While most HCPs and patients indicated to make treatment plans in conjunction, it seemed that especially older patients could be characterised as rather passive collaborators. Contrary to younger and more active patients, older patients should not only feel responsible 
for the execution of treatment strategies, but should also be actively involved in setting them. This leads to increased motivation and self-efficacy, and has positive consequences on its execution $(106,115,118)$. This is in particular of interest because HCPs reported that one reason for not reaching treatment goals in patients was a lack of motivation.

The second aim was to explore salient personal beliefs about T2DM treatment, including both healthy lifestyle adaptations and pharmacotherapy. First, this study indicated that adherence to the core diabetes treatment elements was suboptimal. HCPs emphasized that adherence was poor regarding all treatment elements, contrary to some patients who stated to adhere to one or more treatment elements. Both HCPs and patients felt that knowledge regarding PA was sufficient, however regarding knowledge of healthy diets, their perspectives differed: HCPs thought that this inadequate while patients thought that this was adequate. Regarding pharmacotherapy, both groups reported that patients' knowledge was insufficient. Many patients were aware of the susceptibility of getting complications as a result of non-adherence to core treatment elements, although most of these were short-term consequences. It is notable that despite the reported poor adherence, many advantageous beliefs were reported of adherence to all treatment elements. Contrary to PA and healthy diets, pharmacotherapy adherence also yielded many disadvantages. Larkin et al. (119) reported that in particular non-adherent patients were more likely to be worried about pharmacological side effects. Disadvantages like these are frequently reported regarding taking oral medication (120), should be thoroughly discussed in consultations, and patients should be made aware that the advantages of adherence outweigh the disadvantages (106). Last, self-efficacy to adhere to treatment strategies seemed to be lower in certain difficult situations, which tend to occur when daily routine is compromised, like having an unstructured day/life. Similar difficult situations were reported for PA, healthy diets and pharmacotherapy. Low self-efficacy has been related to reduced adherence levels earlier (121), and should therefore be enhanced, especially in these situations out of daily routine.

This study shows some strengths and limitations. First, HCPs and patients were interviewed, allowing comparison of both perspectives. Second, a theoretical framework (The I-Change Model) was used which incorporates factors that are known to determine behaviors involved in diabetes treatment. These factors were operationalized to contribute to a better understanding of the underlying salient beliefs. Last, our data was analyzed systematically using a deductive coding approach with a pre-defined coding tree. Our analysis showed satisfying intercoder reliability and agreement levels. This study also has some limitations. First, participants were recruited from one geographical region. Recruitment of HCPs proved to be more difficult than expected as just one internist was recruited. Despite efforts to create sample heterogeneity, future studies should aim for nation-wide recruitment to increase generalizability. Second, some interviews were split in two parts because of patients' time restraints. Future interviews should possibly be more concise and set time limits. Third, post-motivational determinants like action planning and coping planning should also receive attention in terms of identifying 
salient personal beliefs regarding these determinants. Last, the qualitative design of the study yielded in-depth findings, but observational research designs are required to objectively assess our findings.

\section{Conclusion}

Our T2DM patient sample shows insufficient metabolic control and both perspectives of HCPs and patients add up to the conclusion that both lifestyle and medication adherence is suboptimal. Most patients visited their consultations unprepared, justified by perspectives of both interviewed groups. Patients did not or only vaguely experience that treatment goals were set, whereas HCPs indicated to actually provide them. Although both groups believed that SDM is applied, it seemed a rather passive collaboration, with the HCP mostly in charge of making treatment decisions. However, contrary to older patients, younger patients could actually be characterised as more active collaborators. A lack of motivation and inactive involvement of patients might justify poor treatment adherence. Also the inability of HCPs to involve patients in decision making, the potential incapacity of HCPs to set achievable goals for their patients, and the misconception between HCPs and patients about if goals are actually set, could contribute to poor treatment adherence. Regarding personal beliefs, awareness factors seemed to be sufficient. However, perspectives regarding healthy nutrition knowledge of patients, differed between HCPs and patients, and pharmacotherapy knowledge and awareness of long-term consequences could be improved. Despite reporting many advantages of treatment adherence, and - excluding pharmacotherapy - few disadvantages, treatment adherence was still suboptimal. As indicated by HCPs and patients, self-efficacy seemed to be lower in situations outside daily routine, and should be enhanced to improve treatment adherence.

\section{Practice Implications}

A fruitful collaboration between HCPs and T2DM patients should build on input and active engagement from both parties. Shared-agreement and clarity about goals, taking small steps in behaviour change, and tailoring goals to patients' needs and abilities are considered key factors in facilitating treatment adherence. HCPs should make patients aware that they should also be responsible for active preparation, engagement and collaboration in the formation of treatment strategies. Self-management education, accompanied by use of salient personal beliefs about T2DM treatment, should be cornerstone of consultations. These can enhance the necessary motivation for improving treatment adherence, support adequate selfmanagement, and facilitate persisted behaviour change outside consultation time. 

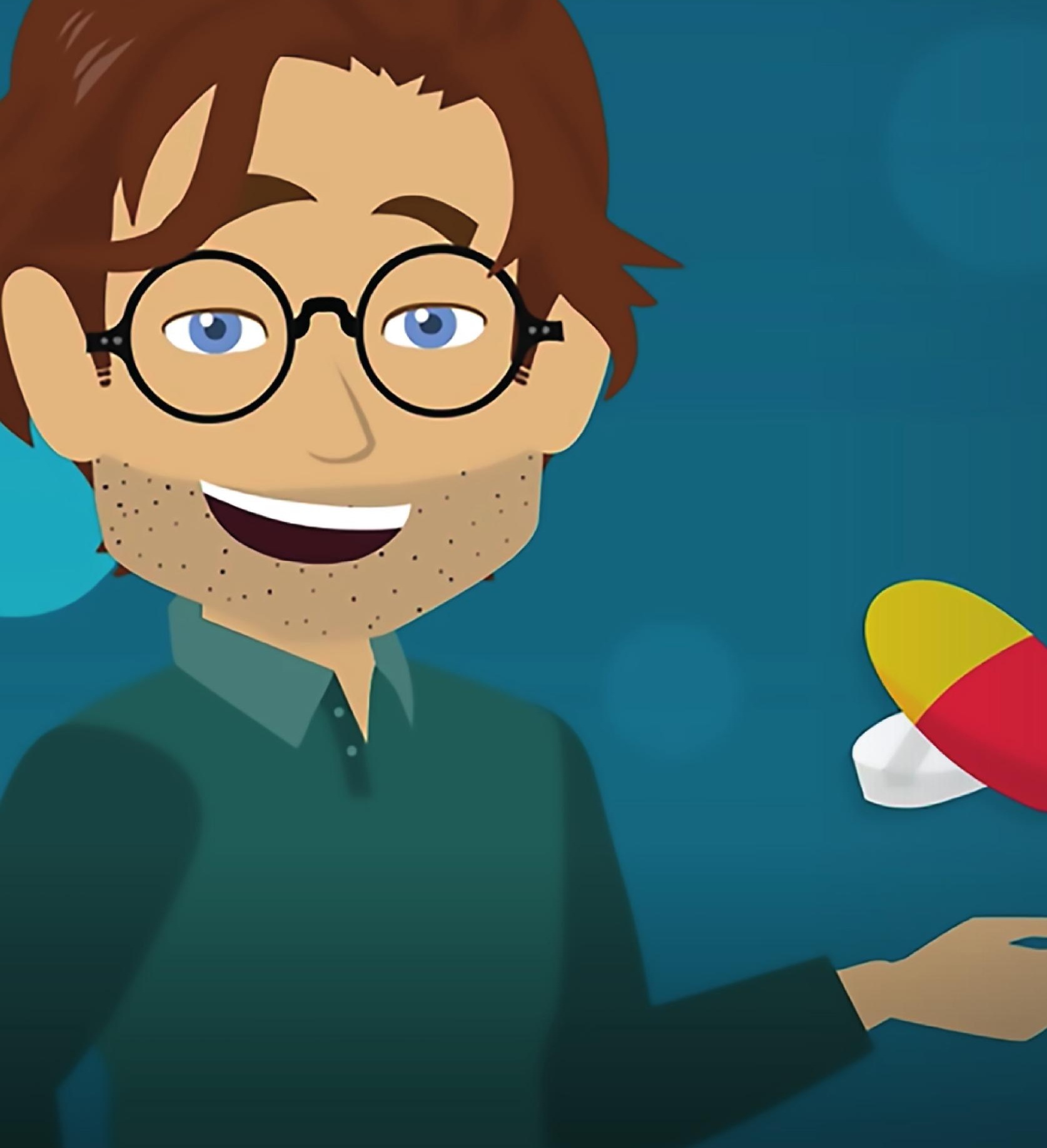


\section{Chapter 3}

\section{Psychological predictors of adherence to oral hypoglycaemic agents: An application of the ProMAS questionnaire}

\section{This chapter has been published as:}

Vluggen S, Hoving C, Schaper NC, de Vries H. Psychological predictors of adherence to oral hypoglycaemic agents: An application of the ProMAS questionnaire. 


\section{Abstract}

Objectives: This study aimed to identify psychological predictors of oral hypoglycaemic agent $(\mathrm{OHA})$ adherence and to compare adherence rates between a novel and well-known instrument, i.e. the ProMAS and MARS-5.

Design and main outcome measures: A longitudinal study design was applied with surveys at baseline and six-months follow-up. At baseline, OHA adherence using the ProMAS and MARS-5, socio-cognitive determinants, and demographics were assessed. At follow-up, the ProMAS was applied as outcome measure, on which socio-cognitive determinants and demographics were regressed using linear regression analysis.

Results: The baseline and follow-up sample included 304 and 231 participants respectively. When applying cut-off points of $\geq 15$ for the ProMAS and $\geq 23$ for the MARS-5, $47.7 \%$ and $89.5 \%$ adhered to their OHAs. Consistent predictors of better adherence comprised a low education, lower severity perceptions, and higher self-efficacy and intention. After correcting for baseline adherence, a low education and higher self-efficacy remained significant adherence predictors.

Conclusions: Compared to the MARS-5, ProMAS data was less skewed, similar to objectively collected data, and yielded insights in a broader spectrum of (non)-adherence behaviours. Results stress the need for adherence improving interventions which particularly should target higher educated patients and patients with low self-efficacy, low intention and high severity perceptions. 


\section{Introduction}

The long-term beneficial effects of intensive glycemic control in type 2 diabetes patients on disease progression, cardiovascular risks, and early mortality are well known (16). Initial treatment strategies comprise healthy lifestyle recommendations (30), i.e. improving dietary patterns, regular physical activity, smoking cessation, moderate alcohol consumption, and a healthy Body Mass Index (BMI) (25). Only 3.5\% of the 40-75 year old patients achieves adherence to all these recommendations which often makes the initiation of pharmaceutical interventions inevitable (25). Depending on the necessity of treatment intensity, oral hypoglycaemic agents (OHAs) are usually considered primary pharmaceutical strategies. As insulin secretory capacities of the pancreas progressively decrease, eventually many patients will require more than one drug, whether or not combined with insulin therapy.

Despite the pivotal role of $\mathrm{OHAs}$ in pursuing glycemic control, adherence rates are suboptimal (30). Suboptimal adherence not only attenuates beneficial treatment outcomes (16), but is also associated with reduced quality of life, increased healthcare costs and hospitalizations, and early mortality (18). Adherence rates range widely across studies between 38.5-93.1\% which is probably largely attributable to the variety of objective and subjective measurement instruments used (30). Subjective measurement instruments, i.e. self-report questionnaires, are most often employed, given their low costs, usability, potential to test large sample sizes, and the wide range of adherence behaviours that can be assessed (122-124). The Medication Adherence Report Scale (MARS-5) (125) and the Morisky Medication adherence Scale (MMAS) (126) are frequently used self-report instruments that have been developed for adherence research involving chronic illnesses $(122,127)$. These validated questionnaires have demonstrated sufficient internal reliability (125), but faced criticism regarding their often highly skewed adherence distributions and limited range of adherence behaviours assessed $(128,129)$. Therefore, new instruments are needed that assess adherence behaviour more accurately.

The Probabilistic Medication Adherence Scale (ProMAS) (124) has recently been developed in response to flaws in existing self-report instruments and could potentially solve these measurement issues. The ProMAS assesses eighteen adherence behaviours with varying item difficulty, selected from extensive literature review and patient interviews (124). Hitherto, research showed that the ProMAS, when compared to the MARS-5, yields less skewed adherence distributions, shows a better match with data collected by objective methods (33), and provides in depth insights in which adherence behaviours are improvable (124). However, additional research is needed to confirm findings, particularly to assess the applicability of the ProMAS for specific disease states such as type 2 diabetes.

As OHA adherence can vary markedly between individuals, not only new instruments that measure adherence more accurately are needed, but preferably these instruments should 
also identify factors which predict adherence. This in order to develop effective adherence improving interventions. Reviews conclude that adherence is a complex process in which various domains of adherence determinants interact, i.e. social and economic factors, healthcare system factors, therapy-related factors, condition-related factors, and patientrelated factors (33). Although all domains may provide relevant intervention targets to improve adherence, most of these domains are difficult to change and might only influence adherence indirectly through perceptions of the patient (33). Hence, patient-related factors, including socio-cognitive determinants such as a person's awareness, motivation, intention and self-regulation (43), seem to be the most viable domain to intervene in. Nonetheless, only few studies have applied socio-cognitive theoretical models to explain $\mathrm{OHA}$ adherence behaviour $(130,131)$ and only one was quantitative in nature. This cross-sectional study showed negative associations of perceived general barriers and perceived side-effect barriers, and a positive association of self-efficacy with medication adherence as measured with the MMAS (130). To the best of our knowledge, studies have not yet applied longitudinal designs, allowing the identification of factors with predictive value for $\mathrm{OHA}$ adherence, and have not yet applied promising adherence assessing instruments such as the ProMAS. Therefore, the aim of the current study was twofold.

The first aim was to compare OHA adherence between the ProMAS and MARS-5. The second aim was to identify socio-cognitive predictors of OHA adherence using the ProMAS as outcome.

\section{Materials and methods}

A longitudinal study design was applied in type 2 diabetes patients with surveys at baseline and six-months follow-up. The study was approved by the Medical Ethical Committees of the Maastricht University Medical Center (15-4-181) and Zuyderland Hospital (15-N-209).

\section{Participants and procedure}

The current study is part of a larger project which also aims to identify socio-cognitive predictors of adherence to insulin therapy. Two hospitals with outpatient clinics and 55 general practices in the southern part of the Netherlands were approached to aid in recruiting participants. All hospitals and six practices indicated their willingness to participate. Patients were eligible if diagnosed with type 2 diabetes, aged 40-70 years old, and used at least one type of blood glucose lowering medication, i.e. either OHAs and/or insulin therapy. Patients not able to speak, understand or write the Dutch language were excluded. A total of 1674 potential participants were invited by the research team to participate via postal mail by an information letter (personalised with a signature from their own physician), an informed consent form and the baseline questionnaire. In case participants completed and returned the baseline questionnaire, they were sent the follow-up questionnaire six months afterwards. 
For the purpose of the current study, we aimed to retain 182 participants at follow-up to ensure enough study power, taking into account an a of 0.05 and a study power of 0.8 (132).

\section{Questionnaires}

The baseline questionnaire consisted of 130 questions and the follow-up questionnaire consisted of 78 questions on adherence to OHAs, socio-cognitive determinants, and demographics.

\section{Adherence to OHAs}

Adherence to OHAs was assessed by the ProMAS (124) and MARS-5 (127). The ProMAS was assessed at baseline and follow-up, the MARS-5 at baseline. Both instruments assess adherence without a time frame over which adherence is assessed. This implies that adherence is assessed from medication initiation onwards which in most diabetes patients can add up to several decades. This might not only lead to a questionable relevance of results, but also to results which are subject to recall-bias due to a not recent enough recall time-frame (122). Moreover, in the Netherlands, most patients visit their physician quarterly for a diabetes check-up in which guidelines recommend that therapy adherence should be one of the main discussion topics (14). Based on these arguments, adding a three-month time-frame over which adherence behaviours were assessed to each item of the ProMAS and MARS-5, was considered tenable, e.g. 'it has happened at least once in the last three months that I forgot to take (one of) my oral hypoglycaemic agents.' The eighteen items of the ProMAS can be scored on a binary answering scale ( $1=y e s$, true or $0=$ no, not true) and assess various adherence behaviours, e.g. forgetting medication, taking less or more medication, and changing medication dosages. A higher sum score represents better adherence (range 0 -18). The outcome can be applied either as continuous or dichotomised measure in which a score of $\geq 15$ is considered high adherence (124).

The MARS- 5 consists of five items which can be scored on a 5-point scale ( 1 =always $-5=$ never) and assesses adherence behaviours, e.g. forgetting, stopping, and skipping medication (127). Its sum score ranges from 5-25, with higher scores representing better adherence, and is most often dichotomised to distinguish between non-adherence and adherence. Although no golden standard exists, a cut-off point at $\geq 23$ is widely applied and considered high adherence (133).

\section{Socio-cognitive determinants}

Socio-cognitive determinants were derived from the I-Change Model (ICM) (43), which is a theoretical framework integrating various well-known socio-cognitive theories $(36,37,109)$. The ICM (figure 1) has been applied frequently to map salient beliefs of health behaviour (change), including medication adherence (134). The model assumes that behaviour can partly be explained by socio-cognitive determinants and that behaviour change is a phased process. The ICM distinguishes between three phases; an awareness, motivation and action 
phase, influenced by information and preceding factors. Awareness determinants are believed to have a distal indirect influence on behaviour and include a person's knowledge, risk perceptions, i.e. how susceptible someone feels to get a certain illness and how severe this illness is valued, and salient cues to action, i.e. prompts which trigger engagement in health behaviour. Subsequently, these awareness determinants influence distinct motivational determinants such as attitudes towards performing health behaviour (pros and cons), perceptions of social influences (support, modeling and norms), and self-efficacy, i.e. a person's perception of their own competence to successfully execute a health behaviour in difficult situations. A person's intention to change health behaviour is influenced by these motivational determinants. However, a high intention towards behaviour change does not consistently warrant successful behaviour change (56). Action phase determinants facilitate this process by the formation of preparatory plans and coping plans, which are assumed to increase the likelihood of successful translation of expressed intentions into the pursued behaviour. Distinct determinants were assessed through several salient beliefs, derived from earlier research (28), and combined into scales.

Figure 1 The l-Change Model as applied in this study

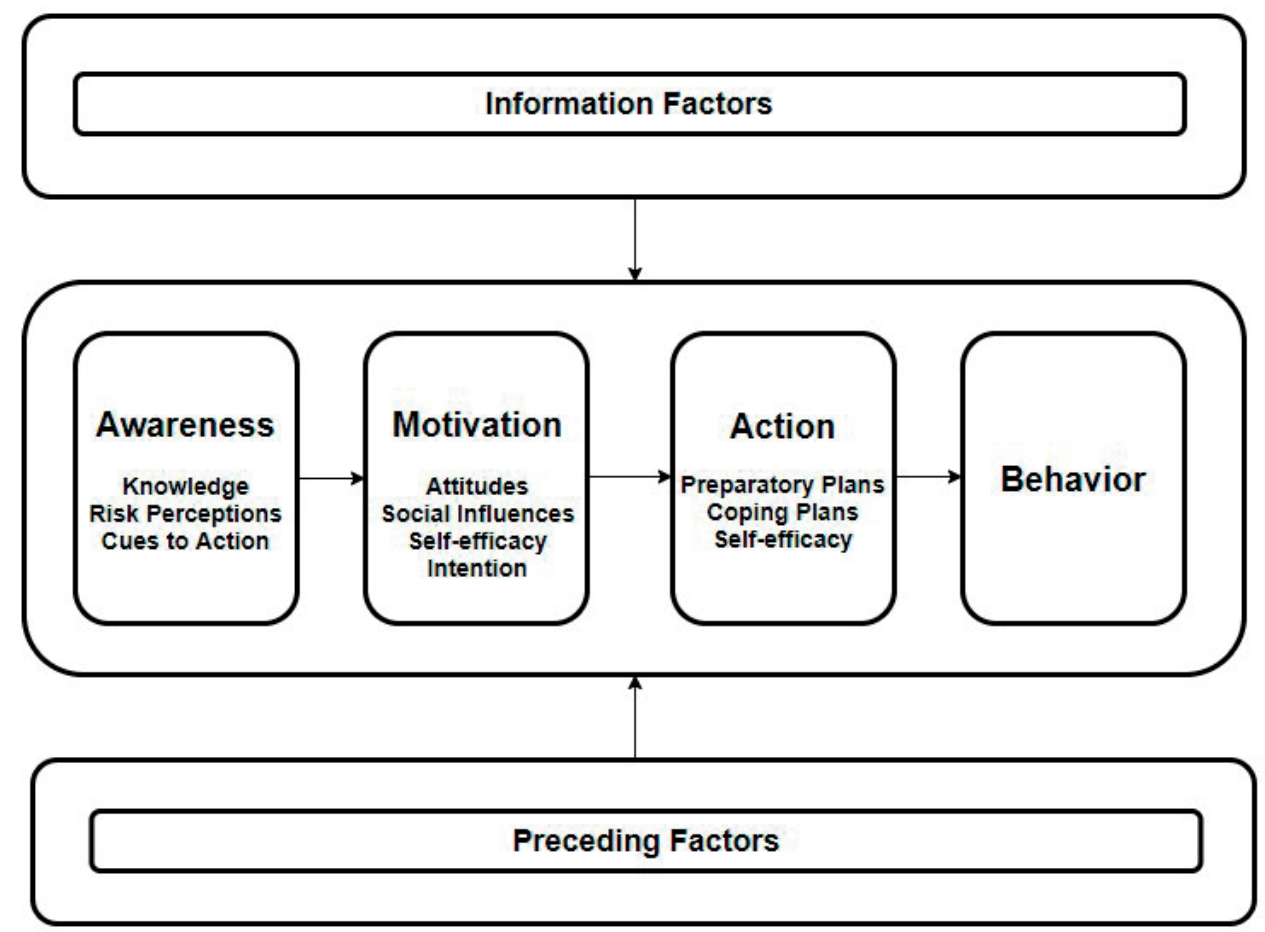


Knowledge was assessed by ten items, e.g. shaking and sweating are signs of a too high blood glucose level, which could be scored on a binary answering scale ( $1=$ true or $0=$ false). An overall sum score was calculated ranging from $0-10$, with higher scores representing more knowledge.

Risk perceptions were assessed with eight items each for perceived susceptibility ( $a=0.83$ ), e.g. susceptibility of developing visual conditions in the future ( $1=$ very unlikely $-5=$ very likely) and perceived severity $(a=0.88)$, e.g. severity of having a heart attack in the future is $(1=$ not serious at all $-5=$ very serious).

Cues to action ( $\alpha=0.94$ ) were measured by eleven items, e.g. when I read information about $\mathrm{OHAs}$, I am prompted to use my OHAs as prescribed (1=totally disagree $-5=$ totally agree).

Attitudes were measured using six items each for perceived pros ( $\mathrm{a}=0.87$ ), e.g. when I use my OHAs as prescribed, I ( $1=$ do not feel healthier $-4=$ feel much healthier), and perceived cons ( $a=0.70)$, e.g. when I use my OHAs as prescribed, I ( $1=$ suffer a lot of side effects $-4=$ do not suffer side effects).

Perceived social influences were assessed with eleven items including support ( 4 items, $a=0.80$ ), e.g. my partner supports me to use my OHAs as prescribed, modeling ( 3 items, $a=0.78$ ), e.g. my family members use their medicines as prescribed, and norms ( 4 items, $a=0.82$ ), e.g. my physician thinks I should use my OHAs as prescribed ( 1 =totally disagree $-5=$ totally agree).

Self-efficacy was measured with eleven items, representing eleven salient out of routine situation in which self-efficacy to be adherent might be reduced ( $a=0.89$ ), e.g. how difficult or easy is it for you to use your OHAs as prescribed when being on vacation (1=very difficult $-5=$ very easy).

Intention was assessed with two items $(\alpha=0.90)$, i.e. I plan/I want to use my OHAs as prescribed (1=totally disagree $-5=$ totally agree).

Preparatory plans $(a=0.77)$, e.g. I plan to put my OHAs in a fixed place, and coping plans ( $a=0.98$ ), e.g. I have a plan to use my OHAs in a difficult situation such as being on vacation, were assessed with seven and eleven items respectively ( $1=$ totally disagree $-5=$ totally agree).

\section{Demographics and dossier data}

Demographic characteristics included gender ( $1=$ male, $2=$ female), age, education level (1=low, 2=medium, 3=high), relationship status (1=alone, 2=together with partner), nationality, length, weight, consultation content and a description of prescribed medication. From the participants' electronic patient dossier data were extracted on systolic and diastolic 
blood pressure, as well as the laboratory values of $\mathrm{HbA}_{1 c}$-level, creatinine clearance, and LDLcholesterol. Body Mass Index (BMI) was calculated as weight/length².

\section{Statistical analyses}

Analyses were performed using SPSS 24.0, applying a significance level of 0.05. Missing data was imputed applying principles of Downey and colleague (135). Participants missing over $20 \%$ of the total data or participants missing data on outcome measure(s) were excluded from the analysis.

Descriptive statistics and frequencies were applied to describe sample characteristics. A logistic regression was performed regarding demographic variables, i.e. gender, age, education level and relationship status, to identify potential selective attrition between baseline and follow-up.

To answer the first aim, descriptive statistics and frequencies were applied to map both overall adherence scores of the baseline ProMAS and MARS-5 as well as scores on individual questionnaire items. Overall adherence scores were compared by applying dichotomised outcome measures. Subsequently, bivariate analyses between demographics, socio-cognitive determinants, and follow-up adherence (ProMAS) were performed using Pearson's correlations. Additionally, a correlational analysis was performed between the continuous baseline outcome of the ProMAS and MARS-5. To answer the second aim, multiple linear regression analysis was performed by regressing demographics, socio-cognitive determinants and the baseline ProMAS sum score on the continuous ProMAS follow-up adherence sum score. The selection of variables to include in the multiple regression analysis was based on the assumption of the theoretical framework that was applied in the study (136). The model assumes that sociocognitive variables (e.g. knowledge and self-efficacy), patient background variables (e.g. age, gender, and education level), and clinical outcomes (e.g. $\mathrm{HbA}_{1 c}$-level) may either directly or indirectly influence adherence behaviour (43). Independent predictor variables were entered hierarchically in the analysis according to the assumptions of the ICM: 1) demographics and dossier data, 2) awareness determinants, 3) motivational determinants and intention, 4) action determinants, and 5) baseline behaviour. Baseline behaviour was added in the last regression model to examine its impact on socio-cognitive predictors entered in previous models.

\section{Results}

\section{Participants}

The flow of participants throughout the study is shown figure 2. Five hundred participants returned the baseline questionnaire (29.9\% response rate). Of the 362 participants included in the baseline sample, 260 returned the follow-up questionnaire (72\% response rate). After exclusion of participants missing over $20 \%$ of the data or with missing data on the outcome 
measure(s) (135), data of 304 participants remained for the analysis of the first study aim, and data of 231 participants for the second study aim. As described in table 1, two third of population was male, the mean age was 60.8 years and the majority of participants lived together with a partner. Education level was fairly equally distributed. Participants had a relative long average disease duration of over 12 years. The majority of participants was obese with inadequate blood glucose control as reflected by the elevated mean $\mathrm{HbA}_{1 c}$-level, while the mean LDL-cholesterol was well within target range for many participants (14). Two thirds used one class of OHA medication; nearly $90 \%$ used metformin. Near half of the participants applied insulin therapy alongside their OHAs. The attrition analysis revealed that participants with a low education level were more likely to drop-out during follow-up (OR=1.85, $p=.02$ ).

Table 1 Sample characteristics

\begin{tabular}{lll}
\hline & N (\%) & Mean (SD) \\
\hline Age & & $60.8(6.8)$ \\
Gender & & \\
Male & $109(67)$ & \\
Female & & \\
Education level & $96(30.8)$ & \\
Low & $110(35.3)$ \\
Middle & $98(31.4)$ \\
High & \\
Relationship status & $237(76)$ \\
Together with partner & $74(23.7)$ \\
Alone & \\
Diabetes pharmacotherapy & $176(56.4)$ \\
OHA(s) only & $136(43.6)$ & \\
OHA(s) and insulin therapy & & \\
Number of different OHAs in use & $212(67.9)$ & \\
One & $67(21.5)$ & \\
Two & $14(4.5)$ & \\
Three & $273(87.5)$ & \\
OHA medication type & & \\
Metformin & & \\
Glimepiride & & \\
Gliclazide & & \\
\hline
\end{tabular}

Continue 
Table 1 Sample characteristics

N (\%)

Mean (SD)

Treatment sector

Primary care

Secondary care

ProMAS Baseline Score

MARS-5 Basline Score

ProMAS Follow-up Score

Diabetes duration (years)

$\mathrm{HbA}_{1 \mathrm{c}}$-level $(\mathrm{mmol} / \mathrm{mol})^{\mathrm{a}}$

BMI $\left(\mathrm{kg} / \mathrm{m}^{2}\right)$

Creatinin Clearance $(\mathrm{ml} / \mathrm{min})^{\mathrm{b}}$

LDL-Cholesterol (mmol/l)

Blood pressure $(\mathrm{mmHg})$

Systolic

Diastolic
$73(23.4)$

239 (76.6)
$13.6(3.6)$

$24.1(1.4)$

$13.8(3.4)$

$12.3(7.7)$

$61(12.7)$

$31.9(5.2)$

$88.1(24.2)$

$2.1(0.7)$

$133.6(14.1)$

$76.8(10.1)$

OHA oral hypoglycaemic agent(s), BMl body mass index, $\mathrm{HbA}_{1 c}$ glycosylated haemoglobin, SD standard deviation a This equals an $\mathrm{Hb}_{1 c}$-level of $7.7 \%$

${ }^{b}$ Estimation of glomerular filtration rate 
Figure 2 Flow of participants throughout study

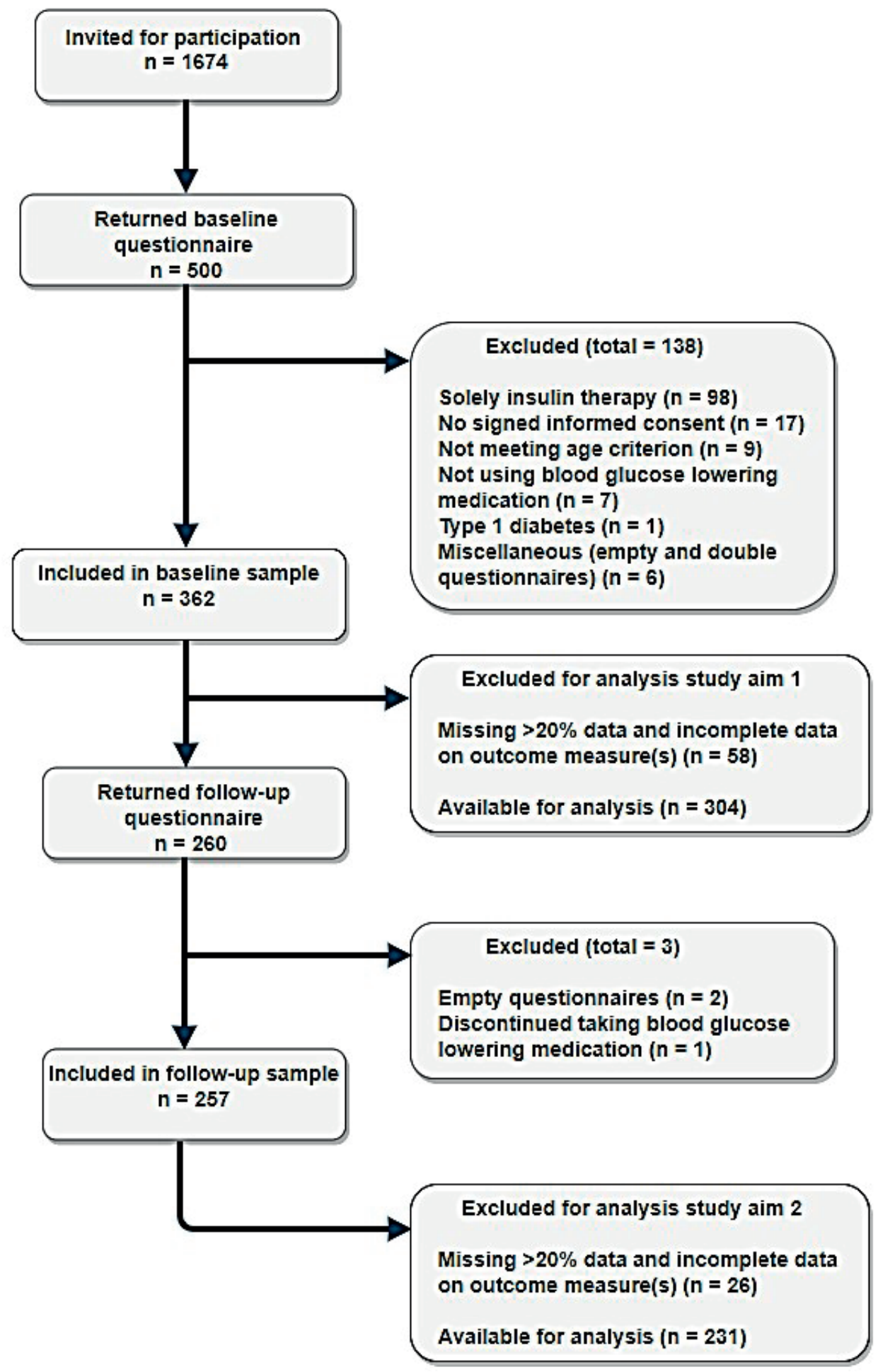




\section{Overall ProMAS and MARS-5 adherence scores}

The average baseline adherence score was 13.6 (range 2-18, SD = 3.6) for the ProMAS and 24.1 (range $16-25, S D=1.4$ ) for the MARS- 5 . With a cut-off point of $\geq 15,47.7 \%$ of the respondents adhered to their OHAs regarding the ProMAS, while $89.5 \%$ of the respondents achieved $\mathrm{OHA}$ adherence regarding the MARS- 5 when using a cut-off point of $\geq 23$.

\section{Adherence assessment scores}

Scoring percentages to items assessed in the ProMAS and MARS-5 are shown in table 2. Regarding the ProMAS, items two and six were reported as non-adherent by more than half of the participants, and both comprise adherence behaviours concerning medication timing. Items one, five and ten were reported as non-adherent by over $40 \%$ of the participants, and comprise adherence behaviours such as forgetting medication, being positive about having taken all the required medication, and having taken medication at a different moment than prescribed (e.g. with breakfast or in the evening). Items which comprised adherence behaviours such as (temporarily) stopping medication, changing medication dosages, filling prescriptions, initiating medication taking, and taking more medication than prescribed, were reported as non-adherent by less than five percent of participants.

In the MARS-5 questionnaire, slightly more than $50 \%$ of the participants reported to have never forgotten to take their OHAs, while nearly all others indicated that this occurred rarely or sometimes. Over $95 \%$ of the participants indicated to have never altered the dose of their OHAs, stopped taking their OHAs, or decided to skip one of their OHAs intakes. 


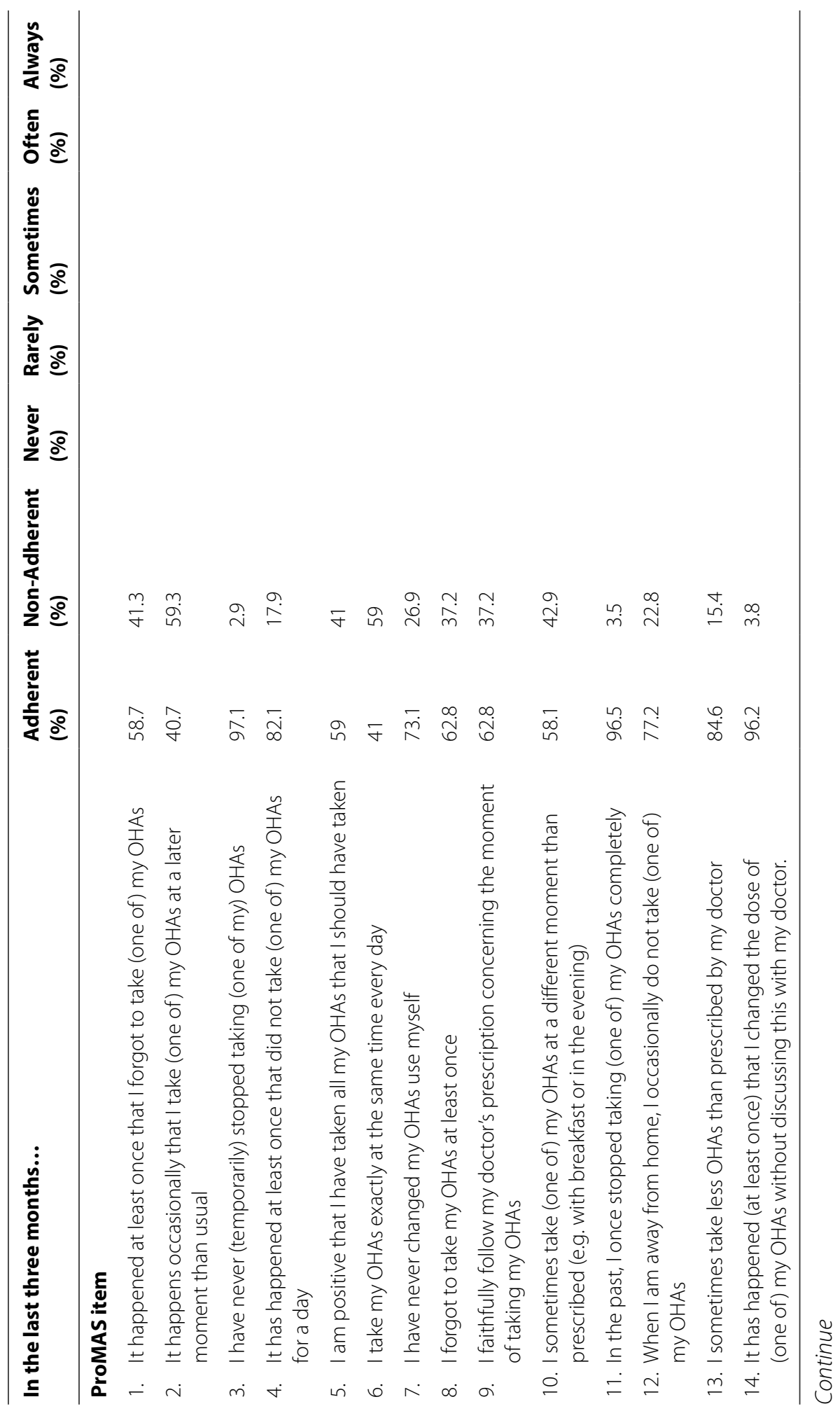




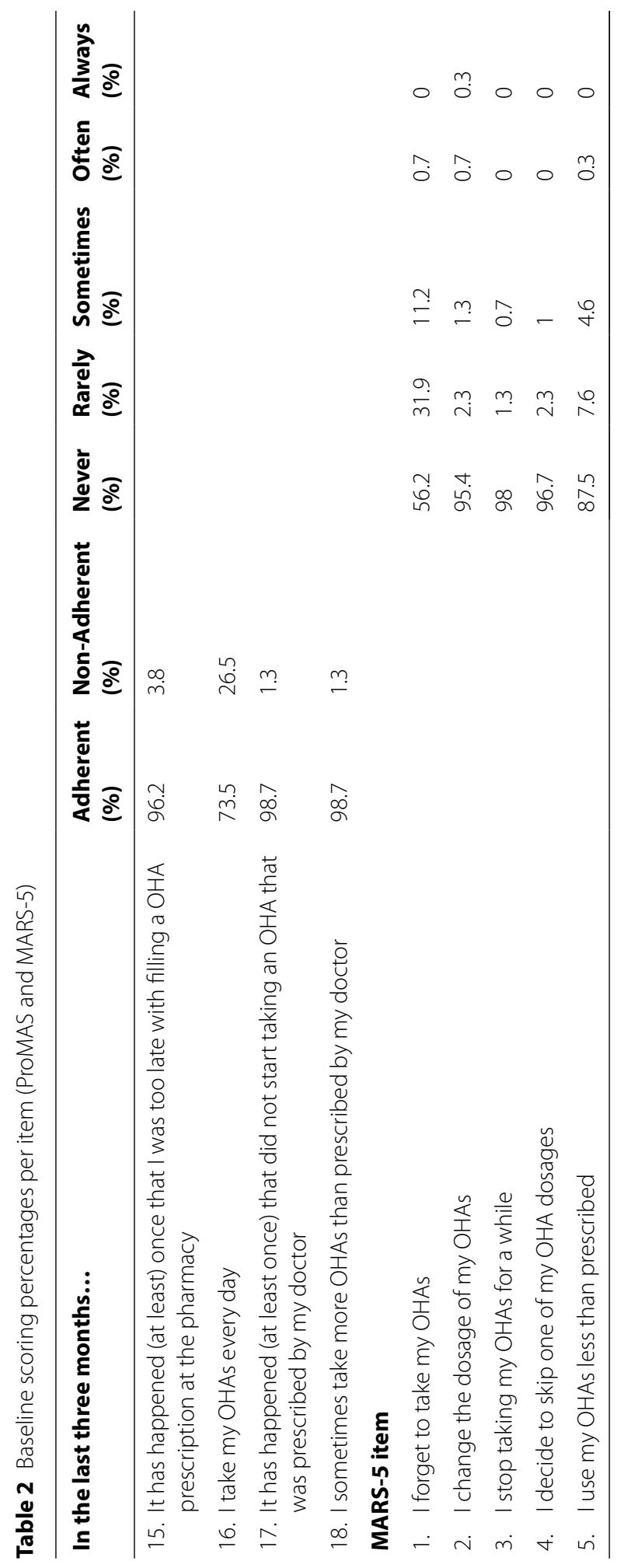




\section{Bivariate analyses}

\section{Correlations of demographics and socio-cognitions with follow-up adherence}

As shown in table 3, older participants, as well as participants perceiving more pros, having a higher self-efficacy and higher intention to adhere, showed higher levels of adherence. Moreover, participants who made a higher number of preparatory plans and coping plans for difficult situations, showed better adherence. Significant negative correlations with adherence indicated that participants with a high education level had lower adherence scores compared to low educated participants, and that participants with a lower $\mathrm{HbA}_{1 \mathrm{c}}$-level and lower risk perceptions showed better adherence. Participants perceiving more cues to action and more cons significantly showed lower levels of adherence.

\section{Correlations of outcome measures}

A moderate to large correlation was observed between the ProMAS and the MARS-5 ( $r=.67$; $p=.00$ ), representing a substantial positive association between both measures (137).

\section{Predictors of OHA adherence}

Results of the regression analysis are displayed in table 4. Results of model one demonstrate that higher age and a low education level predicted better adherence. In model two, participants with a high score on cues to action and severity perceptions were less adherent. Participants with a low education maintained showing better adherence. In model three, education level and severity perceptions remained significant, while cues to action became insignificant. Living together, a high self-efficacy and intention were other predictors of better adherence. In the fourth model, the significance of relationship status disappeared, while the other factors remained significant. In the fifth model, the significance of severity perceptions and intention disappeared, and a low education level, high self-efficacy and baseline adherence predicted better adherence at follow-up. The fifth model explained $48 \%$ of the total variance in $\mathrm{OHA}$ adherence. 


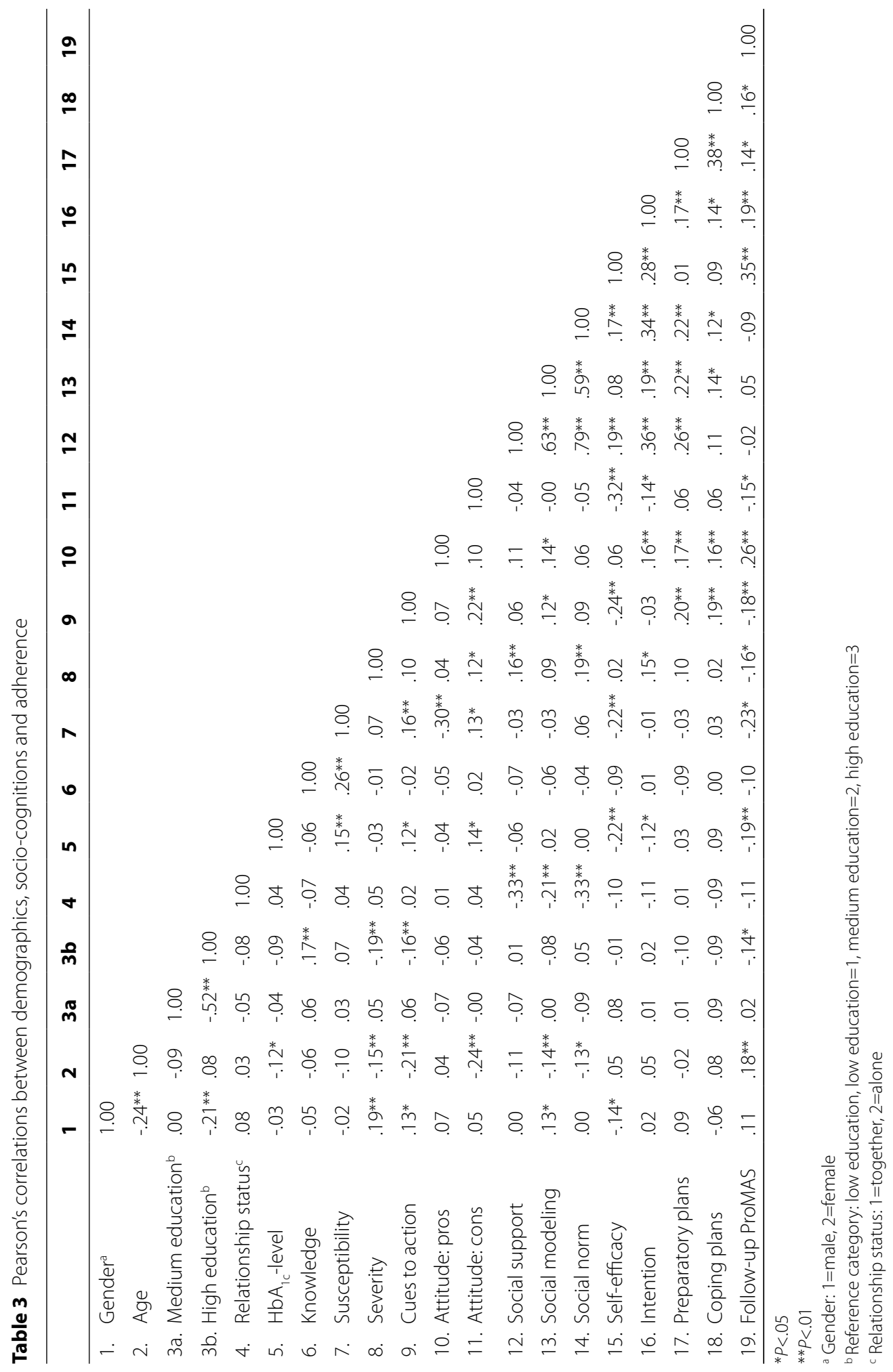


Table 4 Predictors of OHA adherence at six-months follow-up

\begin{tabular}{|c|c|c|c|c|c|}
\hline & $\begin{array}{l}\text { Model } 1 \\
\beta\end{array}$ & $\begin{array}{l}\text { Model } 2 \\
\beta\end{array}$ & $\begin{array}{l}\text { Model } 3 \\
\beta\end{array}$ & $\begin{array}{l}\text { Model } 4 \\
\beta\end{array}$ & $\begin{array}{l}\text { Model } 5 \\
\beta\end{array}$ \\
\hline Gender $^{a}$ & .11 & .14 & .11 & .14 & .04 \\
\hline Age & $.20^{* *}$ & .14 & 12 & .10 & .08 \\
\hline Medium education ${ }^{b}$ & -.09 & -.11 & -.13 & -.15 & -.08 \\
\hline High education ${ }^{b}$ & $-.23^{* *}$ & $-.28^{* *}$ & $-.23^{*}$ & $-.23^{*}$ & $-.17^{*}$ \\
\hline Relationship status ${ }^{c}$ & -.13 & -.13 & $-.14^{*}$ & -.13 & -.07 \\
\hline $\mathrm{HbA}_{1 c}$-level & -.13 & -.11 & -.04 & -.04 & -.01 \\
\hline Knowledge & & .01 & .04 & .04 & .03 \\
\hline Susceptibility & & -.10 & -.02 & -.02 & -.07 \\
\hline Severity & & $-.20^{* *}$ & $-.22^{* *}$ & $-.21^{* *}$ & -.12 \\
\hline Cues to action & & $-.15^{*}$ & -.07 & -.11 & -.09 \\
\hline Attitude: pros & & & .10 & .08 & .06 \\
\hline Attitude: cons & & & -.01 & -.02 & .01 \\
\hline Social support & & & -.08 & -.09 & -.11 \\
\hline Social modeling & & & .09 & .07 & .06 \\
\hline Social norm & & & -.09 & -.10 & -.01 \\
\hline Self-efficacy & & & $.26^{* *}$ & $.25^{* *}$ & $.16^{*}$ \\
\hline Intention & & & $.22^{* *}$ & $.19^{*}$ & .10 \\
\hline Preparatory plans & & & & .07 & .02 \\
\hline Coping plans & & & & .11 & .09 \\
\hline Baseline $\mathrm{OHA}$ adherence & & & & & $.45^{* *}$ \\
\hline$R^{2}$ & .12 & .20 & .32 & .34 & .48 \\
\hline
\end{tabular}

\section{Discussion}

Our first aim was to compare OHA adherence between the ProMAS and MARS-5. Overall adherence differed markedly between these questionnaires, with a difference around $40 \%$ in adherence percentages, which is in line with earlier publications $(30,124)$.

Patients are generally considered to be adherent if the adherence percentage to prescribed medication matches or exceeds $80 \%$ (138). This implies a submaximal, but acceptable 
adherence percentage, which leaves room for improvements in patients'adherence. Applying this acceptable principle, the ProMAS adherence percentage of $47.7 \%$ virtually matches the adherence percentage as collected by objective methods, in contrast to the adherence percentage of 89.5 as observed in the MARS-5 (33). As shown in our study and previous application of the ProMAS, when dichotomized, the percentage of patients being adherent is about $50 \%$. These data match data gathered from objective measures, assessing medication adherence of chronic ill patients, or more specific patients applying OHAs such as those assessed by our study. Although we did not use an objective measure ourselves, data on OHA adherence through objective measures is widely available. These data also show that around $50 \%$ of the patients adhered to their OHAs (139-141).

In addition, the ProMAS covers a broader spectrum of adherence behaviours compared to the MARS-5. For instance, additionally to forgetfulness which has been reported earlier as the most common reason for non-adherence (142), the ProMAS also identified incorrect medication timing as a major issue in non-adherence. By contrast, the skewed adherence distribution of the MARS-5 only slightly discriminates between adherent and non-adherent patients. Therefore, current results confirm earlier work in which the ProMAS and MARS-5 are compared and underline the promising character of this novel adherence instrument to assess OHA adherence in type 2 diabetes patients (124).

We observed a moderate to large correlation $(r=67)$ between the ProMAS and MARS-5. This implies that there is a fairly substantial linear coherence between the measures. However, by squaring the correlation, which results in an $R^{2}$ value, a more interpretable inference can be made about their cohesion. This results in an $\mathrm{R}^{2}$ of .45 , which represents a shared variability of $45 \%$ by the ProMAS and MARS-5 score. To put this value into perspective, still $55 \%$ of the variability is left to be accounted for by other variables. This shows that shared variance between the ProMAS and MARS-5 is rather moderate.

Our second study aim was to identify socio-cognitive predictors of OHA adherence using the ProMAS as outcome. In the fourth multivariate model, a low education level, lower severity perceptions, and higher self-efficacy and intention predicted better OHA adherence. A low education level and higher self-efficacy remained significant predictors even after adjusting for baseline behaviour.

While DiMatteo (143) and Kirkman and colleagues (144) associated higher levels of education with medication adherence, a recent study by Chew and colleagues (145) indicated that higher education was associated with poor diabetes medication adherence. Another review by Jin and colleagues (146) reported inconsistent findings. Contradictory findings might be due to variations in study designs and populations, outcome measures applied and analysis methods $(144,145,147)$. However, a potential explanation for the identification of a low education level as predictor of OHA adherence in the current study, is that patients with 
a low education might believe more in their physicians' treatment suggestions (146). As a result, lower educated patients might be more prompted to follow physicians' prescriptions compared to higher educated patients.

Unexpectedly, severity perceptions were significantly inversely related to medication adherence in both bivariate and multivariate analyses; current socio-cognitive models assume that higher levels of perceived threat should result in higher levels of medication adherence $(37,43)$. One potential explanation could be that our analyses were confounded by depression, which was not measured in our study. Depression was in earlier studies associated with higher severity perceptions (130) and with lower self-efficacy and medication adherence (30). According to the Protection Motivation Theory, it is assumed that adverse actions will be preserved if there is a high threat perception but low perceived self-efficacy (148). Clearly further research is needed to explore the inverse relation of severity perceptions and medication adherence.

Self-efficacy has been reported extensively in earlier studies as a factor associated with adherence to diabetes medication $(28,130,142)$. Particularly, lower self-efficacy to adhere to medication tends to occur in out-of-routine situations, e.g. being on vacation, out for dinner, busy or ill $(28,142,149)$. Associating medication taking with everyday activities has been found to aid in $\mathrm{OHA}$ adherence, while a change in daily routine would make adherence more difficult $(142,150)$. Achieving medication adherence in routinely situations, might increase self-efficacy beliefs through mastery expectations. On the contrary, it might be more difficult to achieve medication adherence in out-of-routine situations because no association can be made with everyday activities, given the irregularity of the situation (54). Repeated success, i.e. adherence to medication in situations when routine is disrupted, might increase selfefficacy and in turn medication adherence and should in that regard be facilitated by linking adherence to daily activities $(54,142)$.

\section{Strengths and limitations}

This study is subject to some strengths and limitations. To our knowledge this is the first study that applied a longitudinal study design in assessing socio-cognitive predictors of $\mathrm{OHA}$ adherence and to use the ProMAS to assess adherence. Moreover, this study added a time-frame of three months over which adherence is assessed, which might have decreased recall-bias and provided a more accurate adherence assessment. Limitations include the social desirability issues of self-report adherence measures (129). Despite the considerable advantages of assessing adherence through self-report instruments, a combination of subjective and objective measures is preferred (129) and should, when feasible, be included in future studies assessing adherence. By including both self-report and objective measures, one is able to make mutual performance comparisons. An advantage of applying both measures simultaneously in one study is that results will be obtained from the exact same study population contrary to comparing results across studies and study populations. 
Our approach to predicting OHA adherence assumed that adherence is a rational process, partly explained by socio-cognitions. Although a large part of the variance in adherence behaviour was explained by these socio-cognitions, a substantial contribution to nonadherence behaviour was made by forgetfulness, which is considered unintentional, i.e. nonrational behaviour (151). Hence, future studies should examine a combination of rational processes and processes underlying unintentional non-adherence, to contribute to a potential holistic understanding of $\mathrm{OHA}$ adherence.

\section{Conclusion and recommendations}

Results primarily indicate that $\mathrm{OHA}$ adherence is suboptimal, independent of measurement instrument and cut-off point applied. Our results confirm earlier research on the wide variety of OHA adherence percentages, which largely depends on methodology and cut-off points applied. Consensus on the best method to assess adherence is lacking and no golden standard exists in dichotomizing adherence scores which may hamper interpretability of divergent results. However, when applying sub-maximal cut-off points, which is generally acceptable, the ProMAS yielded results similar to objectively collected data. Moreover, when compared to the MARS-5, the ProMAS yielded less skewed data towards adherence and insights in a broader spectrum of (non)-adherence behaviours. Hence, the ProMAS seems better equipped to deal with flaws present in existing self-report instruments. Adding a relevant time-frame might decrease recall bias and provide more accurate estimates of adherence.

Suboptimal adherence stresses the need for effective adherence improving interventions. Decreasing forgetfulness and medication taking timing issues might increase adherence rates. Interventions should particularly target higher-educated patients and patients with low selfefficacy in out-of-routine situations as these characteristics predict lower adherence levels. Our result regarding severity perceptions requires further inspection to examine potential explanations suggested in this paper. 


\section{My Diabetes}

\section{Profile?!}
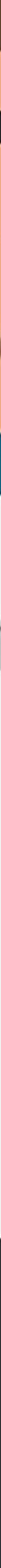


\section{Chapter 4}

A web-based program to improve treatment adherence in patients with type 2 diabetes: Development and study protocol

\section{This chapter has been published as:}

Vluggen S, Hoving C, Schaper NC, de Vries H. A web-based program to improve treatment adherence in patients with type 2 diabetes: 


\section{Abstract}

Background: Many patients with type 2 diabetes mellitus (T2DM) suboptimally adhere to core treatment recommendations, such as healthy diets, sufficient physical activity and pharmacological support. This paper describes the development of the web-based computer-tailored program My Diabetes Profile (MDP), incorporating identified success factors of web-based interventions, and the protocol for testing the effectiveness of this program in a randomised multi-center trial.

Methods: Formative research - including the input of a program committee, qualitative and quantitative studies with patients and health professionals and a literature search - yielded input for the development of the MDP program. MDP provides video and text tailored advice, based on determinants and salient beliefs derived from the I-Change Model, on decreasing unhealthy snack intake, increasing physical activity, and improving adherence to both oral blood glucose lowering drugs and self-administered insulin therapy. Patients with T2DM recruited by practice nurses and diabetes nurses across the Netherlands fill in online questionnaires at baseline and six-months follow-up. Participants are randomized on patient level to the intervention group (access to the MDP program) or control group (receiving care as usual).

Discussion: The formative research using co-creation principles proved essential in the development of the MDP program and involved various disciplines in T2DM management including target group representatives. Cocreation revealed clearly that patients needed short and attractive messages. Consequently, a mix of video and short text messages were chosen for the ultimate program format. Pilot testing was useful to further shape the program to needs of patients and professionals. 


\section{Introduction}

Type 2 diabetes mellitus (T2DM) is a progressive disease characterized by hyperglycemia and the body's inability to maintain a normal glucose metabolism (152). Worldwide over 400 million people live with diabetes, with expectations of almost 650 million people being affected by 2040 (152). Core T2DM treatment recommendations concern lifestyle modifications such as improving dietary patterns and increasing physical activity (PA) as well as pharmacological support such as oral blood glucose lowering drugs and/or (self-administered) insulin therapy $(14,15)$. Unfortunately, patients' adherence to each of these separate recommendations is suboptimal $(23-25,30,102)$. The majority does not consistently meet dietary or PA recommendations (23-25), and most studies on adherence to pharmacological support, report adherence prevalences below 80\% (range 38.5-93.1\%) (30). Suboptimal adherence not only attenuates positive treatment effects (16), but is also associated with disease worsening, an increase in cardiovascular events, quality of life reduction, increased healthcare expenditures and hospitalizations, as well as early mortality $(7,17-22)$. Clearly, new avenues need to be sought to improve treatment adherence in patients with T2DM.

Patients' (non)-adherence to specific treatment recommendations can partly be explained by socio-cognitive determinants, such as a person's knowledge, attitudes, self-efficacy and intention (43). Salient personal beliefs about a certain treatment recommendation, underlying these socio-cognitive determinants, are considered important in predicting treatment adherence $(84,109)$. For instance, patients often lack knowledge and motivation to modify lifestyles, as well as clear goals and coping plans to persist in these changes $(14,63,64)$. Therefore, these determinants need to be addressed in interventions that aim to improve treatment adherence (84).

The Internet offers novel opportunities to improve treatment adherence in chronic diseases such as T2DM (70). E-Health interventions have shown to be (cost)-effective, easy in use, have fewer availability restrictions than regular medical consultations, and can temper pressure on healthcare systems $(73-78,86,153)$. Moreover, these interventions can apply computertailoring technology; a methodology to provide patients with personalised advice based on unique answers given during an online assessment (81). Yet, a recent general review on Internet interventions supporting diabetes management (71) concluded that only one of the nine included studies reported significant improvements in dietary patterns and PA, with small to modest effect sizes of .19 and .32 respectively (79). Furthermore, none of the studies focussing on improving medication adherence yielded significant results. However, existing web-based support programs often include little interactive content, are mainly text-based, make little use of theoretical substantiation, and focus on separate behaviors which play a role in the management of T2DM instead of combining behaviors (71, 80). As success factors of web-based interventions include using a theoretical framework, providing interactive tailored information, applying goal setting principles, using tracking tools, identifying risk behaviors, 
making use of visual support and focussing on various phases of health behavior change (i.e. awareness, motivation and action planning) $(70,71,79,80)$, web-based diabetes treatment adherence interventions effects might be significantly improved by incorporating these factors.

First, this paper describes the development of the new web-based computer-tailored program (My Diabetes Profile), aiming to improve patients'adherence to core T2DM treatment recommendations, by incorporating previously identified intervention success factors. Subsequently, it describes the protocol for the assessment of its effectiveness in a randomized multi-center trial.

\section{Methods and design}

\section{My Diabetes Profile program}

As preparation for the development of the My Diabetes Profile (MDP) program, formative research was conducted. Firstly, both qualitative (28) and quantitative studies were conducted to identify the scope of treatment (non)-adherence and to elicit salient personal beliefs involved in treatment recommendation adherence (109). Findings indicate that patients' adherence to treatment recommendations was suboptimal and therefore subject to improvement. Moreover, many patients incorrectly perceived themselves as adherent to distinct treatment recommendations. With regard to non-adherence to dietary recommendations, patients were most likely to engage in unhealthy snack intake (28). Secondly, knowledge was accumulated from previously developed computer-tailored programs targeting improvements in treatment recommendation adherence $(52,154,155)$. Thirdly, a program committee was formed to foster co-creation, following the principles of Havelock's linkage approach (156). The committee met three times during the 18-months program development phase and included members from various disciplines involved in T2DM management: practice nurses (PNs), diabetes nurses (DNs), a dietician, an internist, a general practitioner, health scientists, an e-Health expert, and patients with T2DM. Based on the input of the formative research and co-creation, the MDP program was developed.

The content of the MDP program is theoretically grounded in the I-Change Model (ICM) (43). The ICM integrates different well-known socio-cognitive theories $(36,37,109)$ and is used often to identify salient beliefs of health behavior (change) and develop interventions accordingly (110). The ICM differentiates between three phases; an awareness phase, a motivation phase and an action planning phase, which are influenced by preceding factors and information factors (see figure 1). The model assumes that behavior change is a result of becoming aware of the necessity of behavior change by activating risk perceptions and increasing knowledge of the behavior and its consequences. Moreover, a person's cognizance level indicates if a person is (in)correctly aware of carrying out the recommended behavior. For instance, a person could 
perceive to be sufficiently physically active, while in fact recommended levels sufficient PA are not met. Contrary, a person could correctly perceive that s/he is not sufficiently physically active, which indicates awareness of the discrepancy between what is recommended and actual behavior. In sum, first one needs to be aware of the necessity of behavior change. Subsequently, if sufficient awareness of behavior change is present, a weighing of the pros and cons of the desired behavior, perceptions of social influences, and the level of one's own belief to successfully carry out the desired behavior in certain difficult situations (self-efficacy), determines the motivation a person has to change a behavior. The strength of the intention to change a behavior a person has, is determined by motivational factors and awareness factors. The ICM assumes that people who express a low intention towards behavior change, can increase their intention to change by increasing their motivation and awareness of a specific health behavior. Contrary, people who express a high intention towards behavior change have a higher likelihood of successful translation of this intention into practice, by making and enacting action and coping plans. In this phase, again self-efficacy plays a major role in carrying out action plans. It is well known that expressing a high intention towards behavior change, does not necessarily guarantee successful behavior change (56). Hence, the action phase facilitates the translation of intention into actual action.

Figure 1 The l-Change Model

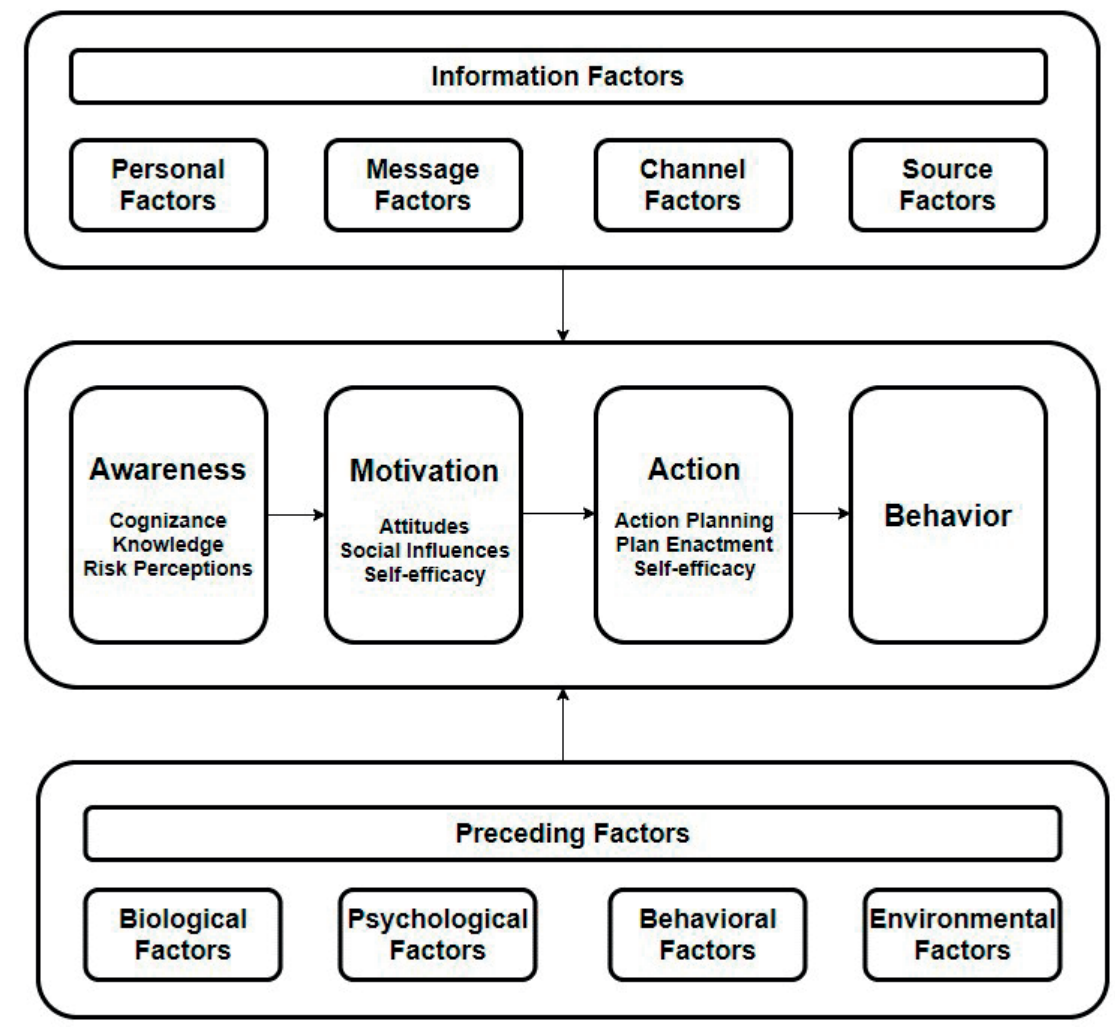




\section{Program content}

The MDP program starts with a baseline assessment consisting of demographic questions, questions on comorbidity, patient's perceived adherence (cognizance) to separate treatment recommendations, and an objective treatment recommendation adherence assessment regarding $\mathrm{PA}$, unhealthy snack intake, medication adherence (adherence to oral blood glucose lowering drugs and/or self-administered insulin therapy), and smoking. The objective treatment recommendation adherence assessment will serve as outcome measure and is described in more detail in section 2.3.2.

The program lasts a total of six months, and consists of two practically identical blocks of three months. In each block of three months, participants can select a single treatment recommendation to improve. For instance, it might be that a participant selects unhealthy snack intake to improve in the first three months, and physical activity in the second three months. However, participants are able to select the same behavior again in the second three months if they believe this particular recommendation needs further improvement relative to others. In turn, every three-month block consists of three sessions; 1) health risk appraisal, 2) awareness and motivation, and 3) goal setting and action planning, which guide the behavior change process. All sessions last on average 20-30 minutes and can be continued any time where someone left off. 
Figure 2 Schematic overview of program course

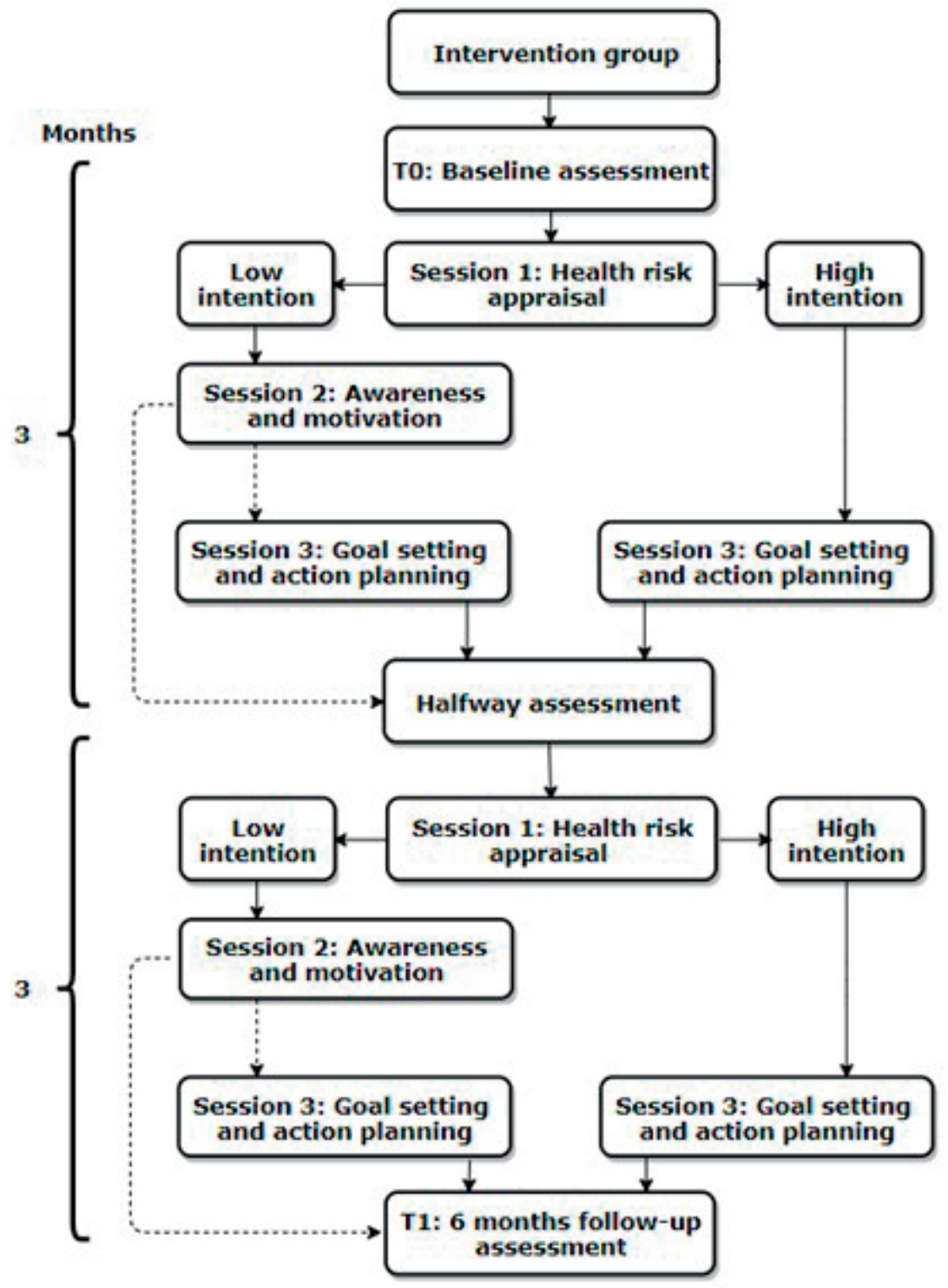

\section{Session 1: health risk appraisal}

In the first session, participants receive a tailored health risk appraisal based on the answers given to the baseline assessment. The health risk appraisal provides information on whether their perceived adherence to treatment recommendations matches with objective guideline targets. For those behaviors that the participant is not yet adherent to, their intention to change that behavior is assessed. As the last parts of session one, participants are prompted to self-select a single behavior that can be improved and which will be their focus for the coming three months while working with the program. The approach of selecting a single improvable behavior at a time was chosen because on the one hand patients are more 
successful in achieving behavior change in one treatment element, when compared to two (116). On the other hand, improving behaviors sequentially rather than simultaneously, might work better if patients are permitted to self-select a behavior to work on first and which aligns best with their willingness to change (157). Although smoking status is assessed and followed-up with tailored advice in the health risk appraisal, it is not considered a core T2DM treatment recommendation $(14,15)$. Hence, participants are not able to select this behavior within the MDP program. Instead, all smokers receive a brief advice to quit and are directed to their general practitioner for further information and support. In case participants meet all recommendations they are recommended to select PA, as current Dutch guidelines state that any physical activity beyond the weekly recommended 150 minutes is desirable (158). A participant who has selected a behavior for which s/he has a low intention to change (i.e. not motivated to change, or motivated to change but not within the next three months) is directed to session two (i.e. awareness and motivation). This session particularly aims to increase this motivation or raise awareness for the need to make improvements, with the ultimate goal to achieve a high intention to change. Therefore, session two is designed for participants to keep on working with the program procedure, even while they express a low intention to change an improvable behavior. If a behavior is selected for which the participant has a high intention to change (i.e. motivated to change within three months), the participant is directed to session three (i.e. Goal Setting and Action Planning). This strategy was chosen to optimally tailor the intervention to the behavior change state of the participant as assumed by the l-Change Model (43).

\section{Session 2: awareness and motivation}

The second session consists of two sub-sessions and is specifically designed for participants who chose to work on a behavior for which they have a low intention to change.

The goal of sub-session 2a is to increase a participant's motivation to change behavior by providing tailored advice on the motivational determinants 'attitudes' (i.e. pros and cons) and 'perceived social influences' (i.e. social support, social modeling and social norm). First, salient beliefs with regard to motivational determinants are assessed on a 5-point Likert scale (totally disagree - totally agree), after which tailored feedback is provided. Session 2a ends with again an assessment of the intention to change the behavior. If a participant now has a high intention to change the behavior, the participant will be directed to session 3. If a participant still holds a low intention to change, s/he continues to session $2 \mathrm{~b}$.

The goal of sub-session $2 \mathrm{~b}$ is to increase a participant's awareness to change behavior by providing tailored advice on the awareness determinants 'knowledge' and 'risk perception'. Salient beliefs with regard to risk perception are assessed on a 5-point Likert scale; knowledge items are answered on a dichotomous scale (true, false). Session $2 \mathrm{~b}$ ends with a final assessment of the intention to change the behavior. If a participant now has a high intention, the participant is directed to session 3. If a participant again has a low intention to change 
the behavior, the program advises to wait for the second block of three months. Participants are then able to select a different behavior to work on or to use the time in between to contemplate changing the initial selected behavior.

The approach to start with increasing motivation and if necessary raising awareness was chosen to keep the program as proximate to behavior as possible according to the ICM. At the same time, the aim was to offer only the most relevant feedback and content to patients, in order to keep the total time to use the program low and feedback tailored to the individual user's needs.

\section{Session 3: goal setting and action planning}

In the third session, participants are primarily prompted to set specific goals for their selected behavior. For instance, participants who wish to increase their level of PA can select how many more minutes/week they want to be physically active. The program facilitates goal setting by providing several pre-formulated options varying in difficulty, but also provides an option to construct their own goal. Participants are encouraged to set small, realistic and achievable goals (116). Next, participants select pre-formulated action plans or construct their own. For instance, if a person wants to become more physically active, the program facilitates goal achievement likelihood by formulating specific plans on how to become more physically active, e.g. taking the stairs more often or cycling to work. Subsequently, self-efficacy is assessed, i.e. participants are asked to identify pre-formulated or individual situations in which they might find it difficult to carry out the intended goal, e.g. being physically active when it rains. Self-efficacy is assessed on a 5-point Likert scale (very difficult - very easy). This is followed by tailored advice on why particularly these situations are considered difficult and how to deal with them. Last, participants are prompted to formulate specific pre-formulated or individual coping plans for their difficult situations. Session 3 ends by providing patients with an overview of the advice they received and the goals and plans they made. Participants can now revisit their advice at any time, as well as the general program modules in the main menu.

\section{Forms of computer-tailoring}

The MDP program offers two forms of tailored advice, video and text tailoring, throughout all sessions. First, participants receive segment-tailored information messages via video, to generate attention and to help process information (80). Tailoring based on segmentation entails that participants who score high, medium or low on a specific determinant receive different video content. For instance, a participant that scores high on pros of PA (i.e. seeing many advantages to being physically active) receives a video that reinforces the advantages a participant sees and provides additional advantages. Contrary, a video for participants who score low on pros of PA (i.e. perceiving few advantages to being physically active) includes arguments to persuade participants of the most salient advantages of PA. All videos are animated video's created with Go Animate software (159). Animated videos were preferred by the program committee over real life videos because they were perceived as more exciting 
and potentially less confronting to patients with T2DM. After a video, participants receive a brief text advice, which generally consists of five to ten text lines with more in-depth tailored information. For instance, the health risk appraisal text on PA provides accurate numbers of minutes a participant is physically active. Keywords in the text messages are either underlined or highlighted by color or font to generate additional attention (160).

\section{Ipsative advice}

The health risk appraisal (session 1) at three-month follow-up (i.e. the start of the second three-month block) uses both the answers given to the baseline assessment and the answers given to the halfway assessment to provide ipsative advice (161). Ipsative advice can be offered in interventions with multiple feedback sessions in time and can provide insights in the direction and extend of changes over time in relevant behaviors (161). The advice is displayed in tailored text messages, which provide the participant information on whether s/ he significantly improved, deteriorated or did not change a certain treatment element.

\section{General modules}

In addition to tailored behavioral modules, the MDP program also includes three general modules; My Care, My Values and My Profile. These general modules were incorporated to make the program a more complete self-management tool, to facilitate consultation preparation, and to stimulate program revisits. My Care facilitates participants in preparing their consultations and stimulates participants to proactively formulate questions, provide a brief health update, and to learn how to be an active collaborator in consultations. My Values gives participants the opportunity to enter and visually keep track of their blood glucose levels, blood pressure, weight, and body mass index. My Profile provides a clear overview of the participant's most recent entered values, their medication, and their next appointment with their healthcare professional. Figure 3 displays the main menu of the MDP program. 
Figure 3 Main menu of the My Diabetes Profile program

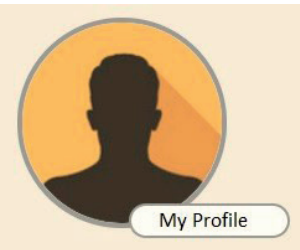

Click on the button for more information:
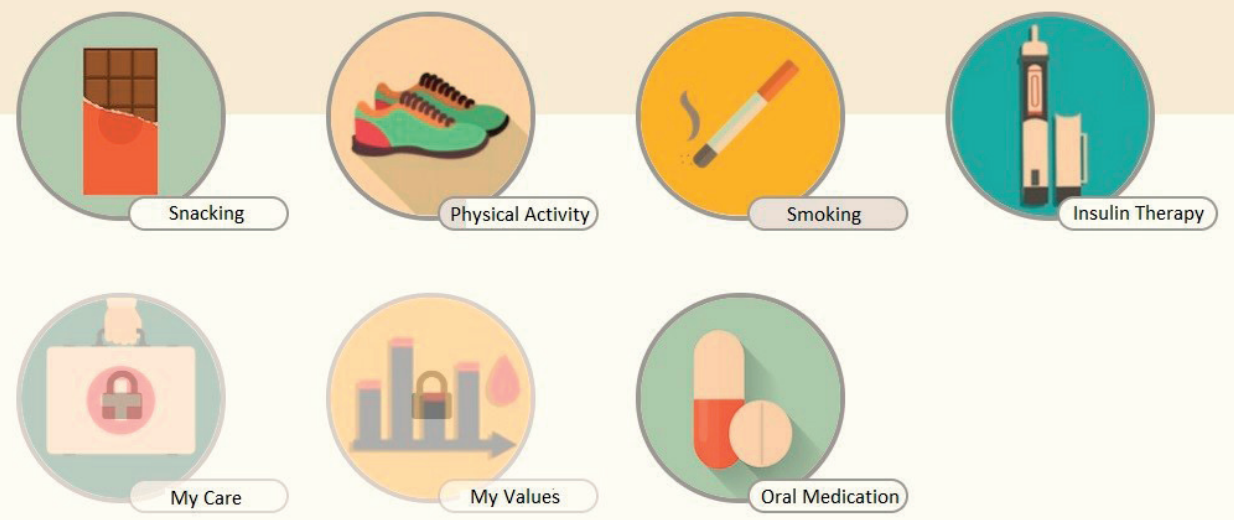

\section{Health report}

In order to facilitate discussion of respondents' health goals during regular medical consultations, the healthcare professional will have access to the patients' individual health report summary. The health report visually displays the behavior the respondent has selected to improve, the goals s/he has set, and the information from the My Care and My Values modules.

\section{Usability testing}

To identify potential program bugs, to assess participants' satisfaction with the program, and to make pre-trial improvements, usability tests were conducted with healthcare professionals $(n=5)$ and T2DM patients ( $n=17)$. Recruitment occurred pragmatically in one outpatient clinic, where healthcare professionals and patients were deliberately recruited, taking into account profession, age, gender, education level, medication use, to strive for heterogeneity. Healthcare professionals were provided with login-data and program instructions, and were asked to visit the program website. Patients were provided with login-data and program instructions and were asked to use the program. While reading the instructions and visiting the program, healthcare professionals and patients were instructed to express their thoughts and opinions using the thinking aloud method (162). Usability tests were conducted at the research department in the presence of a researcher and were audio recorded. Expressed thoughts and opinions were used as input to improve the program. Healthcare professionals 
and patients reported that they were very satisfied with the program and its usability. Only minor changes had to be made, which included instructions on how to navigate in the program, on how to answer questions on PA adherence, and on how to thoughtfully read questions and information.

\section{Prompts to promote program use}

The My Diabetes Profile program includes periodic email prompts to persuade participants to (re-)log in and to enhance usage. Periodic prompts are considered promising in enhancing effectiveness (163) and short timing prompts (two weeks after their first visit) seem to be most effective in encouraging computer-tailored program revisits (164). The prompts used in our program are tailored to the participants' gender and last name. Participants receive a maximum of two email reminders, within two weeks after their most recent visit, to prompt completion of a yet uncompleted session, or when new sessions or assessments become available.

\section{Study design effectiveness trial}

A multi-center randomized trial with a waiting list control group design will be used to assess the effectiveness of the developed MDP program in patients with T2DM. After recruitment, registration and informed consent procedure, all participants will be randomly assigned to either an intervention group or control group. Group allocation takes place on patient level by means of computer randomization which allocates $50 \%$ of the participants to either group. Respondents of both groups are then prompted to fill in an identical baseline assessment (TO). Intervention group participants then gain access to the MDP program for six months. Control group participants are made aware that they will not receive access to the program at this stage, but are eligible for delayed access, after completion of the effectiveness trial (waiting list control group).

Six months after baseline completion, all participants are prompted to fill in the follow-up assessment (T1). Afterwards, control group participants will receive instructions on how to request program access. The study is approved by the Medical Ethics Committee of the Maastricht University Medical Center (16-4-171). The study is registered in the Dutch Trial Register (NTR6840).

\section{Recruitment of T2DM participants}

In the Netherlands, T2DM patients are predominantly treated by practice nurses (PNs) and diabetes nurses (DNs) in the general practice and hospital outpatient setting respectively, who are in turn supervised by physicians (14). Most T2DM patients visit their PN or DN quarterly, and in case of no direct risk factors of health complaints every semester (14), hence these healthcare professionals are in an ideal position to recruit patients for our trial. Trial inclusion criteria are: 1) T2DM diagnosis for at least one year, 2) 40-70 years old, 3) using at least one form of diabetes pharmacological support, and 4) having no walking disability. Exclusion criteria 
are: 1) not speaking or understanding the Dutch language, 2) having no access to the Internet, and 3) insulin pump therapy. People on insulin pump therapy will be excluded because of the distinct adherence nature of insulin pump therapy compared to self-administered insulin therapy. Given this recruitment strategy, patients are nested in the general practice and hospital outpatient settings.

PNs and DNs across the Netherlands will be approached through email (with an invitation and brochure that explained study details), telephone calls, letters to their work address and social media platforms. PNs and DNs can register for the study by contacting the research team directly, or by registering via the project website. PNs and DNs are asked to recruit T2DM patients during regularly occurring medical consultations, through telephone calls or through email for a period of six-months. Prior to the start of the effectiveness trial, participating healthcare professionals are provided with detailed information about the study and the MDP program. A recruitment checklist can be used during consultations to facilitate recruitment and graphically depicts the study's inclusion criteria. The checklist also includes the healthcare professional's unique username and password, and instructions on how to register a participant. Following registration by their PN or DN, patients immediately receive an email from the research team including login instructions and an invitation to fill in the online baseline assessment. After logging into the system, patients are provided with extended study information and an online informed consent form. After signing for informed consent, participants are directed to the baseline assessment.

\section{Power calculation and data collection \\ Primary outcome measure and power calculation}

To assess the effectiveness of this multiple behavior intervention program as a whole, changes in adherence to PA, unhealthy snack intake, oral blood glucose lowering drugs and self-administered insulin therapy will be standardized and summed into a composite lifestyle index score as primary endpoint. As proposed by Prochaska and colleagues (165), an option to create standardized change scores is to subtract baseline scores for each behavior separately from follow-up scores and subsequently divide this by the standard deviation of the difference of the specific behavior. This way, effect sizes can be calculated for all behavior separately. Subsequently, to create the index score, separate effect sizes for all behaviors were summed. The effect size for unhealthy snack intake was reverse coded since the intervention aimed to decrease this behavior. To assess significant change between the intervention and control condition, a two-tailed T-test was applied with an a of $5 \%$.

Our power calculation is based on the primary outcome measure and aims for a near medium MDP program effect size of 0.4. Assuming an intraclass correlation coefficient of 0.02 , an a of $5 \%$, and power of $0.80,116$ patients per group are required at the end of the trial (232 in total). Considering an attrition percentage of $50 \%$, our aim is to include a minimum of 464 patients 
at baseline. We expect that each PN/DN can recruit eight patients, hence we aim to recruit a minimum of 58 healthcare professionals.

\section{Data collection}

The data obtained from the first entry into the program, as described earlier in section 2.1.1 (Program Content), will be used as baseline assessment. These measurements will be repeated at a six-months follow-up assessment except for the comorbidity questions. Socio-cognitive determinants are assessed with four to six items on a five point Likert scale (totally disagree totally agree). We use the following instruments to measure our primary outcomes.

\section{Physical activity}

The level of PA is assessed using the validated Short Questionnaire to Assess HealthEnhancing Physical Activity (SQUASH) (166). The SQUASH assesses physical activities in the area of commuting, leisure time and sports, household, and work and/or school. Activities can be reported in average hours and minutes per day, and the frequency of weekdays these activities are carried out. A total number of weekly minutes of physical activity will be calculated based on the SQUASH.

\section{Unhealthy snack intake}

A food frequency questionnaire (FFQ) developed for this trial is used in which participants are asked to recall their last week's unhealthy snack intake. The FFQ lists unhealthy snacks identified by earlier research supplemented with unhealthy snacks which are commonly consumed in the Netherlands $(167,168)$. All listed unhealthy snacks account for a specific amount of caloric intake which will be summed to create a total number of weekly calories consumed from unhealthy snacks.

\section{Oral blood glucose lowering drugs adherence}

Oral drug adherence will be measured with the ProMAS questionnaire (124), a self-report instrument which has been recently developed in response to flaws in existing and frequently used self-report adherence measures such as the MARS-5 and Morisky Medication Adherence Scale $(124,169)$. It consists of eighteen items that assess a variability of adherence behaviors, e.g. forgetting, stopping, changing dosages, or taking medication too late (range 0-18). A higher score indicates a better adherence. In close collaboration with the developers, we translated the original English ProMAS questionnaire to Dutch which fits the use in this intervention study. To be able to compare changes over time, we further added a time period to every single items to assess adherence of the past three months. Based on the eighteen items, a sum score will be calculated as outcome for adherence to oral blood glucose lowering drugs.

\section{Insulin therapy adherence}

Adherence to insulin therapy is measured with a self-administered questionnaire, adapted from the ProMAS questionnaire (124). Non-relevant items were removed, resulting in a 
measure with nine times. Items were considered not relevant if they did not distinguish between adherence and non-adherence for insulin therapy (e.g. the item 'I sometimes take fewer medicines than prescribed by my doctor' was removed as patients using insulin should adjust their dosage in case of hypoglycemia or illness). In line with the assessment of oral blood glucose lowering drugs adherence, a time period was added to every single item to assess adherence of the past three months. Based on the nine items, a sum score will be calculated as outcome for adherence to insulin therapy, ranging from 0-9 where higher scores indicate better adherence.

\section{Data analysis}

Complete case analyses will be conducted for participants who completed both the baseline and follow-up assessment. Sensitivity analyses will be carried out for those who did not complete the follow-up assessment, i.e. for participants of which no primary outcome is available at follow-up. For incomplete assessments, multiple imputation on the outcome measure will be applied using both an optimistic and pessimistic scenario.

Descriptive statistical methods and frequencies will be used to describe sample characteristics. A logistics regression analysis will be conducted to identify potential selective attrition of participants between baseline and follow-up with regard to demographics. The effect analyses will be conducted for the primary outcome measure which will consist of a combined index of individual behavior z-scores. Given the hierarchical structure of the data, multilevel linear regression analyses will be conducted. Besides the repeated measures structure, patients are nested within healthcare providers, and two types of healthcare providers will recruit the participants, i.e. practice nurses and diabetes nurses. Therefore, these levels will be included in the analyses as random factors. Both adjusted and un-adjusted analyses will be provided and analyses will be adjusted for potential confounders (i.e. baseline adherence, predictors of attrition, and demographics). All statistical analyses will be executed using SPSS version 24.0.

\section{Discussion}

This paper presented the design, course and content, and proposed effect evaluation protocol for a newly developed web-based computer-tailored program named My Diabetes Profile. The program aims to improve patients' adherence to core T2DM treatment recommendations and incorporates previously identified success factors to successfully influence patients'adherence rates. Because patients' adherence to treatment recommendations is frequently suboptimal, interventions are needed to support treatment adherence and to support the persistence of behavior over time. The Internet offers promising opportunities to intervene given a variety of advantages including the potential to reach out to the rapidly growing population of patients with T2DM, the potential (cost)-effectiveness of Internet based interventions, the application of computer-tailoring technology and the tempering of pressure on healthcare 
systems. The MDP program combines various success factors such as using a theoretical framework, providing interactive tailored information, applying goal setting principles, using tracking tools, identifying risk behaviors, making use of visual support and brief text advice, and focussing on various phases of health behavior change. Hence, we hypothesize that the MDP program can effectively improve treatment recommendation adherence in patients with T2DM, which will be tested in a randomised trial.

\section{Potential strengths of the study}

Several potential strengths of the MDP program and the study are worth mentioning. First, the program's design, course and content are based on extensive formative research including co-creation by a variety of key persons involved in diabetes care. Secondly, the MDP program incorporates previously identified success factors and uses salient personal beliefs in changing behavior, i.e. improving treatment recommendation adherence. Third, in order not to further burden health care professionals, the program is run completely independent of healthcare professionals in the trial. Fourth, the trial will be conducted nationwide, in both primary and secondary care, which increases generalization of the results obtained. Fifth, the program was comprehensively pilot tested among the target group which yielded useful input for preimplementation improvements. Last, randomization will occur on patient level which means that healthcare professionals include patients which will randomly be assigned to either the intervention or control group. Contrary to randomization on healthcare professional level, this procedure attempts to minimize the potential influence of healthcare professionals.

\section{Potential limitations of the study}

The present study (design) and program is also subject to some limitations. A first potential limitation concerns high attrition rates of participants in online interventions $(80,170)$. Various factors might positively contribute to attrition rates, e.g. a high workload and limited usability of the intervention, and the ease of ending program and trial participation (170). In the program, workload was limited by creating fairly short sessions (20-30 minutes) and allowing participants to access and continue the program at any time where they left off. Moreover, we tried to minimize usability issues by comprehensively testing the intervention prior to the start of the effect evaluation in which participants indicated their satisfaction and by using prompts to pursue program revisits and continued use once a patient is registered. Although these measures are likely to limit the drop-out rate, we still expect considerable drop-put and adjusted our power calculation accordingly (i.e. 50\% drop-out rate at the time of last followup). A second potential limitation is the relatively short follow-up assessment of six months following intervention start. This way, it is not possible to assess if potential program effects sustain over time after program completion. Future studies should, if possible, apply study designs with extended follow-up periods to assess whether potential effects sustain over a longer period of time after intervention completion. Last, as our recruitment period will last for six months, and our intervention duration is six months, this might lead to seasonal variation in our outcome measures. Indeed, Ma and colleagues (171) showed that caloric intake was 
higher in autumn compared to spring and that PA levels were lowest during winter whereas they were highest in springtime. However, seasonal differences in this particular study were considered generally small.

\section{Lessons learned}

As advocated in Havelock's linkage approach (156), the involvement of relevant stakeholders was an essential element in the development of our computer-tailored program and the subsequent design of the trial. Based on our positive experiences we suggest that the program committee should be a representation of all relevant disciplines involved. Selecting one or two individuals, however, does not guarantee that all the relevant viewpoints that are current in a specific discipline are expressed. Therefore, we suggest that in future projects the representatives should, if possible, consult a larger group of colleagues, to seek input on the topics discussed and decisions made by the program committee. This requires not only the formation of a program committee, but also the formation of a number of stakeholder-specific panels. Moreover, our program committee met three times during the formative research and program development phase which lasted for eighteen months. However, it is unclear what the optimal frequency of such gatherings should be and which disciplines should always be involved. We suggest that future projects should properly investigate which disciplines to include and internally assess the necessity and frequency of gatherings.

\section{Conclusion}

This paper offers detailed insight into the design and protocol for the effectiveness trial of the newly developed My Diabetes Profile program. The effectiveness trial's data collection is expected to be available from the end of 2018. If the MDP program is effective in improving treatment adherence in patients with T2DM, adaptation of the intervention to other chronic diseases might have potential value for other patient groups. 


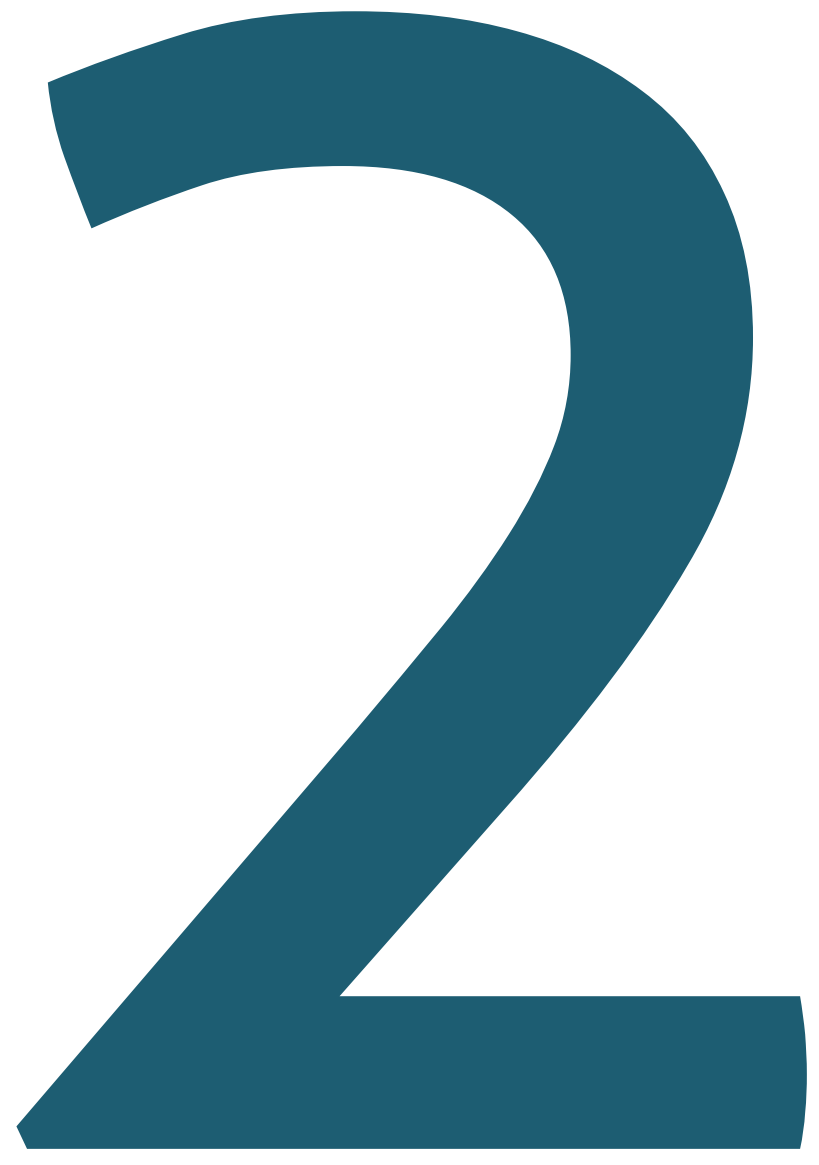




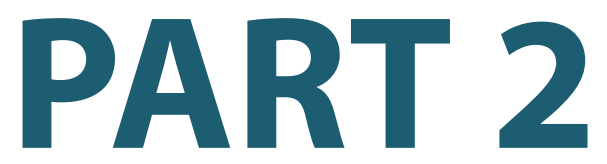

Recruitment and effectiveness

evaluation 


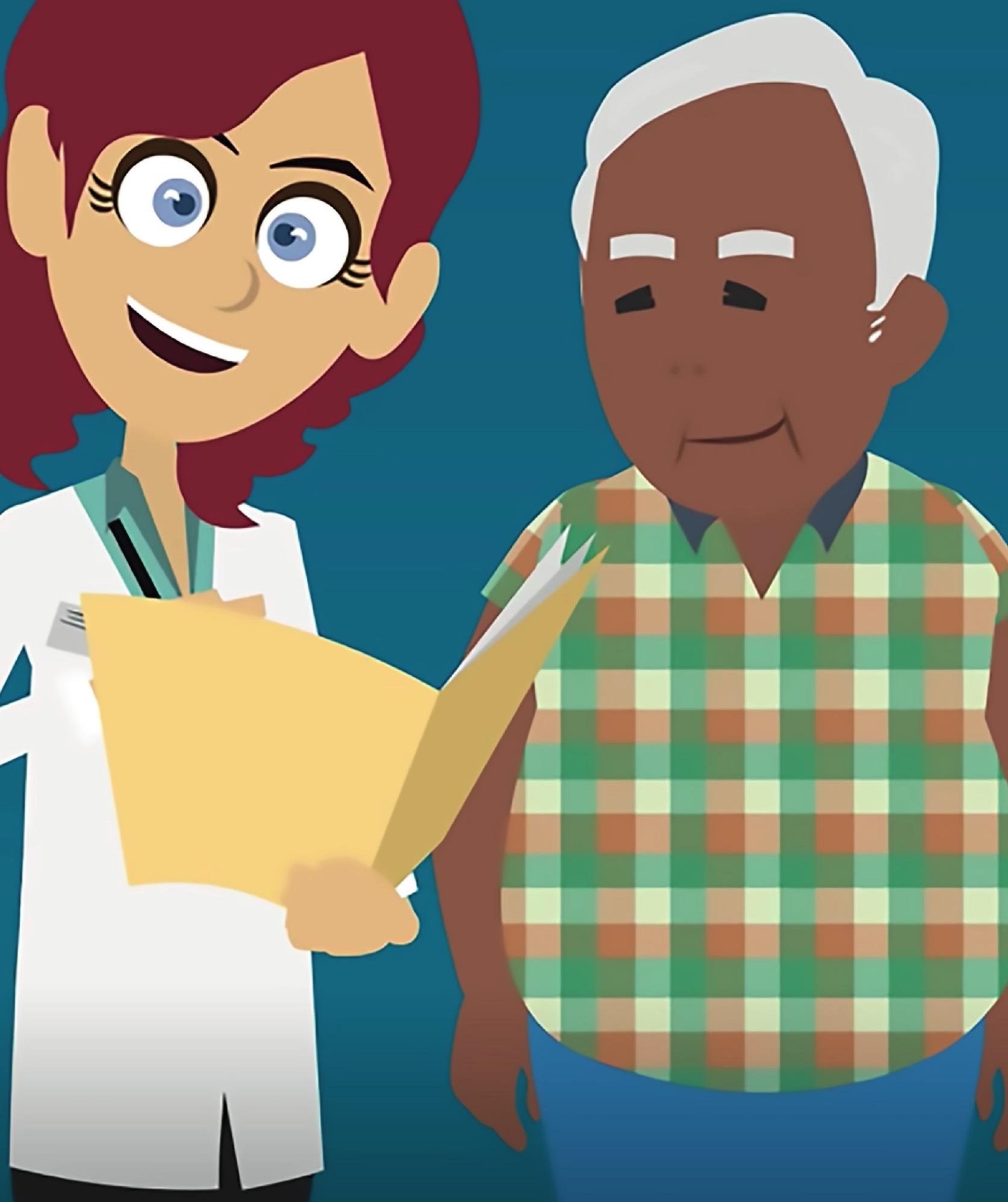




\section{Chapter 5}

\section{Exploring factors influencing recruitment success of nurses recruiting diabetes patients for a randomized controlled trial}

This chapter has been submitted as:

Vluggen S, Hoving C, Vonken L, Schaper NC, de Vries H. Exploring factors influencing recruitment success of nurses recruiting diabetes patients for a randomized controlled trial 


\section{Abstract}

Background: Effective patient recruitment by health professionals is challenging but pivotal to the success of clinical trials. Many trials fail to include the required number of participants which impacts the study power, generalizability of results, and timely dissemination of positive outcomes. Current is inconclusive regarding factors influencing recruitment success, and most research does not rely on perceptions of recruiting health professionals themselves. Therefore, thorough evaluations of recruitment strategies applied in trials are needed in order to define intervention targets to optimize future patient recruitment in trials. We observed divergent recruitment success among nurses who recruited diabetes patients to our trial testing the effectiveness of an eHealth program. Therefore, we aimed to describe nurses'level of recruitment success and its shifts over time, and to qualitatively explore factors influencing their recruitment success.

Method: Nurses'recruitment success and its temporal shifts were derived from trial data (NTR6840). Based on their recruitment success, nurses were categorized as non-, low-, medium-, and high-recruiters. Subsequently, a subset of nurses per group participated in a semi-structured telephone interview. Interviews were analyzed using Nvivo software applying an inductive coding approach.

Results: Ninety-six nurses participated in our trial and recruited on average seven patients (range 0-32). Fifteen nurses did not recruit any patient. Most patients were recruited close to recruitment onset. Nurses who did not recruit patient close to recruitment onset, ended up recruiting no patients. Data shows a relative high early recruitment success that progressively declined over time; high-recruiters were generally successful recruiters throughout the entire recruitment period. Recruitment barriers and facilitators comprised of organizational, study, patient and especially recruiter characteristics. Contrary to non- and low-recruiters, medium- and highrecruiters reported more in-depth knowledge about the study and trial requirements, expressed more personal participation benefits and fewer barriers, and incorporated more recruitment activities, reminders, and barrier-focused coping strategies.

Conclusion and implications: Intervention targets to optimize patient recruitment to clinical trials include the continuation of including recruiters after initial recruitment onset, and encouraging early recruitment success. A personalized approach may aid recruiters to become and remain successful. Primarily it is important to provide recruiters with sufficient information on trial requirements and to address salient benefits for participation of themselves and their patients in the trial. Last, teaching recruiters skills on how to overcome barriers may further enhance motivation and recruitment capacities. 


\section{Background and aims}

Recruiting sufficient patients for randomized controlled trails (RCTs) is challenging, but pivotal to the success of research (91). Many trials do not achieve their required sample size, which can jeopardize generalizability of results and the study's statistical power, reduce study efficiency due to extended recruitment time, and lead to premature trial discontinuation (90, 92-94). Moreover, the dissemination of potential effective treatments to the general population may be delayed (95). Current research highlights the paucity of conclusive evidence regarding factors influencing recruitment success (96). Therefore, researchers have been called to thoroughly evaluate their applied recruitment strategies in order to optimize future patient recruitment and timely translate positive results into practice (91).

Often patient recruitment manifests through efforts of health professionals, as they are relevant contact points for patients $(89,90)$. Whether professionals enroll a sufficient number of patients depends on multiple factors including organizational, study, patient, and recruiter factors $(96,172)$. Organizational factors entail the number of patients seen by professionals, time constraints, and the institutional culture regarding research $(89,92,172,173)$. Study factors include the strictness of trial eligibility criteria, the recruitment protocol, the informed consent procedure, and support from the research team $(89,172)$. Patient factors comprise of patients' interest to participate, while recruiter factors include the professionals' enthusiasm to contribute to successful research, required knowledge and skills, and their intention to execute recruitment tasks $(89,90,173)$. For instance, it is well-known that not all professionals who intend to recruit patients, will actually succeed (89). However, most research on factors of patient recruitment by professionals relies on evidence gathered through narrative reviews or conclusions drawn by researchers, rather than on experiences of relevant professionals themselves $(172,174)$. Perspectives of recruiting professionals might not only provide insights in the importance of previously identified as well as additional factors influencing recruitment success, but might also identify how changeable these factors are and how they can best be addressed in order to enhance success.

Recently we conducted a six-month RCT in primary and secondary care to examine the effectiveness of a novel eHealth program, in comparison to a control group. The program aimed to improve patient adherence to type 2 diabetes mellitus (T2DM) treatment recommendations, i.e. healthy lifestyle changes and pharmaceutical strategies $(14,15)$. Patients were recruited by their practice nurse or diabetes nurse which are their primary contact points of care in the Netherlands (14). After recruitment completion, we observed considerable variations in recruitment success among nurses. This triggered us to thoroughly evaluate recruitment success and to explore factors possibly influencing recruitment success.

This study aimed to 1) describe nurses'recruitment success in our trial, i.e. the number of patients recruited per nurse, and potential temporal shifts in recruitment success, and 2) qualitatively 
explore factors influencing recruitment success as perceived by nurses themselves, in order to define potential targets for improving patient recruitment in comparable trials.

\section{Methods}

\section{Study design}

Trial data was used to describe nurses' recruitment success and its potential temporal shifts. Subsequently, an interview study was conducted among a subset of nurses who engaged in patient recruitment. The study was approved by the Medical Ethics Committee of the MUMC+ (18.4.129).

\section{Patient recruitment strategy}

Nurses working in primary or secondary care across the Netherlands were approached through email, telephone calls, letters, and social-media platforms, to recruit at least eight patients within a period of six months. Nurses were able to sign-up until two months before the end of the patient recruitment period (November 2017-May 2018).

Patient eligibility criteria included: T2DM diagnosis $\geq 1$ year, 40-70 years old, using at least one form of diabetes pharmacotherapy, and having no walking disability. Exclusion criteria included: not speaking or understanding the Dutch language, having no access to the Internet, and applying insulin pump therapy.

The patient recruitment procedure was pilot tested to last approximately three minutes and subsequently included: program log-on, checking the patient's eligibility, informing, persuading and asking the patient to participate, and (if positive) patient registration. To support this process, nurses received instruction materials prior to recruitment onset. Once registered, patients received log-on data, additional information, and time to consider definitive participation. If positive, consecutively the informed consent procedure, randomization, and baseline assessment occurred. The program development and study protocol are described elsewhere (175) and effectiveness results will be published separately.

The power calculation revealed that 116 patients with a completed six-months followup assessment were required per trial arm to detect a clinically meaningful difference in overall patient adherence (175). As we expected patient attrition of 50\% between baseline and follow-up assessment completion, we aimed to include 464 patients with a completed baseline assessment. As not all patients were expected to participate after being recruited by their nurse, we aimed to initially recruit approximately 700 patients, i.e. 150\% of the required 464. For the current analyses, a recruited patient was defined as a patient recruited to the program by their nurse, independent of ultimately consenting or completing the baseline assessment. 


\section{Procedure interviews}

Nurses were divided into four recruitment groups, i.e. non-, low-, medium-, and highrecruiters, based on their markedly differing recruitment success. Four nurses from each group were approached by email to participate in a telephone interview. In case of refusal or non-response, randomly another nurse from the same group was approached. After conducting approximately three-quarters of the initially planned interviews, data saturation was considered reached. Subsequently, no additional interviews were conducted on top of the sixteen planned interviews. Interviews were scheduled in May-June 2018, lasted 15-30 minutes each, were conducted by SV and LV, and were audio recorded.

\section{Measurement}

The interviews were semi-structured using a one-page topic guide that was based on the grounded theory approach, reflected by a flexible and open interview structure (176). Prearranged topics included questions on demographic characteristics, and on organizational, study, recruiter, and patient factors. Organizational factors included topics such as work pressure and the organizations attitude towards eHealth. Study factors entailed knowledge concerning trial and program requirements. Recruiter factors included motives for signingup and the implementation, course, and facilitation of patient recruitment. Patient factors comprised of their motives to accept or decline participation from the recruiter perspective.

\section{Analyses}

Recruitment success and interviewee sample characteristics were described using frequencies in SPSS software. All interviews were transcribed verbatim, anonymised and analysed using Nvivo10 software (112). Content analysis was performed by SV and LV and was guided by the coding process for inductive analyses (177). An inductive coding approach is common in qualitative data analyses, when having applied a grounded theory perspective (176). First, transcripts were closely read through and discussed among both researchers. Second, transcript segments were highlighted if considered to contain meaningful information related to the study objectives. Next, segments were labelled into over twenty categories. Subsequently, in order to limit redundancy and overlap, the number of categories was reduced to approximately ten. These categories were then entered in Nvivo as 'nodes' to which relevant text segments were assigned. Last, the number of categories was decreased to four main topics: 1) organizational, 2) study, 3) recruiter, and 4) patient factors. SV coded all the interviews, while LV coded two randomly selected interviews from each recruitment group. Subsequently, the intercoder reliability and Cohen's Kappa were assessed using the coding comparison query. This resulted in a percent agreement of $99 \%$, reflecting that interview passages were assigned to the same nodes by both researchers in $99 \%$ of the cases. The Cohen's Kappa showed to be 0.83 , reflecting a good strength of intercoder agreement $(112,113)$. 


\section{Results}

\section{Recruitment success}

Ninety-six nurses from across the Netherlands signed-up for participation. Seventy-four nurses were involved immediately from the start, the other twenty-two enrolled later and had an average recruitment period of 2.8 months. The total recruitment period varied between nurses from two-six months. Consequently, we standardized nurses' recruitment success to an average monthly number of recruited patients.

In total, the 96 nurses recruited 669 patients. Of these, 538 (80\%) were recruited by the 74 nurses who were involved from the start, and 131 (20\%) by those 22 who were later involved. Nurses' absolute recruitment success ranged between 0-32 patients and on average, nurses recruited seven patients ( $S D=7.2$ ). About one-third (36\%) reached the target of at least eight patients and fourteen nurses (15\%) did not recruit any patient. When standardized, nurses recruited an average number of 1.2 (range 0-6.5) patients per month as presented in figure 1.

The division of nurses in groups was both pragmatic and statistical in nature. The first group consisted of nurses who did not recruit any patient (non-recruiters, $n=14$ ). The remaining nurses were divided into three fairly equal sized groups recruiting an average monthly number of 0.2-0.7 patients (low-recruiters, $n=27$ ), 0.7-1.7 patients (medium-recruiters, $n=28$ ), and 1.76.5 patients (high-recruiters, $n=27$ ). Medium-recruiters recruited approximately half a standard deviation around the standardized average number of 1.2 patients per month. 


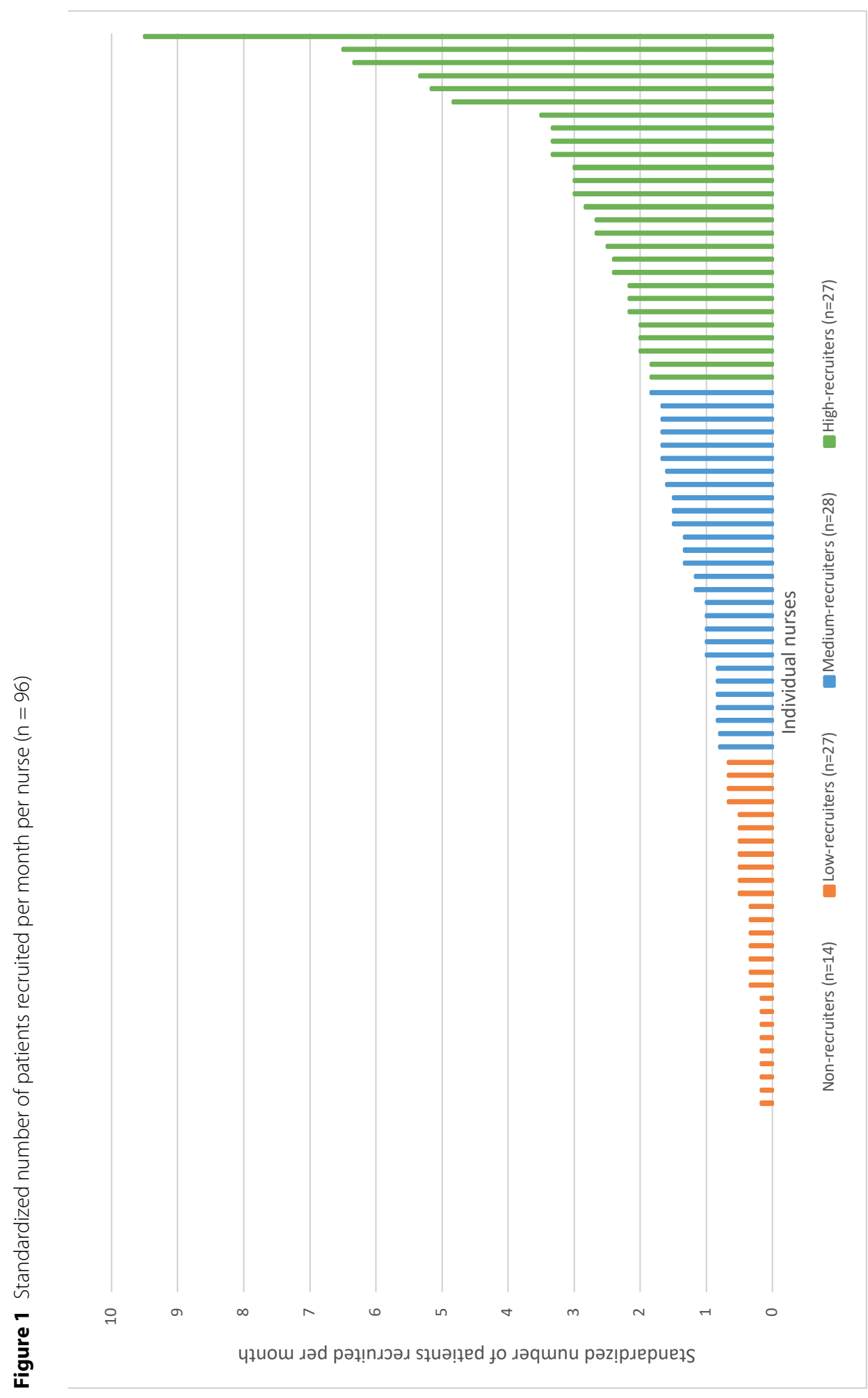




\section{Temporal shifts in recruitment success}

Figure 2 displays the absolute number of patients recruited per two-month period, only for those nurses who participated for the entire recruitment period. These data show a high early recruitment success that progressively declined over time. Of the 538 patients recruited by these nurses, 56\% ( $n=302)$ was recruited in the first two months. Within the third and fourth month this number declined to $32 \%(n=174)$ and in the last two months to $12 \%(n=62)$. Of the 74 nurses, fifteen did not recruit any patients within the first two months, of which nine (12\%) did not recruit any patients throughout the entire recruitment period. Nurses without recruitment success in the first two months recruited 17 patients in total, accounting for 3\% of the total.

It seems that low-recruiters mainly recruited their limited number of patients in the first two months, followed by a progressive decline in recruitment success afterwards. Mediumrecruiters largely recruited their patients in the first four months. Most high-recruiters remained successful throughout the entire recruitment period. 


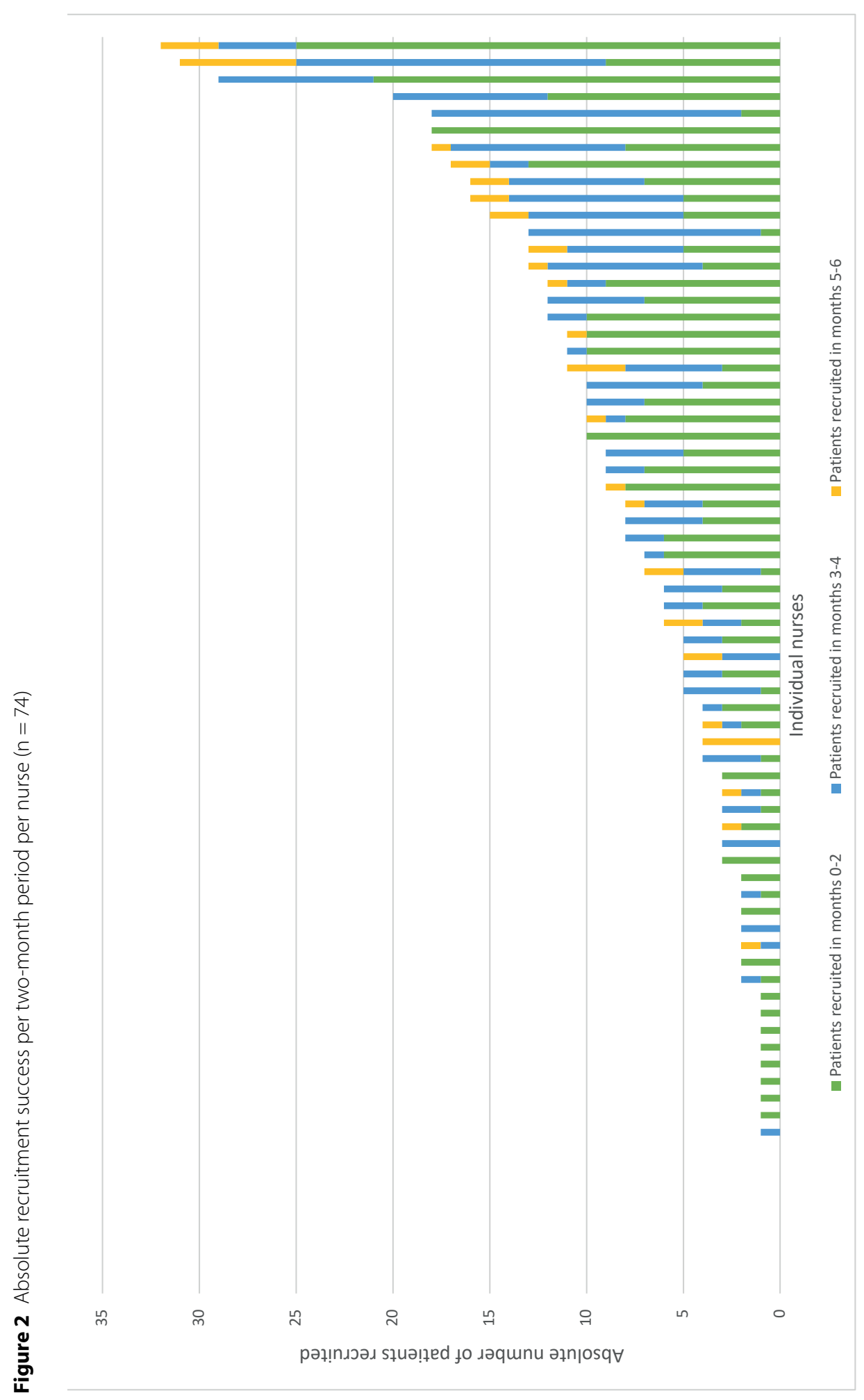




\section{Results of the interviews}

Sample characteristics of the interviewed nurses are presented in table 1. Most nurses were female $(N=14)$, reported extensive working experience, and were employed as practice nurses working in primary care $(\mathrm{N}=11)$. Diabetes nurses, working in secondary care $(\mathrm{N}=5)$, only provided healthcare to patients with diabetes.

Table 1 Sample characteristics of interviewees $(n=16)$

\begin{tabular}{lll}
\hline & N (\%) & Mean (SD) \\
\hline Gender & $2(12)$ & \\
Male & $14(88)$ & \\
Female & & \\
Nurse type & $11(69)$ & \\
$\quad$ Practice nurse & $5(31)$ & \\
Diabetes nurse & & \\
Patient population & $5(31)$ & $9.9(9.7)$ \\
$\quad \begin{array}{l}\text { Diabetes type 1 and/or 2 } \\
\text { Chronic patients }\end{array}$ & $11(69)$ \\
Age & & $24.3(6.7)$ \\
Nurse working years & & \\
Weekly consultation hours & & \\
\hline
\end{tabular}

a Including patients with diabetes, chronic obstructive pulmonary disease, asthma, and cardiovascular risk management

\section{Organizational factors}

Most nurses were experienced in recruiting patients to research. Approximately half of all nurses engaged in parallel research activities during our trial, but the majority reported activities that were unrelated to our trial population. In contrast to high-recruiters, some non-recruiters mentioned that research involving digital interventions neither seemed to be a relevant nor an urgent topic within their organizations as they described their patient population as largely non-digital oriented [Quote_\#1, Quote_\#2] (table 2).

Time and work pressure were expressed by almost all non-, low- and medium-recruiters and only sporadically by high-recruiters as barriers to recruitment efforts. All nurses, but especially non-recruiters, described their overall patient population as not eligible to participate; patients were commonly characterized as being too old and not digitally oriented [Quote_\#3]. 


\section{Study factors}

The study design and procedure were insufficiently clear to most non- and low-recruiters, reflected by little knowledge regarding the eligibility criteria, the program's content, the study design, and the randomization procedure. Contrary, medium- and high-recruiters provided a highly detailed description of the program, its content, and the randomization procedure. Nurses from all groups were pleased with the available support from the research team. Periodic email reminders were valued as helpful and necessary to stay alert.

\section{Recruiter factors \\ Motives to sign-up}

One non-recruiter stated that the decision to participate in the study was made by her physician [Quote_\#4]. Near all nurses of all groups expressed their willingness to participate, which was largely justified by positive attitudes such as the importance of research and the novelty of the innovation. Generally, low- to high-recruiters argued that the program could potentially yield personal benefits for their patients and themselves [Quote_\#5]. Additionally, high-recruiters labelled participation as fitting in their organization's vision and mentioned that eHealth could serve as a medium to support self-management initiatives.

\section{Implementation of recruitment}

All nurses initially approached their patients through consultations sessions. Most non- and low-recruiters shaped their efforts by expressing their involvement in research without extensively informing and persuading their patients to participate. Furthermore, most lowrecruiters mentioned to execute recruitment efforts without a concrete plan [Quote_\#6]. Contrary, medium- and high-recruiters indicated to have made efforts to persuade patients to participate by providing information, offering support, being enthusiastic, answering patients' questions, expressing conviction of the program's importance, and addressing positive attributes of eHealth and the program [Quote_\#7]. High-recruiters stated that providing study information and persuading patients took 5-1 minutes.

All nurses except high-recruiters stated to prioritize standard discussion topics within their consultations, such as the patient's well-being and glycemic control, over introducing patients to the study [Quote_\#8]. These nurses reported to value the program as less important because it did not yield immediate benefits for themselves or their patients. In addition, some nurses were reluctant to invest in potential future health improvements related to the program, as they were unsure about its outcome. Further, non- and low-recruiters expressed, in the view of the research team, unrealistic expectations of the efforts needed and the feasibility of the recruitment process as potential explanatory factors for their poor success.

The magnitude of patients declining the invitation to participate evoked negative emotional and practical responses in most non- and low-recruiters and some medium-recruiters. Examples of negative emotions entailed guilt for not recruiting patients, disappointment, and 
discouragement, while in practice this resulted in shifting study recruitment to a lower priority and forgetting recruitment [Quote_\#9, Quote_\#10]. By contrast, high-recruiters emphatically expressed being unaffected by patient rejections as they perceived it completely normal if patients would decline participation.

\section{Course of recruitment}

All non- and low-recruiters and most medium- and high-recruiters forgot patient recruitment at a certain point. Additionally, non- and low-recruiters indicated often not to manage recruiting patients or that the study became of inferior priority [Quote_\#11]. In most non- and low-recruiters, this process of forgetting and not managing recruitment seemed irreversible, i.e. at a certain point they invited no more patients to participate. A lack of time to extensively explain study details and persuade patients were frequently mentioned reasons for forgetting and not managing recruitment [Quote_\#12]. One medium-recruiter described the recruitment process as fluctuating, i.e. if patients accepted participation, alertness and motivation to recruit would increase, if patients declined participation, alertness would decrease [Quote_\#13].

One medium-recruiter coped with forgetting recruitment in consultations by telephoning patients afterwards. High-recruiters expressed their awareness of forgetting recruitment and coped with this by applying alternative recruitment strategies. For example, two nurses complemented consultation recruitment with telephone calls while another applied telephone calls and email contact instead, because of time constraints during consultations [Quote_\#14]. Although telephone recruitment was considered practical, most high-recruiters preferred face-to-face recruitment given the possibility to personally persuade patients.

\section{Facilitation of recruitment}

Half of the non- and low-recruiters, and all medium- and high-recruiter set a target regarding the number of patients they aimed to recruit, which in all cases was higher than the study target set by the research team. In addition, most nurses applied self-initiated reminders to facilitate their recruitment process. Some non-recruiters and almost all other recruiters indicated they had put their received instruction materials on their desk as a visual cue. One non-recruiter wrote a reminder note in her agenda, while similar notes were applied by many medium- and high-recruiters [Quote_\#15]. A shortcut to the program website was made by a few low-recruiters, while most high-recruiters opened the program website prior to their consultations. Checking patients' eligibility on appointment lists prior to consultations was frequently applied by medium- and high-recruiters and infrequently by low-recruiters. Additionally, a few high-recruiters put up a poster in their practice to raise study awareness and cognitive processing of patients.

\section{Patient factors}

Non- and low-recruiters stated that all or the majority of their patients declined study participation, while this occurred to a lesser extent in medium- and high-recruiters. Motivational 
issues such as no interest or not seeing the purpose of participation, and participation being considered confronting to their disease, were by all nurses considered reasons of patients not to participate. Patient barriers to participating were having no time or priority, and insufficient digital skills [Quote_\#16].

Generally, nurses characterised most patients who agreed to participate as intrinsically enthusiastic and open to digital innovations. Medium- and high-recruiters stated to have effectively persuaded initially reluctant patients [Quote_\#17]. 


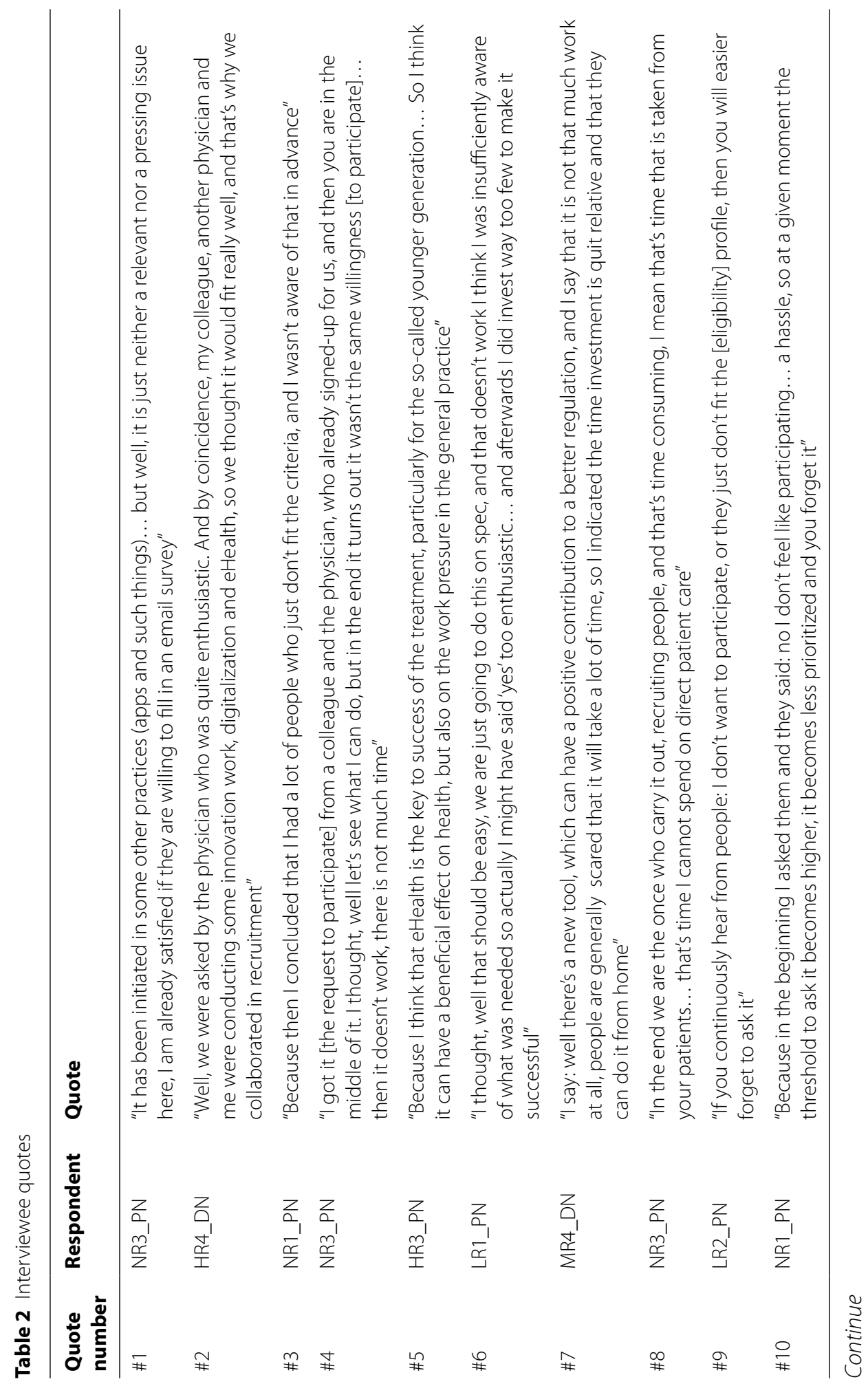




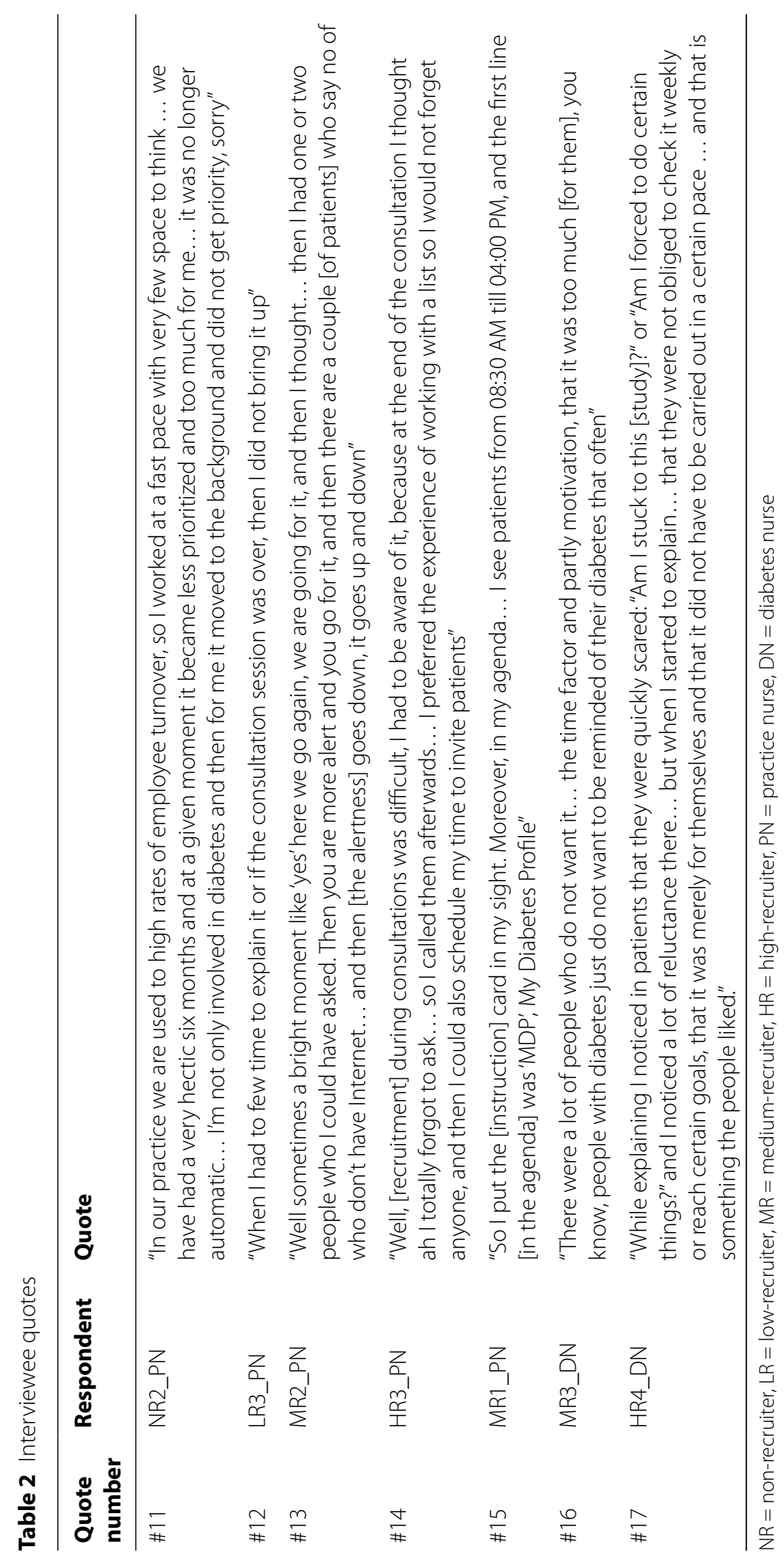




\section{Conclusions}

This study aimed to 1) describe nurses' recruitment success and its potential temporal shifts from a recent trial and 2) explore factors influencing recruitment success as perceived by the nurses themselves.

In our study $15 \%$ of the nurses were non-recruiters which was lower than the $25 \%$ reported in other studies. However, in these studies recruitment was performed by physicians $(89,90$, 172). Although nurses are considered to play a key role in recruiting patients to clinical trials, figures on nurses' recruitment success seem to be lacking, which hampers clear comparisons (178). A study comparing recruitment success of nurses to physicians indicated that nurses were as effective and more cost-effective recruiters, and suggested a more explicit role of nurses in patient recruitment (179). Compared to existing figures of physician recruitment, our nurses seem to be somewhat more successful in recruiting patients, but further comparative research is required.

Most T2DM patients visit their nurse every 3-6 months (14); our six-month recruitment period therefore provided sufficient opportunity for patients to be exposed to their nurses' recruitment efforts. Periodic reminders, available support from the research team, and the brief recruitment procedure may also have contributed to a lower non-recruiter percentage. A similar trial on an intervention to improve medication adherence also showed that about one-third of the physicians reached their set patient target, and that longer time to enroll the first patient was associated with poor recruitment success (172). Initial recruitment failure negatively affects recruiters' motivation and beliefs about the study's feasibility, which in turn could slow down or prematurely terminate recruitment efforts (180). In that light, recruiters should aim for early success to maintain motivation and sustain study efficacy beliefs.

From the interview data, we observed a clear dichotomy between non/low-recruiters compared to medium/high-recruiters regarding factors influencing recruitment success.

\section{Recruitment process of non- and low-recruiters}

Patient recruitment of non/low-recruiters appeared to be similar. These nurses showed poor study, program, and procedure-specific knowledge, possibly resulting in insufficient informing and persuading their patients to participate. Generally, all nurses in our trial characterized their general patient population as both ineligible and unwilling to participate in our study; previously also a reported barrier for recruitment success $(90,172)$. Therefore, a lack of information and persuasion may explain that patients of non/low-recruiters generally rejected participation. Socio-cognitive models posit that patients' willingness to participate could be improved by transferring study and program knowledge and exploring benefits to initiate a patient's cognitive processing of the decision to participate in a trial $(37,38)$. Furthermore, non/low-recruiters might benefit from learning coping skills on how to respond 
to patient rejections, given their subsequent lack of recruitment effort (180). Especially for professionals with little practical recruiting experience, it might be beneficial to apply guided practice in which they are offered demonstrations and training on how to address the study in consultations, and empowered to rehearse recruitment behaviour (181).

Non/low-recruiters viewed the study as less important compared to regular healthcare work and as not immediately rewarding, which emerged as recruitment barriers earlier $(94,180)$. Additionally, nurses' time and work pressure were mainly brought-up as barriers to introduce the study to patients. Competing demands and time pressure are also commonly reported barriers to recruitment success, and hamper fitting research into daily practice $(173,180,182$, 183). Hence, personal benefits and barrier-focused coping strategies need to be incorporated in recruitment enhancing interventions.

\section{Differences between non- and low-recruiters}

A non-digital oriented working environment and unrealistic effort and feasibility expectations may have contributed negatively to recruitment success in non-recruiters. A lack of empowerment due to practice policy or culture and recruitment efforts taking more time than expected, also emerged as barriers elsewhere (172). Despite acknowledging the value of research, which generally substantiates initial participation (180), no personal benefits for signing-up were brought-up by non-recruiters contrary to low-recruiters, which may explain low-recruiters' slightly higher success $(38,184,185)$.

\section{Recruitment process of medium- and high-recruiters}

The recruitment process of medium- and high-recruiters also showed similarities. These nurses expressed various personal benefits of signing-up, substantiating their willingness to participate (38). Contrary to non/low-recruiters, these nurses expressed their enthusiasm in making efforts to inform and persuade patients to participate, which contributes to successful trial recruitment (89). Together with more extensive study knowledge, their enthusiasm may have positively impacted confidence and willingness to recruit patients (180).

High- and some medium-recruiters applied alternative recruitment strategies to cope with forgetfulness and to overcome time constraints. Recruitment during daily care may be difficult if standard discussion topics require additional time, leaving insufficient room for introducing a study (180). Substituting recruitment during consultations by more suitable strategies in case of time constraints, such as email or telephone recruitment are important to increase the likelihood of successful recruitment efforts (47). Moreover, medium/high-recruiters frequently applied self-initiated and research team reminders which may have facilitated recruitment efforts, alertness in response to anticipated forgetfulness, and overcoming barriers (186). 


\section{Differences between medium- and high-recruiters}

High-recruiters perceived trial participation as congruent to their organization's vision; favourable organizational circumstances contributed to fruitful recruitment earlier (174). Further, high-recruiters did not prioritize standard discussion topics over suggesting participation, which may have resulted in discussing the study more frequently. Additionally, high-recruiters remained consistent recruiters throughout the entire recruitment period, contrary to medium-recruiters whose success decreased in the final recruitment months. Last, self-initiated reminders were more often employed by high-recruiters.

\section{Strengths and limitations}

First, we provided a thorough evaluation of factors affecting recruitment success. Second, perspectives of recruiters were examined, rather than findings gathered from reviews or perceptions from research teams. This study only focused on the initial enrolment of patients rather than their final decision whether or not to participate or their retention in the trial, which can be regarded as a limitation. Future studies should examine if e.g. high recruitment success results in corresponding final consent and retention rates, which is primarily important when considering sufficient study power. Last, observational research designs are required to possibly invigorate current findings statistically.

\section{Implications and conclusion}

First, if feasible, researchers should continue including recruiters after initial recruitment onset, as we showed that $20 \%$ of the patients were enrolled by later entrants. Second, early recruitment success is encouraged as initial failure often determines poor recruitment. Third, patient recruitment success seems to be partly influenced by an integration of recruiter factors such as knowledge, motivation, intention and self-regulatory capacities (36-38, 43, 47, 56). Although other factors may provide relevant intervention targets to improve success, recruiter factors may be the most viable and changeable domain to intervene in (33).

The expressed intention by professionals to recruit patients is generally the result of information provided by the research team and subsequently the motivation of the professional (43). Whilst nurses are recruited, they should primarily be informed and persuaded on the study's content, procedure, feasibility, and benefits to substantiate and enhance their willingness to participate. Further they should be made aware that patient recruitment will require an investment in short term, i.e. informing and persuading patients, to increase the likelihood of generally unwilling patients to participate.

Sufficient motivation does not automatically warrant recruitment enactment and success $(47,56,89)$. Hence, recruiters should be prepared, skilled and confident to optimally deliver recruitment efforts, in terms of when, where and how to act, and how to cope with barriers such as patient rejections, time constraints and forgetfulness (187). Reminders may facilitate the likelihood of successful translation of intentions towards recruitment efforts. However, 
these may only suit recruiters who have a high intention towards patient recruitment, unintentionally forget recruitment or perceive recruitment barriers such as time pressure, contrary to nurses who deliberately terminate recruitment $(186,188)$. Nurses who deliberately slow-down or terminate recruitment, or nurses without recruitment success yet, should primarily be approached by informative and motivational messages rather than barrierfocused coping messages or reminders. 


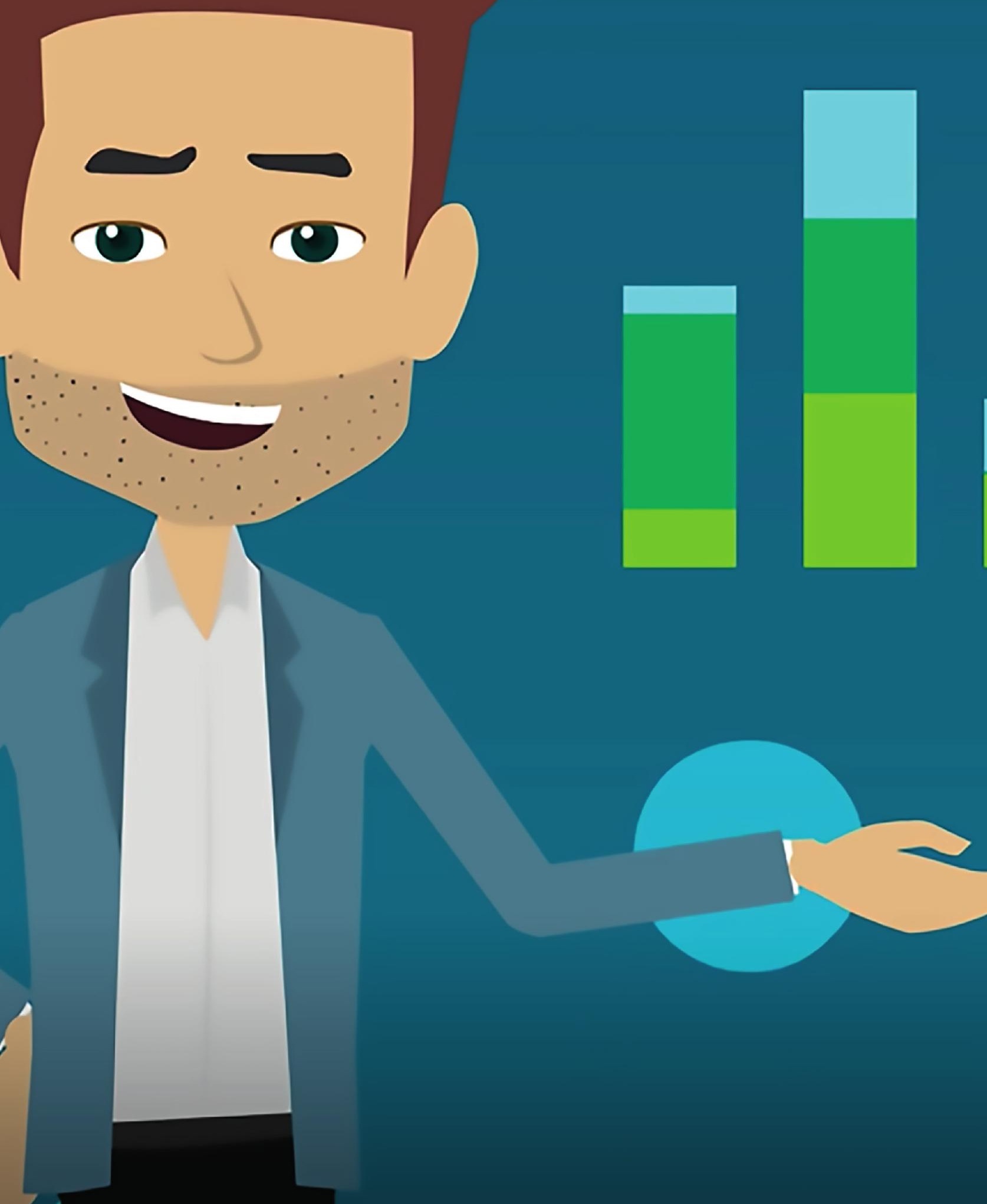




\section{Chapter 6}

The effectiveness of a web-based computer-tailored program to improve treatment adherence in patients with type 2 diabetes: A randomized controlled trial

This chapter is submitted as:

Vluggen S, Hoving C, Candel, M., Schaper NC, de Vries H. The effectiveness of a web-based computer-tailored program to improve treatment adherence in patients with type 2 diabetes: A randomized controlled trial 


\section{Abstract}

Background and objectives: Adherence to core type 2 diabetes mellitus (T2DM) treatment behaviors is suboptimal and non-adherence is generally not limited to one treatment behavior. The Internet holds promise for programs which aim to improve adherence. We therefore developed an eHealth program for patients with T2DM to improve their overall treatment adherence, i.e. adherence to both healthy lifestyle and medical behaviors, and tested its effectiveness in a randomized controlled trial.

Methods and materials: Patients with T2DM were recruited by their health professional and randomized into either the intervention arm (i.e. access to the eHealth program for six months; $n=243$ ) or a waiting-list control arm ( $n=244)$. Outcome data was collected through online assessments on physical activity levels, caloric intake from unhealthy snacks and adherence to oral hypoglycemic agents and insulin therapy. Changes to separate behaviors were standardized and summed into a composite change score representing changes in overall adherence. Further standardizing these composite change scores yielded the primary outcome. Standardized change scores observed in separate behaviors acted as secondary outcomes. Mixed linear regression analyses were conducted to examine the effectiveness of the intervention on overall and separate treatment behavior adherence, accommodating for relevant covariates and patients nesting. The standardizations applied in the primary and secondary outcomes allowed for interpreting the regression coefficient of the treatment in the analyses as effect sizes.

Results: After the six-month follow-up assessment, 111 patients in the intervention arm (47\%) and 177 patients in the control arm (73\%) were retained. After controlling for relevant confounders, overall treatment adherence improved significantly in the intervention arm, compared to the control arm, reflected by an overall small to medium effect size $(d=.24, p=.05)$. In comparison to the control arm, a significant decrease was observed in caloric intake from unhealthy snacks only, when considering changes in separate treatment behaviors $(d=.38 ; p=.01)$.

Conclusions: Our multi-behavior program significantly improved overall adherence to core T2DM treatment behaviors compared to the control arm. To further enhance the intervention's impact, a wide-scale implementation of our eHealth intervention is suggested in order to be of impact in the personal, societal and economic area. 


\section{Introduction}

Worldwide, 425 million people aged 20-79 years old live with Type 2 Diabetes Mellitus (T2DM) with expectations of over 600 million people being affected by 2045 (1). T2DM is associated with considerable morbidity and mortality rates, which reduce the patients' quality of life and life expectancy, and pose an enormous economical and societal burden (1, 7). Guidelines recommend a series of core treatment behaviors for patients with T2DM. These include more healthy lifestyles, i.e. improving dietary patterns and increasing physical activity (PA), and, if applicable, adequate adherence to medical strategies such as oral hypoglycemic agents (OHAs) and/or insulin therapy $(14,15)$. Despite the chronic progressive nature of T2DM, patients who adhere to these behaviors can live long and high quality lives (1).

Unfortunately, patients' adherence to separate behaviors is inadequate; dietary and PA targets are not met consistently, and most studies on adherence to medical strategies report adherence prevalence percentages below $80 \%$, which is generally considered insufficient adherence $(23-25,29,30)$. Moreover, less than $5 \%$ of the patients diagnosed with T2DM adheres to all treatment behaviors while over $80 \%$ could either improve two or more (25). Poor adherence can result in suboptimal clinical treatment benefit, such as disease worsening, an increase in comorbidity, a reduction of patients' quality of life, increased healthcare expenditures and hospitalizations, and early mortality $(7,16-22)$.

Non-adherence is a complex process and is a result of an interaction of multiple factors including social and economic factors, the healthcare system, characteristics of the disease and therapy, and patient-related factors $(32,33)$. Although all these factors provide relevant entries for targeting non-adherence, most are difficult to change, while if changed, they may only affect adherence improvements indirectly through patient factors (33). However, patientrelated factors themselves (e.g. awareness, beliefs, motivation and self-regulatory capacities) have shown to be relatively changeable and have a direct impact on treatment adherence (84). Hence, these determinants need to be addressed in interventions when aiming for improvements in treatment adherence.

Several patient-focused interventions already exist that aim to improve treatment adherence; the majority focusses on improvements in adherence to blood glucose lowering medication $(65,66,189)$, resulting in no to small effects on glycemic control. Glycemic control is however not only the result of medication adherence but also greatly affected by (un)healthy lifestyle behaviors $(33,68)$. Therefore, interventions that target a combination of both healthy lifestyle and medical behaviors, i.e. a multi-behavior approach, might be more likely to be effective (25).

Besides such a multi-behavior approach, other factors may also enhance the effectiveness of interventions that aim to improve adherence. The Internet holds promise for a wide scale promotion of behavioral change to facilitate the management of $\operatorname{T2DM}(65,70,71)$. Internet 
interventions as a delivery platform for health promotion and health services activities, also referred to as eHealth, have shown to be (cost)-effective, easy in use, have fewer availability restrictions than regular medical consultations, and can temper pressure on healthcare systems $(69,73-78)$. A more advanced eHealth strategy applies computer-tailored technology; a strategy that provides patients with tailored content based on unique answers given to a web-based assessment (81). Further success factors of eHealth interventions include the application of a theoretical foundation, provision of interactive tailored content, application of goal-setting strategies and monitoring tools, identification of risk behaviors, using visually supported content and focusing on distinct behavior change phases, i.e. awareness, motivation, and self-regulation $(65,70,71,79,80)$.

Yet, a recent review on eHealth interventions supporting T2DM management (71) concluded that only one of the nine included studies reported significant improvements in dietary behavior and PA (79). Generally, such eHealth interventions, often include little interactive content and tailored strategies, are mainly text-based, make little use of theoretical foundations and technology, and focus on separate behaviors that play a role in the management of T2DM instead of combining behaviors $(65,70,71,80)$, which may explain the relative poor results of available interventions.

Hence, eHealth interventions aimed at T2DM treatment adherence might be significantly improved by building on a theoretical base, incorporating computer-tailoring technology, providing interactive and visually supported content, and applying a multi-behavior approach.

We therefore developed an eHealth program for patients with T2DM including the abovementioned success factors in order to improve treatment adherence to core T2DM treatment behaviors, i.e. healthy lifestyle and medical behaviors. The main aim of this study was to examine the effectiveness of this program on overall treatment adherence in a randomized controlled trial (RCT). In addition, we examined changes to separate treatment behaviors as a result of the program.

\section{Material and methods}

\section{Study design}

We conducted an RCT including an intervention arm and waiting-list control arm to examine the effectiveness of a novel eHealth program, 'My Diabetes Profile' (MDP), on treatment behavior adherence in patients with T2DM. A more extended description of the program, including its development and content, as well as a trial protocol is available elsewhere (175).The study was evaluated and approved by the Medical Ethics Committee of Maastricht University Medical Center (16-4-171). The committee concluded that no ethical clearance 
was needed according to the rules and regulations of the Medical Research Involving Human Subjects Act (WMO). The trial is registered in the Dutch Trial Register (NTR6840).

\section{My Diabetes Profile program}

The MDP-program aims to improve patient adherence to core T2DM treatment behaviors. This implies improving PA-levels, decreasing caloric intake from unhealthy snacks - as this emerged as a major issue in T2DM patients' diet in an earlier study (28) - and increasing adherence to medical strategies, i.e. oral hypoglycemic agents and/or insulin therapy $(14,15)$.

The MDP-program is theoretically grounded in the I-Change Model (43) which integrates various acknowledged socio-cognitive theories that assume a deliberate process when someone engages in (health) behavior $(36-38,56)$. The model has frequently been applied to map salient socio-cognitive determinants of health behavior and to develop effective webbased computer-tailored interventions aimed at health behavior change accordingly $(51,52)$. The MDP-program is divided in two nearly identical blocks, each available to users for three months. Each block consists of three sessions, applying both assessment-based tailored video and text feedback: 1) health risk appraisal, 2) awareness and motivation, and 3) goal-setting, action planning and self-regulation.

The health risk appraisal session provides patients with interactive and tailored content on their risk behaviors. Primarily, adherence levels are assessed for all behaviors the patient was involved in. For behaviors subject to improvement, the participants' intention to change that behavior is assessed. The final part of the first session enables patients to select a single improvable behavior which will be their focus for the following three months while working with the MDP-program. In the event of meeting all guideline targets, patients are prompted to select PA as Dutch guidelines recommend any PA beyond the minimum weekly standard of 150 minutes (158). A patient who selects a behavior that is accompanied by a low intention to change, is navigated to the awareness and motivation session. This second session aims to raise patients' awareness for the need to improve the particular behavior and to increase motivation, with the ultimate purpose to achieve a high intention to change. If a high intention to change is achieved, after either session one or session two, the patient is directed to session three on goal-setting, action planning and self-regulation. This session aims to increase the likelihood of successful translation of the expressed intention into subsequent behavior. This process is facilitated by setting small and realistic goals, forming action plans on where, when and how to perform the behavior, and forming self-regulation strategies to cope with barriers or situations with may impede adherence.

\section{Participants and procedure}

Participants were recruited to the program by their practice nurse or diabetes nurse. In the Netherlands, patients with T2DM are predominantly treated by a practice nurse or diabetes nurse, in the general practice and outpatient hospital setting respectively, and are supervised 
by a physician (14). Patients usually visit their nurse every three to six months (14). Therefore, these nurses were considered to be in an ideal position to recruit patients for this trial. Nationwide, nurses were approached via email, telephone calls, letters to their work address, and social media platforms (i.e. Linkedln and Facebook). They could sign up for the study by contacting the research team directly, or by registering via the project website. Nurses were asked to recruit at least eight patients within a period of six months. Inclusion criteria for patients comprised of: 1) T2DM diagnosis for at least one year, 2) being 40-70 years old, 3) using at least one form of oral blood glucose lowering drugs or insulin, and 4) having no walking disability. Exclusion criteria comprised of: 1) not speaking or understanding the Dutch language, 2) having no access to the Internet, and 3) using an insulin pump.

After obtaining information on the study and upon expressing interest, eligible patients were recruited to the program by their nurse by completing a brief online registration form. Data input consisted of the participant's name, telephone number (optional), birth date, most recent $\mathrm{HbA}_{1 c}$-level (a measure for glycemic control), the year of diabetes diagnosis, current diabetes medical strategy, and e-mail address. Once registered, patients received an e-mail including log-in data which primarily provided access to additional study and procedure information before providing informed consent. Participants would then fill in the baseline questionnaire after which they were randomly allocated to either the intervention arm (receiving program access for six months), or the control arm (receiving care as usual). Randomization occurred at the individual level by means of computer software randomization.

Individuals allocated to the intervention arm were given access to assessment-based videoand text-tailored feedback of the MDP-program for six-months. Individuals allocated to the control arm were informed about their group allocation after baseline completion and notified that they would be invited for the follow-up questionnaire six months later. Individuals in the control arm were given the opportunity to access the MDP-program after completion of the follow-up assessment as part of a waiting-list control principle. Information regarding delayed program access for participants who completed the control arm was provided as part of the additional study information given prior to consenting.

\section{Measures}

The baseline questionnaire included 131 questions on demographic characteristics, comorbidities, smoking status, and current PA-levels, caloric intake from unhealthy snacks, and adherence to oral hypoglycemic agents and/or insulin therapy. Questionnaires were identical for both trial arms.

\section{Demographic characteristics}

Demographics assessed only at baseline included the participant's gender, age, education level, body length, and nationality. Living arrangement, net income, work status, T2DM 
medication type, and body weight were assessed both at baseline and follow-up. Body Mass Index (BMI) was calculated as weight/length².

\section{Comorbidities}

Questions on comorbidity assessed whether participants ever suffered in the past, or currently suffered from several conditions including depression, stroke, heart failure, myocardial infarction, cancer, COPD/asthma/bronchitis, rheumatoid arthritis/osteoarthritis, and Crohn's disease. Comorbidities were assessed at baseline only.

\section{Physical activity}

PA-levels were assessed using the validated Short Questionnaire to Assess Health-Enhancing Physical Activity (SQUASH) (166). The SQUASH assesses various domains of PA, for which the average daily hours and minutes, and the number of days per week activities are carried out, are reported. Each domain corresponds with a specific metabolic equivalent of task (MET) value, that is, an intensity and energy expenditure ratio of a task compared to energy expenditure while in rest (190). As national guideline targets recommend physical activities $\geq 3 \mathrm{MET}$, and because the SQUASH includes two activities $<3$ MET, these activities were excluded (191). A cumulative number of weekly $\geq 3$ MET PA minutes was calculated accordingly.

\section{Unhealthy snack intake}

Weekly caloric unhealthy snack intake was assessed by a self-administered food frequency questionnaire (FFQ). The FFQ includes unhealthy snacks identified by earlier studies, complemented with snacks commonly consumed in the Netherlands $(167,168)$. The unhealthy snacks listed in the FFQ are translated into a particular amount of calories consumed, based on the calorie data-base of the Dutch nutrition center (192). A total of weekly caloric intake was calculated, based on the participant's intake from unhealthy snacks.

\section{Oral hypoglycemic agent adherence}

Oral drug adherence was measured through the Probabilistic Medication Adherence Scale (ProMAS) questionnaire (124). The scale includes eighteen items which assess a variety of adherence behaviors, such as, forgetting, stopping, changing dosages, and taking more or less medication than prescribed. To reduce potential recall bias, a time period of three months was added to every item (122). A sum score was calculated for the eighteen items, ranging from 0-18, with higher scores representing better adherence.

\section{Insulin therapy adherence}

Insulin therapy adherence was assessed through an adapted version of the ProMAS questionnaire and included nine items which were assessed over a three-month time period. Non-relevant items, i.e. items that did not distinguish between adherence and nonadherence for insulin therapy were removed. For instance, the item 'I sometimes take fewer medicines than prescribed by my doctor' was removed from the original questionnaire as 
patients using insulin should adjust their dosage in case of hypoglycemia or illness. A sum score was calculated for the nine items, ranging from 0-9, with higher scores representing better adherence.

\section{Primary outcome measure, primary end point, and sample size calculation}

The primary outcome measure was a composition score of changes in separate treatment behaviors addressed in the program. To create such a composition score, changes in each treatment behavior, i.e. changes in PA-levels, caloric intake from unhealthy snacks, and $\mathrm{OHA}$ and insulin therapy adherence, were standardized into separate change scores. For each treatment behavior in each participant, baseline scores were subtracted from follow-up scores, yielding a change score. The change score for caloric intake was reversed as the MDPprogram aimed to decrease caloric intake from unhealthy snacks. In order to standardize the outcomes of the different behaviors, the change score of each participant was divided by the pooled standard deviation of the change scores of this specific behavior (165). The pooled standard deviation of the change scores of both trial arms was used. Finally, these separate standardized change scores were then per participant summed into a composite change score (193).

The composite change score was transformed, i.e. standardized further by dividing the composite change scores for each participant by the pooled standard deviation of these composite change scores. Again, the pooled standard deviation of the composite change scores of both trial arms was used. Afterward, a mean of these'standardized composite change scores' could be calculated per trial arm. The difference in these means gives information about the direction as well as the magnitude of the difference between groups, in that it can be interpreted as Cohen's $d$ (effect size, ES) for the difference between treatment and control on the variable 'overall change in treatment adherence' $(193,194)$. The standardized composite change sore is the primary outcome measure and Cohen's $d$ is the primary end point of this study.

The power calculation was based on the primary outcome measure. We aimed to detect a difference of the mean of the primary outcome measure $(=d)$ between both trial arms of 0.4 in a two-tailed test at a $5 \%$ type I error rate $(194,195)$. Considering an intraclass correlation coefficient of 0.02 and a statistical power of $80 \%, 116$ participants per trial arm would be sufficient at the trial's follow-up assessment (132). Given an expected attrition rate of 50\%, we aimed to include 464 participants with a completed baseline assessment.

\section{Secondary outcomes: changes in separate treatment behaviors}

Besides the calculation of a standardized composite change score, also separate changes in PA-levels, caloric intake by unhealthy snacks, and OHA and insulin therapy adherence were calculated. A standardized change score per treatment behavior was calculated per 
participant by subtracting the baseline score from the follow-up score, yielding a change score. Subsequently, this change score of each participant was divided by the pooled standard deviation of the change scores of this specific behavior, as described above (165). The difference of the means of these standardized change scores between the intervention and control arm can again be interpreted as Cohen's $d$ but now for a specific treatment behavior (194).

\section{Statistical Analyses}

All analyses were conducted using SPSS version 24.0, using a 5\% significance level. Frequency and descriptive analyses were used to describe sample characteristics. Independent-samples $t$ tests and $\mathrm{Chi}^{2}$-tests were conducted to identify significant differences at baseline between the intervention and control arm participants regarding demographics and separate treatment behavior scores. Through logistic regression, selective attrition after randomization regarding demographics was examined.

As participants were nested within nurses participating in the trial, mixed linear regression analyses were conducted to assess the effectiveness of the MDP-program on the primary and secondary outcome measures. Covariates in the model were included based on the assumptions of the theoretical framework that was applied in this study, i.e. the I-Change Model, that is gender, education level, net income, living arrangement, work status, BMI, $\mathrm{HbA}_{1 \mathrm{c}}$-level, T2DM medication type, recruitment nurse type, and depression status (196). Nonsignificant covariates were stepwise eliminated applying a backward elimination procedure, implying that at each step the least (and non-) significant covariate was removed from the model. This process was repeated until only significant covariates remained in the regression model (136). The results of the starting model before backward elimination as well as the final model will be presented for the primary outcome measure. For secondary outcome measures only the final model will be presented. Because of the standardizations involved in the primary and secondary outcome measures, the regression coefficient of the treatment factor in the mixed regression analysis can be interpreted as Cohen's $d$, whether or not adjusted, depending on whether or not there are covariates in the model.

To address attrition, sensitivity analyses were carried out. Sensitivity analyses comprised of a complete case scenario where all participants with complete data were included, and imputation scenarios also involving those participants of whom the primary outcome was not available. Four different imputation scenarios were performed: two optimistic and two pessimistic scenarios. In the optimistic scenarios we assumed that, compared to the complete case analysis, drop-outs improved in the primary outcome, while in the pessimistic scenarios we assumed that they deteriorated. For both the optimistic and pessimistic scenarios, two imputation variants were performed, as attrition was unequal in the arms of the trial, and this imbalance might affect the results of the analysis. In the equal variant, the imputed value was drawn for both the intervention and control arm assuming a normal distribution 
of the outcome with a mean equal to the condition mean $+/-1 x$ the standard deviation. In the unequal variant we used either a mean equal to the intervention mean $+/-1 \mathrm{x}$ the standard deviation for the intervention arm, and a mean equal to the control mean $+/-1.5 \mathrm{x}$ the standard deviation in control arm. This unequal variant reflects the possibility that the outcome on the average either improved or deteriorated to a lesser extent in the participants of the intervention arm, compared to the control arm. More effort was required from the participants of the intervention arm which may have affected attrition.

\section{Results}

\section{Sample characteristics}

Figure 1 displays the flow of participants throughout the trial from initial recruitment and registration by nurses to the completion of follow-up assessment and requested program access by control-arm participants. Of the 669 participants registered to the program, 508 (76\%) signed the online informed consent. Of these, 478 (94\%) completed the baseline assessment and were randomly allocated to either the intervention arm $(234 ; 49 \%)$ or control $\operatorname{arm}(244 ; 51 \%)$.

The baseline sample of participants (table 1) had a mean age of 60.2 years (range 40-70), one thirds was female (32.4\%), and 40.2\% was low-educated. Most participants lived together with a partner. A slight majority of the participants (62\%) used OHAs as the only medication to control their blood glucose levels, while either only insulin therapy or a combination of insulin with OHAs was used by approximately $7 \%$ and 32\%, respectively. Participants had on average an unfavorable $\mathrm{HbA}_{1 \mathrm{c}}$-level and BMI. At baseline, participants had a mean OHA adherence score of 13.2, which indicates medium-high adherence (124). The mean insulin therapy adherence score was 7.7, which implies high adherence. On weekly average, participants were physically active for 815 minutes and consumed 1710 calories from unhealthy snacks. Baseline characteristics of participants allocated to the intervention arm (MDP-participants) and control arm (control participants) were comparable, except for the participant's age which was approximately 1.5 years higher in the MDP-participants $(p=.01)$.

On average, patients could improve three treatment behaviors. At the first occasion to select a single treatment behavior, most MDP-participants chose to improve PA-levels ( $n=88,43 \%)$, followed by decreasing unhealthy snack intake $(n=66,33 \%)$, improving OHA use $(n=39$, $19 \%)$, and improving insulin therapy adherence $(n=10,5 \%)$. After three months, patients were prompted again to choose a single treatment behavior. On this occasion, again most patients chose to improve PA-levels ( $n=46,44 \%$, followed by decreasing unhealthy snack intake $(n=$ $39,38 \%)$, improving OHA use $(n=15,14 \%)$, and improving insulin therapy adherence $(n=4$, $4 \%$ ). Slightly over $40 \%$ of the patients chose the same behavior to improve on at the second occasion as in the first three months of the program. Three patients met all guideline targets, 
either on the first or second occasion, and were therefore prompted to select PA, as this was considered improvable regardless of the initial level.

\section{Attrition analyses}

Six months after completing the baseline assessment, 288/478 (60\%) participants completed the follow-up assessment, of which 111/234 (47\%) of the intervention arm and 177/244 (73\%) of the control arm (figure 1). Drop-outs either resigned participation actively or did not complete the follow-up assessment despite being reminded to do so. Of the 177 control participants who completed the follow-up assessment, about one-third requested program access, offered as part of the waiting-list control principle. Control participants were more likely to be retained for the study $(\mathrm{OR}=7.56,95 \% \mathrm{Cl}$ 2.79-20.48).

\section{Effect analyses on primary outcome measure (overall adherence)}

The results of the linear mixed regression analyses before and after the backward elimination procedure are shown in table 2 . Before backward elimination, results show that allocation to the MDP-program had a positive effect on standardized composite change score $(d=.38$; $95 \% \mathrm{Cl} .099$ to $.655 ; p=.01$ ). After stepwise backward elimination of insignificant covariates, allocation to the MDP-program also had a significant positive effect on overall treatment adherence $(d=.24 ; 95 \% \mathrm{Cl} .005$ to $.481 ; p=.046)$. This implies that the program had a significant small to medium effect on overall treatment adherence, compared to the control arm.

\section{Sensitivity analyses}

The results of the sensitivity analyses partially reflect the results of the effect analyses on complete cases as discussed in section 3.3. After replicating the final mixed linear regression analyses following an equal and unequal optimistic imputation scenario, the intervention effect remained significant both under the equal imputation $(d=.39 ; 95 \% \mathrm{Cl} .201$ to .579 ; $p=<.01)$, and under the unequal imputation $(d=.33 ; 95 \% \mathrm{Cl} .120$ to $.537 ; p=<.01)$. Following an equal and unequal pessimistic imputation scenario, the intervention effect disappeared under the equal ( $d=-.13 ; 95 \mathrm{Cl}-.353$ to $.090 ; p=.25)$ and unequal imputation $(d=-.04 ; 95 \% \mathrm{Cl}$ -.271 to $.189 ; p=.73)$.

\section{Effect analyses on secondary outcome measures (separate behavior adherence)}

Table 3 displays the raw mean adherence scores at baseline and follow-up per treatment behavior per trial arm. In addition, the corresponding intervention effect is displayed as Cohen's $d$, and included co-variates in the final model are shown in a footnote below the table. At follow-up, the mean OHA adherence score increased in both intervention and control arm participants, i.e. from 13.5 to 14.2 and from 13 to 13.4 respectively. The average insulin therapy adherence score also increased in both arms from 7.4 to 8.0 in the intervention arm and from 7.7 to 7.9 in the control arm. Weekly average PA-levels decreased in intervention arm participants from 865 to 833 minutes, while levels increased in control arm participants 
from 789 to 884 minutes. The mean weekly caloric intake from unhealthy snacks decreased in intervention arm participants from 1857 to 1269 calories while control arm participants decreased their intake from 1656 to 1496 calories. When compared to the control, the intervention had a significant effect on the decrease in caloric intake from unhealthy snacks, with a small to medium effect size $(d=.38 ; 95 \% \mathrm{Cl} .098$ to $.661 ; p=.01)$.

Figure 1 Participant and randomization flow throughout the trial

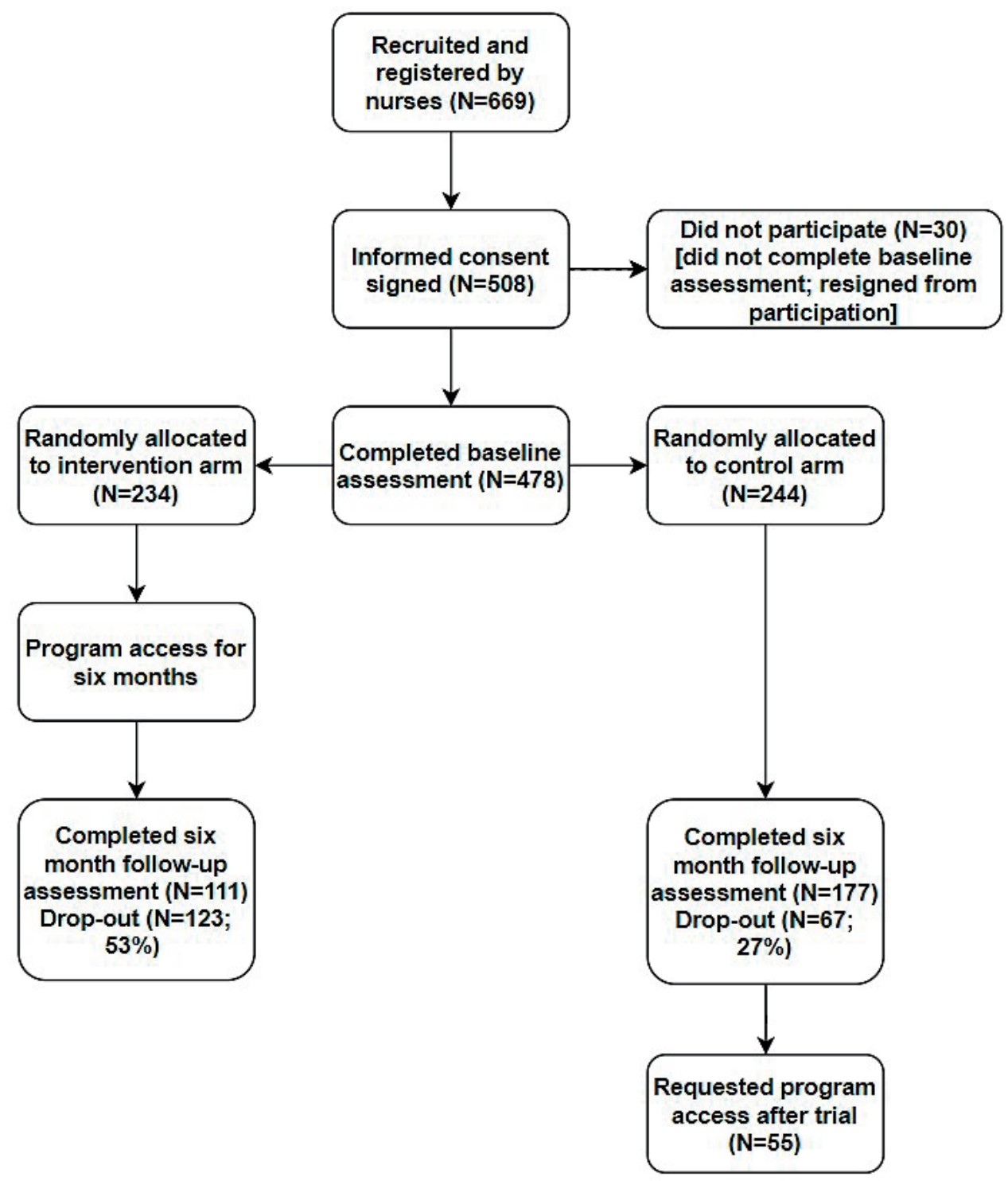


Table 1 Sample characteristics of patients at baseline and comparison of baseline characteristics between intervention arm and control arm

\begin{tabular}{|c|c|c|c|c|c|c|}
\hline Characteristics & $\begin{array}{l}\text { Overall } \\
\text { sample } \\
(\mathrm{N}=478)\end{array}$ & $\begin{array}{l}\text { Intervention } \\
\text { arm } \\
(\mathrm{N}=234)\end{array}$ & $\begin{array}{l}\text { Control } \\
\text { arm } \\
(\mathrm{N}=244)\end{array}$ & $x^{2}$ & T Value & $\begin{array}{l}P \\
\text { value }\end{array}$ \\
\hline Age in years, mean (SD) & $60.2(6.8)$ & $60.9(6.3)$ & $59.4(7.1)$ & & 2.46 & .01 \\
\hline Female, n (\%) & $155(32.4)$ & $76(32.5)$ & $79(32.4)$ & $<0.1$ & & .98 \\
\hline Education level, n (\%) & & & & 0.7 & & .69 \\
\hline Low & $192(40.2)$ & $90(38.5)$ & $102(41.8)$ & & & \\
\hline Middle & $108(22.6)$ & $56(23.9)$ & $52(21.3)$ & & & \\
\hline High & $171(35.8)$ & $85(26.3)$ & $86(35.2)$ & & & \\
\hline Living arrangement, n (\%) & & & & $<0.1$ & & .91 \\
\hline Together with partner & $370(77.1)$ & $182(77.8)$ & $188(77)$ & & & \\
\hline Alone & $107(22.3)$ & $52(22.2)$ & $55(22.5)$ & & & \\
\hline Work status, n (\%) & & & & 1.6 & & .67 \\
\hline Salaried/Self employed & $207(43.3)$ & $96(41.0)$ & $111(45.5)$ & & & \\
\hline No salaried employment & $74(15.5)$ & $35(15.0)$ & $39(16.0)$ & & & \\
\hline Retired & $145(30.3)$ & $75(32.1)$ & $70(28.7)$ & & & \\
\hline Disabled/Incapacitated & $52(10.9)$ & $28(12.0)$ & $24(9.8)$ & & & \\
\hline Net. Income, n (\%) & & & & 0.2 & & .64 \\
\hline Under average income & $130(27.2)$ & $60(25.6)$ & $70(28.7)$ & & & \\
\hline Above average income & $238(49.8)$ & $116(49.6)$ & $122(50.0)$ & & & \\
\hline Diabetes medication type, n (\%) & & & & 3.6 & & .17 \\
\hline OHA(s) only & $294(61.5)$ & $143(61.1)$ & $151(61.9)$ & & & \\
\hline Insulin therapy only & $32(6.7)$ & $11(4.7)$ & $21(8.6)$ & & & \\
\hline $\mathrm{OHA}(\mathrm{s})$ and insulin therapy & $152(31.8)$ & $80(34.2)$ & $72(29.5)$ & & & \\
\hline Recruitment nurse, n (\%) & & & & 0.2 & & .62 \\
\hline Practice nurse & $342(71.5)$ & $165(70.5)$ & $177(72.5)$ & & & \\
\hline Diabetes nurse & $136(28.5)$ & $69(29.5)$ & $67(27.5)$ & & & \\
\hline Depression status, n (\%) & & & & 1.2 & & .27 \\
\hline Never/in the past & $452(94.6)$ & $224(95.7)$ & $228(92.4)$ & & & \\
\hline Current $^{\mathrm{a}}$ & $26(5.4)$ & $10(4.3)$ & $16(6.6)$ & & & \\
\hline $\mathrm{HbA}_{1 c}(\mathrm{mmol} / \mathrm{mol})$, mean $(\mathrm{SD})^{\dagger}$ & $56.9(11.8)$ & $56.6(11.8)$ & $57.1(11.8)$ & & -0.42 & .67 \\
\hline BMI, mean (SD) & $31.0(5.0)$ & $30.8(4.9)$ & $31.2(5.1)$ & & -1.01 & .31 \\
\hline OHA Adherence score, mean (SD) & $13.2(3.8)$ & $13.5(3.5)$ & $12.9(4.0)$ & & 1.45 & .15 \\
\hline IT Adherence score, mean (SD) & $7.7(1.7)$ & $7.4(1.8)$ & $7.9(1.6)$ & & -1.87 & .06 \\
\hline Physical activity (min), mean (SD) ${ }^{\ddagger}$ & 815 (919) & $868(1031)$ & $764(796)$ & & 1.24 & .22 \\
\hline Snack intake (cal), mean (SD) ${ }^{\ddagger}$ & $1710(1404)$ & $1746(1435)$ & $1676(1375)$ & & 0.55 & .58 \\
\hline
\end{tabular}

$\mathrm{SD}=$ standard deviation, $\mathrm{OHA}=$ oral hypoglycemic agent(s), IT = insulin therapy, $\mathrm{HbA}_{1 c}=$ glycosylated hemoglobin, $\mathrm{BMI}=$ body mass index (weight/length²)

${ }^{+}$This equals an $\mathrm{HbA}_{1 \mathrm{c}}$ of $7.4 \%$

* Weekly mean minutes/calories 
Table 2 Results of linear mixed regression analysis: full model and model after backward elimination

\begin{tabular}{|c|c|c|c|c|}
\hline Full model & $\begin{array}{l}\text { Regression } \\
\text { coefficient }\end{array}$ & $\begin{array}{l}\text { 95\% Confidence } \\
\text { Interval }\end{array}$ & T Value & $P$ value \\
\hline \multicolumn{5}{|l|}{ Trial arm } \\
\hline $\begin{array}{l}\text { Intervention arm } \\
\text { Control arm }\end{array}$ & .377 & .099 to .655 & 2.68 & .01 \\
\hline $\mathrm{BMI}$ & -.004 & -.031 to .022 & -.34 & .74 \\
\hline \multicolumn{5}{|l|}{ Recruitment nurse } \\
\hline $\begin{array}{l}\text { Practice nurse } \\
\text { Diabetes nurse }\end{array}$ & -.123 & -.475 to .228 & Diabetes nurse ${ }^{a}$ & .49 \\
\hline Diabetes medication type & & & & .44 \\
\hline OHA(s) only & .163 & -.162 to .488 & .99 & .32 \\
\hline $\begin{array}{l}\text { Insulin therapy only } \\
\mathrm{OHA}(\mathrm{s}) \text { and insulin therap }\end{array}$ & \multicolumn{3}{|c|}{$\mathrm{OHA}(\mathrm{s})$ and insulin therapy ${ }^{\mathrm{a}}$} & .57 \\
\hline $\begin{array}{l}\mathrm{HbA}_{1 \mathrm{c}} \\
\text { Depression status }\end{array}$ & \multicolumn{3}{|c|}{ Depression status } & .42 \\
\hline \multicolumn{4}{|l|}{ Current $^{a}$} & .49 \\
\hline \multicolumn{5}{|l|}{ Gender } \\
\hline \multicolumn{5}{|l|}{ Female $^{a}$} \\
\hline Age & -.019 & -.047 to .009 & -1.32 & .19 \\
\hline Work status & & & & .20 \\
\hline Salaried/Self employed & -.403 & -.893 to .086 & -1.63 & .11 \\
\hline No salaried employment & -.587 & -1.139 to -.035 & -2.10 & .04 \\
\hline $\begin{array}{l}\text { Retired } \\
\text { Disabled/Incapacitated }\end{array}$ & \multicolumn{3}{|c|}{ Disabled/Incapacitated ${ }^{\text {a }}$} & .33 \\
\hline \multicolumn{5}{|l|}{ Living arrangement } \\
\hline $\begin{array}{l}\text { Together with partner } \\
\text { Alone }^{\mathrm{a}}\end{array}$ & .258 & -.063 to .580 & 1.59 & .11 \\
\hline Education level & & & & .23 \\
\hline Low & -.268 & -.593 to .057 & -1.63 & .11 \\
\hline Middle & -.039 & -.393 to .316 & -.22 & .83 \\
\hline \multicolumn{5}{|l|}{ High $^{a}$} \\
\hline \multicolumn{5}{|l|}{ Net. Income } \\
\hline $\begin{array}{l}\text { Under average income } \\
\text { Above average income }\end{array}$ & .300 & -.035 to .635 & 1.77 & .08 \\
\hline $\begin{array}{l}\text { Model after backward } \\
\text { elimination }\end{array}$ & $\begin{array}{l}\text { Regression } \\
\text { coefficient }\end{array}$ & $\begin{array}{l}\text { 95\% Confidence } \\
\text { Interval }\end{array}$ & T Value & $P$ value \\
\hline \multicolumn{5}{|l|}{ Trial arm } \\
\hline $\begin{array}{l}\text { Intervention arm } \\
\text { Control arm }{ }^{\mathrm{a}}\end{array}$ & .243 & .005 to .481 & 2.006 & 0.046 \\
\hline
\end{tabular}

a Reference category 


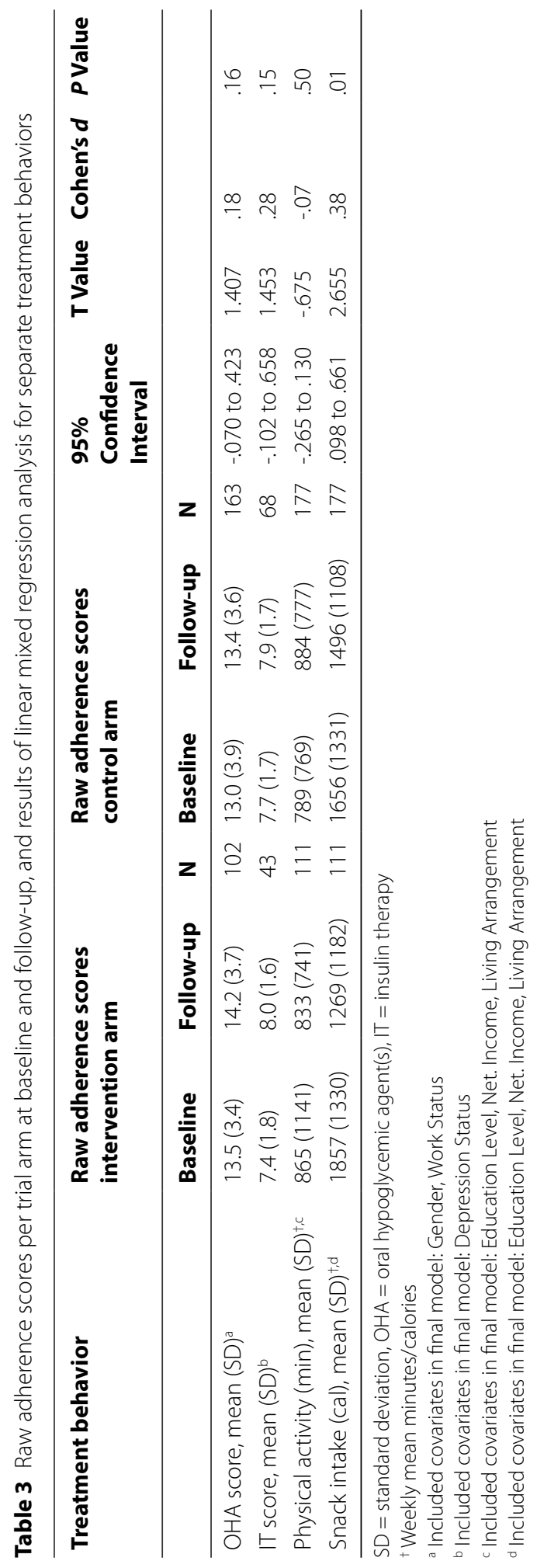




\section{Discussion}

This study examined the effectiveness of a novel web-based computer-tailored program, 'My Diabetes Profile, on overall adherence to core treatment behaviors in patients with T2DM. Additionally, we explored the effects of the MDP-program on each separate behavior. The MDP-program improved overall adherence with a small to medium significant effect size ( $d$ $=.24$ ) and in particular a positive effect was seen in decreasing caloric intake from unhealthy snacks.

Our study is one of the few to focus on improving adherence to multiple treatment behaviors in T2DM, and is to our knowledge the first to subsequently quantify program effects in terms of an effect size. Generally, there is in intervention studies limited emphasis on exploring overall change across risk behaviors (193). Most studies focused on improving separate treatment behaviors, and the few targeting multiple risk behaviors have mainly examined change in separate risk behaviors $(70,71)$. When risk behaviors co-occur, which is the case in the vast majority of patients with T2DM, the adverse effects on health and health outcomes are the largest $(25,165,193)$. Evaluating the effect of multi-behavior interventions requires methods to quantify changes across several behaviors. Prochaska and colleagues (165) supported to quantify the overall change in multiple behaviors by first calculating standardized change scores of each separate behavior and subsequently adding these scores, see e.g. $(193,197)$. However, although the application of such a composite change score has considerable advantages, it may be difficult to interpret as it is an abstract number. After transformation to a standardized composite change score, treatment effects resulting from the analysis are effect sizes (Cohen's $d$ ) and we believe that such a transformation increases the interpretability of observed results. To our knowledge, no studies targeting improvements in adherence to T2DM treatment behaviors through eHealth have quantified an overall intervention effect in terms of an effect size. Such an effect size allows for comparison between distinct multibehavior interventions, and examines overall impact on health behavior change, which is not possible when focusing on change in separate behaviors (193, 198). We recommend future studies to report multi-behavior intervention effects in terms of effect sizes in order to improve interpretability and allow comparisons across studies.

Findings from the current study appear to be robust in the complete case analysis and credible in the sensitivity analyses under the optimistic imputation scenarios. Both complete case and optimistic missing data imputation analyses showed our intervention effect was significantly larger compared to the control arm. However, in the pessimistic missing data imputation scenarios the main effect of the intervention became insignificant. Probably, the complete case scenario is the most accurate reflection of the actual intervention effect in this case, the more as the attrition analysis revealed no presence of differential attrition regarding patient demographics (199-201). 
When inspecting the nature of the overall effect and looking at the individual behaviors, we observed small to medium effect sizes for caloric intake from unhealthy snacks ( $d=.38$ ), insulin therapy adherence $(d=.28)$, and OHA adherence ( $d=.18)$, and a negligible effect size for PA ( $d$ $=-.07$ ). Of these effects, only the effect for caloric intake was statistically significant. Our results are in line with an earlier RCT in patients with T2DM on the effects of a web-based diabetes support program (37). Directly after a four-month intervention, significant improvements in healthy eating $(d=.32)$, fat intake $(d=.28)$, and PA $(d=.19)$ were observed in this trial, but a negligible effect was observed for medication taking (79). The positive effects in both our study and the aforementioned study may be related to specific success factors of (eHealth) interventions. Both interventions incorporated a theoretical foundation, interactive tailored content, and addressed multiple-behaviors involved in the treatment of T2DM. Although it is very difficult to examine the exact effect of such success factors, our findings support the conclusion that interventions applying sound theoretical motivational theories as a basis, interactive tailored content, and a multiple-behavior approach, can have relevant effects (65, $70,71,79,80)$.

The largest effect of our program was seen on eating behavior, i.e. a decrease in caloric intake from unhealthy snacks. Improving dietary patterns in patients with T2DM is multifaceted, and generally a decrease in fat intake is strived for $(77,202)$. Unhealthy snack intake recently emerged as a major novel issue in T2DM patients' diets, according to both health professionals and patients themselves (28) and about a third of our participants selected this behavior to improve in the MDP-program. Perhaps, the novelty of this diet topic and the detailed health risk appraisal complemented with personal feedback may have informed and alerted patients in such a way, that they were triggered to successfully pursue a decrease in their caloric intake via snacks (203). However, more research is needed to examine why the largest effect of our program occurred in caloric intake from unhealthy snacks.

No significant effects were observed for $\mathrm{OHA}$ and insulin therapy adherence, as well as for PA. Observing no effects on PA-levels seems to be common in digital multi-behavior intervention studies $(52,78,154,197,204,205)$. Yet, PA was the behavior most often chosen by patients and they were provided with a detailed health-risk appraisal on their current PA-levels. A more detailed analysis revealed that these patients had a high willingness to increase their PA and almost all could have chosen other topics to improve, so they were not 'forced' to pursue PA improvements. There are several potential explanations for the lack of any effect on PA of our program. Patients could have overestimated their need and willingness to improve PA-levels, as on average in this group guideline targets were six fold higher than the target of 150 minutes PA per week (206). Please note that these were self-reported values and were probably an overestimation, as several studies have shown that many persons are unable to reliably estimate their PA-levels (207). Using modern technology, like accelerometers, this barrier might be overcome in the future (206). It may also be that patients chose PA, as opposed to medication adherence improvements, as patients considered healthy lifestyle domains as 
more crucial to their health than medication taking $(116,208)$. While improvements emerged in the other domains, i.e. unhealthy snack intake, it might have been that improvements in this behavior required considerable self-control efforts. In turn, self-control spent on decreasing caloric intake may have depleted resources for further volitional efforts of for instance improving or maintaining already high PA-levels (209). In fact, recent studies indeed show that high levels of self-control are required to translate short-term intentions into pursued PA improvements (210). However, further research is needed to determine why multi-behavior Internet interventions seem to have such a limited effect on PA-levels. For instance, reasons for failure could be explored in-depth using qualitative interviews, to analyze whether specific and effective action plans were made, and whether PA plans were combined with other adherence activities which could have led to over depletion in certain patients $(209,211)$.

\section{Strengths and limitations}

Primarily, the multi-behavior approach in order to improve treatment adherence is a strength of our study. Existing interventions have largely focused on improving single behaviors, while the management of T2DM is multifaceted and treatment non-adherence co-occurs across treatment behaviors. Second, the program was theory-based and applied previously identified success factors for effective online self-management programs. Third, our nationwide recruitment and quite robust findings enhance the generalizability and credibility of our results. Fourth, our study improved interpretability of the analysis results on the mean difference between intervention and control, by transforming it to an effect size, which also may simplify comparisons of results of similar interventions in meta-analyses (195). Moreover, changes on all behaviors were incorporated in the primary outcome, including changes to those behaviors patients did not choose to receive feedback on in the intervention. Although this could potentially have reduced the effect identified, the overall score allowed us to correct our findings for changes in other behaviors (i.e. improvements or compensation trade-offs) $(116,212)$. Last, we observed a relatively low attrition rate of 40\%; attrition rates reaching $60-80 \%$ are common in web-based interventions (213-215). Limitations of our study are mainly methodological in nature. Firstly, adherence data was collected through self-report questionnaires, which can be prone to social-desirability issues and behavior overestimation $(207,216)$. However, the impact of such biases were likely reduced by applying baseline-follow up change scores and identical adherence assessments for participants in both trial arms. Secondly, we did not assess clinical outcomes such as glycemic control. However, as we did not include a post-intervention follow-up period, it may be unlikely that such outcomes will be observed immediately after our intervention. Finally, we did not assess long-term effects of our MDP-program.

\section{Conclusion and implications}

The MDP-program yielded larger improvements in overall treatment adherence postintervention, compared to our control arm, reflected by a small to medium overall effect size. Changes in separate behaviors yielded a significant small to medium effect size for decreasing 
caloric intake from unhealthy snacks, while small but statistically insignificant effects were observed for medication taking behaviors. Small to medium effect sizes as observed in our study may be of importance when multiplied to the population level (79). Therefore, a widescale program implementation is suggested. Beforehand, some suggestions are at hand in order to increase the likelihood of successful wide-scale implementation delivery. Patients were now recruited by their nurse through consultations, but it is unknown if this delivery strategy is feasible in practice. Potentially, these professionals could benefit from a feedback loop in the program where they can track the progress of their patients regarding treatment adherence, in order to use consultation time dedicated to the program most efficiently. More research is needed regarding the most feasible delivery system for the intervention. For instance, research could investigate health professionals' willingness to adopt and implement the MDP-program, and whether such a feedback loop is wanted. Enhanced follow-up periods following the intervention could assess the sustainability of results observed on short-term, and data could be gathered on secondary outcomes in the personal, economic and societal area. Last, more research is needed to investigate how PA-levels could be improved or maintained through eHealth interventions that aim for adherence improvements in multiple T2DM behaviors. 


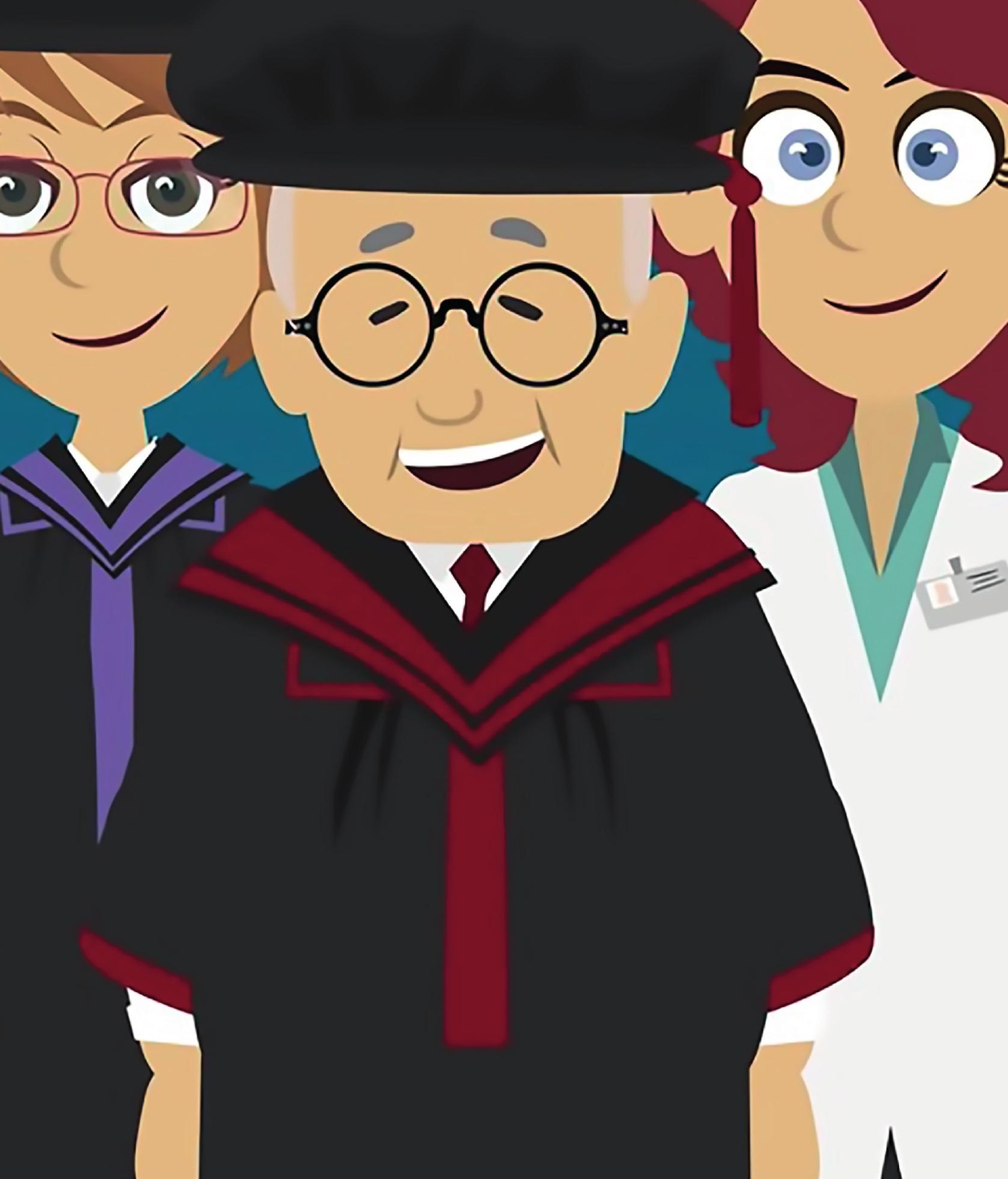


The main aim of the studies displayed in this dissertation was to describe the development and the effectiveness of the eHealth program, My Diabetes Profile, which aimed to improve adherence of patients with T2DM to core treatment behaviors, i.e. improving physical activity levels, oral hypoglycemic agent adherence, insulin therapy adherence, and decreasing caloric intake from unhealthy snacks.

The first part of this dissertation included three chapters; two chapters on two studies conducted as part of the formative research process, and one chapter with a description of the development and content of the My Diabetes Profile program, as well as a blueprint of the randomized controlled trial that was conducted to examine the program's effectiveness. The second part of this dissertation consisted of two chapters related to the evaluation of the My Diabetes Profile program. The first chapter described a study that examined factors influencing recruitment and its success of nurses who engaged in recruiting patients for our trial. The second chapter included a study that assessed the effectiveness of the My Diabetes Profile program on overall treatment behavior adherence and separate treatment behavior adherence in patients with T2DM.

This final chapter summarizes the main study findings, outlines strengths and limitations of the studies described in this dissertation, and discusses research and practical implications. This general discussion ends with a general conclusion.

\section{Formative research, program development, and trial protocol}

\section{Main findings}

The studies included in the first part in this dissertation were described in chapters 2-4. First, a brief summary of the results is provided of each study, and subsequently the results are discussed by considering study strengths and limitations, and discussing implications for research and practice.

Chapter 2: Exploring beliefs on diabetes treatment adherence among patients and health professionals.

We explored the scope of patient (non)-adherence and associated beliefs and determinants among both patients themselves as well as health professionals directly involved in their T2DM management. Our results showed that treatment adherence to core T2DM strategies was suboptimal. Non-adherence was mainly reported in dietary patterns and medication taking behaviors. Patients indicated to adhere to physical activity (PA) guidelines, while health professionals held the opposite perspective. Unhealthy snack intake was reported by both groups as important contributor to unhealthy diets of patients with T2DM. 
Patients' knowledge and risk perceptions regarding PA were considered sufficient by both health professionals and patients. With regard to healthy diets, health professionals indicated that these factors were insufficient, contrary to perceptions of patients themselves. Both groups reported that knowledge and risk perceptions related to medication taking could be improved. Health professionals and patients reported many advantages and no disadvantages of adherence to PA and healthy diets. Both advantages, e.g. feeling healthier, and disadvantages, e.g. a variety of potential adverse side effects were mentioned for medication taking. Patients' self-efficacy to adhere to treatment behaviors seemed to be lower in out of daily routine situations, such as snacking when being on vacation. Across treatment behaviors, similar situations occurred in which self-efficacy to adhere to the particular behavior was lower.

It seems that success factors for treatment adherence were insufficiently applied in consultations, i.e. patient preparation, collaboration between a health professional and patient in formulating treatment strategies, (clear) goal-setting, and self-management education, i.e. the process of informing, motivation, and equipping patients to manage T2DM and support treatment adherence outside the clinical setting. Health professionals reported that scarce, busy, and brief consultations may impede the application of such success factors.

Chapter 3: Psychological predictors of adherence to oral hypoglycemic agents: An application of the ProMAS questionnaire.

A longitudinal study design was applied with surveys at baseline and six-months followup. At baseline, oral hypoglycemic agent $(\mathrm{OHA})$ adherence using the ProMAS and MARS-5, socio-cognitive determinants, and demographics were assessed. At follow-up, the ProMAS was applied as outcome measure, on which socio-cognitive determinants and demographics were regressed using linear regression analysis. When applying the currently advised and widely applied cut-off points of $\geq 15$ for the ProMAS and $\geq 23$ for the MARS-5, $47.7 \%$ and $89.5 \%$ adhered to their oral agents respectively. Data of the ProMAS were more in line with objectively collected data with regard to the percentage of patients that could be considered adherent. Consistent predictors of better adherence were a low education, lower severity perceptions, and higher self-efficacy and intention. After correcting for baseline adherence, a low education and higher self-efficacy remained significant predictors. The final model explained $48 \%$ of the variance in adherence behavior.

Chapter 4: A web-based program to improve treatment adherence in patients with type 2 diabetes: Development and study protocol.

Findings from chapters two and three were complemented with knowledge accumulated from previously developed computer-tailored programs and input gathered from a program committee. Together these sources yielded input for the development of the eHealth program My Diabetes Profile. Consequently, the program addressed treatment non-adherence to 
core T2DM treatment behaviors, and aligned with the needs of our target group and those involved in the committee. In addition, the program incorporated success factors for treatment adherence as identified from the literature, i.e. a solid theoretical base, interactive content and feedback tailored to relevant phases and determinants of behavior change, visual support, and the possibility to monitor adherence.

\section{Discussion of findings and research implications Treatment behavior adherence}

Findings from the formative studies contributed to the understanding that treatment adherence was suboptimal and that non-adherence co-occurred across behaviors. Our results support the findings already described in earlier literature available on patient adherence to T2DM treatment behaviors: dietary and PA guideline targets are not met adequately, and many studies on adherence to pharmacological strategies report adherence prevalence percentages below 80\%, which is generally considered suboptimal adherence $(23-25,29$, 30). The successful management of diabetes is complex and burdensome process for many patients as it frequently should include several healthy lifestyle interventions in combination with the use of multiple drugs - sometimes prescribed several times a day - and each of these medications can have side-effects. Below 5\% of the patients with T2DM adheres to all treatment behaviors, while over $80 \%$ could improve at least two behaviors (25). Inadequate adherence could result in avoidable suffering of the patient, and is associated with considerable adverse personal, societal, and economic consequences (7, 17-22, 217). Moreover, if risk behaviors occur simultaneously, adverse effects on health and health outcomes may even be greater, while improving multiple behaviors may potentially result in greater health benefit (198). Therefore, it is recommended to apply a multi-behavior approach, covering both healthy lifestyle and medical behaviors, in attempts to improve adherence and ultimately glycemic control.

A discrepancy seems present in patients' awareness with regard to their diet. Although patients in our study indicated to maintain a healthy diet, an in-depth review of their eating pattern revealed that unhealthy snack intake was highly prevalent in their diet and accounted for a substantial amount of caloric intake; this issue was also identified by health professionals. The intake of unhealthy snacks is an understudied issue in the dietary pattern of patients with T2DM. Largely, attention has been paid to macronutrients such as fat and carbohydrate intake and their relation to the prevention and management of diabetes (218). A transition towards an increased caloric intake such as from high-energy snacks is observed in many countries and this may have contributed to a current shift of focus from (isolated) macronutrients to food groups (218-220). As a decreased caloric intake is frequently part of the dietary management in overweight patients with T2DM, targeting unhealthy snack intake could contribute to a lower daily energy intake and might also improve glycemic control (1). 
A discrepancy also exists regarding perceptions of health professionals and patients concerning patients' PA adherence. While professionals reported that patients' adherence to PA targets was poor, many patients indicated to be physically active, and mentioned a variety of activities accordingly. This discrepancy may lead health professionals to address the issue of non-adherence to PA targets, while patients may not perceive the necessity of improvements as they already are or assume to be adherent. Research points out that patients tend to overestimate their PA-levels and therefore may not be motivated to improve their PA or perceive PA promoting messages as not relevant (27). For example, adults at high risk for T2DM who either correctly or incorrectly estimated their PA-levels as sufficient, may not perceive improvements in PA-levels as a means to diminish their risk of T2DM (221). Another argument why patients may overestimate their PA-levels relates to the Dutch PA-guidelines. In our study we asked the participants if they perceived to adhere to the Dutch norm of healthy PA-levels of 150 minutes of weekly PA with a Metabolic Equivalent of Task value (MET) of $\geq 4$ $(222,223)$. A MET-value is a physiological measuring unit to quantify the energy expenditure needed for an activity, compared to the energy required in resting metabolism (190). Our patients reported considerable physical activities with a MET-value between three and four METs, e.g. normal speed walking (28). It may have been that participants included these activities in their total, resulting in an overestimation of their PA-levels.

In sum, it seems that the discrepancy with regard to perceptions of health professionals and patients concerning patients' adherence to PA-levels, may be explained by patients overestimating their actual PA-levels. Recently, however, Dutch PA guideline targets have been adapted to be more compatible and better connected to international guidelines (191). Main changes are that in the new guidelines the targeted 150 minutes of weekly PA requires not to be spread out over at least five days (PA divided over several days is still recommended), and that activities carried out less than 10 minutes consecutively are included in the total. In addition, activities with a MET-value of $\geq 3$ may now be included in the total, compared to activities with a MET-value of $\geq 4$ in prior guidelines $(206,222,223)$. Taking into account the alteration with regard to MET-values in new guidelines compared to prior one's, it may be that patients in fact adhere given they reported considerable engagement in three to four MET activities. Still patients could be stimulated to increase their PA, even if targets are already met as guidelines show that more PA leads to more health gain (206). The most viable target for intervention seems to be to increase patients' awareness of either their overestimation of PAlevels, or of the necessity of improvements despite already meeting targets.

Results of both formative studies indicated that patients' adherence to pharmacological strategies was suboptimal, and should therefore be targeted in interventions that aim to contribute to a more favorable management of T2DM. Perspectives of health professionals indicated that patients' medication adherence was poor, and only half of the patients themselves reported to adhere to their diabetes medicines. A similar result was observed when adherence was quantified through application of the novel self-report ProMAS 
questionnaire; $48 \%$ of the patients was considered adherent to their oral hypoglycemic agents. However, when applying the MARS-5, a patient adherence prevalence of 90\% was observed. Divergent suboptimal results regarding the prevalence of adherence to diabetes medicines have also been reported across the literature, ranging from 39-93\%, and are attributable to a wide variety of adherence measures applied (30). Existing self-report measures tend to yield skewed adherence distributions, and in general the adherence prevalence is estimated to be around 50\% (124). Data collected through the novel ProMAS, which was developed to deal with flaws in existing instruments, show a similar percentage as to objectively collected data in terms of adherence percentages (125). Preferably, performance comparisons between selfreport measures to assess adherence such as the ProMAS are complemented with a more objective instrument, such as Medication Possession Ratio's or Medication Event Monitoring Systems $(122,129)$. In order to obtain results from the exact same study population contrary to comparing results across studies and study populations, future research could simultaneously apply the ProMAS and a more objective measure, as a combination is considered to provide the most accurate estimation of adherence (129).

In order to assess insulin therapy adherence, we applied an adapted version of the ProMAS questionnaire, including nine of the original eighteen items (124). Some items that are relevant to assess oral medication adherence, may be irrelevant regarding insulin therapy adherence. For instance, the item 'I sometimes take fewer medicines than prescribed by my doctor', does not distinguish between adherence and non-adherence for insulin therapy. Therefore, this item was removed from the original questionnaire as patients using insulin should adjust their dosage in case of hypoglycemia or illness. In line with cut-off points for the original ProMAS, we adapted the nine-item questionnaire for insulin therapy adherence accordingly. A systematic review on techniques to assess insulin therapy adherence in patients with T2DM, including self-report measures, concluded that these instruments entailed considerable measuring challenges, complex forms of data collection, and had no cut-off points for adherence or reliable associates of adherence outcomes. None of the identified instruments were appropriate for use as a quality measure for insulin therapy adherence (224). The original ProMAS showed to be a promising questionnaire to assess adherence to oral hypoglycemic agents as its data were less skewed towards adherence, similar to objectively collected data, and yielded insights in a broader spectrum of (non)-adherence behaviors, compared to the frequently applied MARS-5 (125). Therefore, we believe that the adapted ProMAS for insulin therapy may be as promising as the one applied for OHA adherence. However, future research should further assess its validity and applicability.

\section{Determinants of treatment adherence}

Perceptions of patients and health professionals sometimes seemed to differ regarding whether patients had sufficient knowledge, were aware of risks of non-adherence, and were correctly aware of their own behavior. The I-Change Model postulates that knowledge, risk perceptions, and the level of cognizance are important precursors of motivation (43). A person's 
cognizance level indicates if a person is (in)correctly aware of carrying out the recommended behavior. A discrepancy in perceptions could potentially lead health professionals to motivate patients to change their adherence behavior, while in fact they may not be aware of the necessity to change. Contrary, if patients indeed show sufficient awareness, the model posits that health professionals should rather intervene in a more proximate behavior change phase to provide patients with relevant feedback accordingly. It is known that both nurses and patients may lack knowledge on diabetes care, e.g. nurses on diet and physical activity, and patients on their insight in one's own behavior $(63,225)$. To overcome discrepancies in perceptions of health professionals and patients, an implication could be to primarily assess the patient's knowledge, risk perceptions, and cognizance levels. Subsequently, if it turns out that insufficient awareness of the necessity of behavior change is present, it is evident to tailor content to this behavior change phase to provide patients with relevant information. Contrary, if sufficient awareness is shown by the patient, the behavior change process could proceed to the motivational phase (43).

Patients' self-efficacy to adhere to distinct treatment behaviors seemed to be lower in certain out of routine situations. If daily routine is retained, individuals acquire self-knowledge by repeating a specific action; at a certain point no judgement of efficacy is required on such habitual occasions (226). Adherence across behaviors may therefore be more prone to failure when routine is compromised. A recent review also showed that self-efficacy beliefs were decreased in different risk situations across a wide variety of health behaviors, e.g. condom use in risky sexual practices (227). Therefore, it seems evident to increase patients' self-efficacy in these out-of-routine occasions to enhance the chance of successful adherence despite being faced with a difficult situation. Potentially, an increase in self-efficacy could be facilitated by coping planning. For instance, individuals could first be prompted to identify their personally relevant risk situations in which adherence may be prone to failure, and anticipate these by forming a coping response on how to overcome failure if the situation would occur $(43,47)$. This way, the likelihood of behavior success is increased despite being faced with difficulty.

Although we explored beliefs within the pre-motivation and motivation phase in changing behaviors, we did not manage to assess those for the post-motivation phase. It is not unlikely that patients who express a high willingness to pursue adherence, will fail to achieve their intentions, as this requires considerable self-regulatory capacities (56). We suggest that future studies should explore salient beliefs related to the post-motivation phase, e.g. on how to anticipate low self-efficacious situations, on what goals are feasible and realistic, and on how to prepare an intended behavior. Such factors - also referred to as action plans - are likely to facilitate enactment of intentions, and could yield information to be addressed in interventions (56), and have been shown to increase the chance of translating intentions into the desired goal behavior $(46,49,50)$. Consequently, these factors were addressed in our intervention. 


\section{Success factors for treatment adherence}

We observed that success factors for treatment adherence are insufficiently applied in consultation; scarce, busy, and brief consultations may impede the application of such factors. In the Netherlands, consultation sessions between health professionals and patients occur every three to six months and last about 15-20 minutes (63). These consultations are highly protocolled, meaning that many topics such as glycemic control, cardio-vascular riskmanagement, diabetic complications and therapy adherence should be addressed (14). Consultations should ideally serve as a setting where treatment strategies are formed, and where patients are informed, educated, motivated, and equipped with skills to optimally selfmanage and adhere to T2DM treatment strategies in everyday life (105). A collaborative care strategy should be a key part of diabetes consultations according to Dutch care guidelines and its application could result in improved execution of suggested treatment recommendations $(14,115)$. Not only passive patient preparation and involvement are at the basis of insufficient collaboration. Other contributors include scarce time, the difficulty health professionals experience to involve patients in decision making, and the misconceptions between professionals and patients about if treatment goals are actually set. Research posits that health professionals may lack the required knowledge, motivation, and confidence to involve patients in decision making, and to address the issue of non-adherence in consultations $(63,64,228)$. It is advised that professionals should consider the motives and barriers of their patients to perform healthy lifestyles, as they also should with regard to medication taking, and that they tailor advice to the needs of their patients. The complexity of the behavior change process requires nurses to shift to a counseling-based approach. Such an approach of informing and motivating patients to improve on multiple behaviors, in a supportive, comprehensive and barrier-focused manner is a challenge in daily practice as a multitude of other factors need to be addressed $(63,229)$. Although it seemed that the lack of application of success factors for treatment adherence occurred at both the patient and health professional side, a main common barrier seemed to be the time constraints of scarce and relative short consultations; a common barrier to the conduct of healthy lifestyle counselling. Professionals do indicate the importance of addressing these factors and promoting healthy lifestyles, however, they simply do not have enough time to address them in consultations $(62,229,230)$. To conclude, it seems that consultation sessions between health professionals and patients are not an ideal setting to thoroughly discuss the issue of non-adherence, to make efforts to assist in the ongoing and complex process of health behavior change, and to address success factors for treatment adherence. Avenues need to be explored that could alleviate pressure on the healthcare system, and still deliver patients the required ongoing support they need in order to optimize adherence to T2DM treatment strategies.

\section{Practical implications}

The highly protocolled diabetes consultations in the Netherlands provide guidance and structure to those topics that need to be discussed (14). However, it seems that success factors for treatment adherence are insufficiently applied, and that the nature of consultations does 
not allow for thoroughly discussing the issue of non-adherence or to make efforts to assist in the ongoing and complex process of health behavior change. A practical consideration could be to outsource the complex area of behavior change to a more feasible context, for example the context of Internet. The Internet has widely and successfully been applied for health services and health promotion activities such as the management of T2DM, and such activities delivered through the Internet are often referred to as eHealth $(65,69-71)$. An eHealth program could assess and identify risk behaviors, and assess relevant determinants and beliefs to intervene in for those behaviors subject to improvement, in order to aim for improvements. Given our findings from formative research studies, such an eHealth program could focus on assessing adherence to healthy lifestyles, i.e. PA-levels and unhealthy snack intake, and adherence to pharmacological strategies, i.e. adherence to OHAs and/or insulin therapy. An assessment of determinants and beliefs may yield information on relevant intervention targets, following the behavior change phases as suggested in integrative models such as the I-Change Model (43).

Besides focusing on improvements in treatment non-adherence, an eHealth program could address treatment adherence success factors that are not sufficiently applied in consultations, such as patient preparation and active engagement. There may be no need to fully abandon discussing treatment adherence and the application of success factors in consultations between professionals and patients. A recent review on person-centered eHealth in patients with chronic illnesses, posits that eHealth integrated into usual care can help patients to manage their chronic illness better, and will provide better quality of care in long-term (231). An integration of eHealth with care as usual, referred to as 'blended care', provides opportunities to transfer information, and to facilitate communication between health professionals and their patients (231). A combination of face-to-face counseling with ongoing online support is suggested as a means to approach the etiological problem of diabetes. Thereby, a personalized approach to target lifestyle non-adherence may not only facilitate the management of T2DM but also contribute to the management of the disease and economic gain (232). To facilitate discussing treatment adherence in consultations efficiently in light of scarce, busy, and brief sessions, the program could transfer relevant information on patient activity in the program to their own nurse, for example, the goals a patient has set, and monitoring the treatment behavior a patient is currently working on. In addition, the program could prompt patients to consider topics or questions to discuss with their health professional as health professionals indicated to welcome a more prepared and active patient (233). 


\section{Recruitment and effectiveness evaluation}

\section{Main findings}

The studies included in the second part in this dissertation, were described in chapters 5 and 6. First, a brief summary of the results is provided of each study, and subsequently the results are discussed by considering study strengths and limitations, and discussing implications for research and practice.

Chapter 5: Exploring factors influencing recruitment success of nurses recruiting diabetes patients for a randomized controlled trial.

Ninety-six nurses participated in our trial and recruited on average seven patients (range 0-32). Fifteen nurses did not recruit any patient. Most patients were recruited close to recruitment onset. Nurses who did not recruit patient close to recruitment onset, ended up recruiting no patients. Data show a relative high early recruitment success that progressively declined over time; high-recruiters were generally successful recruiters throughout the entire recruitment period. Recruitment barriers and facilitators comprised of organizational, study, patient and especially recruiter characteristics. Contrary to non- and low-recruiters, medium- and high-recruiters reported more in-depth knowledge about the study and trial requirements, expressed more personal benefits of participation and fewer barriers, these nurses applied more recruitment activities, reminders, and barrier-focused coping strategies.

Chapter 6: The effectiveness of a web-based computer-tailored program to improve treatment adherence in patients with type 2 diabetes: A randomized controlled trial.

After the follow-up assessment, 288/478 (60\%) participants were retained from those who initially completed the baseline assessment. After controlling for relevant confounders, overall treatment adherence improved significantly in those patients allocated to the intervention, reflected by an overall significant small to medium effect size $(d=.24)$. With regard to separate treatment behaviors, a significant decrease was observed in caloric intake from unhealthy snacks $(d=.38)$. Small to medium positive intervention effects were observed for adherence to OHA $(d=.18)$ and insulin therapy $(d=.28)$, however, these effects were not statistically significant, probably due to a lack of power in these behaviors.

Success factors for eHealth interventions identified across the literature and applied in our intervention, e.g. a theoretical base, tailored content and a multi-behavior approach, may have contributed to the success of the intervention. 


\section{Discussion of findings and research implications Evaluation of nurses' recruitment success}

In trials, patient recruitment often occurs through efforts of health professionals, given their direct and relevant contact to the patient $(89,90)$. Therefore, in our trial, practice nurses and diabetes nurses were invited to aid in recruiting eight patients with T2DM to trial within a period of six months. We aimed to initially recruit approximately 700 patients, i.e. 150\% of the required 464 patients with a completed baseline assessment, as not all patients were expected to participate after being recruited by their nurse.

Ninety-six nurses recruited a total of 669 patients to our trial of which 478 completed the baseline assessment. In that light, it can be concluded that our recruitment was successful. Still, these results showed that approximately 30\% of patients did not enact their expressed willingness towards their nurse to participate. To our knowledge, no studies have explored why patients with T2DM omit completing a first assessment, after being positive towards their nurse about participation, and the available literature focusses on drop-out after having entered the trial (234). Upon expressing willingness to participate towards their nurse, patients were registered, given time to rethink participation, and provided with more extended information before the online informed consent procedure would occur. It may be that patients experienced pressure to decline study participation as suggested by their nurse, or that they were in fact pushed by the nurse to commit to participation. Other reasons may be that patients actually forgot to follow-up their agreement, that the extended information withheld them from continuing, that the baseline assessment required too much effort to be completed, or that technological issues disabled them to continue. Cleary, further research is needed to explore and understand why participants who are initially recruited by their health professional to participate in a trial, fail to complete the first stage, i.e. the baseline assessment.

In line with the before mentioned, this study only evaluated initial enrolment of patients by nurses rather their retention in the trial, which is primarily important when considering sufficient study power. Therefore, research could investigate the relation between initial recruitment success, and retention rates throughout the entire follow-up of the trial.

If patient recruitment is regarded as a behaviour, its success seemed to be influenced by several facilitators and barriers identified in the area of organizational, study, patient and especially recruiter characteristics. Concerning recruiter characteristics, a similar pattern could be observed as to exploring treatment behaviour adherence according to the I-Change Model (43). For instance, medium- and high recruiters showed more knowledge about the study and trial requirements, and reported advantageous beliefs and fewer barriers towards recruiting patients, compared to non- and low-recruiters. Moreover, the prior group incorporated more recruitment activities, reminders, and barrier-focused coping strategies. Initially, all nurses expressed their willingness to contribute to recruiting patient to the trial, however, it is wellknown that not all professionals who intend to recruit patients, will actually succeed (89). 
Awareness factors such as knowledge, and motivational factors such as attitudes and selfefficacy are known to be precursors of an intention one has towards performing a behavior (45). It seemed that the intention to recruit patients of non- and low-recruiters was insufficiently grounded, as they showed poor study knowledge, few advantageous beliefs towards recruiting patients, and considerable recruitment barriers. For instance, time constraints and work pressure were often brought up, justifying their recruitment efforts and success; competing demands and time pressure are well known reported barriers to recruitment success, and impede the incorporation of research activities in daily practice $(173,180,182,183)$. Although as with health behaviors a multitude of factors may provide relevant intervention targets to improve successful recruitment, recruiter factors, such as their knowledge of and attitudes towards recruiting patients may be the most viable and changeable domain to intervene in (33). Explorative studies as the current inquiry may not result in conclusive evidence on what determines success, but yielded an in depth exploration of salient beliefs influencing recruitment success. Hence, findings observed should be tested through quantitative study designs, in which for instance also the contribution of organizational, study, and patient characteristics is examined in nurses' recruitment success. As nurses in primary and secondary care participated in our trial, it may also be relevant to explore if beliefs and determinants of recruitment success differ between those nurses, in order to provide either group with relevant tailored advice on how to optimize recruitment efforts and success.

\section{Evaluation of program effectiveness}

The My Diabetes Profile program is to the best of knowledge one of the few interventions to address both healthy lifestyle and medical behavior adherence, and the first to quantify the intervention effect in terms of an overall effect. Since most studies have focused on improving adherence to separate treatment behaviors, e.g. either physical activity or medical behavior adherence, there has been limited evidence on exploring overall change across risk behaviors $(70,71,193)$. Programs that address multiple behaviors require methods to quantify change across those behaviors in order to evaluate their overall effect. In the current study we developed a standardized change score for each separate behavior. Subsequently a composite change score could be calculated for each individual. Such a composite score allows for comparison between distinct multi-behavior interventions, and examines overall impact on health behavior change, which is hampered when only focusing on changes in separate behaviors $(193,198)$. A small number of studies are available that quantified overall change in multiple behaviors through a composite change score as suggested by Prochaska and colleagues (165), however, these study populations differed from our participants. An intervention applying tailored mobile technology coaching in order to improve PA as well as fruit and vegetable consumption, and to decrease sedentary time and fat intake, reported a composite change score of around 80 (197). In a recalculation of two multi-behavior studies, Drake et al (193) demonstrated composite change scores of .82 and 1.05 of two interventions targeting cancer prevention through increases in PA, multivitamin intake, and fruit and vegetable consumption, and a decrease in red meat intake $(235,236)$. The number of targeted 
behaviors in our intervention is equal to those addressed in the before mentioned studies, and a similar composite change score was observed in our study (.85; calculated based on the data reported in chapter 5). However, the different behaviors targeted across different study population, hampers clear comparisons. Moreover, although the application of a composite chance score has considerable advantages, it may be difficult to interpret. A possibility to increase interpretability is to transform, i.e. standardize, the composite change score by dividing the composite change scores for each individual by the pooled standard deviation of these composite change scores. Subsequently, a mean of the resulting 'standardized composite change scores' could be calculated per trial condition. The difference in these means gives information about the direction and the magnitude of the difference between groups, and can be interpreted as Cohen's $d$ (effect size) for the difference between treatment and control on the variable 'change in treatment adherence as a whole' $(193,194)$. Therefore, we recommend future studies to report results in terms of Cohen's $d$ in order to improve interpretability and allow comparisons across studies. As the management of T2DM generally consists of treatment strategies in the area of improving physical activity, dietary patterns, and medication taking, another possibility could be to combine the two medication adherence measures, i.e. merging $\mathrm{OHA}$ and insulin therapy adherence into a combined adherence score for medication taking (as the study power for these behaviors was relatively low). This was beyond the scope of the current dissertation, but potentially worthwhile exploring in future studies.

Factors have been identified in the literature that seem to enhance the likelihood of an Internet interventions aimed at improving treatment behavior adherence being successful. Most eHealth interventions in the area of T2DM management have only to a small extent applied such 'success' factors, i.e. the application a solid theoretical framework, the possibility to identify behaviors at risk, the application of goal-setting and the possibility to self-monitor one's behavior, the incorporation of interactive, tailored, and visual supported content, and aligning content on distinct behavior change phases, i.e. awareness, motivation, and selfregulation $(65,70,71,79,80)$. The positive effect observed in our study and the study of Glasgow et al (79) may be related to the incorporation of success factors in these interventions; both included a theoretical basis, interactive and tailored content, and addressed multiplebehaviors involved in the treatment of T2DM. However, it is difficult to assign the success of these interventions directly to addressing these factors. Although our findings support the assumption that success may be enhanced through their application, more research is needed to assess what factors determine success of eHealth interventions targeting improvements in T2DM treatment adherence.

Our intervention entailed some level of blended care as the My Diabetes Profile program enabled patients to transfer relevant program activity to their nurse, similar to the summary of activity that was transferred to the nurse in the It's Life project (237). In addition to tailored behavioral modules, the program included several general modules, for example 'My Care' 
and 'My Values'. My Care facilitated patients in preparing their consultations and stimulated them to proactively formulate questions, provide a brief health update, and to learn how to be an active collaborator in consultations. My Values enabled patients to enter and visually keep track of their blood glucose levels, blood pressure, weight, and Body Mass Index. Data of these modules, together with relevant information from the tailored behavioral modules - the selected behavior to work on and the set goals by the patient - were automatically summarized and directly made available for the nurse to review. The approach where information can be transferred from the program to a health professional, and where patients can become more self-managed, may provide better care and results in more efficient consultations (231). Unfortunately, data on how exchange occurred between the patient and the nurse were not gathered and is recommended for future research. Similarly, we were not able to evaluate whether consultations were more efficiently organized. Moreover, it is unclear what the best level of integration of eHealth with care as usual will be. These topics should therefore be subject to future research, for example by exploring beliefs among nurses and patients on the blended care approach and its outcomes, and actual exchange of information about goals set and enacted upon.

Finally, two suggestions for future research include the assessment of cost-effectiveness of our eHealth intervention, and the application of longer follow-up periods. As the number of people living with T2DM as well as related costs of care are increasing, it is evident to explore interventions that can deliver relevant care that is not only effective, but also costeffective. Computer-tailored eHealth interventions have shown to be cost-effective, however, it is unclear if the current intervention, for example compared to care as usual, is a profitable alternative $(75,238)$. We therefore suggest to also evaluate the cost-effectiveness of the My Diabetes Profile program. We did not assess clinical outcomes, e.g. glycemic control, which is an important indicator of how well patients' T2DM is controlled. No post-intervention follow-up period was included on top of our six-month intervention trial. It is acknowledged that adherence to both healthy lifestyle behaviors and medical behaviors are important in contributing to glycemic control. As the level of glycosylated hemoglobin $\left(\mathrm{HbA}_{1 c}\right)$ is an estimate of the average blood glucose levels over the past two to three months, it is unlikely to observe effects directly after the intervention. Patients could on average improve on multiple treatment behaviors. As behavior change was aimed for sequentially, it may have been that results on $\mathrm{HbA}_{1 c}$-levels only occur after the program has fully been completed, i.e. at the point that multiple subjects to improvement are addressed. Prospective trials should therefore aim for adequate post-intervention follow-up periods.

\section{Practical implications}

Based on the evaluation studies presented here, some practical implications can be considered. When nurses or other health professionals are asked to recruit patients for a trial, several measures can be taken in order to increase recruitment rates. It seems evident to assess the underlying reasons of nurses who express their willingness to recruit patients. 
For instance, nurses' knowledge about the background and conduct of the study could be assessed, in order to determine if their knowledge first needs to be improved, to process cognitive information on motivational determinants. Likewise, it requires exploration if expressed intentions are sufficiently grounded by motivational beliefs in order to provide nurses with relevant feedback if necessary. If the intention seems to be sufficiently grounded, researchers should warrant that nurses are facilitated to enact their intention. For instance, researchers could monitor the number of patients recruited per nurse, and assess personally relevant barriers of recruitment.

Of the initial 669 patients in our trial, 80\% was recruited by the 74 nurses who were involved from the beginning of the recruitment period, while $20 \%$ by those 22 who became involved later. After the initial start of the study, researchers are thus advised to maintain involving new health professionals, if feasible, as they can still recruit a considerable number of patients. Last, our findings contribute to the cumulative evidence that early success seems to influence overall recruitment outcomes, and that longer time to enroll a first patients is associated with poor success (172). In that light, health professionals should be encouraged to recruit patients as soon as possible. In doing so, a personalized approach may aid recruiters to become and remain successful. For instance, researchers could provide health professionals with information to increase knowledge and motivational beliefs, or facilitate those who do not seem to be successful despite intending to do so with action planning and barrier-focused coping strategies.

\section{General conclusions}

This dissertation described the development and evaluation of a web-based computertailored program to improve adherence to core treatment behaviors in patients with T2DM. Our results showed the effectiveness the My Diabetes Profile on overall treatment adherence, and with regard to separate treatment behaviors on a decrease in caloric intake from unhealthy snacks. The My Diabetes Profile program was supported by thorough formative research which provided insight in the scope of non-adherence, and relevant determinants and beliefs that play a role in treatment behavior adherence. Scarce, busy, and brief consultations between health professionals and patients may not be ideal to discuss non-adherence and the complex process of behavior change adequately. An eHealth program could potentially offer the ongoing tailored support patients need in improving healthy lifestyle and medical treatment behavior adherence. Moreover, eHealth could address those treatment adherence success factors that are insufficiently applied in consultations, such as patient preparation, active involvement, and clear goal setting. To increase the likelihood of being a successful intervention, eHealth interventions should build on solid theoretical foundations, include tailored and visual supported content, and align with relevant phases of behavior change. Nevertheless, discussing treatment adherence as protocolled discussion topic by Dutch 
guidelines should not be abandoned from consultations. An integration of eHealth and care as usual is proposed, however, this requires further research on its evaluation and optimal implementation format. Also the cost-effectiveness of the My Diabetes Profile requires additional research, as well as the long-term effects on treatment behavior adherence and related health outcomes. In order to be of impact, the reach of a successful intervention should be increased. Implementation challenges need to be explored, for example if the delivery format of the My Diabetes Profile program through nurses in consultations is in fact a feasible delivery strategy, and how information gathered through eHealth can be integrated in the multitude of information systems used in Dutch healthcare.

\section{Acknowledgement}

The studies described in this dissertation were supported by the grant'Optimizing patient care for complex type 2 diabetes patients: the CT2D healthy living eCoach. The grant is part of the MUMC+ 2020 strategy. The funding source had no involvement in preparing or conducting the research or research articles. 


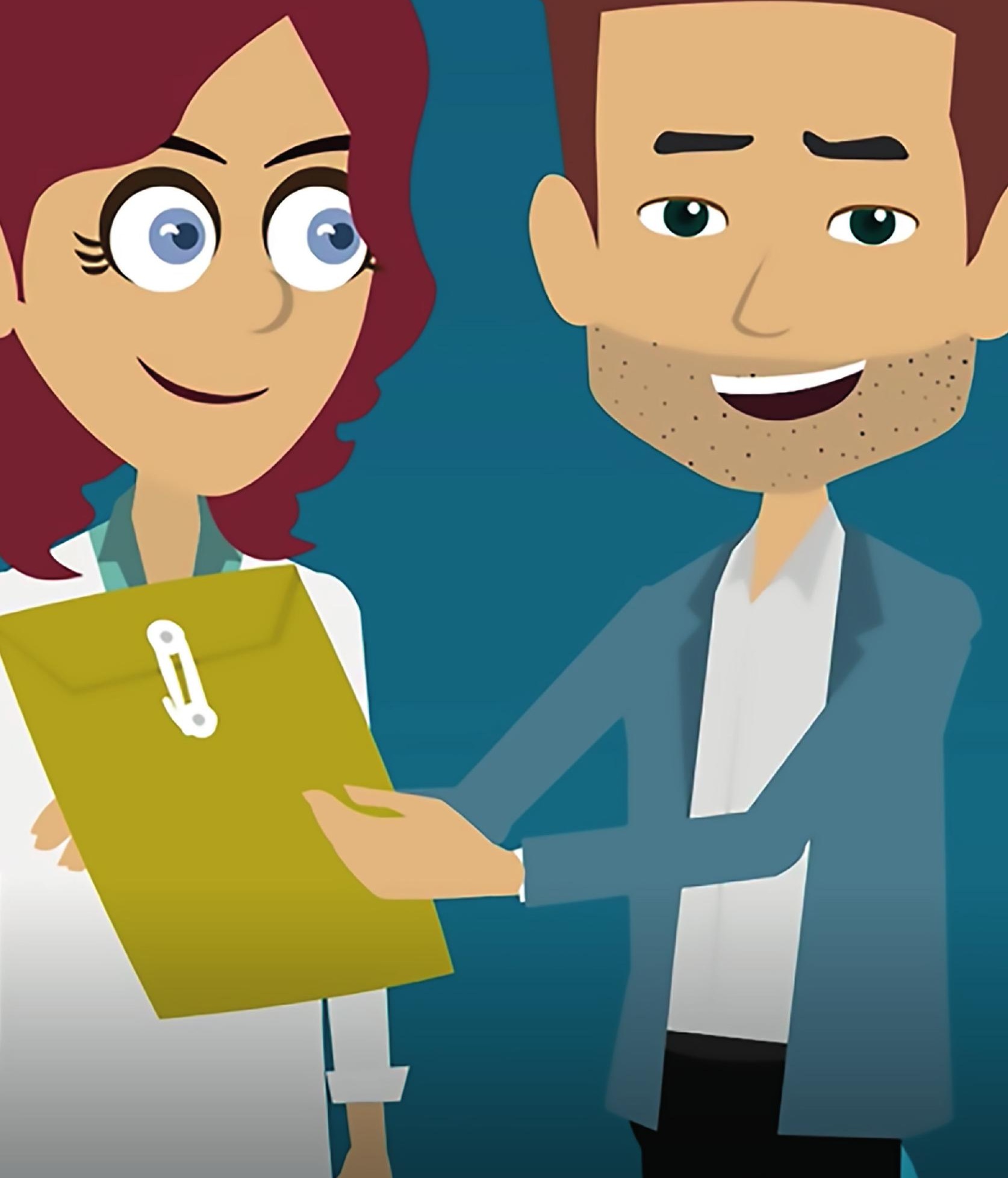


Valorization Addendum 
The main aim of this dissertation was to provide insight in the effectiveness of the newly developed web-based computer-tailoring program 'My Diabetes Profile' that aims to improve treatment adherence of patients with T2DM to core treatment behaviors, i.e. physical activity, healthy dietary patterns and medication taking. Formative research, conducted prior to the development and effectiveness study of the program, was directed at providing insight in the scope of treatment (non)-adherence and its determinants in Dutch patients with T2DM.

The results of the studies presented in this dissertation show that treatment adherence in patients with T2DM is subject to improvement and that patients generally show nonadherence in multiple treatment behaviors simultaneously. As non-adherence is associated with considerable adverse outcomes on personal, societal, and economical levels, it is highly relevant to pursue improvements in patients' adherence. In chapter 6 we demonstrated that the newly developed eHealth program, My Diabetes Profile, has the potential to be an effective intervention to improve patients' overall adherence to T2DM treatment recommendations.

This chapter elaborates on the relevance and value of the findings from this dissertation and addresses valorization opportunities for the individual, the health professional, and research.

\section{Relevance}

Findings from our formative work are consistent with results from existing research regarding co-occurring treatment non-adherence in patients with T2DM. Results from our qualitative formative research study already suggested that non-adherence to treatment behaviors often co-occurs. Our baseline measurement from the conducted trial confirmed this finding and showed that, on average, patients could improve on three of the four treatment recommendations that were examined. Former research already showed an increasing negative trend towards co-occurring treatment non-adherence. Only 3.5\% of the studied patients with T2DM adhered to all five key recommendations related to the treatment of T2DM, while this percentage was almost three times as high in prior observations (25). Results from former research demonstrated that almost $85 \%$ could improve adherence to two or more recommendations, while earlier research showed that this percentage was near $75 \%$, also indicating a negative trend. With an increasing prevalence of T2DM, more patients are at risk for the multifaceted adverse consequences related to the disease and treatment nonadherence. Moreover, the increasingly common co-occurrence of treatment non-adherence may result in greater adversity on health and related outcomes (198). In the first place for the patient, but also from a societal and economical perspective, it is therefore evident to target improvements in patients' adherence and by doing so from a multi-behavior perspective.

Current Dutch guidelines with regard to diabetes care describe that discussing and promoting treatment adherence should be one of the standard discussion topics in encounters between 
the patient and the health professional (14). Increasing care demands, time constraints, and scarce encounters between patient and professional may however impede addressing treatment adherence to a sufficient extent, as well as the adequate application of multiple factors which seem to determine successful treatment adherence. Findings reported in this dissertation, but also lessons learned from earlier work in which nurses reported to struggle providing effective lifestyle counselling, ask for new avenues to address and improve treatment adherence (63). Discussing treatment adherence in medical encounters could serve as a starting point for extended treatment adherence enhancing activities outside the healthcare setting. An example of such an activity could be to partly outsource the process of assessing and improving treatment adherence to the context of the Internet. The added value for the professional is that this could be useful given the presumed time and know-how shortage on how to promote health behavior change. In addition, the professional could be updated on the progress of the behavior change of the patient periodically, thereby integrating the online intervention into the healthcare context. For instance, like in the My Diabetes Profile program, the professional could get access to a summary of the activities a patient has done in the program and the progress in improving treatment adherence. Moreover, this way, professionals could devote more attention to other, more acute discussion topics such as the patient's latest blood glucose levels. Especially as traditionally healthcare systems were developed to deal with acute conditions rather than chronic illnesses, it is not surprising that professionals experience difficulties and barriers in discussing and promoting treatment adherence amongst their chronic ill patients (105). For the patient, personalized advice as provided in the eHealth context, has the advantage that it can be accessed at any time and in a desired pace.

The effectiveness study in the second part of this dissertation suggested that such an intervention delivered through the Internet, i.e. the newly developed My Diabetes Profile program, can be an effective way to improve patients' overall adherence. Besides the general attributes of Internet interventions, such as its broad reach, constant accessibility, and potential to temper pressure on health care systems, the program also has its unique features. Compared to the vast majority of existing eHealth interventions targeting adherence improvements in patients with T2DM, the My Diabetes Profile program provided patients with personally relevant tailored content, applied a sound theoretical base, incorporated largely visual content, and was developed with input from patients and professionals. These attributes are well-known to enhance the likelihood of a program to be successful in improving treatment adherence, and it is likely that its application has resulted in the positive effects of My Diabetes Profile. However, future studies could investigate if indeed the application of the known success factors led to the positive results as observed in the effectiveness evaluation. 


\section{Innovation}

The My Diabetes Profile shows some level of innovation in relation to existing computertailored interventions, as well as in its strategy to improve patient adherence. Firstly, the field of computer tailoring technology and eHealth has evolved tremendously in recent decades. First generation tailored strategies applied print materials to bring about individualized health messages. In recent years, the Internet has increasingly emerged as mode for the delivery of tailored interventions to improve for instance adherence to treatment strategies, referred to as computer-tailoring technology $(72,81,239)$. Most previous web-based computer-tailored interventions focusing on T2DM treatment adherence did not apply a solid theoretical framework, were generally mono-behavior oriented, included little interactive content, and were largely text-based (154). The My Diabetes Profile program not only builds on a solid theoretical framework, i.e. the I-Change Model, it also aims to improve patient adherence from a multi-behavior perspective, applying strategies towards both healthy lifestyle adaptations and medical intervention adherence. As disease and glycemic control are impacted by all these treatment behaviors, and as treatment non-adherence often co-occurs, it seems that a multi-behavior strategy is most relevant. The interactivity of the My Diabetes Profile program is reflected in the content that is assessment based, tailored to the behavior change phase an individual is in, and segmented to a high, medium, or low score on related determinant scales. In addition, at the level of beliefs, tailored advice is provided to e.g. persuade an individual of a certain advantage of carrying out a behavior, or to strengthen self-efficacy in a particular difficult situation. Advice is provided instantly and accessible at a preferred pace, contrary to e.g. the computer-tailored letters sent within weeks to months following the assessment in earlier work (239). Many of the interactive pathways applied in My Diabetes Profile are delivered through video-tailored advice. People with a low education level, which is common in people with T2DM, may be less text-oriented which in turn could inhibit the use and effectiveness of such programs (240). Video-tailoring is an innovative mode to bring about health promotion messages that can deliver content in a more interactive way compared to text-tailoring. Further, video-tailoring for example enables to demonstrate skills to patients, reduces cognitive processing of information, and can lead to better appreciation and attention, compared to text-tailored content (241). Moreover, it is suggested that the application of video-messages in order to deliver health promotion interventions, may particularly suit low-educated patients, given their generally low-literate skills and difficulty of translating abstract text into concrete actions (242-244).

Secondly, rather than aiming to reach the recommended norms of those behaviors, as has been the case in previous similar work $(52,245)$, our program aimed for improvements in all of our targeted behaviors, i.e. physical activity, medication adherence, and a decrease in caloric intake from unhealthy snacks, from a continuous perspective. An increase from two to twenty-nine minutes of daily physical activity would not have counted as a successful behavior change in programs that aim for patients reaching the norm, while an increase from 
twenty-nine minutes of daily physical activity to thirty minutes would actually have. Recent Dutch and international guideline targets on physical activity conclude that any increase, and especially an increase in activity levels from zero to any activity, can have an enormous impact on health and related outcomes (191). Moreover, if a certain norm was reached but an in- or decrease was still possible, our program aimed for these improvements as e.g. it is known that additional activity beyond the norm of 150 weekly minutes is desirable. Therefore, if feasible, future interventions should carefully consider whether aiming to reach the norm and only reaching the norm is sufficient to grasp behavioral improvements below or beyond those norms, and rather aim for continuous improvements instead. Moreover, by promoting small steps, it is also easier to provide reinforcing feedback in the challenging trajectory of behavior change among patients with T2DM (116).

\section{Target groups}

\section{Patients with type 2 diabetes mellitus}

First and foremost, the results of this dissertation are of great value for the initial target group of the intervention, i.e. Dutch patients with T2DM. It is conceivable that patients with T2DM beyond the age restrictions applied in the trial could benefit from the intervention, as the presence of T2DM is increasingly common in those aged $<40$ and $>70$ (246). However, if the program would be made available for youngsters, young adults, or elderly above the age of 70 with T2DM, it is likely that the program requires some form of adaptation towards specific beliefs and target goals of those groups. Similar formative research as reported in the early chapters of this dissertation could yield information on if and how the program should be adapted to align with needs and salient beliefs present in those target groups (247). In addition, the program modules targeting improvements in healthy lifestyle behaviors could be of interest for people suffering from other lifestyle related diseases or for people at risk of developing T2DM. As the onset as well as the treatment of T2DM largely involves energy balance-related behaviors such as physical activity and dietary choices, those at risk could contribute to a decreased chance of developing T2DM by improving healthy lifestyle adherence. Internationally, the program could be of impact as T2DM is known to be a global epidemic, however, again the context and content should be fine-tuned to those countries or regions it is implemented to.

\section{Health professionals}

Another relevant target group to potentially benefit from current findings are health professionals, especially those directly involved in diabetes care of patients, i.e. nurses and physicians. First, a discrepancy seems to exist in perceptions of patients and their health professionals with regard to whether patients adhere to distinct treatment recommendations (28). Therefore, it is at least of relevance to raise awareness in professionals and patient that their views on patient adherence may deviate and that advice provided by health professionals 
accordingly may be perceived as irrelevant by patients. Second, the My Diabetes Profile program includes a certain level of blended care as the program is proposed to patients in medical encounters, and as professionals get access to a summary of patients' activity in the program. In blended care interventions, therapeutic guidance is complemented with online care. A combination is recommended, as stand-alone online interventions struggle with disappointing compliance rates $(248,249)$. However, it remains unclear what the best way and quantity is to integrate these two delivery modes. A trend is observable towards a declining share of the health professional in interventions that have been developed at our university in recent years that aimed for self-management support or improvements in healthy lifestyles. On the contrary, technology is more and more integrated in routine care to complement or partly replace the work conducted by nurses. In the work of Koelewijn and colleagues, primary care nurses were trained to deliver risk and motivational counseling to improve patients' lifestyle related to cardiovascular risk management (250). It is concluded that nurses should support patients in their attempts to improve their lifestyle, but that only nurse-support does not seem to be sufficient (251). A project to improve the daily functioning of patients with T2DM by the implementation of a nurse-led intervention for integrated self-management support in routine care, showed limited effects. A process-evaluation showed that practice nurses felt restricted in time and expertise to adopt the intervention, and patients did not expect a discussion about psychosocial problems but rather saw their nurse as a specialist regarding the biomedical management of their T2DM (252). More recent work was pointed at assisting practice nurses on how to best deliver goal setting and action planning with regard to selfmanagement support in chronically ill patients. Nurses seemed to experience difficulties in setting goals and action plans with regard to self-management support. Their focus was merely on solving medical problems, and patients were characterized as rather passive collaborators in this process, as has been established earlier $(28,253)$. Furthermore, nurses indicated to struggle to integrate the approach in routine care and to apply it in a tailored way, and characterized the process as time consuming and complex (254). Verwey and colleagues aimed to improve physical activity levels in patients with T2DM and acknowledged that mere advice from a practice nurse would not result in significant improvements in activity levels. Patients visit their practice nurse a couple times a year, while behavior change may require day-to-day attention. Therefore, a technological support tool was developed for patients to complement the process of risk communication, motivational interviewing and goal setting by nurses (237). A summary of activity levels monitored by the tool was sent to the practice nurse automatically, similar as in the My Diabetes Profile program. The feedback tool, if embedded into a counseling protocol, was an effective instrument to improve physical activity levels of patients with T2DM, while mere counseling was not effective (255). In sum, it seems that efforts of nurses alone are often not enough to realize sufficient behavior change in patients, party through time constraints, the infrequent occurrence of medical encounters, know-how insufficiencies, and passive patient involvement. Especially in attempts to improve multiple behaviors, the integration of routine care with online interventions where patients can access advice on a daily basis seems evident. The counseling process, for example 
risk communication, motivational messages and goal-setting and action planning, can be outsourced to the context of the Internet. Future studies need to explore how to best attune the face-to-face counseling process with tailored eHealth methods.

\section{Researchers}

Results from this dissertation also provide relevant knowledge and opportunities for researchers. An important step in developing health promotion programs is the phase of testing the program on its effectiveness and if results are positive, subsequently the dissemination of successful programs in order to increase its exposure and impact (256). In the trial that was conducted as part of the effectiveness study, patients were recruited by their health professional, i.e. practice nurses and diabetes nurses in the primary and secondary care setting. Beforehand, all nurses intended to recruit at least eight patients with T2DM to our trial. However, trial data showed a broad range of recruitment success of nurses, ranging from 0-32 recruited patients. It is widely acknowledged that for instance for health promotion behaviors, a positive intention towards behavior change does not warrant subsequent success (56). It seems that recruitment behavior of nurses in our trial was subject to a similar intentionbehavior gap, and that nurses' recruitment behavior in general follows similar pathways as how health behavior can be explained in the I-Change Model. Therefore, researchers need to be aware that not all nurses who intent to recruit patients will actually do so, and may dropout during the recruitment period.

Researchers may, however, be able to influence the process of recruitment by health professionals. After conducting a qualitative exploration of factors influencing recruitment success among nurses it seems that there is a wide variety of factors that influences recruitment success including, and foremost, cognitions of recruiters themselves seem to have an impact on the outcome. A personalized approach seems to be the primary method once a nurse has expressed the willingness to participate. For instance, in some nurses the expressed intention is followed-up by clear action plans, coping strategies towards barriers, and goals regarding the intended number of recruited patients. Others seem to have expressed their willingness without actually having sufficient study and trial knowledge and sufficient motivation to recruit patients. It is yet to become clear how researchers can optimally support recruiters through a personalized approach to stimulate their recruitment efforts and related outcomes. For now, it is suggested that researchers could assess recruiters' trial and study knowledge, motivation and willingness to recruit study subjects, and align their efforts in assisting recruiters towards their needs, in order to increase the likelihood of recruitment being successful. More research in this area, however, is required to substantiate the suggested initiatives. 


\section{Feasibility and dissemination of My Diabetes Profile}

Program impact not merely depends on its effectiveness, but also on its reach and implementation by its users (257). In order to be of impact, the reach of the successful My Diabetes Program should be increased beyond the trial setting in which its effectiveness was examined. One needs to be aware that this is the first version of the My Diabetes Profile program, and although overall effectiveness has been shown, further refinement may be needed. First, largely the program was developed with input from local stakeholders, i.e. patients and professionals were interviewed as described in chapter 2, and a program committee was formed prior to program development to accumulate input. Although to some extent attention has been payed to co-creation, it is relevant to involve national stakeholders such as patient associations, health insurance companies and national diabetes federations to further attune the program and to create a supportive base for the program. As described earlier, further research should also be conducted on how to optimally integrate the program with face-to-face counseling.

Subsequently, this information may help researchers to optimize the program and ultimately, a My Diabetes Profile program 2.0 version could be tested in a similar trial to examine if enhanced success is achieved, before widespread implementation is initiated. Subsequently, if researchers would aim for broad implementation of the My Diabetes Profile program, implementation challenges may need to be explored. For instance, researchers could explore if the delivery format of the My Diabetes Profile program through nurses in consultations is in fact a feasible delivery strategy, and if and how the eHealth program can be integrated in the multitude of information systems used in Dutch healthcare. Moreover, prolonged use as well as long-term effects and cost-effectiveness need to be researched to increase the marketing of the program. In sum, it can be concluded that the My Diabetes Profile program in its current version was an effective and feasible program to improve overall treatment adherence in patients with T2DM. However, the program may need further refinement before widespread implementation is initiated.

\section{Lifestyle medicine and prevention}

The aims of My Diabetes Profile program are consistent with the vision of the Dutch ministry of Public Health, Welfare and Sports as stated in the recently published Dutch prevention agreement (258). Maintaining or improving healthy lifestyles are not only part of the prevention and treatment of T2DM, but are also urgent for Dutch society as in 2017 almost half of the people aged $\geq 20$ was at least overweight. This number of overweight and obese people is increasing rapidly, which in turn are important risk factors for the onset of T2DM. Energy balance related behaviors, such as healthy dietary choices and sufficient physical activity, are key behaviors in the prevention of obesity and T2DM. Lifestyle medicine and 
disease prevention have therefore gained ground in recent years, and are elaborated in the Dutch prevention agreement. However, few attention has been payed to eHealth as method to bring about improvements in healthy lifestyle behaviors. In addition, many initiatives stated in the agreement to target a complex problem of overweight and obesity, focus on the environment and to a smaller extent on cognitions, i.e. personal determinants such as the individual's knowledge and motivation. The My Diabetes Profile program showed that eHealth as medium and intervening in the personal determinants was a successful strategy to decrease caloric intake from unhealthy snacks. Given the expectation that by 2040 over 60\% of the Dutch population will at least be overweight as well as the expected increase of T2DM, My Diabetes Profile can be a valuable tool to support patients with T2DM and potentially those with overweight, in effective, personalized and broad reach healthy lifestyle changes. 
References 


\section{References}

1. Federation ID. IDF Diabetes Atlas 8th Edition 2017 [cited 2019 March, 20th]. Available from: https:// www.idf.org/e-library/epidemiology-research/diabetes-atlas/134-idf-diabetes-atlas-8th-edition. html.

2. RIVM. Prevalentie diabetes naar leeftijd en geslacht 2019 [cited 2019 March, 20th]. Available from: https://www.volksgezondheidenzorg.info/onderwerp/diabetes-mellitus/cijfers-context/huidigesituatie\#node-prevalentie-diabetes-naar-leeftijd-en-geslacht.

3. Holman N, Young B, Gadsby R. Current prevalence of Type 1 and Type 2 diabetes in adults and children in the UK. Diabetic medicine : a journal of the British Diabetic Association. 2015;32(9):1119-20.

4. Idenburg PJ, van Schaik M, de Weerdt I. Diagnose diabetes 2025: over de toekomst van de Nederlandse diabeteszorg. Schiedam: Scriptum; 2012.

5. Basu S, Yoffe P, Hills N, Lustig RH. The relationship of sugar to population-level diabetes prevalence: an econometric analysis of repeated cross-sectional data. PloS one. 2013;8(2):e57873.

6. Association AD. Statistics About Diabetes: Overall Numbers, Diabetes and Prediabetes 2018 [cited 201924 June]. Available from: http://www.diabetes.org/diabetes-basics/statistics/.

7. van't Riet E, Schram MT, Abbink EJ, Admiraal WM, Dijk-Schaap MW, Holleman F, et al. The Diabetes Pearl: Diabetes biobanking in The Netherlands. BMC public health. 2012;12:949.

8. Carstensen B, Jorgensen ME, Friis S. The epidemiology of diabetes and cancer. Curr Diab Rep. 2014;14(10):535.

9. Lu FP, Lin KP, Kuo HK. Diabetes and the risk of multi-system aging phenotypes: a systematic review and meta-analysis. PloS one. 2009;4(1):e4144.

10. Roy T, Lloyd CE. Epidemiology of depression and diabetes: a systematic review. Journal of affective disorders. 2012;142 Suppl:S8-21.

11. Volksgezondheidszorg.info. Kosten van zorg voor diabetes mellitus 2019 [cited 2019 July 4th]. Available from: https://www.volksgezondheidenzorg.info/onderwerp/diabetes-mellitus/kosten/ kosten\#node-kosten-van-zorg-voor-diabetes-mellitus.

12. Volksgezondheidszorg.info. Sterfte diabetes naar leeftijd en geslacht 2019 [cited 2019 July 4th]. Available from: https://www.volksgezondheidenzorg.info/onderwerp/diabetes-mellitus/cijferscontext/sterfte.

13. NHG. NHG-Standaard Diabetes mellitus type 22019 [cited 201924 June]. Available from: https:// www.nhg.org/standaarden/volledig/nhg-standaard-diabetes-mellitus-type-2.

14. Federatie ND. De NDF Zorgstandaard diabetes Type 2 volwassenen 2013 [cited 2017 October 12th]. Available from: http://www.zorgstandaarddiabetes.nl/type-2/.

15. Garcia-Perez LE, Alvarez M, Dilla T, Gil-Guillen V, Orozco-Beltran D. Adherence to therapies in patients with type 2 diabetes. Diabetes therapy : research, treatment and education of diabetes and related disorders. 2013;4(2):175-94.

16. Holman RR, Paul SK, Bethel MA, Matthews DR, Neil HA. 10-year follow-up of intensive glucose control in type 2 diabetes. The New England journal of medicine. 2008;359(15):1577-89.

17. Funnell MM, Anderson RM. Empowerment and self-management of diabetes. Clinical Diabetes. 2004;22(3):123-7. 
18. Asche C, LaFleur J, Conner C. A review of diabetes treatment adherence and the association with clinical and economic outcomes. Clinical therapeutics. 2011;33(1):74-109.

19. Ho PM, Rumsfeld JS, Masoudi FA, McClure DL, Plomondon ME, Steiner JF, et al. Effect of medication nonadherence on hospitalization and mortality among patients with diabetes mellitus. Archives of internal medicine. 2006;166(17):1836-41.

20. Lorber D. Importance of cardiovascular disease risk management in patients with type 2 diabetes mellitus. Diabetes, metabolic syndrome and obesity : targets and therapy. 2014;7:169-83.

21. Barr EL, Zimmet PZ, Welborn TA, Jolley D, Magliano DJ, Dunstan DW, et al. Risk of cardiovascular and all-cause mortality in individuals with diabetes mellitus, impaired fasting glucose, and impaired glucose tolerance: the Australian Diabetes, Obesity, and Lifestyle Study (AusDiab). Circulation. 2007;116(2):151-7.

22. Stratton IM, Adler Al, Neil HA, Matthews DR, Manley SE, Cull CA, et al. Association of glycaemia with macrovascular and microvascular complications of type 2 diabetes (UKPDS 35): prospective observational study. Bmj. 2000;321(7258):405-12.

23. Nelson KM, Reiber G, Boyko EJ, Nhanes, III. Diet and exercise among adults with type 2 diabetes: findings from the third national health and nutrition examination survey (NHANES III). Diabetes care. 2002;25(10):1722-8.

24. Morrato EH, Hill JO, Wyatt HR, Ghushchyan V, Sullivan PW. Physical activity in U.S. adults with diabetes and at risk for developing diabetes, 2003. Diabetes care. 2007;30(2):203-9.

25. King DE, Mainous AG, 3rd, Carnemolla M, Everett CJ. Adherence to healthy lifestyle habits in US adults, 1988-2006. The American journal of medicine. 2009;122(6):528-34.

26. Resnick HE, Foster GL, Bardsley J, Ratner RE. Achievement of American Diabetes Association clinical practice recommendations among U.S. adults with diabetes, 1999-2002: the National Health and Nutrition Examination Survey. Diabetes care. 2006;29(3):531-7.

27. Janevic MR, McLaughlin SJ, Connell CM. Overestimation of physical activity among a nationally representative sample of underactive individuals with diabetes. Medical care. 2012;50(5):441-5.

28. Vluggen S, Hoving C, Schaper NC, de Vries H. Exploring beliefs on diabetes treatment adherence among Dutch type 2 diabetes patients and healthcare providers. Patient education and counseling. 2018;101(1):92-8.

29. Katz LL, Anderson BJ, McKay SV, Izquierdo R, Casey TL, Higgins LA, et al. Correlates of Medication Adherence in the TODAY Cohort of Youth With Type 2 Diabetes. Diabetes care. 2016;39(11):1956-62.

30. Krass I, Schieback P, Dhippayom T. Adherence to diabetes medication: a systematic review. Diabetic medicine : a journal of the British Diabetic Association. 2015;32(6):725-37.

31. Kok G, Gottlieb NH, Peters GJY, Mullen PD, Parcel GS, Ruiter RAC, et al. A taxonomy of behaviour change methods: an Intervention Mapping approach. Health Psychology Review. 2016;10(3):297312.

32. Jaam M, Awaisu A, Ibrahim Ml, Kheir N. Synthesizing and Appraising the Quality of the Evidence on Factors Associated with Medication Adherence in Diabetes: A Systematic Review of Systematic Reviews. Value Health Reg Issues. 2017;13:82-91.

33. Sabaté E. Adherence to long-term therapies: evidence for action. Geneva, Switzerland: World Health Organization; 2003. 
34. Tuerk PW, Mueller M, Egede LE. Estimating physician effects on glycemic control in the treatment of diabetes: methods, effects sizes, and implications for treatment policy. Diabetes care. 2008;31(5):86973.

35. Bandura A. Social foundations of thought and action: A social cognitive perspecive. Englewood Cliffs, NJ: Princeton-Hall; 1986.

36. Prochaska JO, DiClemente CC. Stages and processes of self-change of smoking: toward an integrative model of change. J Consult Clin Psychol. 1983;51(3):390-5.

37. Janz NK, Becker MH. The Health Belief Model: a decade later. Health Educ Q. 1984;1 1 (1):1-47.

38. Ajzen I. The Theory of Planned Behavior. Organizational Behavior and Human Decision Processes. 1991;50(2):179-211.

39. Fishbein M, Ajzen I. Belief, Attitude, Intention, and Behavior: An Introduction to Theory and Research. Philosophy and Rhetoric. 1977;10(2):130-2.

40. Plotnikoff RC, Lippke S, Courneya K, Birkett N, Sigal R. Physical activity and diabetes: an application of the theory of planned behaviour to explain physical activity for Type 1 and Type 2 diabetes in an adult population sample. Psychol Health. 2010;25(1):7-23.

41. Dehghani-Tafti A, Mazloomy Mahmoodabad SS, Morowatisharifabad MA, Afkhami Ardakani M, Rezaeipandari H, Lotfi MH. Determinants of Self-Care in Diabetic Patients Based on Health Belief Model. Glob J Health Sci. 2015;7(5):33-42.

42. Sniehotta FF, Presseau J, Araujo-Soares V. Time to retire the theory of planned behaviour. Health Psychol Rev. 2014;8(1):1-7.

43. Vries H. An Integrated Approach for Understanding Health Behavior; The I-Change Model as an Example. Psychology and Behavioral Science International Journal. 2017;2(2).

44. McEachan RRC, Conner M, Taylor NJ, Lawton RJ. Prospective prediction of health-related behaviours with the Theory of Planned Behaviour: a meta-analysis. Health Psychology Review. 2011;5(2):97-144.

45. Kasten $\mathrm{S}$, van Osch $\mathrm{L}$, Candel $\mathrm{M}$, de Vries $\mathrm{H}$. The influence of pre-motivational factors on behavior via motivational factors: a test of the I-Change model. BMC Psychol. 2019;7(1):7.

46. Sheeran P, Webb TL. The Intention-Behavior Gap. Soc Personal Psychol. 2016;10(9):503-18.

47. Sniehotta FF, Schwarzer R, Scholz U, Schuz B. Action planning and coping planning for long-term lifestyle change: Theory and assessment. Eur J Soc Psychol. 2005;35(4):565-76.

48. Sniehotta FF, Scholz U, Schwarzer R. Action plans and coping plans for physical exercise: A longitudinal intervention study in cardiac rehabilitation. British journal of health psychology. 2006;11:23-37.

49. Kasten S, van Osch L, Eggers SM, de Vries H. From action planning and plan enactment to fruit consumption: moderated mediation effects. BMC public health. 2017;17.

50. de Vries H, Eggers SM, Bolman C. The role of action planning and plan enactment for smoking cessation. BMC public health. 2013;13.

51. Walthouwer MJ, Oenema A, Lechner L, de Vries H. Comparing a Video and Text Version of a WebBased Computer-Tailored Intervention for Obesity Prevention: A Randomized Controlled Trial. Journal of medical Internet research. 2015;17(10):e236. 
52. Schulz DN, Kremers SP, Vandelanotte C, van Adrichem MJ, Schneider F, Candel MJ, et al. Effects of a web-based tailored multiple-lifestyle intervention for adults: a two-year randomized controlled trial comparing sequential and simultaneous delivery modes. Journal of medical Internet research. 2014;16(1):e26.

53. Lechner $L$, De Vries $H$. Starting participation in an employee fitness program: attitudes, social influence, and self-efficacy. Preventive medicine. 1995;24(6):627-33.

54. Bandura A. Self-efficacy: toward a unifying theory of behavioral change. Psychol Rev. 1977;84(2):191215.

55. de Vries H, Dijkstra M, Kuhlman P. Self-efficacy: the third factor besides attitude and subjective norm as a predictor of behavioural intentions. Health education research. 1988;3(3):273-82.

56. Sniehotta FF, Scholz U, Schwarzer R. Bridging the intention-behaviour gap: Planning, self-efficacy, and action control in the adoption and maintenance of physical exercise. Psychology \& Health. 2005;20(2):143-60.

57. Schuz B, Sniehotta FF, Mallach N, Wiedemann AU, Schwarzer R. Predicting transitions from preintentional, intentional and actional stages of change. Health education research. 2009;24(1):6475.

58. Green LW, Kreuter MW. Health program planning: An educational and ecological approach (4th ed.). New York: McGraw Hill Professional; 2005.

59. Bartholomew LK, Parcel GS, Kok G, Gottlieb NH, Fernandez ME. Planning Health Promotion Programs: An Intervention Mapping Approach (3rd Edition). San Francisco: Jossey-Bass; 2011.

60. Glasgow RE, Vogt TM, Boles SM. Evaluating the public health impact of health promotion interventions: the RE-AIM framework. American journal of public health. 1999;89(9):1322-7.

61. Mensing C, Boucher J, Cypress M, Weinger K, Mulcahy K, Barta P, et al. National standards for diabetes self-management education. Diabetes care. 2007;30 Suppl 1:S96-S103.

62. Duclos M, Dejager S, Postel-Vinay N, di Nicola S, Quere S, Fiquet B. Physical activity in patients with type 2 diabetes and hypertension--insights into motivations and barriers from the MOBILE study. Vasc Health Risk Manag. 2015;11:361-71.

63. Jansink R, Braspenning J, van der Weijden T, Elwyn G, Grol R. Primary care nurses struggle with lifestyle counseling in diabetes care: a qualitative analysis. BMC family practice. 2010;11:41.

64. Jarvis J, Skinner TC, Carey ME, Davies MJ. How can structured self-management patient education improve outcomes in people with type 2 diabetes? Diabetes, obesity \& metabolism. 2010;12(1):129.

65. Williams JL, Walker RJ, Smalls BL, Campbell JA, Egede LE. Effective interventions to improve medication adherence in Type 2 diabetes: a systematic review. Diabetes management. 2014;4(1):2948.

66. Vermeire E, Wens J, Van Royen P, Biot Y, Hearnshaw H, Lindenmeyer A. Interventions for improving adherence to treatment recommendations in people with type 2 diabetes mellitus. The Cochrane database of systematic reviews. 2005(2):CD003638.

67. Sapkota S, Brien JA, Greenfield JR, Aslani P. A Systematic Review of Interventions Addressing Adherence to Anti-Diabetic Medications in Patients with Type 2 Diabetes--Components of Interventions. PloS one. 2015;10(6):e0128581. 
68. Fan LF, Sidani S. Effectiveness of Diabetes Self-management Education Intervention Elements: A Meta-analysis. Canadian Journal of Diabetes. 2009;33(1):18-26.

69. Eysenbach G. What is e-health? Journal of medical Internet research. 2001;3(2).

70. Ramadas A, Quek KF, Chan CK, Oldenburg B. Web-based interventions for the management of type 2 diabetes mellitus: a systematic review of recent evidence. International journal of medical informatics. 2011;80(6):389-405.

71. Cotterez AP, Durant N, Agne AA, Cherrington AL. Internet interventions to support lifestyle modification for diabetes management: a systematic review of the evidence. J Diabetes Complications. 2014;28(2):243-51.

72. Kohl LF, Crutzen R, de Vries NK. Online Prevention Aimed at Lifestyle Behaviors: A Systematic Review of Reviews. Journal of medical Internet research. 2013;15(7):71-83.

73. de Vries H, Kremers SP, Smeets T, Brug J, Eijmael K. The effectiveness of tailored feedback and action plans in an intervention addressing multiple health behaviors. American journal of health promotion : AJHP. 2008;22(6):417-25.

74. Pal K, Eastwood SV, Michie S, Farmer AJ, Barnard ML, Peacock R, et al. Computer-based diabetes self-management interventions for adults with type 2 diabetes mellitus. The Cochrane database of systematic reviews. 2013(3):CD008776.

75. Smit ES, Evers SM, de Vries H, Hoving C. Cost-effectiveness and cost-utility of Internet-based computer tailoring for smoking cessation. Journal of medical Internet research. 2013;15(3):e57.

76. Griffiths F, Lindenmeyer A, Powell J, Lowe P, Thorogood M. Why are health care interventions delivered over the internet? A systematic review of the published literature. Journal of medical Internet research. 2006;8(2):e10.

77. Clark M, Hampson SE, Avery L, Simpson R. Effects of a tailored lifestyle self-management intervention in patients with type 2 diabetes. British journal of health psychology. 2004;9(Pt 3):365-79.

78. Boudreau F, Godin G, Poirier P. Effectiveness of a computer-tailored print-based physical activity intervention among French Canadians with type 2 diabetes in a real-life setting. Health education research. 2011;26(4):573-85.

79. Glasgow RE, Kurz D, King D, Dickman JM, Faber AJ, Halterman E, et al. Outcomes of minimal and moderate support versions of an internet-based diabetes self-management support program. Journal of general internal medicine. 2010;25(12):1315-22.

80. Otten W, Rövekamp T, Keer M, Graaf Ad, Keulen Hv. Mogelijkheden voor webbased ondersteuning bij zelfmanagement van Type 2 Diabetes Mellitus. Leiden: TNO; 2015.

81. de Vries H, Brug J. Computer-tailored interventions motivating people to adopt health promoting behaviours: introduction to a new approach. Patient education and counseling. 1999;36(2):99-105.

82. Kreuter MW, Strecher VJ, Glassman B. One size does not fit all: the case for tailoring print materials. Annals of behavioral medicine : a publication of the Society of Behavioral Medicine. 1999;21(4):27683.

83. Kreuter MW, Wray RJ. Tailored and targeted health communication: Strategies for enhancing information relevance. American Journal of Health Behavior. 2003;27:S227-S32. 
84. French DP, Wade AN, Farmer AJ. Predicting self-care behaviours of patients with type 2 diabetes: the importance of beliefs about behaviour, not just beliefs about illness. Journal of psychosomatic research. 2013;74(4):327-33.

85. Ajzen I. Martin Fishbein's Legacy:The Reasoned Action Approach. Ann Am Acad Polit Ss. 2012;640:1127.

86. Moreau M, Gagnon MP, Boudreau F. Development of a fully automated, web-based, tailored intervention promoting regular physical activity among insufficiently active adults with type 2 diabetes: integrating the I-change model, self-determination theory, and motivational interviewing components. JMIR research protocols. 2015;4(1):e25.

87. Hariton E, Locascio JJ. Randomised controlled trials - the gold standard for effectiveness research Study design: randomised controlled trials. Bjog-Int J Obstet Gy. 2018;125(13):1716-

88. Campbell M, Fitzpatrick R, Haines A, Kinmonth AL, Sandercock P, Spiegelhalter D, et al. Framework for design and evaluation of complex interventions to improve health. Brit Med J. 2000;321(7262):6946.

89. Fukui T, Rahman M, Shimbo T, Morita S, Sakamoto J. Recruitment of patients for a clinical trial: factors on the physician side and reasons on the patient side. Intern Med. 2006;45(8):511-4.

90. Page MJ, French SD, McKenzie JE, O'Connor DA, Green SE. Recruitment difficulties in a primary care cluster randomised trial: investigating factors contributing to general practitioners' recruitment of patients. BMC medical research methodology. 2011;11:35.

91. Newington L, Metcalfe A. Factors influencing recruitment to research: qualitative study of the experiences and perceptions of research teams. BMC medical research methodology. 2014;14:10.

92. Tooher RL, Middleton PF, Crowther CA. A thematic analysis of factors influencing recruitment to maternal and perinatal trials. BMC Pregnancy Childbirth. 2008;8:36.

93. Carter RE. Application of stochastic processes to participant recruitment in clinical trials. Control Clin Trials. 2004;25(5):429-36.

94. Pringle M, Churchill R. Randomised controlled trials in general practice. Bmj. 1995;311(7017):1382-3.

95. Watson JM, Torgerson DJ. Increasing recruitment to randomised trials: a review of randomised controlled trials. BMC medical research methodology. 2006;6:34.

96. Ngune I, Jiwa M, Dadich A, Lotriet J, Sriram D. Effective recruitment strategies in primary care research: a systematic review. Qual Prim Care. 2012;20(2):115-23.

97. RIVM. Nationaal Kompas Volksgezondheid, versie 4.17: Diabetes Mellitus 2014 [Available from: http://www.nationaalkompas.nl/gezondheid-en-ziekte/ziekten-en-aandoeningen/endocrienevoedings-en-stofwisselingsziekten-en-immuniteitsstoornissen/diabetes-mellitus/.

98. Federation ID. Diabetes Atlas, sixth edition 2013 [Available from: http://www.idf.org/sites/default/ files/EN_6E_Atlas_Full_0.pdf.

99. Swinburn BA, Sacks G, Hall KD, McPherson K, Finegood DT, Moodie ML, et al. The global obesity pandemic: shaped by global drivers and local environments. Lancet. 2011;378(9793):804-14.

100. Newman S, Steed, L. \& Mulligan, K. Chronic Physical Illness: Self-management and Behavioural Inteventions. Maidenhead: Open University Press; 2009. 
101. Vijan S, Stuart NS, Fitzgerald JT, Ronis DL, Hayward RA, Slater S, et al. Barriers to following dietary recommendations in Type 2 diabetes. Diabetic medicine : a journal of the British Diabetic Association. 2005;22(1):32-8.

102. Rubin RR. Adherence to pharmacologic therapy in patients with type 2 diabetes mellitus. The American journal of medicine. 2005;118 Suppl 5A:27S-34S.

103. Cramer JA. A systematic review of adherence with medications for diabetes. Diabetes care. 2004;27(5):1218-24.

104. Funnell MM \& Anderson RM. Empowerment and Self-Management of Diabetes. Clinical Diabetes. 2004;22(3):123-7.

105. Wagner EH, Austin BT, Davis C, Hindmarsh M, Schaefer J, Bonomi A. Improving chronic illness care: translating evidence into action. Health affairs. 2001;20(6):64-78.

106. Delamater AM. Improving patient adherence. Clinical Diabetes. 2006;24:71-7.

107. Francis JJ, Johnston M, Robertson C, Glidewell L, Entwistle V, Eccles MP, et al. What is an adequate sample size? Operationalising data saturation for theory-based interview studies. Psychol Health. 2010;25(10):1229-45.

108. Vries H, Mesters I, van de Steeg H, Honing C. The general public's information needs and perceptions regarding hereditary cancer: an application of the Integrated Change Model. Patient education and counseling. 2005;56(2):154-65.

109. Ajzen I. The Theory of Planned Behavior. Organizational Behavior and Human Decision Processes. 1991;50:179-211.

110. de Vries H, Mesters I, Riet JV, Willems K, Reubsaet A. Motives of Belgian adolescents for using sunscreen: the role of action plans. Cancer epidemiology, biomarkers \& prevention : a publication of the American Association for Cancer Research, cosponsored by the American Society of Preventive Oncology. 2006;15(7):1360-6.

111. Berkson J. A System of Codification of Medical Diagnoses for Application to Punch Cards, With a Plan of Operation. Am J Public Health Nations Health. 1936;26(6):606-12.

112. Mortelmans D. Kwalitatieve analyse met Nvivo. Leuven/Den Haag: Acco; 2011.

113. Altman DG. Practical Statistics for Medical Research London: Chapman \& Hall/CRC; 1991.

114. Inzucchi SE, Bergenstal RM, Buse JB, Diamant M, Ferrannini E, Nauck M, et al. Management of hyperglycaemia in type 2 diabetes: a patient-centered approach. Position statement of the American Diabetes Association (ADA) and the European Association for the Study of Diabetes (EASD). Diabetologia. 2012;55(6):1577-96.

115. Montori VM, Gafni A, Charles C. A shared treatment decision-making approach between patients with chronic conditions and their clinicians: the case of diabetes. Health expectations : an international journal of public participation in health care and health policy. 2006;9(1):25-36.

116. DeWalt DA, Davis TC, Wallace AS, Seligman HK, Bryant-Shilliday B, Arnold CL, et al. Goal setting in diabetes self-management: taking the baby steps to success. Patient education and counseling. 2009;77(2):218-23.

117. Naik AD, Palmer N, Petersen NJ, Street RL, Jr., Rao R, Suarez-Almazor $M$, et al. Comparative effectiveness of goal setting in diabetes mellitus group clinics: randomized clinical trial. Archives of internal medicine. 2011;171(5):453-9. 
118. Heisler M, Vijan S, Anderson RM, Ubel PA, Bernstein SJ, Hofer TP. When do patients and their physicians agree on diabetes treatment goals and strategies, and what difference does it make? Journal of general internal medicine. 2003;18(11):893-902.

119. Larkin AT, Hoffman C, Stevens A, Douglas A, Bloomgarden Z. Determinants of adherence to diabetes treatment. Journal of diabetes. 2015;7(6):864-71.

120. McSharry J, McGowan L, Farmer AJ, French DP. Perceptions and experiences of taking oral medications for the treatment of Type 2 diabetes mellitus: a systematic review and meta-synthesis of qualitative studies. Diabetic medicine : a journal of the British Diabetic Association. 2016;33(10):1330-8.

121. Mishali M, Omer H, Heymann AD. The importance of measuring self-efficacy in patients with diabetes. Family practice. 2011;28(1):82-7.

122. Clifford S, Perez-Nieves M, Skalicky AM, Reaney M, Coyne KS. A systematic literature review of methodologies used to assess medication adherence in patients with diabetes. Current medical research and opinion. 2014;30(6):1071-85.

123. Nguyen TMU, La Caze A, Cottrell N. What are validated self-report adherence scales really measuring?: a systematic review. British journal of clinical pharmacology. 2014;77(3):427-45.

124. Kleppe M, Lacroix J, Ham J, Midden C. The development of the ProMAS: a Probabilistic Medication Adherence Scale. Patient preference and adherence. 2015;9:355-67.

125. Horne R, Hankins M, Jenkins R. The Satisfaction with Information about Medicines Scale (SIMS): a new measurement tool for audit and research. Qual Health Care. 2001;10(3):135-40.

126. Morisky DE, Green LW, Levine DM. Concurrent and predictive validity of a self-reported measure of medication adherence. Medical care. 1986;24(1):67-74.

127. Horne R, Weinman J. Self-regulation and self-management in asthma: Exploring the role of illness perceptions and treatment beliefs in explaining non-adherence to preventer medication. Psychology \& Health. 2002;17(1):17-32.

128. MacLaughlin EJ, Raehl CL, Treadway AK, Sterling TL, Zoller DP, Bond CA. Assessing medication adherence in the elderly: which tools to use in clinical practice? Drugs Aging. 2005;22(3):231-55.

129. Lehmann A, Aslani P, Ahmed R, Celio J, Gauchet A, Bedouch P, et al. Assessing medication adherence: options to consider. International journal of clinical pharmacy. 2014;36(1):55-69.

130. Chao J, Nau DP, Aikens JE, Taylor SD. The mediating role of health beliefs in the relationship between depressive symptoms and medication adherence in persons with diabetes. Research in social \& administrative pharmacy: RSAP. 2005;1(4):508-25.

131. Jannuzzi FF, Rodrigues RC, Cornelio ME, Sao-Joao TM, Gallani MC. Beliefs related to adherence to oral antidiabetic treatment according to the Theory of Planned Behavior. Rev Lat Am Enfermagem. 2014;22(4):529-37.

132. Faul F, Erdfelder E, Lang AG, Buchner A. G*Power 3: a flexible statistical power analysis program for the social, behavioral, and biomedical sciences. Behav Res Methods. 2007;39(2):175-91.

133. Koster ES, Philbert D, Winters NA, Bouvy ML. Adolescents' inhaled corticosteroid adherence: the importance of treatment perceptions and medication knowledge. J Asthma. 2015;52(4):431-6.

134. Voncken-Brewster V, Tange H, Moser A, Nagykaldi Z, de Vries H, van der Weijden T. Integrating a tailored e-health self-management application for chronic obstructive pulmonary disease patients into primary care: a pilot study. BMC family practice. 2014;15:4. 
135. Downey RG, King C. Missing data in Likert ratings: A comparison of replacement methods. J Gen Psychol. 1998;125(2):175-91.

136. Heinze G, Wallisch C, Dunkler D. Variable selection - A review and recommendations for the practicing statistician. Biometrical J. 2018;60(3):431-49.

137. Hinkle DE, Wiersma W, Jurs SG. Applied Statistics for the Behavioral Sciences (5th ed.). Boston: Houghton Mifflin; 2003.

138. Osterberg L, Blaschke T. Adherence to medication. The New England journal of medicine. 2005;353(5):487-97.

139. Hansen RA, Farley JF, Droege M, Maciejewski ML. A retrospective cohort study of economic outcomes and adherence to monotherapy with metformin, pioglitazone, or a sulfonylurea among patients with type 2 diabetes mellitus in the United States from 2003 to 2005. Clinical therapeutics. 2010;32(7):1308-19.

140. Kreyenbuhl J, Dixon LB, McCarthy JF, Soliman S, Ignacio RV, Valenstein M. Does Adherence to Medications for Type 2 Diabetes Differ Between Individuals With Vs Without Schizophrenia? Schizophrenia Bull. 2010;36(2):428-35.

141. Hertz RP, Unger AN, Lustik MB. Adherence with pharmacotherapy for type 2 diabetes: A retrospective cohort study of adults with employer-sponsored health insurance. Clinical therapeutics. 2005;27(7):1064-73.

142. Guenette L, Lauzier S, Guillaumie L, Giguere G, Gregoire JP, Moisan J. Patients' beliefs about adherence to oral antidiabetic treatment: a qualitative study. Patient preference and adherence. 2015;9:413-20.

143. DiMatteo MR. Variations in patients' adherence to medical recommendations: a quantitative review of 50 years of research. Medical care. 2004;42(3):200-9.

144. Kirkman MS, Rowan-Martin MT, Levin R, Fonseca VA, Schmittdiel JA, Herman WH, et al. Determinants of Adherence to Diabetes Medications: Findings From a Large Pharmacy Claims Database. Diabetes care. 2015;38(4):604-9.

145. Chew BH, Hassan NH, Sherina MS. Determinants of medication adherence among adults with type 2 diabetes mellitus in three Malaysian public health clinics: a cross-sectional study. Patient preference and adherence. 2015;9:639-48.

146. Jin J, Sklar GE, Min Sen Oh V, Chuen Li S. Factors affecting therapeutic compliance: A review from the patient's perspective. Therapeutics and clinical risk management. 2008;4(1):269-86.

147. Mann DM, Ponieman D, Leventhal H, Halm EA. Predictors of adherence to diabetes medications: the role of disease and medication beliefs. Journal of behavioral medicine. 2009;32(3):278-84.

148. Rogers RW. A Protection Motivation Theory of Fear Appeals and Attitude Change1. J Psychol. 1975;91(1):93-114.

149. Vervloet M, Spreeuwenberg P, Bouvy ML, Heerdink ER, de Bakker DH, van Dijk L. Lazy sunday afternoons: the negative impact of interruptions in patients' daily routine on adherence to oral antidiabetic medication. A multilevel analysis of electronic monitoring data. Eur J Clin Pharmacol. 2013;69(8):1599-606.

150. Farmer A, Kinmonth AL, Sutton S. Measuring beliefs about taking hypoglycaemic medication among people with Type 2 diabetes. Diabetic Med. 2006;23(3):265-70. 
151. Wroe AL. Intentional and unintentional nonadherence: a study of decision making. Journal of behavioral medicine. 2002;25(4):355-72.

152. Federation ID. IDF Diabetes Atlas, 7th edn. Brussels, Belgium2015 [cited 2017 April 6th]. Available from: http://www.diabetesatlas.org.

153. DIEP. Digitale Leefstijlcoach 2005-2015 [Available from: http://www.diep.info/diabetes-digitaleleefstijlcoach

154. Walthouwer MJ, Oenema A, Lechner L, de Vries H. Use and Effectiveness of a Video- and Text-Driven Web-Based Computer-Tailored Intervention: Randomized Controlled Trial. Journal of medical Internet research. 2015;17(9):e222.

155. Stanczyk N, Bolman C, van Adrichem M, Candel M, Muris J, de Vries H. Comparison of text and video computer-tailored interventions for smoking cessation: randomized controlled trial. Journal of medical Internet research. 2014;16(3):e69.

156. Havelock RG. The Utilization of Educational Research and Development Journal of Educational Technology. 1971(2):84-97.

157. Vandelanotte C. Are simultaneously delivered health behavior change interventions the way of the future?: a comment on King et al. Annals of behavioral medicine : a publication of the Society of Behavioral Medicine. 2013;46(2):133-4.

158. Gezondheidsraad. Beweegrichtlijnen 2017. Den Haag; Gezondheidsraad 2017; publicatienr. $2017 / 08$.

159. GoAnimate. Video Maker 2017 [cited 2018 February 27th]. Available from: https://goanimate.com/ videomaker.

160. Strobelt H, Oelke D, Kwon BC, Schreck T, Pfister H. Guidelines for Effective Usage of Text Highlighting Techniques. IEEE Trans Vis Comput Graph. 2016;22(1):489-98.

161. Dijkstra A, De Vries H. The development of computer-generated tailored interventions. Patient education and counseling. 1999;36(2):193-203.

162. Jaspers MW. A comparison of usability methods for testing interactive health technologies: methodological aspects and empirical evidence. International journal of medical informatics. 2009;78(5):340-53.

163. Fry JP, Neff RA. Periodic prompts and reminders in health promotion and health behavior interventions: systematic review. Journal of medical Internet research. 2009;11(2):e16.

164. Schneider F, de Vries H, Candel M, van de Kar A, van Osch L. Periodic email prompts to re-use an internet-delivered computer-tailored lifestyle program: influence of prompt content and timing. Journal of medical Internet research. 2013;15(1):e23.

165. Prochaska JJ, Velicer WF, Nigg CR, Prochaska JO. Methods of quantifying change in multiple risk factor interventions. Preventive medicine. 2008;46(3):260-5.

166. Wendel-Vos GC, Schuit AJ, Saris WH, Kromhout D. Reproducibility and relative validity of the short questionnaire to assess health-enhancing physical activity. J Clin Epidemiol. 2003;56(12):1163-9.

167. Verhoeven AA, Adriaanse MA, Evers C, de Ridder DT. The power of habits: unhealthy snacking behaviour is primarily predicted by habit strength. British journal of health psychology. 2012;17(4):758-70. 
168. Voedingscentrum. Omgaan met producten buiten de schijf van vijf 2017 [cited 2017 December 7th]. Available from: http://www.voedingscentrum.nl/nl/gezond-eten-met-de-schijf-van-vijf/ omgaan-met-producten-buiten-de-schijf-van-vijf.aspx.

169. Tommelein E, Mehuys E, Van Tongelen I, Brusselle G, Boussery K. Accuracy of the Medication Adherence Report Scale (MARS-5) as a quantitative measure of adherence to inhalation medication in patients with COPD. The Annals of pharmacotherapy. 2014;48(5):589-95.

170. Eysenbach G. The law of attrition. Journal of medical Internet research. 2005;7(1):e11.

171. Ma Y, Olendzki BC, Li W, Hafner AR, Chiriboga D, Hebert JR, et al. Seasonal variation in food intake, physical activity, and body weight in a predominantly overweight population. European journal of clinical nutrition. 2006;60(4):519-28.

172. Foster JM, Sawyer SM, Smith L, Reddel HK, Usherwood T. Barriers and facilitators to patient recruitment to a cluster randomized controlled trial in primary care: lessons for future trials. BMC medical research methodology. 2015;15:18.

173. Ross S, Grant A, Counsell C, Gillespie W, Russell I, Prescott R. Barriers to participation in randomised controlled trials: a systematic review. J Clin Epidemiol. 1999;52(12):1143-56.

174. Foy R, Parry J, Duggan A, Delaney B, Wilson S, Lewin-van den Broek N, et al. How evidence based are recruitment strategies to randomized controlled trials in primary care? Experience from seven studies. Family practice. 2003;20(1):83-92.

175. Vluggen S, Hoving C, Schaper NC, de Vries H. A web-based program to improve treatment adherence in patients with type 2 diabetes: Development and study protocol. Contemp Clin Trials. 2018;74:38-45.

176. Strauss A, Corbin J. Basics of qualitative research: Techniques and procedures for developing grounded theory (2nd ed.). Thousand Oaks, CA: Sage; 1998.

177. Thomas DR. A general inductive approach for analyzing qualitative evaluation data. Am J Eval. 2006;27(2):237-46.

178. Sadler GR, Lantz JM, Fullerton JT, Dault Y. Nurses' unique roles in randomized clinical trials. Journal of Professional Nursing. 1999;15(2):106-15.

179. Donovan JL, Peters TJ, Noble S, Powell P, Gillatt D, Oliver SE, et al. Who can best recruit to randomized trials? Randomized trial comparing surgeons and nurses recruiting patients to a trial of treatments for localized prostate cancer (the ProtecT study). J Clin Epidemiol. 2003;56(7):605-9.

180. Mason V, Shaw A, Wiles N, Mulligan J, Peters T, Sharp D, et al. GPs' experiences of primary care mental health research: a qualitative study of the barriers to recruitment. Family practice. 2007;24(5):518-25.

181. McAlister A, Perry C, Parcel G. How individuals, environments, and health behaviors interact: Social Cognitive Theory. In K. Glanz, B. Rimer, \& K. Viswanath (Eds.). Health behavior and health education (4th ed, pp 169-188). San Francisco: Jossey-Bass; 2008.

182. Fletcher B, Gheorghe A, Moore D, Wilson S, Damery S. Improving the recruitment activity of clinicians in randomised controlled trials: a systematic review. BMJ open. 2012;2(1):e000496.

183. Spaar A, Frey M, Turk A, Karrer W, Puhan MA. Recruitment barriers in a randomized controlled trial from the physicians' perspective - A postal survey. BMC medical research methodology. 2009;9. 
184. Dormandy E, Kavalier F, Logan J, Harris H, Ishmael N, Marteau TM, et al. Maximising recruitment and retention of general practices in clinical trials: a case study. The British journal of general practice : the journal of the Royal College of General Practitioners. 2008;58(556):759-66, i-ii.

185. Pearl A, Wright S, Gamble G, Doughty R, Sharpe N. Randomised trials in general practice--a New Zealand experience in recruitment. The New Zealand medical journal. 2003;116(1186):U681.

186. Bell-Syer SEM, Moffett JAK. Recruiting patients to randomized trials in primary care: principles and case study. Family practice. 2000;17(2):187-91.

187. Gollwitzer PM. Implementation intentions - Strong effects of simple plans. American Psychologist. 1999:54(7):493-503.

188. Bower P, Wallace P, Ward E, Graffy J, Miller J, Delaney B, et al. Improving recruitment to health research in primary care. Family practice. 2009;26(5):391-7.

189. Sapkota S, Brien JA, Greenfield J, Aslani P. A systematic review of interventions addressing adherence to anti-diabetic medications in patients with type 2 diabetes--impact on adherence. PloS one. 2015;10(2):e0118296.

190. Ainsworth BE, Haskell WL, Whitt MC, Irwin ML, Swartz AM, Strath SJ, et al. Compendium of physical activities: an update of activity codes and MET intensities. Med Sci Sports Exerc. 2000;32(9 Suppl):S498-504.

191. Weggemans RM, Backx FJG, Borghouts L, Chinapaw M, Hopman MTE, Koster A, et al. The 2017 Dutch Physical Activity Guidelines. The international journal of behavioral nutrition and physical activity. 2018;15(1):58.

192. Voedingscentrum. Hoeveel calorieën zitten erin? 2019 [cited 2019 February 18th]. Available from: https://www.voedingscentrum.nl/nl/service/vraag-en-antwoord/gezonde-voeding-envoedingsstoffen/hoeveel-calorieen-zitten-erin-.aspx.

193. Drake BF, Quintiliani LM, Sapp AL, Li Y, Harley AE, Emmons KM, et al. Comparing strategies to assess multiple behavior change in behavioral intervention studies. Translational behavioral medicine. 2013;3(1):114-21.

194. Sullivan GM, Feinn R. Using Effect Size—or Why the P Value Is Not Enough. Journal of Graduate Medical Education. 2012;4(3):279-82.

195. Sullivan GM, Feinn R. Using Effect Size-or Why the P Value Is Not Enough. J Grad Med Educ. 2012:4(3):279-82.

196. Heinze G, Dunkler D. Five myths about variable selection. Transpl Int. 2017;30(1):6-10.

197. Spring B, Schneider K, McFadden HG, Vaughn J, Kozak AT, Smith M, et al. Multiple behavior changes in diet and activity: a randomized controlled trial using mobile technology. Archives of internal medicine. 2012;172(10):789-96.

198. Prochaska JJ, Spring B, Nigg CR. Multiple health behavior change research: an introduction and overview. Preventive medicine. 2008;46(3):181-8.

199. Thabane L, Mbuagbaw L, Zhang S, Samaan Z, Marcucci M, Ye C, et al. A tutorial on sensitivity analyses in clinical trials: the what, why, when and how. BMC medical research methodology. 2013;13:92.

200. Graham JW, Donaldson SI. Evaluating interventions with differential attrition: the importance of nonresponse mechanisms and use of follow-up data. J Appl Psychol. 1993;78(1):119-28. 
201. Blankers M, Smit ES, van der Pol P, de Vries H, Hoving C, van Laar M. The Missing=Smoking Assumption: A Fallacy in Internet-Based Smoking Cessation Trials? Nicotine \& Tobacco Research. 2016;18(1):25-33.

202. Glasgow RE, Kurz D, King D, Dickman JM, Faber AJ, Halterman E, et al. Twelve-month outcomes of an Internet-based diabetes self-management support program. Patient education and counseling. 2012;87(1):81-92.

203. Soler RE, Leeks KD, Razi S, Hopkins DP, Griffith M, Aten A, et al. A systematic review of selected interventions for worksite health promotion. The assessment of health risks with feedback. Am J Prev Med. 2010;38(2 Suppl):S237-62.

204. Lorig K, Ritter PL, Laurent DD, Plant K, Green M, Jernigan VB, et al. Online diabetes self-management program: a randomized study. Diabetes care. 2010;33(6):1275-81.

205. McKay HG, King D, Eakin EG, Seeley JR, Glasgow RE. The diabetes network internet-based physical activity intervention: a randomized pilot study. Diabetes care. 2001;24(8):1328-34.

206. Health Council of the Netherlands. Dutch physical activity guidelines 2017. The Hague: Health Council of the Netherlands; 2017 [cited 201829 June]. publication no. 2017/08e]. Available from: https://www.gezondheidsraad.nl/documenten/adviezen/2017/08/22/beweegrichtlijnen-2017.

207. Nicolaou M, Gademan MG, Snijder MB, Engelbert RH, Dijkshoorn H, Terwee CB, et al. Validation of the SQUASH Physical Activity Questionnaire in a Multi-Ethnic Population: The HELIUS Study. PloS one. 2016;11(8):e0161066.

208. Seligman HK, Wallace AS, DeWalt DA, Schillinger D, Arnold CL, Shilliday BB, et al. Facilitating behavior change with low-literacy patient education materials. Am J Health Behav. 2007;31 Suppl 1:S69-78.

209. Schmeichel BJ, Vohs K. Self-Affirmation and Self-Control: Affirming Core Values Counteracts Ego Depletion. Journal of Personality and Social Psychology. 2009;96(4):770-82.

210. Finne E, Englert C, Jekauc D. On the importance of self-control strength for regular physical activity. Psychol Sport Exerc. 2019;43:165-71.

211. van Osch L, Lechner L, Reubsaet A, De Vries H. From theory to practice: An explorative study into the instrumentality and specificity of implementation intentions. Psychology \& Health. 2010;25(3):351-64.

212. Malpass A, Andrews R, Turner KM. Patients with Type 2 Diabetes experiences of making multiple lifestyle changes: a qualitative study. Patient education and counseling. 2009;74(2):258-63.

213. Eysenbach G. The Law of Attrition. Journal of medical Internet research. 2005;7(1).

214. Stellefson M, Chaney B, Barry AE, Chavarria E, Tennant B, Walsh-Childers K, et al. Web 2.0 Chronic Disease Self-Management for Older Adults: A Systematic Review. Journal of medical Internet research. 2013;15(2).

215. Van der Mispel C, Poppe L, Crombez G, Verloigne M, De Bourdeaudhuij I. A Self-Regulation-Based eHealth Intervention to Promote a Healthy Lifestyle: Investigating User and Website Characteristics Related to Attrition. Journal of medical Internet research. 2017;19(7).

216. Vermeire E, Hearnshaw H, Van Royen P, Denekens J. Patient adherence to treatment: three decades of research. A comprehensive review. Journal of clinical pharmacy and therapeutics. 2001;26(5):331-42.

217. Munshi MN, Slyne C, Greenberg JM, Greaves T, Lee A, Carl S, et al. Nonadherence to Insulin Therapy Detected by Bluetooth-Enabled Pen Cap Is Associated With Poor Glycemic Control. Diabetes care. 2019;42(6):1129-31. 
218. Ley SH, Hamdy O, Mohan V, Hu FB. Prevention and management of type 2 diabetes: dietary components and nutritional strategies. Lancet. 2014;383(9933):1999-2007.

219. Hu FB. Globalization of diabetes: the role of diet, lifestyle, and genes. Diabetes care. 2011;34(6):124957.

220. Fayet-Moore F, Peters V, McConnell A, Petocz P, Eldridge AL. Weekday snacking prevalence, frequency, and energy contribution have increased while foods consumed during snacking have shifted among Australian children and adolescents: 1995, 2007 and 2011-12 National Nutrition Surveys. Nutr J. 2017;16.

221. Vahasarja K, Salmela S, Villberg J, Rintala P, Vanhala M, Saaristo T, et al. Perceived Sufficiency of Physical Activity Levels Among Adults at High Risk of Type 2 Diabetes: The FIN-D2D Study. International Journal of Behavioral Medicine. 2014;21(1):99-108.

222. Kemper H, Ooijendijk, WTM, Stiggelbout, M. Consensus over de Nederlandse Norm voor Gezond bewegen. Tijdschrift voor Gezondheidswetenschappen. 2000;78:180-3.

223. Jette M, Sidney K, Blumchen G. Metabolic equivalents (METS) in exercise testing, exercise prescription, and evaluation of functional capacity. Clin Cardiol. 1990;13(8):555-65.

224. Stolpe S, Kroes MA, Webb N, Wisniewski T. A Systematic Review of Insulin Adherence Measures in Patients with Diabetes. Journal of managed care \& specialty pharmacy. 2016;22(11):1224-46.

225. Wens J, Vermeire E, Royen PV, Sabbe B, Denekens J. GPs' perspectives of type 2 diabetes patients' adherence to treatment: A qualitative analysis of barriers and solutions. BMC family practice. 2005;6(1):20.

226. Bandura A. Self-Efficacy Mechanism in Human Agency. American Psychologist. 1982;37(2):122-47.

227. Affendi I, Nor M, Normi M, Mohd M, Noor L, Sabtuah R, et al. Association between Self-Efficacy and Health Behaviour in Disease Control: A Systematic Review. Global Journal of Health Science. 2018;10(1):18-36.

228. Chin MH, Cook S, Jin L, Drum ML, Harrison JF, Koppert J, et al. Barriers to providing diabetes care in community health centers. Diabetes care. 2001;24(2):268-74.

229. Lambe B, Collins C. A qualitative study of lifestyle counselling in general practice in Ireland. Family practice. 2010;27(2):219-23.

230. Hebert ET, Caughy MO, Shuval K. Primary care providers' perceptions of physical activity counselling in a clinical setting: a systematic review. Br J Sports Med. 2012;46(9):625-31.

231. Talboom-Kamp EPWA, Verdijk NA, Kasteleyn MJ, Numans ME, Chavannes NH. From chronic disease management to person-centered eHealth; a reviewon the necessity for blended care. Clinical eHealth. 2018;1(1):3-7.

232. van Ommen B, Wopereis S, van Empelen P, van Keulen HM, Otten W, Kasteleyn M, et al. From Diabetes Care to Diabetes Cure-The Integration of Systems Biology, eHealth, and Behavioral Change. Front Endocrinol (Lausanne). 2017;8:381.

233. Vluggen S, Hoving C, Schaper NC, de Vries H. Exploring beliefs on diabetes treatment adherence among Dutch type 2 diabetes patients and healthcare providers. Patient education and counseling. 2017.

234. Lie SS, Karlsen B, Oord ER, Graue M, Oftedal B. Dropout From an eHealth Intervention for Adults With Type 2 Diabetes: A Qualitative Study. Journal of medical Internet research. 2017;19(5):e187. 
235. Barbeau EM, Wallace L, Lederman R, Lightman N, Stoddard A, Sorensen G. Recruiting small manufacturing worksites that employ multiethnic, low-wage workforces into a cancer prevention research trial. Preventing chronic disease. 2004;1(3):A04.

236. Emmons KM, Stoddard AM, Fletcher R, Gutheil C, Suarez EG, Lobb R, et al. Cancer prevention among working class, multiethnic adults: results of the healthy directions-health centers study. American journal of public health. 2005;95(7):1200-5.

237. Verwey R, van der Weegen S, Spreeuwenberg M, Tange H, van der Weijden T, de Witte L. Upgrading physical activity counselling in primary care in the Netherlands. Health Promotion International. 2016;31(2):344-54.

238. Sanyal C, Stolee P, Juzwishin D, Husereau D. Economic evaluations of eHealth technologies: A systematic review. PloS one. 2018;13(6):e0198112.

239. van Stralen MM, Kok G, de Vries H, Mudde AN, Bolman C, Lechner L. The Active plus protocol: systematic development of two theory- and evidence-based tailored physical activity interventions for the over-fifties. BMC public health. 2008;8:399.

240. Sacerdote C, Ricceri F, Rolandsson O, Baldi I, Chirlaque MD, Feskens E, et al. Lower educational level is a predictor of incident type 2 diabetes in European countries: The EPIC-InterAct study. International Journal of Epidemiology. 2012;41(4):1162-73.

241. Alley S, Jennings C, Persaud N, Plotnikoff RC, Horsley M, Vandelanotte C. Do personally tailored videos in a web-based physical activity intervention lead to higher attention and recall? - an eyetracking study. Front Public Health. 2014;2:13.

242. Gerber BS, Brodsky IG, Lawless KA, Smolin LI, Arozullah AM, Smith EV, et al. Implementation and evaluation of a low-literacy diabetes education computer multimedia application. Diabetes care. 2005;28(7):1574-80.

243. Kandula NR, Nsiah-Kumi PA, Makoul G, Sager J, Zei CP, Glass S, et al. The relationship between health literacy and knowledge improvement after a multimedia type 2 diabetes education program. Patient education and counseling. 2009;75(3):321-7.

244. Stanczyk NE, Bolman C, Muris JW, de Vries H. Study protocol of a Dutch smoking cessation e-health program. BMC public health. 2011;11:847.

245. van Stralen MM, de Vries H, Mudde AN, Bolman C, Lechner L. The long-term efficacy of two computer-tailored physical activity interventions for older adults: main effects and mediators. Health psychology : official journal of the Division of Health Psychology, American Psychological Association. 2011;30(4):442-52.

246. Volksgezondheidszorg.nl. Prevalentie diabetes naar leeftijd en geslacht 2019 [cited 2019 August 26th]. Available from: https://www.volksgezondheidenzorg.info/onderwerp/diabetes-mellitus/ cijfers-context/huidige-situatie\#!node-prevalentie-diabetes-naar-leeftijd-en-geslacht.

247. Kok G, Gottlieb NH, Peters GJ, Mullen PD, Parcel GS, Ruiter RA, et al. A taxonomy of behaviour change methods: an Intervention Mapping approach. Health Psychol Rev. 2016;10(3):297-312.

248. Kelders SM, Kok RN, Ossebaard HC, Van Gemert-Pijnen JEWC. Persuasive System Design Does Matter: A Systematic Review of Adherence to Web-Based Interventions. Journal of medical Internet research. 2012;14(6):17-40. 
249. Kloek C, Bossen D, de Bakker DH, Veenhof C, Dekker J. Blended Interventions to Change Behavior in Patients With Chronic Somatic Disorders: Systematic Review. Journal of medical Internet research. 2017;19(12).

250. Koelewijn-van Loon MS, van der Weijden T, Ronda G, van Steenkiste B, Winkens B, Elwyn G, et al. Improving lifestyle and risk perception through patient involvement in nurse-led cardiovascular risk management: A cluster-randomized controlled trial in primary care. Preventive medicine. 2010;50(1-2):35-44.

251. Koelewijn-van Loon MS. Gezamenlijke besluitvorming en preventie van hartvaatziekten: een methode voor praktijkondersteuners. Kwaliteit in Zorg. 2011 (2):27-9.

252. van Dijk-de Vries A, van Bokhoven MA, de Jong S, Metsemakers JF, Verhaak PF, van der Weijden T, et al. Patients' readiness to receive psychosocial care during nurse-led routine diabetes consultations in primary care: A mixed methods study. Int J Nurs Stud. 2016;63:58-64.

253. Lenzen SA, Stommel W, Daniels R, van Bokhoven MA, van der Weijden T, Beurskens A. Ascribing patients a passive role: Conversation analysis of practice nurses' and patients' goal setting and action planning talk. Research in Nursing \& Health. 2018;41(4):389-97.

254. Lenzen SA, Daniels R, van Bokhoven MA, van der Weijden T, Beurskens A. Development of a conversation approach for practice nurses aimed at making shared decisions on goals and action plans with primary care patients. BMC health services research. 2018;18(1):891.

255. van der Weegen S, Verwey R, Spreeuwenberg M, Tange H, van der Weijden T, de Witte L. It's LiFe! Mobile and Web-Based Monitoring and Feedback Tool Embedded in Primary Care Increases Physical Activity: A Cluster Randomized Controlled Trial. Journal of medical Internet research. 2015;17(7):e184.

256. King DK, Glasgow RE, Leeman-Castillo B. Reaiming RE-AIM: using the model to plan, implement, and evaluate the effects of environmental change approaches to enhancing population health. American journal of public health. 2010;100(11):2076-84.

257. Abrams DB, Orleans CT, Niaura RS, Goldstein MG, Prochaska JO, Velicer W. Integrating individual and public health perspectives for treatment of tobacco dependence under managed health care: a combined stepped-care and matching model. Annals of behavioral medicine : a publication of the Society of Behavioral Medicine. 1996;18(4):290-304.

258. Ministerie van Volksgezondheid WeS. Nationaal Preventieakkoord. Naar een gezonder Nederland 2018 [cited 2019 August 28th]. Available from: https://www.rijksoverheid.nl/onderwerpen/ gezondheid-en-preventie/documenten/convenanten/2018/11/23/nationaal-preventieakkoord. 
Summary

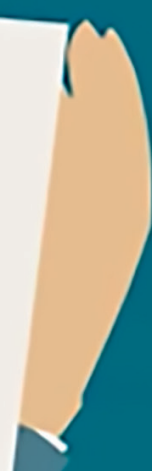




\section{Summary}

The main aim of this dissertation is to describe the development and effectiveness of the eHealth program, My Diabetes Profile, which aimed to improve adherence of patients with T2DM to core treatment behaviors, i.e. improving physical activity levels, oral hypoglycemic agent $(\mathrm{OHA})$ adherence, insulin therapy adherence, and decreasing caloric intake from unhealthy snacks.

Chapter 1 provides a general introduction including background information on diabetes mellitus, its consequences, management strategies, and treatment adherence. Diabetes Mellitus is a progressive chronic disease characterized by hyperglycemia and the inability of the body to maintain a favorable glucose metabolism. By 2045, worldwide over 600 million people will be affected by diabetes, and nine in every ten people of those will have type 2 diabetes mellitus. Treatment strategies consist of increasing physical activity levels, improving dietary patterns, and if applicable adherence to medical behaviors such as OHA taking and insulin therapy. Patient adherence poses a serious challenge, non-adherence co-occurs across treatment behaviors, and non-adherence is associated with considerable individual, societal, and economic consequences. In that light, it is evident to improve adherence and to explore avenues on how to achieve improvements in patients' treatment adherence. In addition, the introduction provides insight in the theoretical framework applied in this dissertation, and discusses computer-tailoring technology as medium to improve treatment adherence in patients with diabetes.

Chapter 2 describes a qualitative inquiry among patients with type 2 diabetes and relevant health professionals involved in the management of diabetes. The study aimed to explore the scope of non-adherence, to explore salient personal beliefs associated with treatment adherence, and to explore if and how success factors for treatment adherence are applied in diabetes consultations. Results demonstrated that treatment adherence to healthy lifestyle and medical behaviors was suboptimal. It seems that for some treatment behaviors, patients had more knowledge and were more aware of risks compared to other behaviors. Health professionals and patients reported many advantages and no disadvantages of adherence to healthy lifestyle behaviors. Both advantages and disadvantages were reported for medical behavior adherence. Self-efficacy of patients to adhere to treatment behaviors seemed to be lower in situations when routine was compromised; similar situations were identified for distinct treatment behaviors. Last, success factors for treatment adherence were insufficiently applied in consultations. Health professionals reported that scarce, busy, and brief consultations may impede the application of such success factors. In conclusion, patient adherence is suboptimal, non-adherence co-occurs across behaviors, beliefs have been identified to target in interventions, and new avenues need to be explored to address success factors for treatment adherence. 
Chapter 3 describes a longitudinal study that aimed to identify psychological predictors of adherence to OHAs and to compare the prevalence of adherence between two self-report adherence instruments. At baseline, we assessed OHA adherence using the ProMAS and MARS-5, socio-cognitive determinants, and demographics. At follow-up, the ProMAS was used as outcome measure, on which socio-cognitive determinants and demographics were regressed using multiple linear regression analysis. With currently advised and widely applied cut-off points of $\geq 15$ for the ProMAS and $\geq 23$ for the MARS-5, $47 \%$ and $90 \%$ adhered to their $\mathrm{OHAs}$ respectively. Data of the ProMAS were more in line with objectively collected data with regard to the percentage of patients that could be considered adherent. Consistent predictors of better adherence were a low education, lower severity perceptions, and higher selfefficacy and intention. After correcting for baseline adherence, a low education and higher self-efficacy remained significant predictors. In sum, the ProMAS seems better equipped to deal with pitfalls present in available self-report measures. Suboptimal adherence stresses the need for effective adherence improving interventions. Interventions should particularly target higher-educated patients and patients with low self-efficacy in situations out of daily routine.

Chapter 4 describes the development of the My Diabetes Profile program as well as a protocol for the conduct of a randomized controlled trial to examine its effectiveness. Results from chapters two and three were complemented with knowledge accumulated from previously developed computer-tailored programs and input gathered from a program committee. Together these sources yielded input for the development of the eHealth program My Diabetes Profile. Consequently, the program addressed treatment non-adherence to healthy lifestyle and medical behaviors, and aligned with the needs and desires of our target group and others involved in the committee. In addition, the program incorporated success factors for treatment adherence as identified from the literature, i.e. a solid theoretical base, interactive content and feedback tailored to relevant phases and determinants of behavior change, visual support, and the possibility to monitor adherence.

Chapter 5 discussed the evaluation that was conducted regarding the recruitment of patients with type 2 diabetes by nurses to our randomized controlled trial. The aim of this study was to map the number of patients recruited per nurse and how this total came about during the recruitment period, and to explore factors that influenced nurses' their recruitment success. Nurses' recruitment success and the course of recruitment success were derived from trial data (NTR6840). Based on the standardized total number of recruited patients, nurses were categorized either as non-, low-, medium-, or high-recruiter. Ninety-six nurses participated in our trial and recruited a mean of seven patients (range 0-32), which was slightly lower than the minimum of eight patients we aimed for. Fifteen nurses did not recruit any patient. Most patients were recruited close to recruitment onset. Nurses who did not recruit patients close to recruitment onset, ultimately showed no recruitment success. Data showed a relative high early recruitment success that progressively declined over time; high-recruiters were generally successful throughout the entire recruitment period, while low-recruiters mostly in the early 
months. Telephone interviews among a subset of nurses per group revealed that recruitment barriers and facilitators emerged in the area of organizational, study, patient and especially recruiter characteristics. Contrary to non- and low-recruiters, medium- and high-recruiters reported more in-depth knowledge about the study and trial requirements, expressed more personal participation benefits and fewer barriers, and applied more recruitment activities, reminders, and barrier-focused coping strategies. To conclude, early recruitment success should be encouraged, and a personalized approach may facilitate recruiters to become and remain successful.

Chapter 6 describes the results of the randomized controlled trial that was conducted to examine the effectiveness of the My Diabetes Profile program in comparison to a waiting list control arm. Intervention effects were examined on overall and separate treatment behaviour adherence. Sixty percent of those patients who completed the baseline assessment were retained after follow-up, which was a promising result for eHealth interventions. Overall treatment adherence improved significantly in those patients allocated to the intervention, reflected by an overall small to medium effect size $(d=.24)$. With regard to separate treatment behaviors, a significant decrease was observed in caloric intake from unhealthy snacks $(d=.38)$. Small to medium positive intervention effects were observed for adherence to $\mathrm{OHA}(d=.18)$ and insulin therapy ( $d=.28$ ), however, these effects were not statistically significant, potentially due to a lack of power in these medical behaviors. As the My Diabetes Profile program applied several success factors for eHealth interventions identified from the literature, i.e. a theoretical base, tailored content and a multi-behavior approach, this may have contributed to the success of the intervention. eHealth has shown to be an effective avenue to improve treatment adherence in patients with T2DM. Future research should investigate how widescale implementation could best be shaped and if and how the integration of eHealth in usual care could be of additional value.

Finally, chapter 7 provides a general discussion and conclusion based on the studies presented throughout the dissertation. The My Diabetes Profile program was effective in improving treatment adherence of patients with T2DM. However, several research areas have been touched upon which require further research. For instance, the long-term effects of the program on treatment behavior adherence and related outcomes remain unknown. Furthermore, the cost-effectiveness evaluation, process-evaluation, and optimal delivery format for wide-spread implementation should be explored in order to provide entries to market the program, refine the program, and increase the program's impact. Last, the integration of eHealth interventions in usual care, i.e. blended care, has shown to be beneficial, but requires further investigation for the My Diabetes Profile program. 


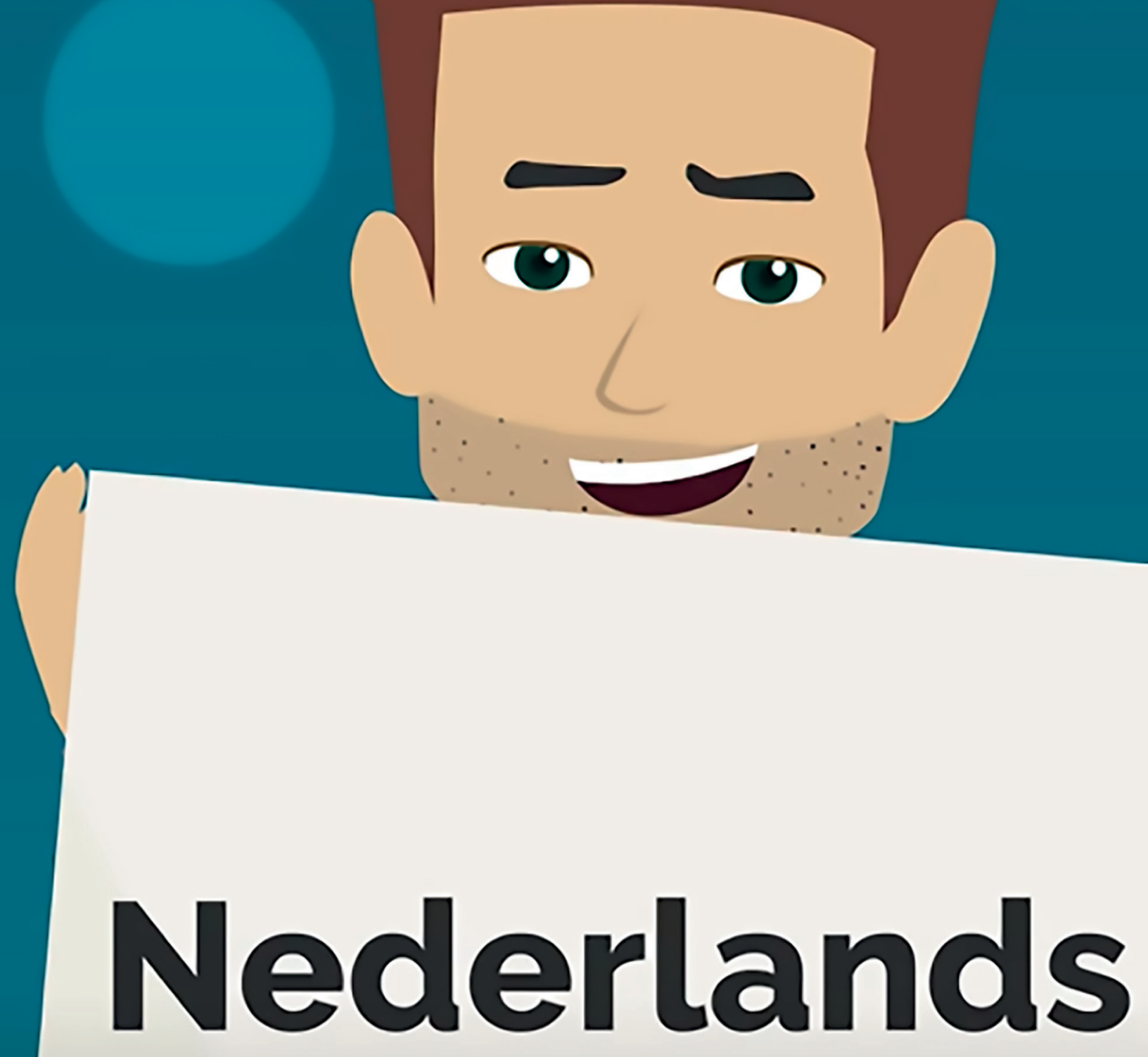


Samenvatting

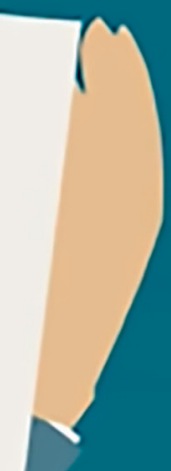




\section{Samenvatting}

Het belangrijkste doel van dit proefschrift is om de ontwikkeling en de effectiviteit van het eHealth-programma, Mijn Diabetes Profiel, te beschrijven. Het doel van het programma was om therapietrouw van type-2 diabetes patiënten te verbeteren aan kern behandelingsgedragingen, dat wil zeggen het verhogen van lichamelijke activiteit, het verbeteren van therapietrouw aan orale medicatie en insuline voorschriften, en het verlagen van de calorie-inname uit ongezonde snacks.

Hoofdstuk 1 bevat een algemene inleiding over diabetes mellitus, de gevolgen hiervan, managementstrategieën en het opvolgen van de behandeling. Diabetes Mellitus is een progressieve chronische ziekte die wordt gekenmerkt door hyperglykemie en het onvermogen van het lichaam om een optimale glucosestofwisseling te onderhouden. In 2045 zullen wereldwijd meer dan 600 miljoen mensen diabetes hebben, waarvan negen van de tien de type-2 diabetes variant. Behandelingsstrategieën bestaan uit het verhogen van de lichamelijke activiteit, het verbeteren van voedingspatronen en, indien van toepassing, het naleven van medische behandelingen, zoals het nemen van orale bloedsuikerverlagende medicatie en behandeling met insuline. Naleving door patiënten is een serieuze uitdaging. Therapieontrouw vindt plaats over verschillende behandelingsgedragingen heen en wordt gekoppeld aan aanzienlijke individuele, maatschappelijke en economische gevolgen. Het is daarom duidelijk dat therapietrouw moet worden verbeterd en nieuwe manieren moeten worden ontdekt waarmee patiënten hun behandeling beter kunnen naleven. De inleiding biedt verder een inzage in het theoretische kader dat is toegepast binnen dit proefschrift. Ook wordt er gesproken over computer-tailoring technologie als middel om ervoor te zorgen dat patiënten met diabetes hun behandeling beter naleven.

Hoofdstuk twee beschrijft een kwalitatief onderzoek dat heeft plaatsgevonden onder patiënten met diabetes type-2 en gezondheidsprofessionals die zijn betrokken bij het onder controle houden van diabetes. Het onderzoek richt zich op de omvang van therapieontrouw aan behandelingsgedragingen onder patiënten, belangrijke persoonlijke overtuigingen met betrekking tot het opvolgen van behandelingsgedragingen en of en hoe succesfactoren voor het opvolgen van de behandeling zijn toegepast in diabetesconsulten. De resultaten lieten zien dat het opvolgen van de behandeling met betrekking tot een gezonde levensstijl en medisch gedrag suboptimaal was. Het lijkt erop dat patiënten voor sommige behandelingsgedragingen meer kennis hebben en meer op de hoogte zijn van risico's dan voor andere gedragingen. Professionals en patiënten benoemden veel voordelen en geen nadelen van het naleven van gedrag dat hoort bij een gezonde levensstijl. Zowel voor- als nadelen werden gerapporteerd voor het naleven van medisch gedrag.

De eigen-effectiviteit van patiënten met betrekking tot het naleven van behandelingsgedrag leek lager te zijn in situaties waar routine werd doorbroken. Eenzelfde situaties werden 
geïdentificeerd voor afzonderlijke behandelingsgedragingen. Ten slotte, succesfactoren voor het opvolgen van behandeling werden onvoldoende toegepast in consulten. Gezondheidsprofessionals melden dat geringe, drukke en korte consulten de toepassing van dergelijke succesfactoren belemmerd kunnen hebben. Onze conclusie luidt dus dat de naleving van behandelingsgedragingen door patiënten suboptimaal is, niet-naleving plaatsvindt over verschillende vormen van gedrag, overtuigingen voor toepassing in interventies kenbaar zijn gemaakt, en nieuwe manieren onderzocht moeten worden om succesfactoren toe te passen.

Hoofdstuk drie beschrijft een longitudinaal onderzoek dat tot doel had psychologische voorspellende factoren van therapietrouw aan orale bloedsuikerverlagende medicatie te identificeren. Ook is binnen dit onderzoek een vergelijking gemaakt in therapietrouw prevalenties tussen twee zelf-rapportage instrumenten. In de eerste vragenlijst is therapietrouw in kaart gebracht middels de ProMAS en MARS-5 vragenlijsten, evenals sociaal-cognitieve variabelen en demografische factoren. Binnen de tweede vragenlijst is de ProMAS gebruikt als een uitkomstmaat en zijn de sociaal-cognitieve variabelen als gemeten in de eerste vragenlijst gebruikt om de therapietrouw te voorspellen middels een meervoudige lineaire regressieanalyse. Met de afkappunten van >15 voor de ProMAS en $>23$ voor de MARS-5, was respectievelijk 47\% en 90\% van deelnemers therapietrouw aan hun orale bloedsuikerverlagende medicatie. Gegevens van de ProMAS kwamen meer overeen met objectief verzamelde gegevens als men kijkt naar het percentage van patiënten dat als therapietrouw kan worden beschouwd. Terugkerende voorspellers van betere therapietrouw waren een laag opleidingsniveau, lagere ernstinschatting van risico's, en een hogere eigeneffectiviteit en bereidheid om de medicatie te gebruiken. Na correctie voor therapietrouw als gemeten in de eerste vragenlijst, bleven een laag opleidingsniveau en een hogere eigeneffectiviteit over als significante voorspellers. Kortom, de ProMAS lijkt beter om te kunnen gaan met valkuilen die aanwezig zijn in beschikbare zelf-rapportage instrumenten. Suboptimale therapietrouw benadrukt de behoefte aan effectieve interventies die therapietrouw kunnen bevorderen. Interventies moeten met name hoger opgeleide patiënten en patiënten met een lage eigen-effectiviteit in situaties die niet in de dagelijkse gang van zaken voorkomen, als doelgroep hebben.

Hoofdstuk vier beschrijft de ontwikkeling van het Mijn Diabetes Profiel programma en een protocol voor het uitvoeren van een gerandomiseerd gecontroleerde studie om de effectiviteit daarvan te onderzoeken. De resultaten van hoofdstukken twee en drie zijn aangevuld met kennis die is opgedaan uit eerder ontwikkelde programma's waarbij computertailoring technologie gebruikt wordt, evenals input die vergaard is vanuit een stuurgroep. Samen boden deze bronnen input voor de ontwikkeling van het eHealth-programma Mijn Diabetes Profiel. Als gevolg hiervan besteedt het programma aandacht aan therapieontrouw aan gezonde leefstijl gedragingen en medisch gedrag, en wordt het programma afgestemd op de wensen en behoeften van onze doelgroep en anderen die deel uit maakten van de 
commissie. Verder bevat het programma ook succesfactoren voor therapietrouw die naar voren zijn gekomen uit literatuuronderzoek. Bijvoorbeeld, het gebruik van een theoretische onderbouwing, interactieve inhoud en feedback die is afgestemd op relevante fases en determinanten van gedragsverandering, visuele ondersteuning en de mogelijkheid om therapietrouw te monitoren.

In hoofdstuk vijf wordt gesproken over de evaluatie die werd uitgevoerd inzake de werving van patiënten met diabetes type-2 door verpleegkundigen om deel te nemen aan ons gerandomiseerd gecontroleerde studie. Het doel van deze studie was om het aantal patiënten dat per verpleegkundige werd geworven in kaart te brengen, hoe dit totaal tot stand kwam tijdens de wervingsperiode, en om te onderzoeken welke factoren het uiteindelijke wervingstotaal van de verpleegkundigen hebben beïnvloed. Gegevens over het wervingstotaal en het verloop van het wervingssucces werden gehaald uit studiegegevens (NTR6840). Op basis van het gestandaardiseerde totale aantal geworven patiënten werden verpleegkundigen gecategoriseerd als verpleegkundigen zonder wervingsresultaat, verpleegkundigen met lage werving, verpleegkundigen met gemiddelde werving of verpleegkundigen met hoge werving. 96 verpleegkundigen namen deel aan onze studie en wierven gemiddeld zeven patiënten (spreiding 0-32), wat enigszins lager lag dan het minimumaantal van acht patiënten dat beoogd was. Vijfteen verpleegkundigen wierven geen enkele patiënt. De meeste patiënten werden geworven vlak nadat de wervingsperiode van start ging. Verpleegkundigen die geen patiënten wisten te werven vlak na aanvang van de werving bleken uiteindelijk geen tot weinig patiënten te werven. De gegevens laten een relatief hoog succes zien bij het begin van de werving dat op den duur geleidelijk minder wordt. Verpleegkundigen met een hoog wervingstotaal hadden over het algemeen meer succes tijdens de volledige wervingsperiode terwijl verpleegkundigen met een lage werving meestal (alleen) tijdens de eerste maanden. Telefooninterviews afgenomen onder een subset van verpleegkundigen per groep laten zien dat wervingsbarrières en facilitators zich voordeden op organisatorisch, studie- en patiëntgebied, maar voornamelijk bij kenmerken van de verpleegkundigen zelf. In tegenstelling tot verpleegkundigen zonder wervingssucces of verpleegkundigen met laag wervingsresultaat, lieten verpleegkundigen met een middel en hoog wervingsresultaat meer diepgaande kennis zien over de studie en de studievereisten. Ook lieten zij de persoonlijke voordelen voor deelname meer naar voren komen, spraken zij minder over belemmeringen en pasten zij meer wervingsactiviteiten, herinneringen en coping strategieën toe om het hoofd te bieden aan belemmeringen. De conclusie is dat vroegtijdig wervingssucces moet worden aangemoedigd en een persoonlijke aanpak verpleegkundigen kan helpen om succesvol te worden en te blijven.

In hoofdstuk zes zijn de resultaten weeggegeven van de gerandomiseerd gecontroleerde studie die werd uitgevoerd om de effectiviteit te onderzoeken van het Mijn Diabetes Profiel programma ten opzichte van een wachtlijst principe voor controle patiënten. De effecten van de interventie werden onderzocht op algehele therapietrouw aan 
behandelingsgedragingen en op afzonderlijke therapietrouw aan behandelingsgedragingen . Zestig procent van de patiënten die de eerste meting voltooiden werden behouden na de nameting, wat een belovend resultaat is voor eHealth-interventies. Algehele therapietrouw aan behandelingsgedragingen verbeterde aanzienlijk bij patiënten die werden toegewezen aan de interventie, wat weergegeven werd door een kleine tot gemiddelde effectgrootte $(d=.24)$. Wat betreft afzonderlijke therapietrouw aan behandelingsgedragingen konden wij een aanzienlijke verlaging zien van de calorie-inname uit ongezonde snacks $(d=.38)$. Kleine tot gemiddelde positieve interventie-effecten werden waargenomen voor therapietrouw aan orale bloedsuikerverlagende medicatie $\left(d={ }_{18}\right.$ ) en behandeling met insuline ( $d$ $=.28$ ). Deze effecten waren echter niet significant, wat mogelijk te maken heeft met een gebrek aan studie-power van deze medische gedragingen. Aangezien het Mijn Diabetes Profiel programma meerdere succesfactoren heeft toegepast voor eHealth-interventies die naar voren zijn gekomen uit literatuuronderzoek, zoals een theoretische onderbouwing, gepersonaliseerde content en een aanpak van meerdere behandelingsgedragingen, is het mogelijk dat deze bijgedragen hebben aan het succes van de interventie. eHealth heeft laten zien een effectieve manier te zijn om therapietrouw van type-2 diabetes patiënten te verbeteren. Toekomstig onderzoek is nodig om na te gaan hoe implementatie op brede schaal het beste vorm kan worden gegeven en of en hoe de integratie van eHealth in de algemene zorg van toevoegende waarde kan zijn.

Totslotwordtinhoofdstukzeveneenalgemenediscussieenconclusiebeschrevendiegebaseerd zijn op de studies die aan bod zijn gekomen in het proefschrift. Het Mijn Diabetes Profiel programma was effectief in het verbeteren van therapietrouw aan behandelingsgedragingen bij patiënten met diabetes type 2. Er zijn echter onderzoeksgebieden waarvoor verder onderzoek vereist is. Op dit moment zijn langer termijn gevolgen van het programma met betrekking tot verbeterde therapietrouw aan behandelingsgedragingen en gerelateerde secundaire uitkomstmaten onbekend. Bovendien moeten een kostenbatenanalyse en een procesevaluatie worden uitgevoerd en moet de optimale manier van levering voor implementatie op grote schaal worden onderzocht om het programma op de markt te brengen, het programma te finetunen en de impact ervan te vergroten. Ten slotte heeft de integratie van eHealth-interventies in algemene zorg, blendend care, laten zien gunstig te zijn, maar is er verder onderzoek nodig met betrekking tot het Mijn Diabetes Profiel programma. 


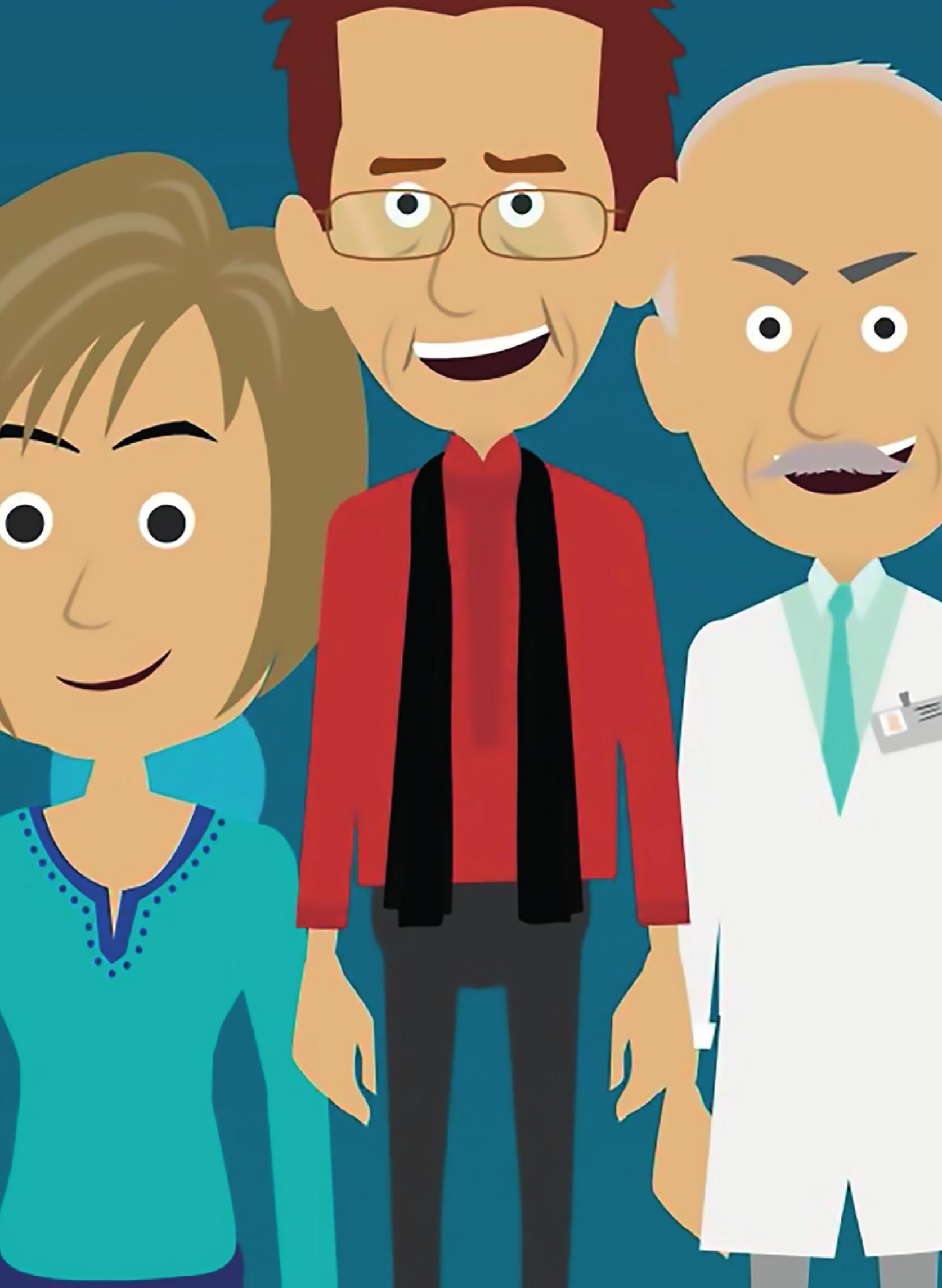


Dankwoord 


\section{Dankwoord}

Na ruim 4 jaar is mijn proefschrift af en is het tijd om een hele rits aan mensen te bedanken. En hoewel ik niet hou van clichés is het hier zeker op zijn plaats om te zeggen dat dit proefschrift zonder jullie niet tot stand was gekomen.

\section{Mijn promotieteam}

Hein, jij belde mij eind 2014 op om mij welkom aan boord te heten. Ik kende je natuurlijk als docent en hoogleraar van mijn tijd in de Health Education and Promotion master, uiteraard als fouding father van het alom bekende I-Change Model en als pionier op het gebied van computer-tailoring technologie. Twee dagen na mijn start viel je bij mij binnen en zei: "wil je mee naar Cyprus, dan doe maar even dit bestand analyseren, een abstract schrijven en dien hem maar in bij de EHPS." EHPS? Zo geschiede het. De toon was gezet: even dit, even dat, maar alles diende het doel van het project en het opbouwen van een netwerk. In overleggen probeerde je mij vooral bij de hoofdzaken te houden als ik weer eens honderdduizend ideeën had terwijl ik de 80 namen opschreef waarmee ik volgens jou contact moest leggen. Ook was het vaak een toets-uurtje of ik de theorie wel diepgaand genoeg bestuurd had. Dennis omschreef je, zoals je zo vaak zelf zegt, als de 'advocaat van de duivel' al zou rechterhand ook niet misstaan :-). Het is maar goed dat we enige 'darlings' aan studies gekilld hebben, anders was ik nu nog aan het schrijven of video's aan het maken in Vyond en dat zou het budget niet ten goede komen. Excuses dat mijn Word bestanden bij jou significant vaker crashten dan bij andere aio's en dat je vaak 8 keer opnieuw moest beginnen met feedback geven, uiteraard volgens het Molenaars principe. Soms vraag ik me af hoe je dat doet; 30 aio's, 10 masterstudenten, aanvragen schrijven, en heel vaak 'reloading batteries', zeilen, zingen, dansen, tennissen, en choreograferen. Chapeau. Dank voor het vertrouwen, en om in jouw stijl af te sluiten vriendelijke groeten en best wishes.

Nicolaas, ik zag je in 2014 wel eens rondlopen bij de Maastricht Studie en dacht: zo zien professoren er dus uit: lange witte jassen, een wijze snor en altijd druk. Niet wetende dat jij een tijd later mijn promotor zou worden. Ik ging op onderzoek uit bij mijn collega Julianne van der Berg die jou omschreef als een hartelijke en warme man, en achteraf kan ik het alleen maar met haar eens zijn. Jouw klinische toevoegingen binnen het project evenals jouw brede kennis op het gebied van diabetes en de zorg daaromheen waren zeer welkom. En hoewel je, volgens jezelf, vaak in een toestand van alzheimer-light verkeerde en ik weleens geduldig moest wachten op feedback, nam je op de geplande overlegmomenten altijd rustig de tijd om zaken te bespreken en uit te pluizen. Zo mocht ik ook af en toe bij jou thuis langskomen om voorstellen of vragenlijsten tot in detail door te spreken. Van het spaarzame uurtje dat er stond voor het overleg, werd in het eerste kwartier rustig een kopje koffie gezet, melk opgeschuimd en over andere dingen dan onderzoek gepraat. Je hebt me veel geleerd over diabetes, onderzoek doen, en de politiek en keuzes maken binnen een promotie-traject, en ik wil je hier graag voor bedanken. 
Ciska, als dagelijks begeleider hadden wij het meeste contact. Vaak was het een gestructureerd wekelijks overleg waarin ikeen stijgende lijn zag in jouw verwachtingen van mij als onderzoeker. Hoewel ik soms tot in de diepste diepte mijn keuzes moest beargumenteren, denk ik wel dat je op deze manier mij hebt gestimuleerd om het beste uit mezelf te halen. Zo merk ik dat ik in mijn conceptueel vermogen en schrijfstijl flinke stappen heb gemaakt en vooral niet de functionaliteit van dingen uit het oog probeer te verliezen. Ook is door jou mijn vocabulaire aan (spreek)woorden flink uitgebreid. Naast de Engelstalige wetenschappelijke termen, viel er altijd wel een (spreek)woord te ontleden als 'bot vangen','het moet wel hout snijden' of 'boude uitspraak' (gewaagd). Ik heb veelal het idee dat wij samen het project gedragen hebben; tot in de diepte dingen voorbereiden, of het nu artikelen of interventiecomponenten waren, en deze ter goed- (of af-) keuring voorleggen aan de rest van het team. Dankjewel voor je uitdagende begeleiding en fijne samenwerking.

\section{Deelnemers aan studies}

Zonder deelnemers geen studies. De vele mensen en zorgverleners (praktijkondersteuners, diabetesverpleegkundigen, artsen, diëtisten en eHealth experts) wil ik dan ook bedanken voor hun bereidheid mee te doen met de vele studies die we verricht hebben. Het werven van voldoende deelnemers blijft altijd een lastig punt in het doen van onderzoek. Ik vond het dan ook fijn en interessant om een van mijn studies hieraan gewijd te hebben. In het bijzonder wil ik dhr. van Rooijen bedanken voor zijn nauwe betrokkenheid bij de studies, het vormgeven en pilot testen van mijn interventie, en het verzorgen van een patiëntendemonstratie bij mijn colleges.

\section{Verpleegkundigen MUMC+}

De verpleegkundigen van de diabetespoli MUMC+ wil ik bedanken voor hun gastvrijheid, vriendelijkheid en bereidheid te willen helpen bij welke studie dan ook. In het bijzonder wil ik Yvonne Koolen en Jean-Gilles Ruyters bedanken. Yvonne, je was vanaf het begin betrokken bij het opzetten van mijn studies en interventie, evenals bij DIEP. Ik denk dat je erg trost mag zijn op de manier hoe je die rol hebt ingevuld. Daarnaast ben je ook nog eens een heel fijn persoon om mee te praten en samen te werken, én ben je niet te beroerd om ook je duidelijke mening te uiten als je iets vanuit verpleegkundig perspectief gevraagd werd. Dankjewel, en moge je nog vele mooie jaren bij het MUMC en op de fiets hebben.

\section{De leescommissie}

Experts op het gebied van computer-tailoring, eHealth, gezondheidspsychologie, diabetes en therapietrouw die jouw stuk gaan lezen en beoordelen. Dank, Nanne, Stef, Hilde, Catherine en Trudy voor jullie oordeel en tijd die jullie in het lezen van mijn proefschrift hebben gestoken. Nanne, je hebt mij ooit tijdens een kerstlunch overtuigd dat het écht mogelijk is dat een witte wijn lekkerder kan zijn dan een rode, maar dan moest het wel een hele goede witte zijn. Ik wacht in alle rust je advies af. 


\section{Stuurgroep}

Vanaf het begin van het project hebben wij een stuurgroep opgericht met daarin afvaardiging vanuit eHealth, de doelgroep, onderzoek, diëtetiek, eerste- en tweedelijnszorg, en praktijkgericht onderzoek. Het op deze manier co-creëren van onze interventie is zeker van meerwaarde geweest en heeft ons vanuit verschillende invalshoeken de interventie laten aanvliegen en opbouwen. Naast mijn team wil ik daarom Silvia, Sonja, Anna, Yvonne, Erwin, Ronald, Esther, Rik, Dirk, en Pie bedanken.

\section{Het secretariaat}

Kim, Patricia, Denise, Daisy, Helga, Joyce en Yvonne, dank voor jullie ondersteuning in de afgelopen jaren. Patricia en Kim, mijn excuses dat ik vaak niet tijdig mijn afwas deed en daardoor kopjes, bordjes, bestek etc. veel te laat terugbracht naar de vergaderkamer, dat ik vaak mijn sleutel kwijt was en er weer een nieuwe besteld moest worden, of dat mijn auto weer eens verkeerd geparkeerd stond en er een boze mail naar GVO volgde. Het ga jullie goed, en moge de schilderijen nog lang in de gang hangen.

\section{Ouders}

Dank voor jullie steun in de afgelopen jaren, hopelijk begrijpen jullie na het lekenpraatje eindelijk wat ik nou echt voor onderzoek doe, want dat is en blijft toch altijd lastig om uit te leggen. Ik ben blij dat het nu zo gaat zoals het gaat met jullie en laat dat niet meer los.

\section{Oma}

Ja, waarom niet. Helaas moesten we gedurende mijn promotietraject afscheid van je nemen. Ik denk echter, en jij zelf ook, dat veel mensen zouden tekenen voor de ruim 96 jaar die je hebt mogen worden. Ik heb nog ooit nagedacht om je aan te stellen als mijn paranimf, dan had je daar met 98 misschien nog een stelling mogen voorlezen, maar jammer genoeg is ons dat niet gegeven. Dankjewel voor de wijze levenslessen die je me hebt meegegeven, en ik weet zeker dat je op de dag van mijn verdediging een oogje in het zeil houdt.

\section{Assistentie}

Door de jaren heen heb ik heel wat assistentie gehad bij mijn projecten, zowel van medewerkers als student-assistenten. Jullie zijn van onschatbare waarde geweest voor mijn project en graag wil ik jullie dan ook bedanken voor jullie werk en toewijding.

Claire, dank voor je fijne samenwerking. Jij was de expert op het gebied van tailoring en ik ben heel blij dat jij mij daarin geassisteerd hebt. Ook deelden we de frustraties als het opeens toch weer allemaal anders moest, maar gelukkig hebben we dat overleefd.

Kenny, jij was in het begin bij mijn project betrokken, eveneens bij het vormgeven van mijn programma en het uitwerken van interviews. De nauwkeurigheid en rust waarmee jij te werk 
ging is bewonderenswaardig te noemen. Ik hoop dat je nu je plekje hebt gevonden waar je collega's ook mogen genieten van deze capaciteiten.

Merel en Marion, jullie waren eventjes betrokken bij mijn project maar daarom niet het noemen minder waard. Ik ben blij voor jullie dat jullie een eigen PhD-project hebben binnengesleept en ik weet zeker dat jullie dit met succes gaan afronden.

Raesita, sinds de middelbare school mijn klasgenoot en opeens werkten we samen op hetzelfde project. Beiden oes Kirchroa, en dan samenwerken in Maastricht, hoe is het zo ver gekomen. Je bent écht oprecht een heel fijne collega en je levert kwalitatief heel goed werk. Dat zijn mijn collega phd-studenten waarvoor je gewerkt hebt, denk ik volledig met mij eens. Niet alleen de snelheid waarmee jij tailor-builder hebt geleerd, maar ook de manier hoe je dingen kunt uitleggen en werk verzet, tekenen volgens mij de persoon die je bent. Ik vind het nog steeds bijzonder dat ze mensen als jij niet langer vastleggen, maar helaas ga ik daar niet over. Ik wens je veel plezier bij de Maastricht Studie en we komen elkaar vast nog wel tegen.

Yil, toen ik in mijn eerste jaar van mijn promotietraject lesgaf, vroeg ik de toenmalige eerstejaars wie er bij mijn project wilde helpen. Zo kwam je bij mijn project terecht en ging vrijwillig aan de slag met interviews uitwerken (erwtjes) en data-invoer. Niet het leukste werk om vrijwillig te doen, maar je klaagde nooit, gapen wel overigens. In de loop van de jaren bleef je bij mijn project betrokken en hoewel ik mijn best deed om vrijwillige assistentie betaald te laten worden, hield de projectbaas de vinger meestal op de knip. Nu heb je je eigen PhD project, gaapt nog veel meer dan eerst, maar staat wel altijd klaar voor een lunch of wandeling. Je bent een heel fijne en optimistische collega en dat siert je. Dank voor al je vrijwillige uurtjes in het MDP project en je gezelligheid en succes met het vervolg van je eigen project.

Lieve, eigenlijk hoor je niet in het rijtje assistentie of collega's thuis, maar ik heb je toch maar ergens bijgezet. Je interesse om bij een project betrokken te raken eindigde in een artikel dat we grotendeels samen geschreven hebben, en waar we menig uur of dagdeel samen aan gewerkt hebben. Niet alleen leverde dit een mooi artikel op, maar ook fijne gesprekken. Ik denk dat je trots op jezelf mag zijn met het werk dat je geleverd hebt, dat heb je toch maar even gedaan als $2^{\mathrm{e}} / 3^{\mathrm{e}}$ jaars student. Ik denk dan ook dat een PhD project goed bij je zou passen, maar dat moet je natuurlijk maar helemaal zelf uitvogelen of je dat wil. Stiekem merk ik dat je er meer en meer open voor gaat staan en ik zou je bij iedereen zonder twijfel aanbevelen. In ieder geval veel succes bij het afronden van je master en alles wat daarna op je pad komt.

\section{Paranimfen}

Carolin, a.k.a. dr. Muschalik, wat ben jij een fijne collega en fijn mens. Auch an dir herzlichen glückwunsch zu deiner promovierung. Vanaf de eerste tot de laatste dag zaten we op dezelfde plek, aan elkaars overkant van de gang. En als de deuren open waren dan was één blik of 
e-mail vaak genoeg om te begrijpen wat we bedoelden. Er bestond dan ook geen twijfel dat ik jou wilde vragen om mijn paranimf te zijn. Ik bewonder je rust, relativeringsvermogen, mindfulness en de manier waarop je 45 minuten kunt doen over het eten van een pizzapunt. We hebben heel veel gelachen om de meest onzinnige dingen of uitspraken, en ik kon het niet laten om ze nog een keer hier te benoemen: Beheeeeeevior, appreciaaaation, kijk eens kijk twee, wat is de STAVAZA, to know is to know that you know nothing, IKEA: international kidney essembly associeeeeetion, @Zuyderland, working elsewhere, the day the duck got saved, you knoooow, beheeevior change wheeeel, kaput day, HALT STOP, chai-latte, wat zijn jullie aan het doeeeen, awaiting reeeeviewer selection, coping plants, self-EFFcacy, When I want to smoke, I make a plan not to smoke, and then I will not smoke, Prögram, gehirm amputiert, jullie hebben mij 10000 euro gekost, greetings of the day, 'dear prof. dr. Muschalik, we are pleased to inform you we have a special issue on cheese consumption adherence in Korean nurses, and would like you to contribute. You have time till tomorrow, adHIERENCE, WAAR IS DE PRULLENBAK?!, the journal of no impact, pills give me chills, I won't travel for a poster, affenkopf, fameeeeuze, genereeeuze, theetje Stanislas? Ik ga deze momenten echt heel erg missen. Thanks for all your support and great collegiality.

Guido, ouwe Madlipz koninig. Niet alleen ben je een goeie kerel, maar ook in veel opzichten hetzelfde als ik. Zo hou je ook graag van een feestje, dingen organiseren, weer eens een veadige vriendenweekend of Finland trip, een vuurtje stoken, frustratie als de rest van de vrienden niet hetzelfde enthousiasme hebben als wij, en uiteraard het maken van Madlipz filmpjes of satire nieuws. Eigenlijk zou je misschien zelf eens moeten denken aan een PhD project: een uitgebreide literatuurstudie naar de opwarming van de aarde heb jij immers al gedaan. Bedankt dat je mijn paranimf wil zijn en laten we samen met de rest van de vriendengroep nog veel mooie feesten mogen beleven.

\section{Vrienden en collega's}

De Veadige - East Holz Massive en TCK'54 vrienden, bedankt voor jullie steun en toeverlaat. De vele feesten, Ardenne weekenden en Finland trips waren een welkome afwisseling in de afgelopen jaren.

Kai, ik zou mijn boekje zonder enige twijfel zo inleveren om je weer erbij te hebben vriend. In mei 2017 hielp je me nog met het vouwen van enveloppen voor mijn studie en maakten we de nodige filmpjes voor onze Facebook pagina. Het is niet te bevatten dat je er een paar maanden later niet meer was. Het is een groot gemis voor iedereen en onze vriendengroep. En hoewel het er niet minder erg door wordt, hoop ik, en weet ik eigenlijk zeker, dat jij je rust gevonden hebt.

Esther en Karlijn, onlosmakelijk van elkaar verbonden zijn jullie twee. Bedankt voor de gezellige momenten en voor het eten dat ik altijd bij jullie kwam stelen (ondanks alle vliegen en muizen) als mijn eigen eten weer eens op was. Ook jullie gaan zonder twijfel je project 
goed afronden. Het bord met achievements wordt voller en voller. Veel succes met jullie bedrijf dat jullie gaan starten daarna waar ik nog steeds ENORM benieuwd naar ben, want als ik jullie mag geloven zal het geld binnenstromen. Moge jullie lach, en met name die van Esther, nog zolang jullie projecten duren, luid klinken op de GVO gangen.

Marika, het laatste half jaar was jij mijn kamergenoot en dat was een gezellige tijd. Heden, bliksem, Frjentsjer. Enkele van de Friese woorden die je me geleerd hebt. Dat taaltje kun je beter niet al te lang aanhoren, maar goed, dat zal wel wederzijds zijn voor het Kerkraads dialect. Hopelijk vind je genoeg deelnemers voor je studie en krijg je een ooit een mooi PhD project. En als dat (voorlopig) niet lukt, kun je nog altijd terug naar je favo-job bij de Mac.

Dennis en Latifa, ik wil jullie bedanken voor jullie fijne collegialiteit en hulp als ik vragen had over mijn project of bepaalde studies. Als twee PhD'ers die 'kort' voor mij begonnen waren, waren jullie voor mij in de ideale positie om mij op weg te helpen. Dank voor de tijd die jullie daarvoor hebben genomen en veel succes verder bij jullie wetenschappelijke carrières.

Niki, we leerden elkaar kennen bij de Maastricht Studie in 2013 en niet lang nadat ik naar GVO ging voor mijn promotieonderzoek volgde jij datzelfde pad. Hoe gezellig was het dat jij bij mij op de kamer kwam: 'Ja maar JOS? Doe mij eens 5 euro's geven voor Toro Picine', of het schrijven van het GVO-ALL NEWS. Ik denk dat je je plekje zometeen wel gevonden hebt in het leefstijl en coaching gebeuren. Misschien kom je nog wel ooit bij je droombaan uit bij de Efteling, het land van ooit, of bij onze vriend Chiel van Adaptics. Ik blijf je gewoon taggen op Linkedln bij hun vacatures totdat ze je smeken om naar Nijmegen terug te verhuizen om directeur van SGM te worden. Hoewel ik ook denk dat je zeker je PhD project succesvol afgerond had. Dank je voor de leuke momenten en leuke collega die je was bij DMS en GVO. De kurk met handtekeningen van eind 2014 zal ik zoals beloofd meenemen op de dag van mijn verdediging.

Anke, je werkt nu ook al heel wat jaartjes bij GVO en hebt mijn traject ook redelijk vanaf het begin meegemaakt. Zo weet je als geen ander de momenten waarop het goed en minder goed ging en stond je altijd klaar om daarnaar te luisteren, over te praten/beppen, of je advies over te geven. Ik kan je daar niet genoeg voor bedanken. Ook denk ik aan de vele mooie en gezellige momenten. De Wednesday afternoon challenge, de plantenasiel battles, de tien kilometer, het organiseren van wie is de mol en de serieuze leedvermaak-blik waarmee je de kandidaten trakteerde op een rood scherm... Verder ben je gewoon een topper, een goed mens, een echte sfeermaker voor de afdeling, en wissel je de Brabantse taal af met native Engels alsof je er geboren bent. Het is dan ook een kwestie van tijd voor je je eigen PhD project binnensleept en nog jarenlang jokers verstopt op onvindbare plekken. Ik vind het heel jammer dat je er niet bij bent, maar ik weet zeker dat je aan de andere kant van de wereld geniet van je welverdiende vakantie. 
Steffi, we zijn ongeveer tegelijk begonnen maar je bent al een tijdje klaar met je onderzoek. Das hast du gut gemacht. Ik wil je bedanken voor de gezellige momenten en onze vele bezoekjes, met name in het begin, aan Banditos en wens je al het beste toe.

Dirk-Jan, ongelooflijk dat je nog voor mij promoveert, maar alleen maar iets om trots op te zijn. Gelukkig kan ik mezelf tot twee dagen van tevoren voorhouden dat er nog altijd iemand vóór mij is. Congratz, alvast, en veel succes bij het vervolg van je carrière.

Rik, dankjewel voor de fijne collegialiteit en samenwerking. Je bent denk ik een voorbeeld voor velen, niet alleen in het werk dat je verricht, maar ook op sociaal gebied. Je hebt altijd tijd voor een praatje, tijd om je advies te geven over een stuk tekst of idee, of tijd om te informeren hoe het gaat. Als ik zelf al vergeten was dat ik 3 weken geleden een presentatie had gegeven, dan vroeg jij nog hoe het was gegaan. Hopelijk kunnen we in de toekomst voor het een of ander nog samenwerken.

Caroline, ik bewonder je doorzettingsvermogen, harde werken en zorgzaamheid. Ik wil je ontzettend bedanken voor je support, met name in het laatste deel van het traject. Op naar meer rust, en tijd voor dingen die er echt toe doen!

ledereen die niet genoemd is of die ikvergeten ben te noemen, ook aan jullie een welgemeend dankjewel! 


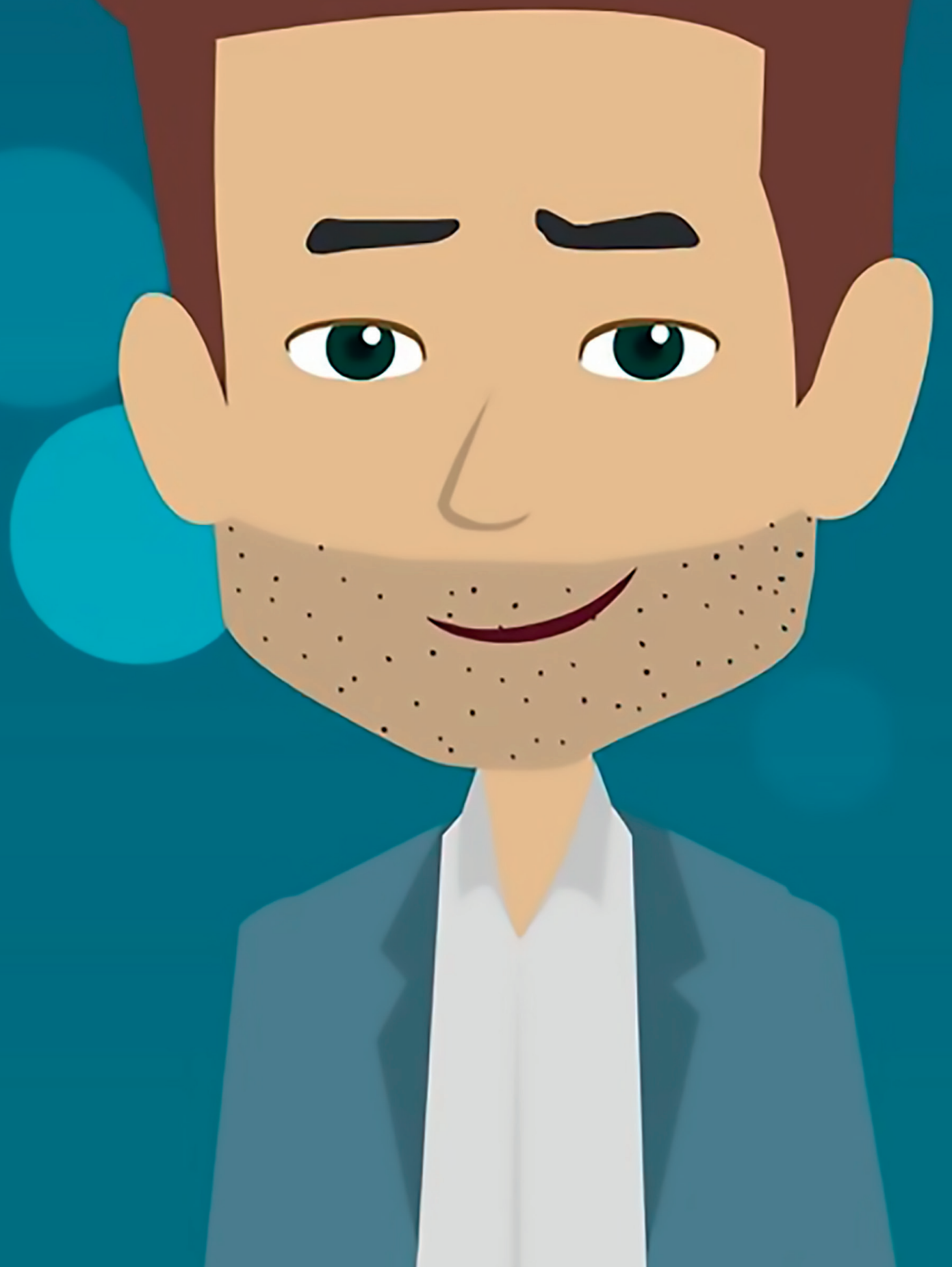




\section{About the author}




\section{About the author}

Stan Vluggen was born on June 29th 1989 in Kerkrade, the Netherlands. After completing secondary school (VWO) at College Rolduc in Kerkrade in 2007, he started studying Health Sciences at the Maastricht University. After one year, he moved to Nijmegen to study Sports, Health and Management at the University Of Applied Sciences Of Nijmegen. After choosing the health management direction in the second study year and obtaining the Bachelor of Health degree in 2012, Stan started the master's program Health Education and Promotion at the Maastricht University in the same year. One year later he graduated.

After obtaining the master's degree, Stan worked as an assistant on several small research projects for the Regional Health Authority of South-Limburg and the Care and Public Health Research Institute (CAPHRI). In November 2013, he started working as a research assistant for the Maastricht Study, a large prospective cohort study on the etiology of type 2 diabetes mellitus. During this period, Stan further developed his interest in conducting research as he wanted to get more insight in the whole process of conducting research rather than only data-collection, respectively.

In February 2015, Stan started working as a PhD candidate at the department of Health Promotion of the Maastricht University and was supervised by dr. Ciska Hoving, prof. dr. Hein de Vries, and prof. dr. Nicolaas Schaper. During four and a half years, he worked at several studies that are presented throughout this dissertation, combined his research with various teaching responsibilities, and obtained his University Teaching Qualification in 2019. Moreover, Stan was appointed as CAPHRI PhD representative for a period of almost two years. In 2019, Stan was nominated for an education award for best tutor of the Faculty of Health Medicine and Life Sciences within the Health Domain. He presented his research work at several national and international conferences. Currently, Stan remains being involved in research and teaching as he is appointed as postdoctoral researcher at the department of Health Services Research of the Maastricht University. 


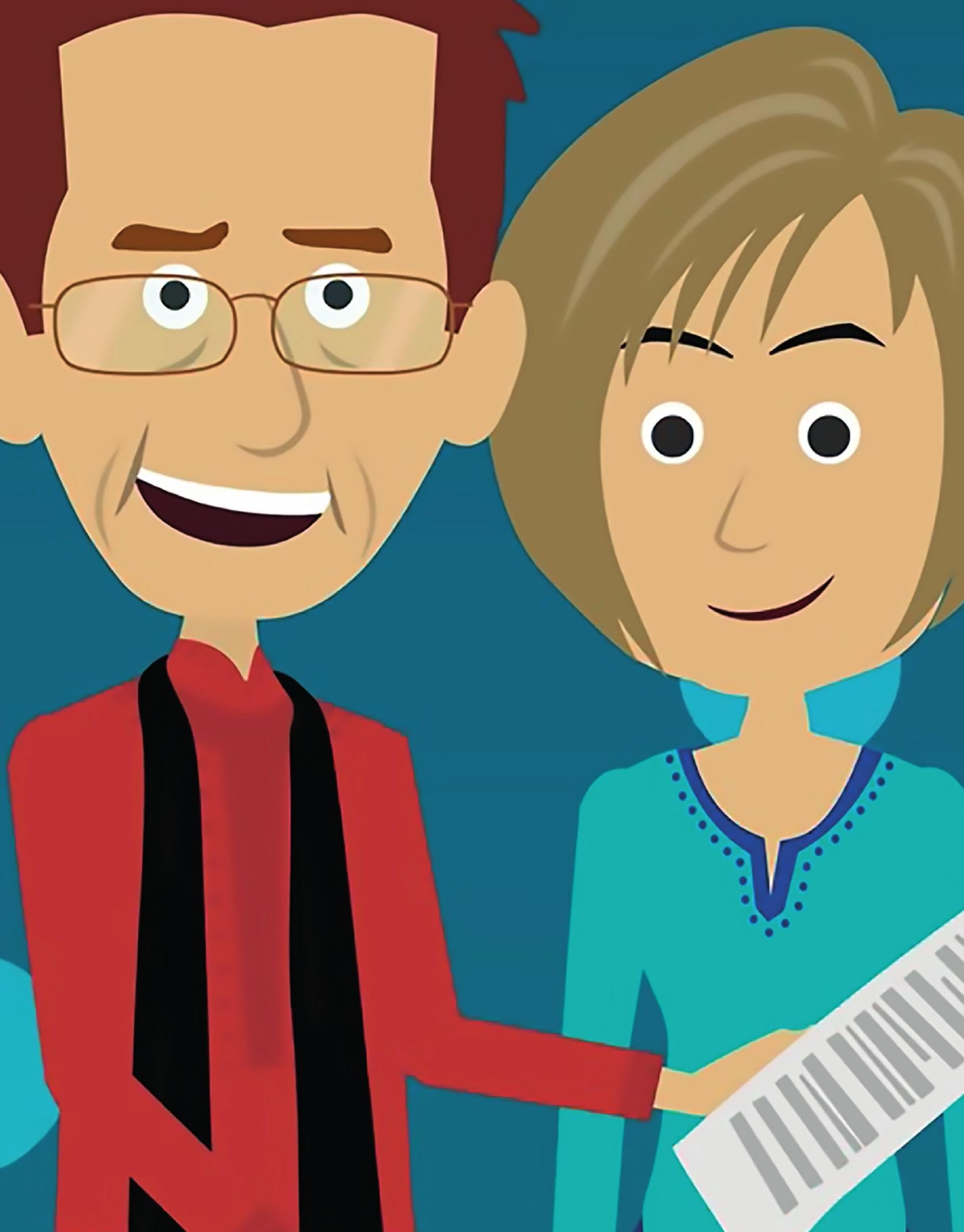


List of

publications 


\section{Publications presented in this dissertation}

Vluggen, S., Hoving, C., Schaper, N. C., \& de Vries, H. (2018). Exploring beliefs on diabetes treatment adherence among Dutch type 2 diabetes patients and healthcare providers. Patient Education and Counseling, 101(1), 92-98. https://doi.org/10.1016/j.pec.2017.07.009

Vluggen, S., Hoving, C., Schaper, N. C., \& de Vries, H. (2018). A web-based program to improve treatment adherence in patients with type 2 diabetes: Development and study protocol. Contemporary Clinical Trials, 74, 38-45. https://doi.org/10.1016/j.cct.2018.10.002

Vluggen, S., Hoving, C., Schaper, N. C., \& de Vries, H. Psychological predictors of Adherence to Oral Hypoglycemic Agents: an application of the ProMAS questionnaire. Epub ahead of print. https://doi.org/10.1080/08870446.2019.1672873

Vluggen, S., Hoving, C., Vonken, L., Schaper, N. C., \& de Vries, H. Exploring factors influencing recruitment success of nurses recruiting diabetes patients for a randomized controlled trial. submitted; under review

Vluggen, S., Candel, M., Hoving, C., Schaper, N., de Vries, H. The effectiveness of a web-based computer-tailored program to improve treatment adherence in patients with type 2 diabetes: randomized controlled trial. Submitted 


\section{Other publications}

Van het Schip, C., Cheung, K., Vluggen, S., Hoving, C., Schaper, N., de Vries, H. Development and Pretesting of Spoken Animated Self-Management video messages aimed to improve Physical Activity in People with Diabetes type 2. Submitted

Theunissen, K. A. M., Bos, A. E., Hoebe, C. J., Kok, G., Vluggen, S., Crutzen, R., \& Dukers-Muijrers, $\mathrm{N}$. H. (2015). Chlamydia trachomatis testing among young people: what is the role of stigma? BMC public health, 15(1), 651. https://doi.org/10.1186/s12889-015-2020-y

Vluggen, S., Hoving, C., Schaper, N. C., \& de Vries, H. De beweegrichtlijnen 2017: Hoe toepasbaar zijn de nieuwe Beweegrichtlijnen? To be submitted 
